\title{
Pulsed power applications for agriculture and food processing
}

\author{
Koichi Takaki,2 D $\cdot$ Katsuyuki Takahashi ${ }^{1,2} \cdot$ Nobuya Hayashi $^{3} \cdot$ Douyan Wang $^{4}$. \\ Takayuki Ohshima ${ }^{5}$
}

Received: 10 August 2021 / Accepted: 14 November 2021 / Published online: 4 December 2021

(c) The Author(s) 2021

\section{Abstract}

Recently, pulsed power technologies, including pulsed electric fields (PEFs) and time-modulated plasmas, are starting to be applied actively in agriculture and food processing. In the applications, compact pulsed power generators with moderate peak power and repetitive operation are developed for controlling discharge plasmas and electric field distribution. These applications are mainly based on the biological effects of a spatially distributed electric field and the chemically active species in the plasma. The PEFs are caused by applying pulse voltage between the electrodes and contribute to form pores on the cell membrane or to change conformation of protein. When the applied voltage exceeds the discharge onset criterion, plasmas are generated through the avalanche process of electron accelerated with intense electric field in a gas or liquids medium. The plasmas produce chemically active species, UV radiation, an intense electric field in the vicinity of discharge channel and shock waves, which also have different biological effects. The agricultural applications of pulsed power can be categorized as two phases: pre-harvest and post-harvest phases. The pre-harvest phase consists of seed germination, seedling growth, plant growth and growth mode change from vegetative to reproductive. Pulsed power technologies are used to promotion of seed germination, plant growth enhancement through direct stimulation or indirect effect such as inactivation of bacteria in soil and liquid hydroponic media, and promotion of mushroom fruit body formation. The postharvest phase consists of harvesting the agricultural produce, storing the products, transporting the products to consumers and food processing such as drying, pasteurization (sterilization of bacteria), permeabilization and fermentation. The pulsed power technologies are also used to keep freshness of agricultural produce through decontaminating airborne, inactivating bacteria and decomposition of plant hormone in the storage containers. The poration of cell membrane by PEF contributes improvement of extraction of juice, nutritional agents, and antioxidant metabolites such as polyphenols from agricultural products. In this review, at first, a basis of pulsed power system for agricultural applications and bio-effect by high-electric field exposure is outlined. After that, pre-harvest and post-harvest agricultural 
applications are described. The utilization of pulsed power technologies to contribute efficient food processing and improve food safety and quality is also described.

Keywords Pulsed power $\cdot$ Plasma $\cdot$ Agriculture $\cdot$ Growth $\cdot$ Freshness $\cdot$ Food processing $\cdot$ Protein conformation $\cdot$ Pulsed electric field $\cdot$ Electroporation

\section{Introduction}

Food supply chain from farm to consumers is an important topic to contribute a sustainable society. The agricultural and food industries have to respond to the demands of a growing population, both in terms of nutrition and consumer tastes such as antiaging and healthy food. These tasks have to be achieved within available resources and regulatory requirements related to the food safety and environmental protection. There are many technologies used in food production (agriculture, stockbreeding and fishery) and processing to achieve an effective food supply chain; however, novel and innovative technologies are required for emerging challenges of global food security including safety and quality issue in the modern food supply chain. The agricultural and food processing applications of pulsed power technologies such as intense pulsed electric field and time-modulated discharge plasmas have been studied and growing rapidly in researchers worldwide (Takaki et al. 2019). The repetitive operation compact pulsed power generators with moderate peak power for suitable in the agricultural and food processing applications have been developed by many researchers for temporal and spatial control of intense electric fields and discharge plasmas. The compact pulsed power generator suitable for the applications are important because the applications are mainly based on biological effects of the electric field (Akiyama and Heller 2017) and biochemical reactions by chemically active species in plasma (Graves 2012).

Pulsed power is the technology of accumulating energy during a relative long period of time and of releasing the accumulated energy in extremely short period as high-power pulse composed of high voltage and large current, however, moderate low energy, i.e., low time-averaged power (Akiyama and Heller 2017). When the pulsed high voltage is applied between arbitrary shaped electrodes, the intense pulsed electric fields (PEF) are produced between the electrodes, which causes biological effects such as electroporation (formation of pores on the cell membranes) of cell membrane (Zimmermann 1982), and conformational change in proteins (Guionet et al. 2021). These phenomena can be used in the agricultural applications such as inactivation of pathogenic bacteria, enzyme activity change, and extraction of nutritional agents and antioxidant metabolites from agricultural products (Takaki et al. 2019). When the high voltage is applied between electrodes over the discharge onset criterion, the discharge plasmas are generated, which causes biological effects through biochemical reactions by reactive oxygen and nitrogen species (ROS, RNS) supplied by the plasmas (Weltmann et al. 2010). The discharge plasmas also induce UV radiation, an intense electric field in the vicinity of discharge channel and shock waves, which also have different biological effects. 
The agricultural applications of pulsed power can be categorized as two phases: pre-harvest and post-harvest phases as shown in Fig. 1. The pre-harvest phase consists of seed germination, seedling growth, plant growth and growth mode change from vegetative to reproductive. Pulsed power technologies are used to promotion of seed germination (Eing et al. 2009; Attri et al.2021), plant growth enhancement through direct stimulation (Wang 2021) or indirect effect such as inactivation of bacteria in soil and liquid hydroponic media (Okumura et al. 2016; Takahata et al. 2015), and promotion of mushroom fruit body formation (Takaki et al. 2014). The post-harvest phase consists of harvesting the agricultural produce, storing the products, transporting the products to consumers and food processing such as drying, pasteurization (sterilization of bacteria), permeabilization and fermentation. The pulsed power technologies are also used to keep a freshness of agricultural produce through decontaminating airborne (Koide et al. 2013), inactivating bacteria (Ito et al. 2021), and decomposition of plant hormone in the storage containers (Takaki et al. 2020). The poration of cell membrane by PEF contributes improvement of extraction of juice, nutritional agents, and antioxidant metabolites such as polyphenols from agricultural products (Yang et al. 2016). In the process of fermentation, a metabolic process of yeast and bacteria activity is controlled by exposure with the intense PFEs (Ohshima et al. 2007, 2021).

For pulsed power generators in the agricultural applications, it is important to design as repetitive high-voltage output with optimum amplitude of voltage and waveform shapes, to deliver the moderate pulsed power into the biologic loads (Akiyama and Heller 2017). This energy flow can be based on simple circuits consisting of passive discrete resistive-inductive-capacitive elements, transformers (in lumped constant circuit) or transmission lines (in distributed constant circuit), and switches, which transfers the energy stored in the electric fields of capacitors or magnetic fields of coils (Bluhm 2006; Lehr and Rpn 2017). In this review, at first, a history of high-voltage application to the agriculture is described briefly in Sect. 2. Following Sect. 2, pulsed power generations and electric field distribution generated by applying high voltage between arbitrary shaped electrodes are outlined in Sect. 3.

The history of agriculture identified by crop production is older than civilization. The process of in agriculture is almost unchanged as selecting the plant for cultivation, preservation of the seed of crop plants, modifying the bed soil suitable for crop plant cultivation, seed planting, weeds elimination, protection of crop plants from enemies such as pathogenic bacteria and plant pests, and harvesting the agricultural products (Martin and Leonanrd 1967). These processes are categorized to the pre-harvest phase. The basis of phenomena in intense electric field for biological effects used in the agricultural applications are described briefly in Sect. 4. The

Fig. 1 Application in pre- and postharvest (Attri et al. 2021)

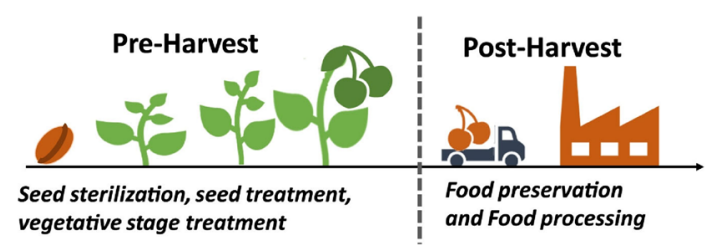


intense electric field accelerates charged particles by the Coulomb force, following that, an electron avalanches are formed and are developed in direction of the electric field. The electron avalanches cause an electrical breakdown (or discharge onset) between the electrodes when the electric field has enough amplitude to cause the electrical breakdown (Kuffel et al. 2000). After that, the agricultural applications of pulsed power in the pre-harvest phase are reviewed in Sect. 5. The promotion of seed germination, seedling growth enhancement, inactivation of pathogenic bacteria, and promotion of mushroom fruit body formation are described as the preharvest applications.

The post-harvest phase consists of harvesting the agricultural products, storing the products, transporting the products to consumers and food processing such as drying, pasteurization (sterilization of bacteria), permeabilization and fermentation. The pulsed power technologies including strong electric field and plasma can contribute for keeping freshness through decontaminating airborne, inactivating bacteria and decomposition of plant hormone in the storage containers. These applications are reviewed in Sect. 6. The poration of cell membranes by PEF contributes improvement of extraction of nutritional agents and antioxidant metabolites from agricultural products. These applications are described in Sect. 7. Finally, all applications are summarized in Sect. 8.

\section{History of electric field exposure for plant growth promotion}

Since the discovery of atmospheric electricity (electrostatic phenomena), many researchers had been interested in the relationship between the electrostatic phenomena and biological activities of living organisms i.e., plants and animals (Lemström1904; Barman and Bhattacharya 2016). In nature, the atmosphere and the ionosphere of Earth surface consists of the global atmosphere electrical circuit which produces the electrical field of a few-ten $\mathrm{V} / \mathrm{m}$ as natural atmosphere of plant growth and animal living fields. The electromagnetic wave of visible wavelength range (from 300 to $800 \mathrm{~nm}$ ) is important for plant growth because the visible electromagnetic wave (e.g., sunlight) is used in photosynthesis. The visible range electromagnetic wave also works as stimuli for germination of plant seed, blooming, and fruition. Then, sap flow of nutrient in the plant body is carried out through the rootlets, center, in the stalks to the proper sites controlled by electrical signals such as bioelectrical potential and ions. Therefore, all living things on earth have been living in the nature electric field and electrostatic phenomena in a long time from generation of life, i.e., around 4 billion years ago.

Lemström published the book of title "Electricity in agriculture and horticulture" in 1904 (Lemström 1904). The book describes that the plant growth and the yieldings of crop are improved by electrical treatment such as electric field and ions generated by applying high voltage, those effects are express by the term "electroculture" since the nineteenth century. The electrical treatment in crop production is not only direct treatment of plants but also cultivation bed soil by flowing the electric current (Barman and Bhattacharya 2016). The growth enhancement of plants by applying high voltage which induces generation and flowing ions in ambient air 
was first confirmed by Maimbray in 1746 (Solly 1845). Followed by the work by Maimbray, electrical treatment was used to improve yielding of crop production by Berthelon in 1870. Basty and Sanborn got positive results on plant yield by applying modified atmospheric electricity, whereas Solly found no appreciable change (Basty 1908; Sanborn 1893). Lemström carried out many systematic experiments and got the positive effects. In his report, the harvestings of barley, potato and radish increased 35, 76 and 60\%, respectively, at the cultivation under several tens highvoltage lines (Lemström 1904). Shibusawa also carried out various experiments on influence of electric field on plant growth using dc and ac high voltage for nine years from 1921 (Shibusawa and Shibata 1927). In the experiments, a copper mesh was used as electrode applied high voltage by power supply. The high voltage mesh electrode was located $15-30 \mathrm{~cm}$ upper from the plants, as shown in Fig. 2. They used various plants: corn, buckwheat, pea, wheat, burdock, soybean, green onion, Japanese radish and tabaco in the experiment. The got positive effect i.e., growth enhancement for buckwheat and tabaco. They also reported that germination of the winter bud of many kinds plant such as Cornus controversa, Prunus persica and Chaenomeles speciosa was promoted, as shown in Fig. 3 (Shibusawa and Shibata 1927).

The influence of electric field on plant growth changes by the electromagnetic energy feeding to the plant and the cultivation field. Murr reported that the response in plant growth against external electric field appeared over the criterial amplitude of the applied electric field (Murr 1965). The plant growth was enhanced around the optimum condition as shown in Fig. 4. However, the plant growth was suppressed by applying electric field which amplitudes was larger than $60 \mathrm{kV} / \mathrm{m}$. Similar results were reported by some researchers (Barman and Bhattacharya 2016). The criterial condition for suppression of the plant growth was determined by current flowing

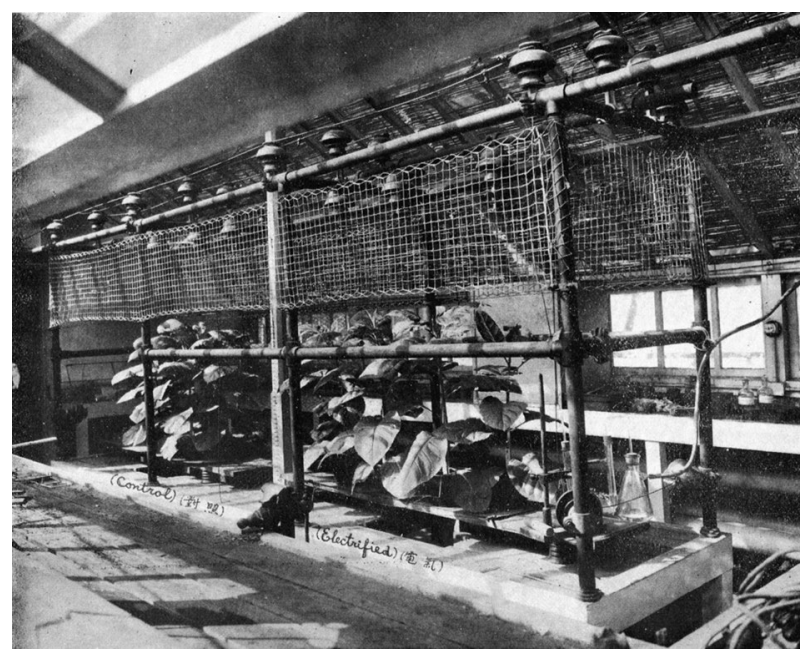

Fig. 2 Experimental setup for plant growth under applying electric field condition (Shibusawa and Shibata 1927) 
Fig. 3 Germination promotion of winter bud by apply
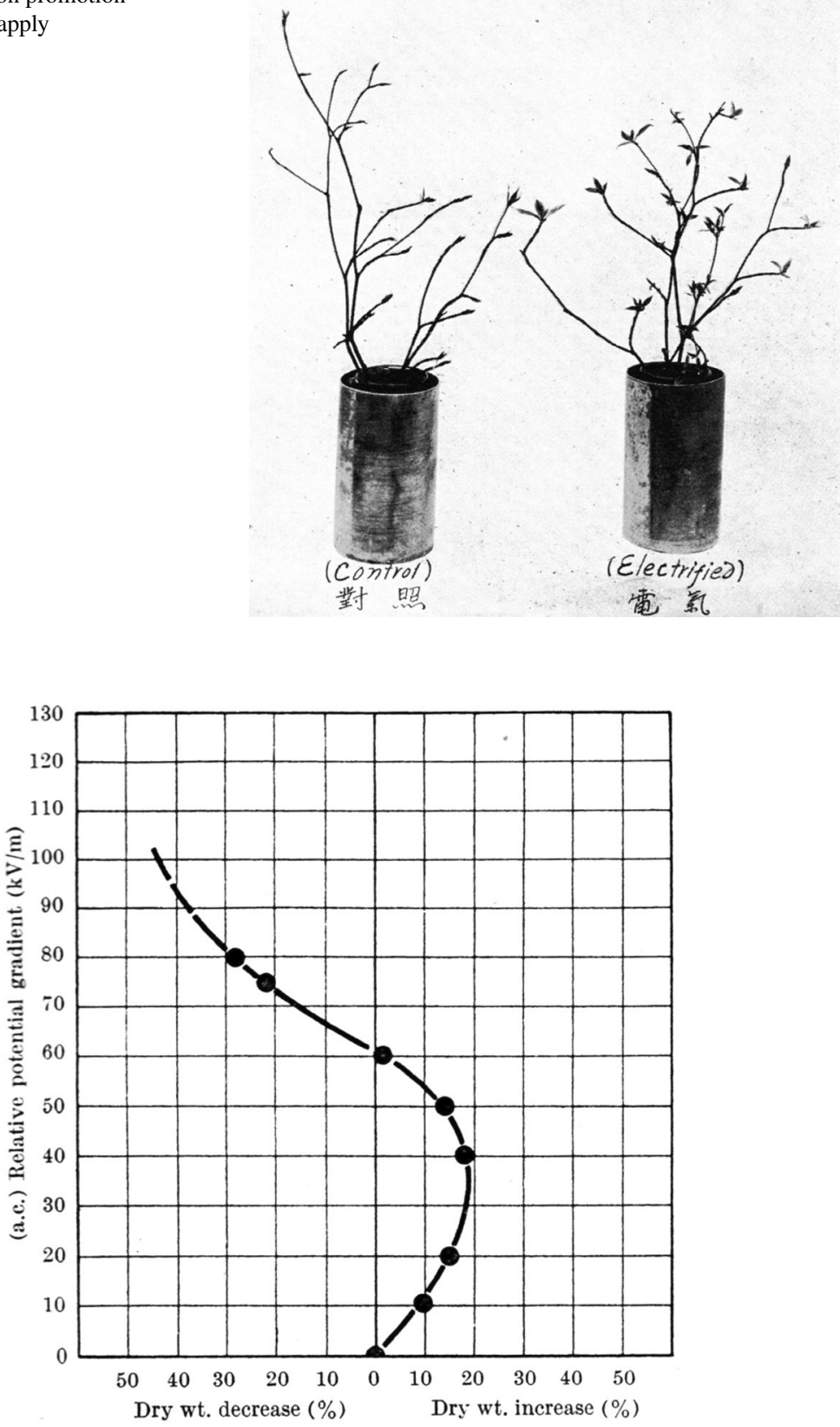

Fig. 4 Yellow bush bee leaf response based on an average of two dry weight comparisons of activated leaves at each electric field and control leaves (Murr 1965) 
into the plant body. Murr reported that the plant leaves were changed morphologically i.e., destroyed at more than $10^{-5} \mathrm{~A}$ of current. In range between $10^{-6}$ and $10^{-8}$ A, the electric field works as negative effect for the plant growth. In range between $10^{-9}$ and $10^{-15} \mathrm{~A}$, the electric field works as positive effect, i.e., enhancing the plant growth and improving the harvesting yield. The electric field did not affect the plant growth (non-response) when the current was lower than $10^{-16}$ A (Murr 1966). Especially, the corona discharge appeared when the applied electric field was higher than several tens $\mathrm{kV} / \mathrm{m}$ as shown in Fig. 5 (Shigemitsu 1999). This corona discharge damaged the plant leaves and other parts of body. The high voltage had applied to the plants and cultivation bed soil with various strength and conditions for more than 250 years by many researchers as reported by Barman and Bhattacharya, shown in Table 1 (Barman and Bhattacharya 2016).

\section{Basis of high voltage generation}

The high-voltage power supply including pulsed power generator is used to produce the intense electric field, accelerate electron and discharge plasma, which includes chemically active species; ROS and RNS (Weltmann et al. 2010; Graves 2012; Braithwaite 2000). Here, the generation of direct (DC) voltages, alternating (AC) voltages and transient voltage (pulsed power) is outlined as basis of high voltage generation.

\subsection{Direct voltages}

DC high voltages have a long history in developing the system and applications in science and industry (Kuffel et al. 2000). In the applied physics society, DC high voltage has been utilized in accelerators, electron microscopy, etc. DC high voltage have been used in electromedical equipment such as X-ray sources, also in industrial applications such as electro precipitator, electrostatic painting and powder coating.

In principle, the $\mathrm{DC}$ voltages are produced by rectifying $\mathrm{AC}$ voltage, as shown in Fig. 6. The AC low voltage is amplified by transformer TR as dotted line in Fig. 6b. If the impedance of diode $\mathrm{D}$ and leakage reactance of transformer TR

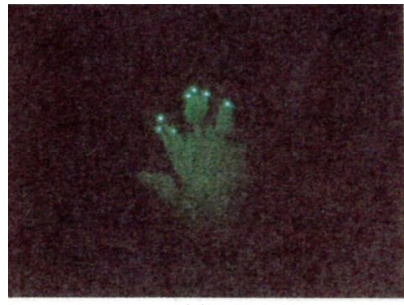

(a)

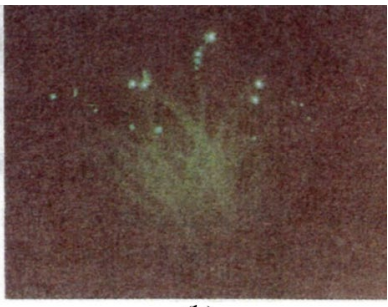

(b)

Fig. 5 Light emission of corona discharge on the leaves tip a of tulip (50 kV/m) and $\mathbf{b}$ of aloe $(40 \mathrm{kV} / \mathrm{m})$ (Shigemitsu 1999) 


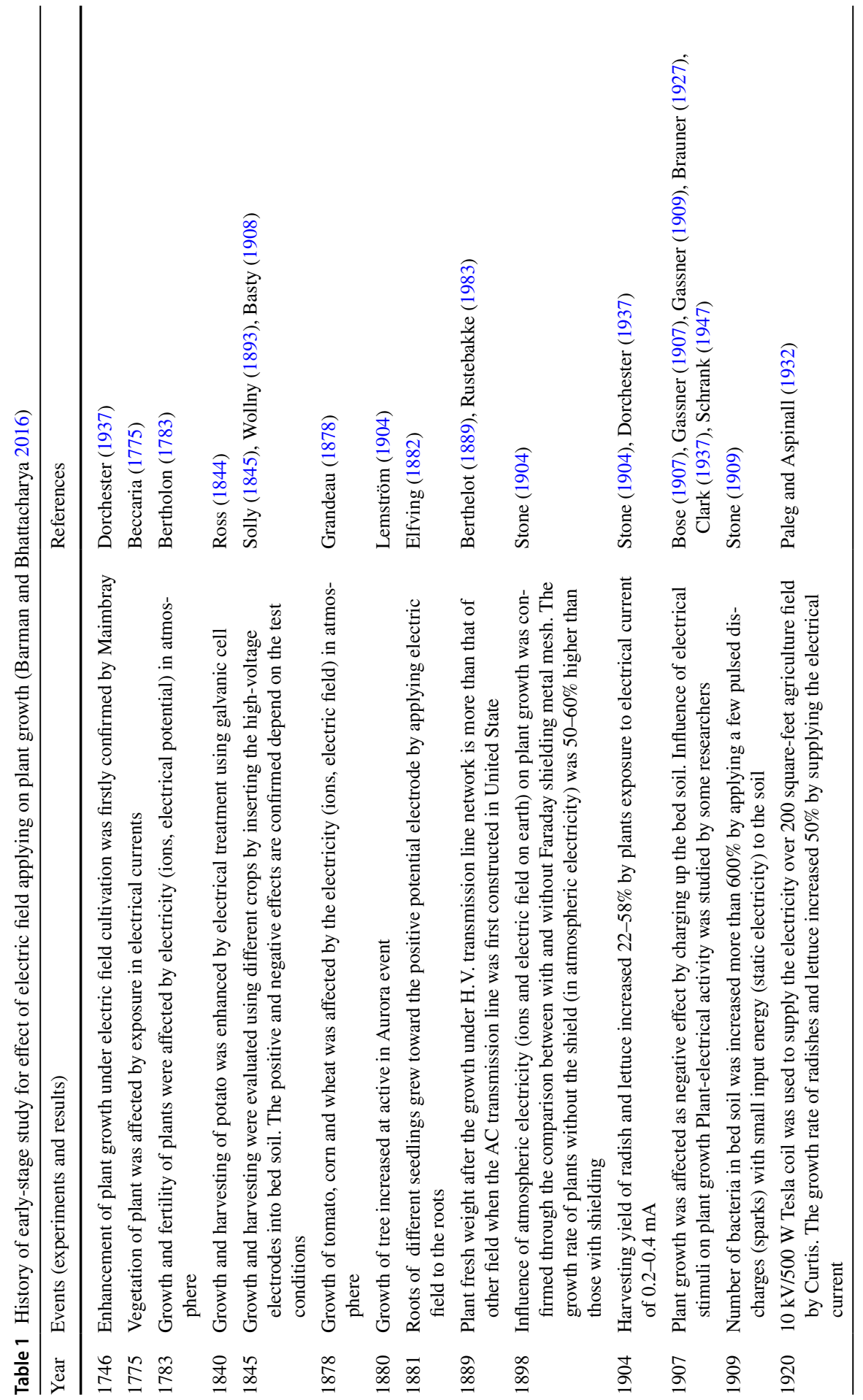




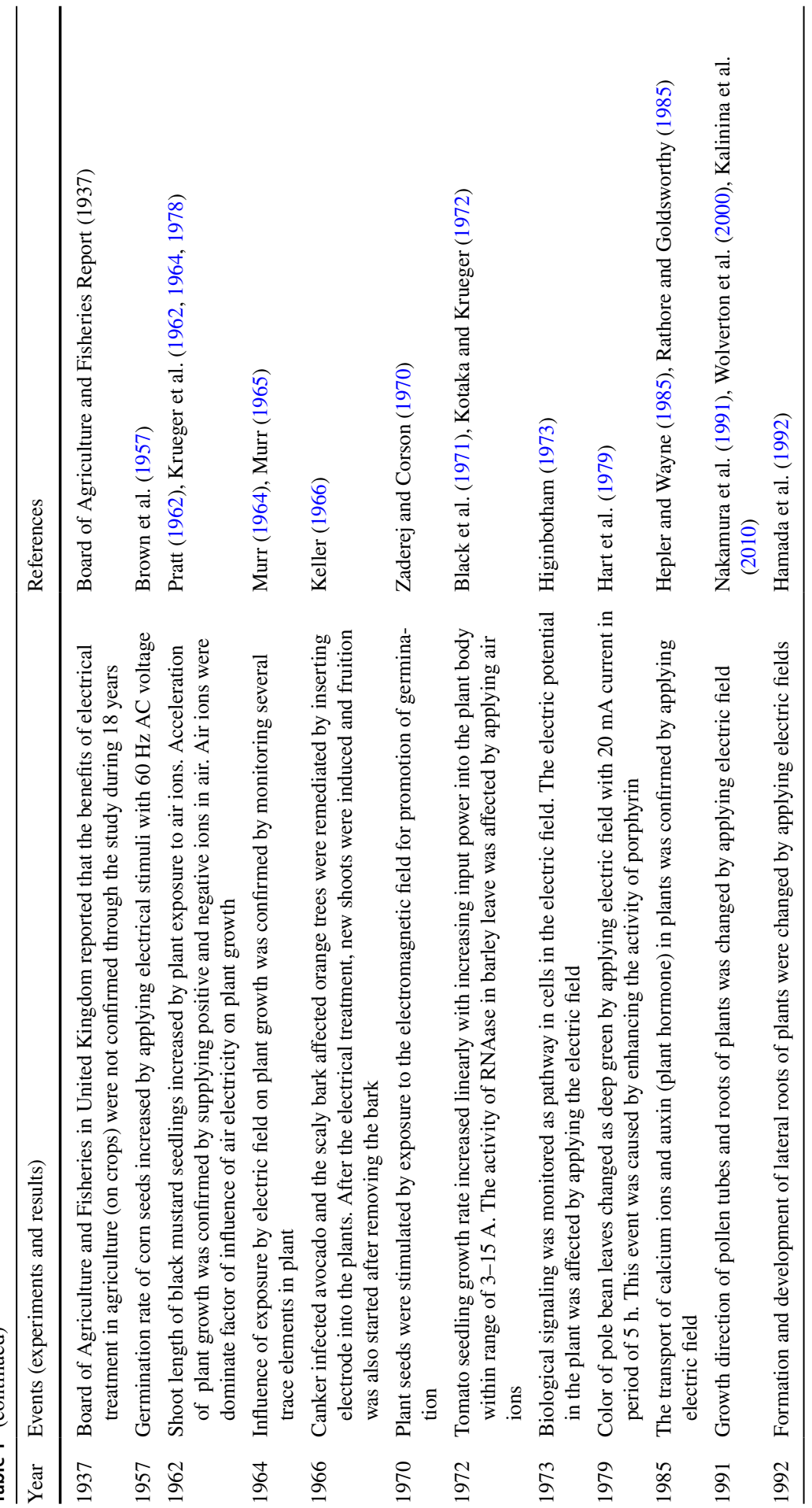




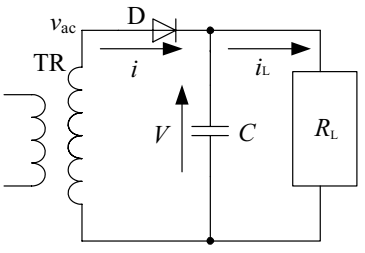

(a)

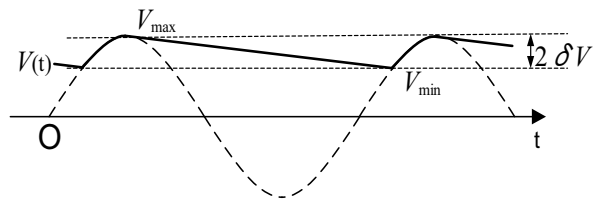

(b)

Fig. 6 AC/DC converter circuit consists of single-phase half-wave rectifier and smoothing capacitor; a circuit, b output voltages with connection of load $R_{\mathrm{L}}$

are negligible during conduction phase of the diode $D$, the capacitor $C$ is charged up to the peak (maximum) voltage $V_{\max }$ of the amplified AC voltage $V(t)$. If the load is open $\left(R_{\mathrm{L}}=\infty\right)$, the load current is zero $\left(i_{\mathrm{L}}=0\right)$. In this case, the voltage of capacitor $C$ keeps constant with value of $V_{\max }$ in spite of AC voltage $v_{\text {ac }}$ oscillates between $\pm V_{\max }$ as shown in Fig. 6b. When the resistor $R_{\mathrm{L}}$ is connected as a load, the output voltage does not remain as constant value $V_{\max }$. During one period $(T=1 / f ; f$ frequency of AC voltage), a charge stored in the capacitor is discharged to the load $R_{\mathrm{L}}$. As the result, the output voltage includes ripple, as shown in Fig. 6 b.

For the demands of very high DC voltages in some applications, the rectifier and capacitor units are sometimes used with cascade called as "Cockcroft-Walton type" circuit (Kuffel et al. 2000). The cascade connection high-voltage circuit was published by Greinacher in 1920, and after that the circuit was improved in 1932 by Cockcroft and Walton to produce high-energy positive ion. A six-stage cascade circuit of the "Cockcroft-Walton type" is shown in Fig. 7. The capacitor $C_{1}$ is charged up to $V_{\max }$ through a half-wave rectifier circuit, when the potential at node A reaches to the lowest potential of $-V_{\max }$. In the next half cycle, a halfwave rectifier of $D_{2}$ conducts. As the result, the potential of node $\mathrm{B}^{\prime}$ increases and reaches to $2 V_{\max }$ after one period of $\mathrm{AC}$ voltage cycle $(T=1 / f ; f$ is frequency).

Fig. 7 Cascade circuit according to Cockcroft-Walton

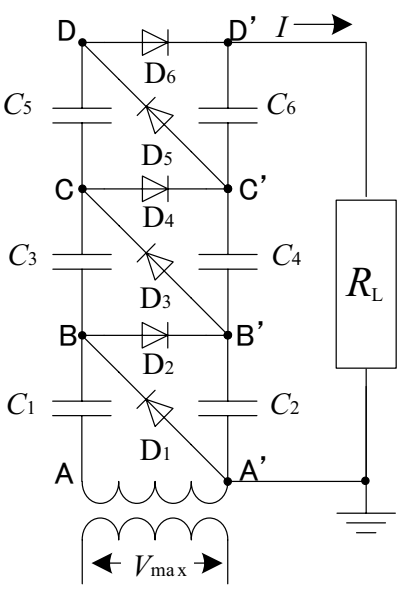


Therefore, all capacitors are charged up to $V_{\max }$ in the steady state and the potential of point $\mathrm{D}^{\prime}$ reaches $6 V_{\max }$. Extremely high voltage can be easily generated using several stages arranged in same rectifier and capacitor circuits.

Figure 8 shows schematics and photograph of high-voltage power supply based on Cockcroft-Walton circuit (Green techno, Kanagawa, Japan; GM100) for agricultural applications (Takahashi et al. 2018). The circuit consists of an AC/DC converter, a DC/AC converter, 12 stages of rectifier with capacitors. The output voltage is applied to the charging $130-\mathrm{pF}$ capacitor through a $100-\mathrm{M} \Omega$ resistor. The charge accumulated in the $130-\mathrm{pF}$ capacitor is transferred to the load through a spark gap switch. The DC/AC converter consists of a high-voltage transformer driven by a resonance circuit. The output voltage of the DC/AC converter is $6.2 \mathrm{kV}\left(8.7 \mathrm{kV}\right.$ in $\left.V_{\max }\right)$ with $25 \mathrm{kHz}$ in frequency. This output voltage is amplified by 12 -stage $\mathrm{C}-\mathrm{W}$ circuit to almost $100 \mathrm{kV}$ as voltage of the charging capacitor. The AC/DC and DC/AC converters, $\mathrm{C}-\mathrm{W}$ circuit, the charging capacitor and the charging resistor are inside of the box, as shown in Fig. 8b. The circuit is molded by a resin for insulation in the box. Typical example of output voltage of the $\mathrm{C}-\mathrm{W}$ circuit is shown in Fig. 9. The potential of charging capacitor recovers almost $230 \mathrm{~ms}$ after a discharge of all charge in the capacitor by turning the spark

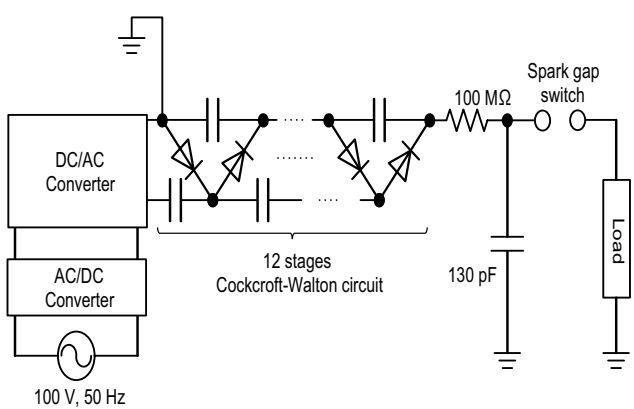

(a)

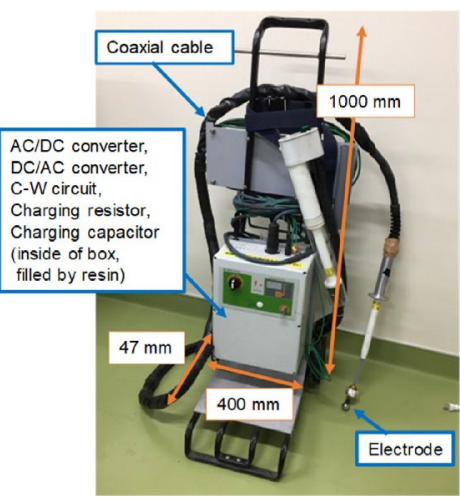

(b)

Fig. 8 Cockcroft-Walton (C-W) circuit (a) and photograph (b) of C-W power source for agricultural applications

Fig. 9 Waveforms of output voltage of $\mathrm{C}-\mathrm{W}$ circuit with repetitive spark as load

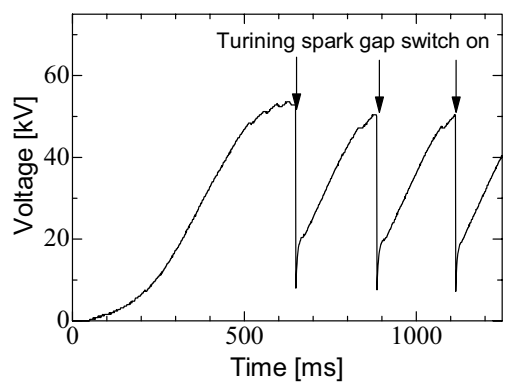

Springer 
gap switch on. The recovery time is limited by the impedance of converters and capacitance of the $\mathrm{C}-\mathrm{W}$ circuit.

\subsection{Alternating voltages}

$\mathrm{AC}$ high voltage has also long history because the AC high voltage was chosen as electric power transmission in the transmission and distribution systems. Then, the most studies and tests in electrical insulation systems have been carried out using $\mathrm{AC}$ high voltages at the nominal frequency of the test objects. AC high voltage is commonly generated using single-phase transformer optimized to the suitable working frequency. Figure 10 shows illustration and diagram of single-phase transformer. The transformer consists of primary winding $n_{1}$, secondary winding $n_{2}$ and core, which works as a magnetic flux circuit. The primary winding $n_{1}$ is usually rated for low voltages of $V_{1}<1 \mathrm{kV}$, however, often be split up into two or more windings which can be connected in series of parallel to increase the capabilities. The core is made of iron for low frequency or ferrite for high frequency. The core is grounded and one terminal of each of the two windings is also grounded to fix the ground potential. The secondary winding $n_{2}$ is usually rated for high voltages $V_{2}$. The secondary output voltage and current are simply expressed as:

$$
V_{2}=\frac{n_{2}}{n_{1}} V_{1}, \quad I_{2}=\frac{n_{1}}{n_{2}} I_{1} .
$$

\subsection{Impulse voltages}

Disturbances of electric power transition and distribution systems are frequently caused by two kinds of transient voltages which have amplitudes much higher than the peak values of the normal AC operation voltage. The first kind is lightning overvoltage, which is caused by lightning strokes hitting the phase wires of overhead lines. The second kind is switching overvoltage which is caused by switching phenomena in electrical power distribution system. The actual shape of both kinds overvoltage varies by each situation. However, it becomes necessary to simulate these transient voltages by relatively simple shapes for evaluation the power components in testing. For most applications of lightning impulse voltages, the front time $T_{1}$ of $1.2 \mathrm{~ms}$ and the time to half-value $T_{2}$ of $50 \mathrm{~ms}(1.2 / 50$ impulse) are commonly

Fig. 10 Single-phase transformer, a schematics and $\mathbf{b}$ diagram

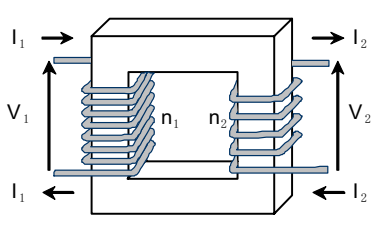

(a)

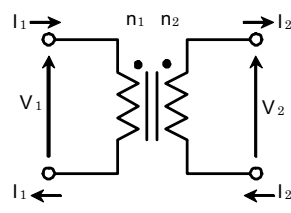

(b) 
employed as standard lightning impulse regulation. For switching impulse voltages, the time to peak $T_{\mathrm{P}}$ of $250 \mathrm{~ms}$ and the time to half-value $T_{2}$ of $2500 \mathrm{~ms}(250 / 2500$ impulse) are employed.

In general, extremely high voltage such as $1 \mathrm{MV}$ is required for the purpose of testing the power transfer and distribution elements. However, there are many difficulties (e.g., spark gaps for switching of very high voltages, the increase of the physical size of the circuit elements, high DC voltages to charge capacitor, suppressing corona discharges during the charging period) for making the extremely high voltage with one-stage. An idea to overcome the above-mentioned difficulties was suggested as new arrangement in which a number of capacitors were charged in parallel and then changing the connection in series through the spark gap switches by Marx in 1923. Figure 11 shows schematic of three-stage Marx generator. The three capacitors $\mathrm{C}$ are connected in parallel and are charged up to $V_{\text {in }}$ through charging resistors $R$. After the charging the capacitors up, the lowest spark gap GS1 is fired by trigger ignitor which is followed by an almost simultaneous breakdown of all remaining spark gap switches GS2 and GS3. As the result, the connection of the capacitors changes from parallel to series, then voltage of $-3 V_{\text {in }}$ is supplied with a polarity opposite to that of the charging voltage.

Figure 12a shows basic circuit for single-stage impulse generators. The capacitor $\mathrm{C}$ is charged up with a $\mathrm{DC}$ power supply through a charging resistor $R_{\mathrm{C}}$. After the charging the capacitor up, the spark gap switch is turned on by firing the gap switch with ignitor. An ignition time of the spark gap switch is much shorter than the front time $\left(T_{1}\right)$. After the gap switch is closing, the output voltage between the resistance $R$ can be roughly expressed as shown in Fig. $12 \mathrm{~b}$ at $\left(\frac{R}{L}\right)^{2}-\frac{4}{L C} \gg 0$. The time constants for rise and fall of output voltage are roughly estimated as $L / R$ and

Fig. 11 Schematics of threestage Marx circuit

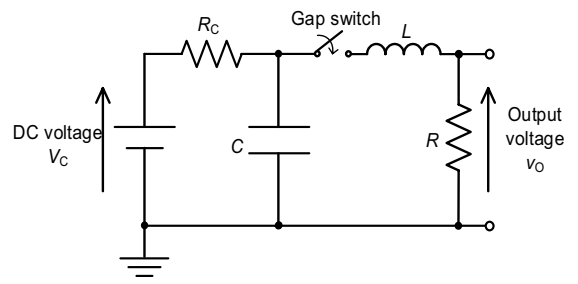

(a)
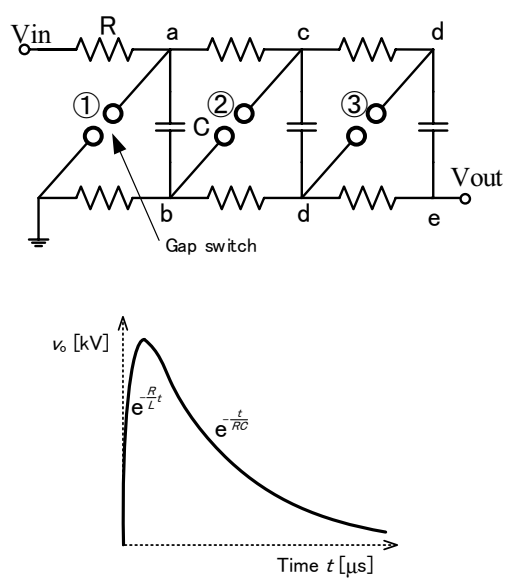

(b)

Fig. 12 Single-stage impulse generator; a circuit, $\mathbf{b}$ waveform of output voltage at $\left(\frac{R}{L}\right)^{2}-\frac{4}{L C} \gg 0$ 


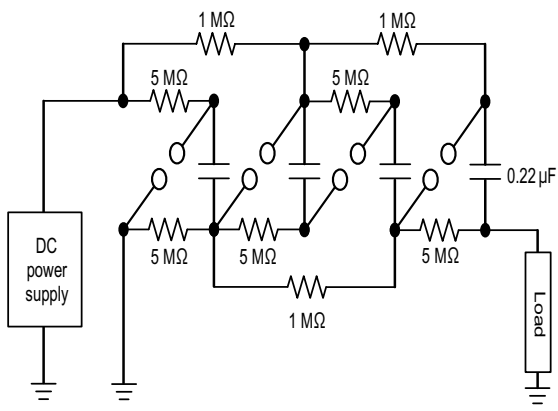

(a)

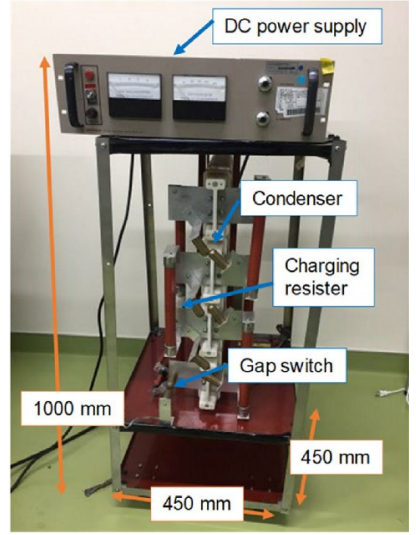

(b)

Fig. 13 Schematic (a) and photograph (b) of four-stage Marx circuit for agricultural applications

$R C$, respectively, under the condition of $\left(\frac{R}{L}\right)^{2}-\frac{4}{L C} \gg 0$. Therefore, we can control the waveform with choosing values of resistance $R$, capacitance $C$ and inductance $L$.

Figure 13 shows the schematic and photograph of Marx circuit for agricultural applications (Takaki et al. 2009; Takahashi et al. 2018). The Marx generator consists of four $0.22 \mu \mathrm{F}$ capacitors (Maxwell, 31,160), charging resistors (1 and $5 \mathrm{M} \Omega$ ) and the spark gap switches. The capacitors are charged up using a DC high-voltage power supply up to $12.5 \mathrm{kV}$. The charging time is approximately $10 \mathrm{~s}$ because of the output current limitation of the DC charging unit (power supply). When a spark gap switch is closed, the remaining gas switches are sequentially closed automatically, and the connection of capacitors is changed from parallel to series. The size and weight of the Marx generator are $1.0 \mathrm{~m} \times 0.45 \mathrm{~m} \times 0.45 \mathrm{~m}$ and $39.4 \mathrm{~kg}$, respectively. It can be used in agricultural field by carrying the generator using van-type car.

\subsection{Generation of pulsed power}

Pulsed power is technology of accumulating energy during relative long period of time and of releasing the accumulated energy in extremely short period as highpower pulse composed of high voltage and large current, however, moderate low energy, i.e., low time-averaged power (Lehr and Rpn 2017). Pulsed power is generated by transferring an energy stored in capacitors or inductors to a load in extremely short time using switching devices (Bluhm 2006). The pulsed power technologies have been used for internal fusion, high-power microwaves and lasers, electrothermal launcher, particle accelerators, etc. The applications of pulsed power technologies are still expanded in fields including environment, material processing, medical treatment, food processing and agriculture (Bluhm 2006). There are some books and review papers about generation of pulsed power including nanosecond pulses (Martin 1992; Mankowski and Kristiansen 2000; Smith 2002; Mesyats 2007). Recently 
Akiyama (Akiyama and Heller 2017) in chapter 2, Huiskamp (Huiskamp 2020) for a detailed overview.

Pulsed power applications for biology have been newly developed, in which both high repetition and performance are required by researchers and scientists because of optimizing to the load of living organisms (Akiyama and Heller 2017). This section summarizes the most common methods of generating microsecond and nanosecond pulses with a focus on this new application field. The aim of this section is to show the typical pulsed power sources as basis of choosing optimum power supply for each application in agriculture and food processing.

\subsubsection{Basic circuit for pulsed power}

Figure 14 shows familiar circuits combining a capacitor of an inductor and switches. Capacitors and inductors (reactive elements) are used as primary energy storage elements in the form of electric field $\left(0.5 e E^{2}\left[\mathrm{~J} / \mathrm{m}^{3}\right]\right.$, where $e$ dielectric constant, $E$ electric field strength) and magnetic fields $\left(0.5 m H^{2}\left[\mathrm{~J} / \mathrm{m}^{3}\right]\right.$, where $m$ magnetic permeability, $H$ magnetic field strength), respectively.

In the capacitor-resistor circuit (capacitive energy storage system) shown as Fig. 14a, the electrical energy $0.5 C V_{0}^{2}$ ( $V_{0}$ initial charging voltage) is stored in a capacitor and then transferred to a load resistor $R_{\mathrm{L}}$ through a closing switch $\mathrm{S}$. The load voltage and current after closing the switch $\mathrm{S}$ are obtained as follows using continuity of current in the circuit.

$$
\begin{aligned}
& v(t)=V_{0} \exp \left(-t / R_{L} C\right), \\
& i(t)=\frac{V_{0}}{R_{L}} \exp \left(-t / R_{L} C\right),
\end{aligned}
$$

where $t$ time after closing switch $S$.

In the inductor-resistor circuit (inductive energy storage system) shown as Fig. 14b, the magnetic energy $0.5 \mathrm{LI}_{0}{ }^{2}$ ( $I_{0}$ initial current in the inductor) is stored in an inductor and then transferred into a load resistor $R_{\mathrm{L}}$ by opening switch $\mathrm{S}_{1}$ and closing switch

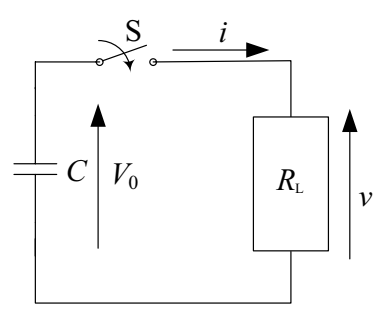

(a)

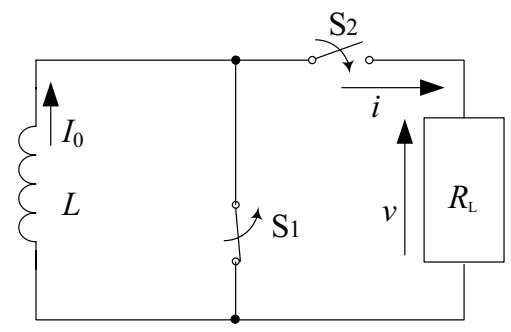

(b)

Fig. 14 Basic circuits for pulsed power; $\mathbf{a}$ capacitive and $\mathbf{b}$ inductive energy storage system 
$\mathrm{S}_{2}$. The load voltage and current after closing switch $\mathrm{S}_{2}$ are obtained as follows using Kirchhoff's voltage law:

$$
\begin{gathered}
v(t)=R_{\mathrm{L}} I_{0} \exp \left(-\frac{t}{L / R_{\mathrm{L}}}\right), \\
i(t)=I_{0} \exp \left(-\frac{t}{L / R_{\mathrm{L}}}\right) .
\end{gathered}
$$

Figure 15 shows (a) the circuit diagram, and (b) typical output voltage of the inductive energy storage system pulsed power generator for driving non-thermal plasma reactors (Takaki et al. 2007, 2011; Takahashi et al. 2011). The electrical charges stored in the capacitor $\mathrm{C}_{1}$ are transferred to the pulse transformer by sparking the gap switch. The primary voltage $V_{1}$ of the pulse transformer is amplified theoretically five times owing to the ratio of primary and secondary windings of the pulsed transformer. The amplified voltage is outputted as secondary voltage of the pulse transformer, and then applied to the inductor $L(2.5 \mathrm{mH})$ and capacitor $\mathrm{C}_{2}(1 \mathrm{nF})$. As the result, the current flows through the diode with LC oscillation as shown in Fig. 15b. In this circuit, fast recovery diodes (Voltage multiplier, K100UF) are employed as a semiconductor opening switch (SOS). After the current direction reverses with LC resonance, the electrical charges are injected into the SOS during $100 \mathrm{~ns}$ reverse time. The SOS diode recovers after the charge injection phase, and then the current is interrupted in very short time. As the result, a high-voltage pulse is generated like surge voltage (inductive voltage) by the short time circuit current interruption as follows:

$$
v_{\mathrm{o}}(t)=-v_{2}(t)+\frac{1}{C_{2}} \int i_{\text {sos }} \mathrm{d} t+L \frac{\mathrm{d} i_{\text {sos }}}{\mathrm{d} t} \cong L \frac{\mathrm{d} i_{\text {sos }}}{\mathrm{d} t} .
$$

The typical waveforms of the circuit current, capacitor voltage and output voltage are shown in Fig. 15b. The pulse voltage with $27 \mathrm{kV}$ peak amplitude and $60 \mathrm{~ns}$ width (FWHM) is obtained using the IES circuit. An IES circuit is classified as a type of voltage amplifier caused by a short time interruption of a circuit

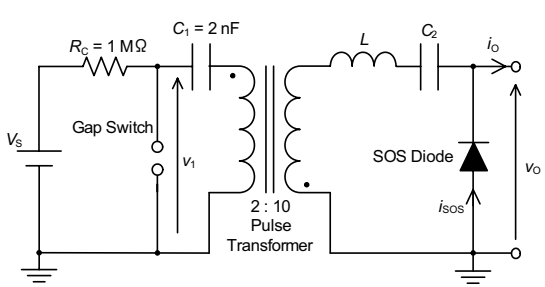

(a)

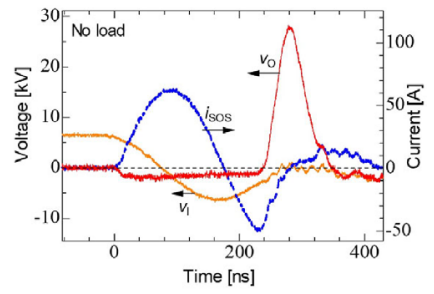

(b)

Fig. 15 Basic circuits for pulsed power; $\mathbf{a}$ capacitive and $\mathbf{b}$ inductive energy storage system 
current using an opening switch. In IES, the timing of opening the switch to interrupt the current is important because an output voltage strongly depends on the amplitude of the current just prior to the interruption. In general, the opening switch is designed to operate at just prior to peak of the circuit current (Rukin 1999; Teramoto et al. 2001).

\subsubsection{Single pulse-forming line pulse source}

Most applications for pulsed power require a constant voltage during period of pulse width. However, the output voltage is critically damped using the pulsed power generator with a single capacitor and inductor. The output voltage waveforms can be changed from critically dumping (double-exponentials shape) to square (constant in arbitrary period) by pulsed power sources with multiple elements; such circuits are called pulse-forming networks (PFNs), whose transmission line (distributed constant circuit) is also used as pulse-forming lines (PFLs). In this section, pulse transmission lines by a lumped element description are analyzed using the Maxwell equations for understanding basic behavior of single pulse-forming line pulse source. Discrete elements PFNs are treated in Sect. 3.4.4.

Figure 16a shows the diagram of a transmission line as distribution circuit with differential elements of length $\mathrm{dz}$. Figure $16 \mathrm{~b}$ shows its model using lamped circuit elements $L \mathrm{~d} z$ and $C \mathrm{~d} z$, where $L[\mathrm{H} / \mathrm{m}]$ and $C[\mathrm{~F} / \mathrm{m}]$ are inductance and capacitance per unit length, respectively. Figure 17 shows typical transmission lines. Based on Kirchhoff's voltage and current laws, the voltage and current

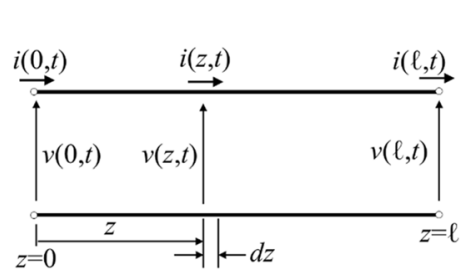

(a)

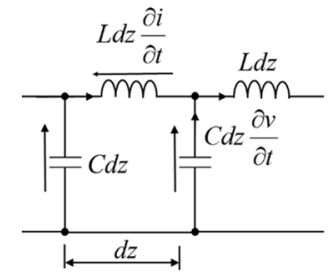

(b)

Fig. 16 Transmission line; $\mathbf{a}$ distributed circuit and $\mathbf{b}$ its lumped circuit element analogue

Fig. 17 Typical transmissions lines

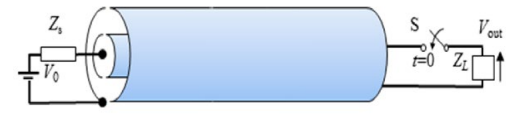

(a) coaxial transmission line

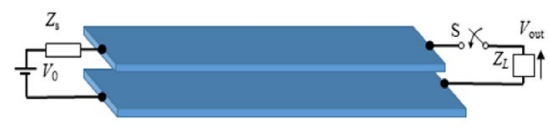

(b) strip line 
differences between two points can be expressed using contentious partial differential equations when $\mathrm{d} z$ is small,

$$
\frac{\partial v}{\partial z}=-L \frac{\partial i}{\partial t}, \quad \frac{\partial i}{\partial z}=-C \frac{\partial v}{\partial t} .
$$

These telegraphist's equations can be combined to give wave equations for voltage and current as follows,

$$
\frac{\partial^{2} v}{\partial t^{2}}=\left(\frac{1}{L C}\right) \frac{\partial^{2} v}{\partial z^{2}}, \quad \frac{\partial^{2} i}{\partial t^{2}}=\left(\frac{1}{L C}\right) \frac{\partial^{2} i}{\partial z^{2}} .
$$

These equations are mathematical expressions of the properties of a transmission line. Therefore, the voltage and current in the transmission line can be easily obtained as follows,

$$
\begin{gathered}
v(z, t)=v_{\mathrm{f}}(z-v t)+v_{\mathrm{b}}(z+v t), \\
i(z, t)=\frac{1}{Z_{0}}\left\{v_{\mathrm{f}}(z-v t)-v_{\mathrm{b}}(z+v t)\right\},
\end{gathered}
$$

where $v_{\mathrm{f}}$ and $v_{\mathrm{b}}$ are forward andackward voltage waves, respectively, $Z_{0}$ characteristic impedance of line $\left(Z_{0}=\sqrt{L / C}\right)$ and $v$ phase velocity $(v=1 / \sqrt{L C})$. The capacitance $C$ and inductance $L$ can be calculated geometry of the transmission lines. The phase velocity is expressed as using geometrically obtained $L$ and $C$ values as follows,

$$
v=1 / \sqrt{\epsilon \mu} .
$$

Figure 18 shows snapshots of the voltage distribution in the transmission line and the output voltage when the end of the line is connected to a load of an impedance $Z_{\mathrm{L}}$. The reflection coefficient $k$ at transmission line end is

$$
k=\frac{Z_{\mathrm{L}}-Z_{0}}{Z_{\mathrm{L}}+Z_{0}} .
$$

The above equation indicates that the condition of $Z_{\mathrm{L}}=Z_{0}$ results in $k=0$, i.e., no reflection and all electromagnetic energy is transferred in the load. This situation (no reflection) is called a matching between load and transmission line. In the matching load case, the voltage of $V_{0} / 2$ ( $V_{0}$ is the charging voltage to pulse-forming line) is applied to the load with pulse width of $2 l / v, l$ is the length of pulse-forming line and $v$ phase velocity. For commercially available coaxial cables, $Z_{0}$ is almost always 50 $\Omega$ and the phase speed $v$ of waves is typically $5 \mathrm{~ns} / \mathrm{m}$. 


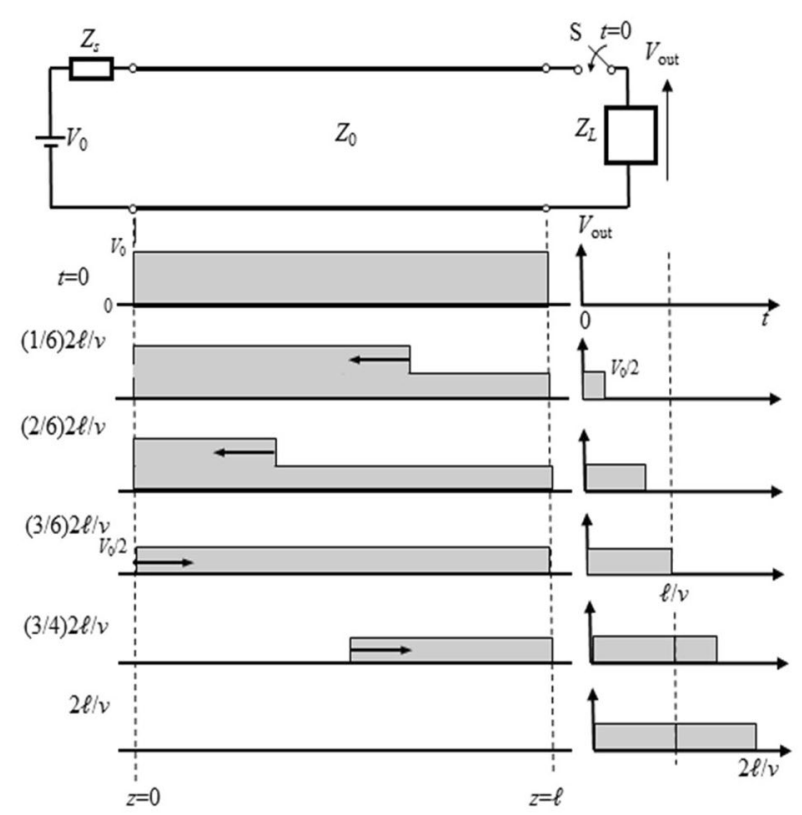

Fig. 18 Snapshots of the voltage distribution in the line and the output voltage at $Z_{0}=Z_{\mathrm{L}}$

\subsubsection{Blumlein-line pulse source}

A disadvantage of the single-line pulse transformer is that the output voltage is only half of the charging voltage. The Blumlein-line pulse source consists of two single forming lines slightly complicated connection and can solve a problem of the disadvantage. Figure 19 shows schematic of the Blumlein-line pulse source. Two pulse-forming lines both are connected to the load and other side is floating between the lines. The equivalent impedance from the load is obtained as $2 Z_{0}$. Then, the load impedance has to be chosen as $Z_{\mathrm{L}}=2 Z_{0}$ for matching condition, i.e., without reflection of the voltage wave. Both lines are charged through a high impedance $Z_{\mathrm{s}}$ and inductor $L_{\mathrm{C}}$ to a voltage of $V_{0}$. At $t=0$ switch $\mathrm{S}$ closes, then reflection coefficient of the PFL-1 end changes from 1 to -1 . Therefore, the polarity of the voltage $v_{1}$ changes from positive to negative and propagates towards the load. When the voltage wave propagates through a transmission line with impedance $Z_{0}$ and it reaches to a load with impedance $Z_{\mathrm{L}}$, amplitude of $0.5 V_{0}$ is reflected as backward traveling wave because the reflection coefficient is obtained as 0.5 using Eq. (12) at $Z_{\mathrm{L}}=2 Z_{0}$. The whole events are graphically shown in Fig. 18. The voltage between the load can be obtained as differential of $v_{1}$ and $v_{2}$ at the load side end. The pulse voltage appears after a $l / v$ delay with duration of $2 \mathrm{l} / \mathrm{v}$ and an amplitude of $V_{0}$. Therefore, the Blumlein-line pulse source has the advantage that the output voltage is equal to the charging voltage (Huiskamp 2020). 


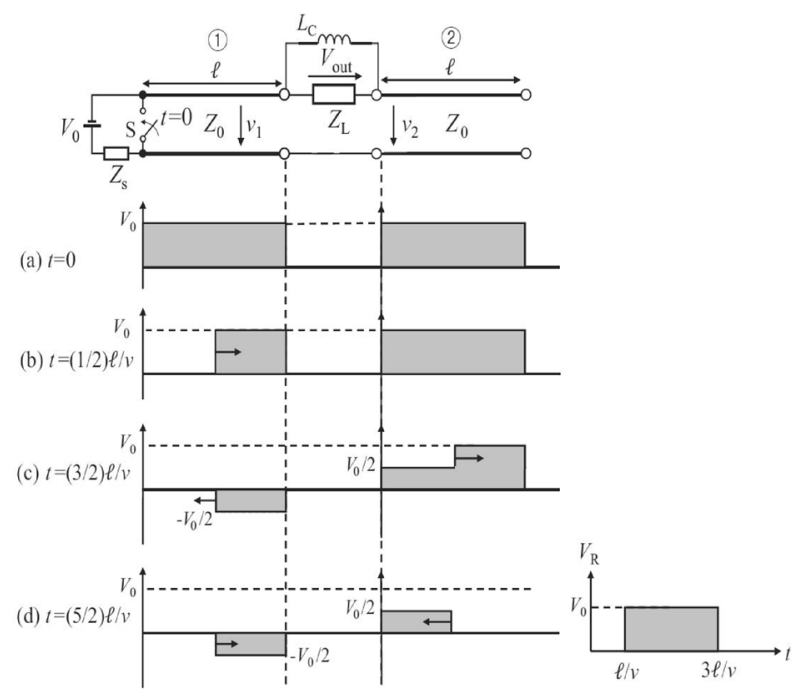

Fig. 19 Snapshots of the voltage distribution in Blumlein-line and the output voltage at $Z_{\mathrm{L}}=2 Z_{0}$

Figure 20 shows a three stacked Blumlein-line pulse generator using six coaxial cables lines (Namihira et al. 2000). On the primary side, these lines are connected in parallel, but on the load side, those lines are connected in series. This arrangement results in an output impedance of $300 \Omega$ at cable impedance of $50 \Omega$. The spark gap switch (SGS) is used as closing switch. The gap switch is controlled by triggering ignitor. Figure 21 shows the typical output voltage of the stacked Blumlein-line generator for different lengths of the coaxial cable (4, $6,8,10$ and $12 \mathrm{~m}$ ) with a corona discharge plasma reactor as a load. The pulse width of output voltage changes by changing the cable length.

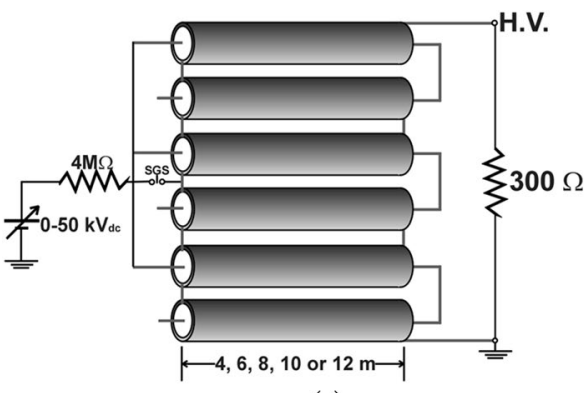

(a)

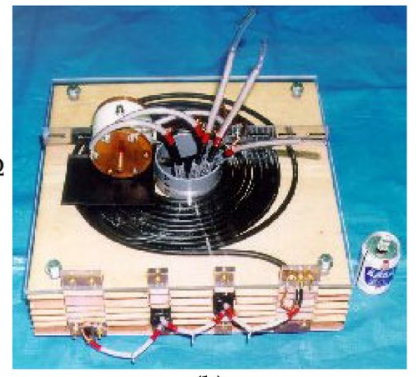

(b)

Fig. 20 Circuit diagram (a) and photograph (b) of three stacked Blumlein-line generator consisted of six coaxial lines (Namihira et al. 2000) 
Fig. 21 Output voltage of Blumlein-line for different lengths of cable with a plasma reactor as a load (Namihira et al. 2000)

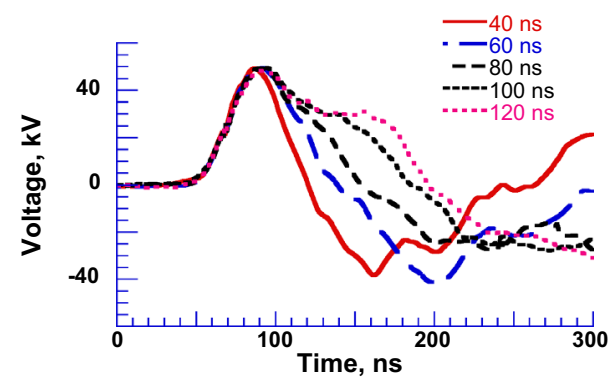

\subsubsection{Pulse-forming network pulse source}

Pulse-forming lines (PFLs) are generally used in many applications in time range from 5 to $200 \mathrm{~ns}\left(5 \mathrm{~ns}<\mathrm{D} t_{\mathrm{p}}<200\right)$. However, PFLs are impractical for pulse length longer than $1 \mathrm{~ms}$ because the cable length is longer than $1000 \mathrm{~m}$. For long pulse length more than $1 \mathrm{~ms}$, the discrete element circuits such as capacitors and inductors are commonly used to achieve better output waveforms. The discrete elements of capacitors and inductors have large capabilities (capacitance and inductance) compared with transmission line; therefore, they provide a shaped square waveform without critically damping. This pulse sources are called as pulse-forming networks (PFNs).

Figure 22 shows PFN circuit consisted of discrete elements of capacitors and inductors. The voltage and current traveling waves are express in same equations described in Sect. 3.4.2. PFN can be analyzed using a finite number $N$ of inductor-capacitor units instead of distributed LC components. As the result of analysis described in Sect. 3.4.2, the impedance of PFN is obtained as $Z_{0}=\sqrt{L / C}$, where the quantities $L$ and $C$ are the inductance and capacitance of discrete elements. The $Z_{0}$ is used to match with load, i.e., criterion of no-reflection at the load. The traveling time of voltage and current wave through the PFN is expressed as $N \sqrt{L C}$. Therefore, the output voltage pulse is obtained as amplitude of $V_{0} / 2$ and pulse duration of $\Delta t_{p}=2 N \sqrt{L C}$ at connecting resistor of $Z_{0}$ as a load.

Figure 23 shows the example of deigned PFN and its output voltage with connecting a load resistance of $Z_{0}=Z_{\mathrm{L}}$ (matching condition; no reflection at the end of PFN). The PFN consists of $2 \mathrm{nF}$ capacitor and $1.25 \mathrm{mH}$ with $N=10$. The charging voltage is $10 \mathrm{kV}$. The pulse length is calculated as

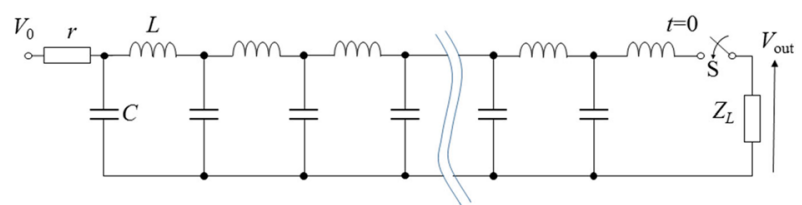

Fig. 22 Diagram of pulse-forming network 


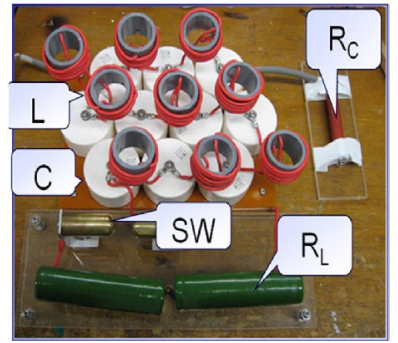

(a)

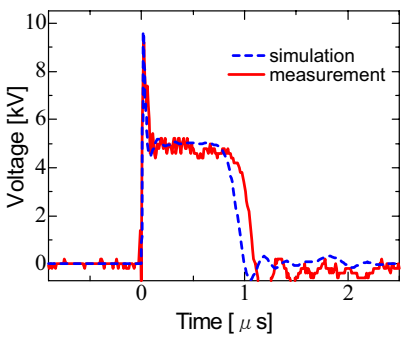

(b)

Fig. 23 Schematics of deigned PFN (a) and its output voltage (b) with connecting a load resistance of $Z_{0}=Z_{\mathrm{L}}$ at $C=2 \mathrm{nF}, L=1.25 \mu \mathrm{H}$ and $N=10$

$$
\Delta t_{\mathrm{p}}=2 \times 10 \sqrt{1.25 \times 10^{-6} \times 2 \times 10^{-9}}=1 \mu s .
$$

Figure 23 indicates that the pulse length and amplitude of output pulse are almost same values of calculating results of $1 \mathrm{~ms} 5 \mathrm{kV}\left(=V_{0} / 2\right)$.

\subsubsection{Generator using power semiconductor device}

For applications in the agriculture and food processing, compact and repetitive operation pulse sources are required. Semiconductor power switching devices such as insulated gate bipolar transistors (IGBTs), metal-oxide-semiconductor field-effect transistor (MOSFET) and semiconductor opening switch (SOS) are generally used to drive the pulse modulator with high repetition rate. The pulse voltage can be generated by direct switching of high-voltage DC between falling and rising phases. Figure 24a shows a schematic circuit which consists of AC/DC converter circuit, $\mathrm{H}$-bridge connected four IGBTs and pulse transformer which is used for amplifying voltage to $10 \mathrm{kV}$. The pulse width and the pulse repetition rate are controlled with timing of gate trigger of the semiconductor switching devices. Duty factor (ratio of

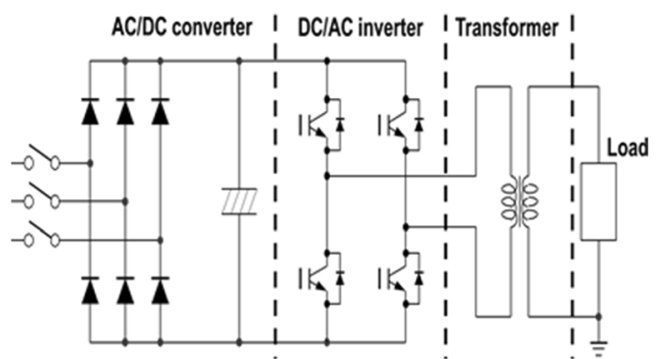

(a)

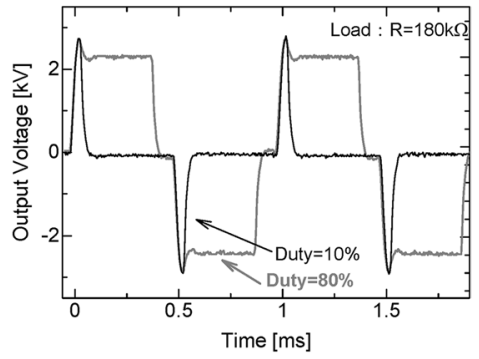

(b)

Fig. 24 Schematic of a circuit consisted of AC/DC converter, H-bridge with four IGBTs and pulse transformer and $\mathbf{b}$ its output voltage waveforms 
on time per pulse cycle) can be also controlled by the gate-trigger timing as shown in Fig. 24b (Miura et al. 2007).

Figure 25 shows $13 \mathrm{kV}$ silicon carbide ( $\mathrm{SiC}$ )-MOSFET-driven compact inductive energy storage (IES) pulsed-power generator (Takahashi et al. 2021). The rising and falling voltage rates of the SiC-MOSFET were 157 and $129 \mathrm{kV} / \mu \mathrm{s}$, respectively. The maximum current of the drain was $128 \mathrm{~A}$. The minimum on-resistance was obtained as $1.07 \Omega$. For an IES circuit, the output voltage and pulse width (FWHM) were obtained as $31.4 \mathrm{kV}$ and $55 \mathrm{~ns}$, respectively, at a charging voltage of $1100 \mathrm{~V}$, as shown in Fig. 26 (Takahashi et al. 2021). The maximum energy transfer efficiency was $50.2 \%$ at a load resistance of $2.5 \mathrm{k} \Omega$. The SiC-MOSFET-driven IES circuit has excellent performance as a compact pulsed power system in many industrial applications such as driving a corona plasma reactor for gas decomposition, water remediations, etc.

\subsubsection{Magnetic pulse compression}

Magnetic pulse compression (MPC) pulse sources had been developed to use in many applications such as driving high-power excimer lasers. In the MPC, the magnetic switch is key component to determine the pulse shape. A saturable inductor,

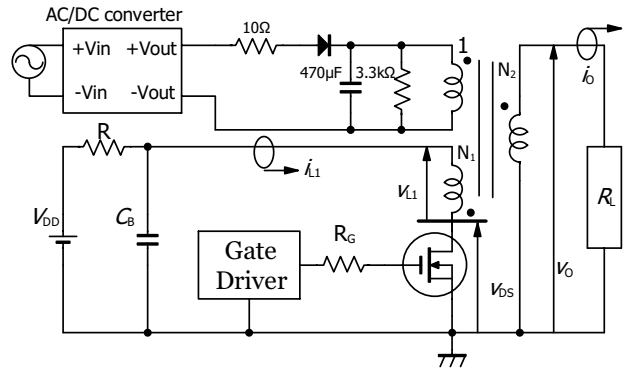

(a)

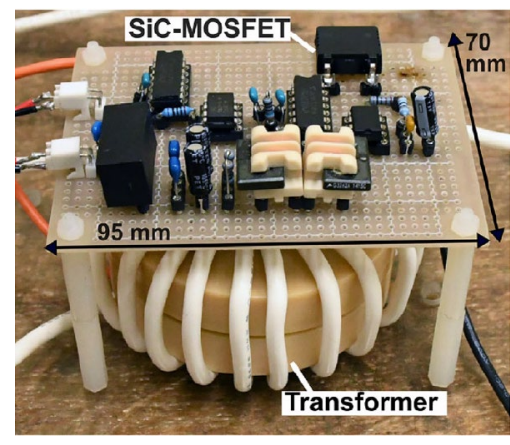

(b)

Fig. 25 Circuit diagram (a) and photograph (b) of compact inductive energy storage pulsed-power generator driven by a $13 \mathrm{kV}$ SiC-MOSFET

Fig. 26 Waveforms of the output voltage of secondary transformer for various input voltages

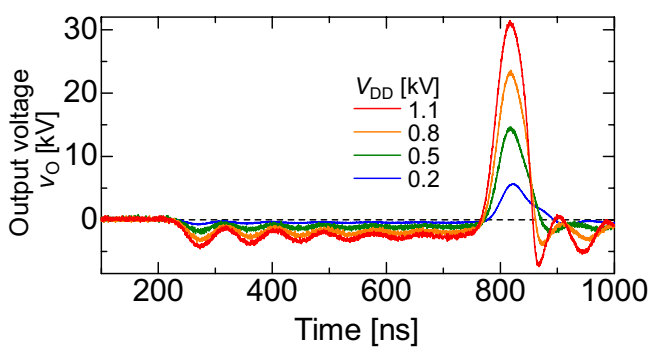


in which an inductor is wound on a magnetic core, is commonly used as magnetic switch in MPC circuit (Huiskamp 2020).

Figure 27a shows a schematic of a MPC-type pulse power generator (Takahashi et al. 2012). After the capacitor $C_{0}$ is charged to $V_{\mathrm{C}}$, semiconductor switching device is switched-on. Since the saturable inductor $\mathrm{SI}_{0}$ has large value in inductance, current of the semiconductor switching device keeps low value during initiation phase. As the result, switching loss calculated from voltage and current in the switching device is minimized. When the capacitor $C_{1}$ is charged up to $n V_{\mathrm{C}}$, where $n$ is the amplification factor of the pulse transformer PT, the stored energy of $C_{1}$ transfers to $C_{2}$ through the saturable inductor $\mathrm{SI}_{1}$. Following the energy transfer, the energy transfer from $C_{2}$ to $C_{3}$ occurs through $\mathrm{SI}_{3}$. The energy transfer from $C_{3}$ to the load occurs in same manner. The risetime of voltage decreases gradually because of $\mathrm{SI}_{1}>\mathrm{SI}_{2}>\mathrm{SI}_{3}$ as shown in Fig. 27b

\section{High-voltage phenomena for agriculture and food processing}

Here, a basis of high-electric field phenomena related to the agricultural applications is outlined. The high electric field is usually generated as electrostatic or induction fields. The electric field induces biological effects through Maxwell force, Coulomb force, charging cell membrane up. The electric field accelerates charged particles by the Coulomb force. As a result, an electron avalanches are formed and are developed towards the electric field. The electron avalanches cause an electrical breakdown (or discharge onset) when the electric field has enough amplitude to cause the electrical breakdown. Plasma commonly includes many kinds of chemically active species which also induce biological response. In this section, the electrical field distribution in basic electrode configurations is outlined. After that, the biological effects of the electric field distribution such as electroporation, electrostimulation, and protein conformational change for controlling enzyme activity, are described. Lastly, the streamer and glow discharges generation using pulsed power is outlined as basis of pulsed power plasma production.

\subsection{Electric fields and potentials between electrodes}

The relationship between magnetic and electric fields is completely described by the set of four Maxwell's equations as.

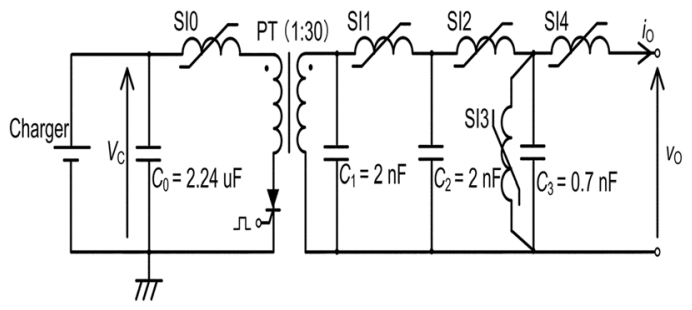

(a)

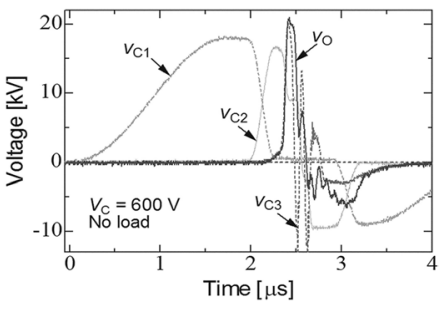

(b)

Fig. 27 Schematic of a MPC circuit and $\mathbf{b}$ its output voltage waveforms 


$$
\begin{gathered}
\nabla \times \mathbf{E}=-\frac{\partial \mathbf{B}}{\partial t}, \\
\nabla \times \mathbf{H}=\mathbf{J}+\frac{\partial \mathbf{D}}{\partial t}, \\
\nabla \cdot \mathbf{B}=0, \\
\nabla \cdot \mathbf{D}=\rho,
\end{gathered}
$$

where $\mathbf{E}$ means the electric field strength, $\mathbf{B}$ the magnetic flux density, $\mathbf{H}$ the magnetic field strength, $\mathbf{J}$ the current density, $\mathbf{D}$ the electric flux density, $r$ the charge density. If no magnetic fields are present, Eq. (13) becomes $\nabla \times \mathbf{E}=0$, which means no induction electric field. Definition of electric field is the rate at which the scalar potential $\phi$ varies with distance as follows.

$$
\mathbf{E}=-\nabla \phi
$$

The relation between electric field and electric displacement field (electric flux density) is expressed using the electrical permittivity $\varepsilon$ as follows:

$$
\mathbf{D}=\varepsilon \mathbf{E} .
$$

By substituting Eqs. (17) and (18) into (16), the well-known Poisson's equation is obtained as follows.

$$
\nabla^{2} \phi=-\frac{\rho}{\varepsilon}
$$

If no charges are present, Eq. (19) becomes Laplace's equation:

$$
\nabla^{2} \phi=0 .
$$

Laplace's equation can be used to calculate the electric fields present in different geometries. Figure 28 shows potential and electric field distributions between the electrode in three different geometries calculated using Eqs. (20) and (17), respectively. In case of an infinite parallel plate, the electric field is homogeneous in gap between the electrodes. However, in cases of a point electrode and an infinite coaxial line, the electric field strength changes with distance from the electrode with small radius of curvature.

\subsection{Biological cell exposure by electric field}

Application of electric fields to biological cells in a conducting medium causes charging up the cell membrane, and then the voltage across the membrane is changed. In case of low electric fields, this voltage change induces gating, in which the opening of channels in the cell membrane is induced. An ion flux flowing through the ion channels causes the change of ion concentrations and balances in 

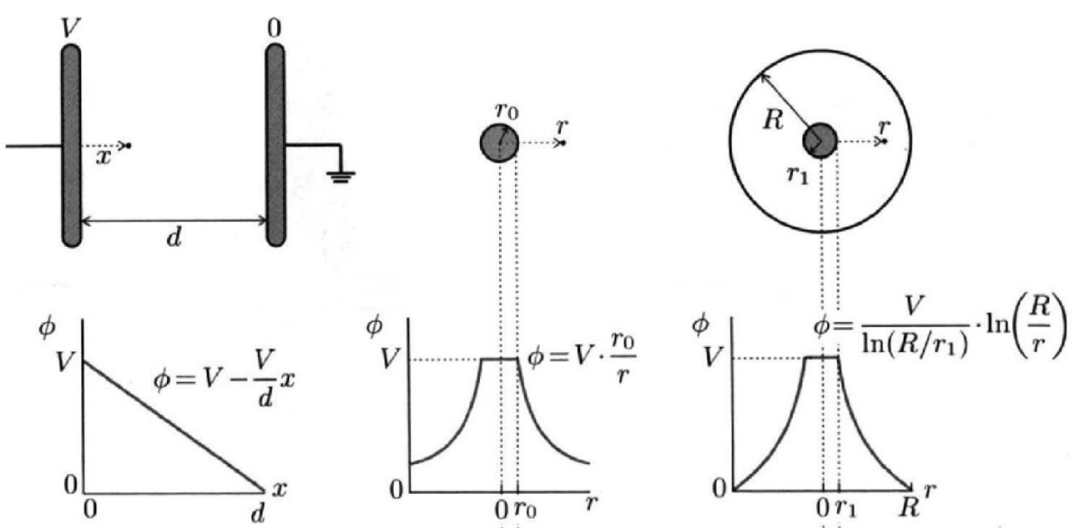

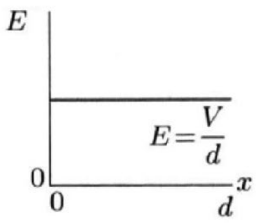

(a)

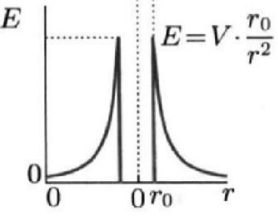

(b)

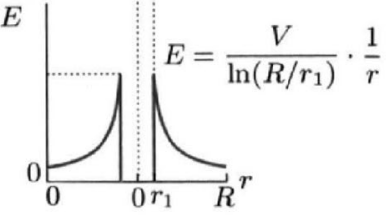

(c)

Fig. 28 Potential and electric field distributions between the electrode in three different geometries; a infinite parallel plate, $\mathbf{b}$ point electrode, and $\mathbf{c}$ infinite coaxial line

vicinity of cell membrane. This change of ion concentrations and balances works as stress of cells. The stress for short duration (on the order of milliseconds) and small electric field does not cause irreparable damage. However, the stress for long duration and high electric field causes the damage as the permeability of the membrane increases to a level that either the recovery of cell from second to hours (reversible breakdown), or cell death (irreversible breakdown) (Schoenback et al. 1997). This section outlines the phenomena of cell membrane and protein conformational change in electric field.

\subsubsection{Voltage build-up across cell membrane}

Figure 29 shows cross-sectional schematic of a biological cell and equivalent circuit using double-shell model in suspension. The equivalent circuit consists of capacitive and resistive components (Schoenback et al. 2007). The cell consists of cytoplasm, dissolved protein, electrolytes, glucose, nucleoplasm and other organelles. These components have relatively high conductivity. On the contrary, the membranes that surround the cell and subcellular structures have a low conductively. Therefore, the cell can be thought as a conductor (expressed as resistive components) surrounded by an insulating envelope which is expressed as capacitive components. These properties can be expressed as the equivalent circuit shown in Fig. 29, in which the cell membrane is described by a capacitance, $C_{\mathrm{m}}$, nuclear membrane by capacitance, $C_{\mathrm{n}}$, cytoplasm by 

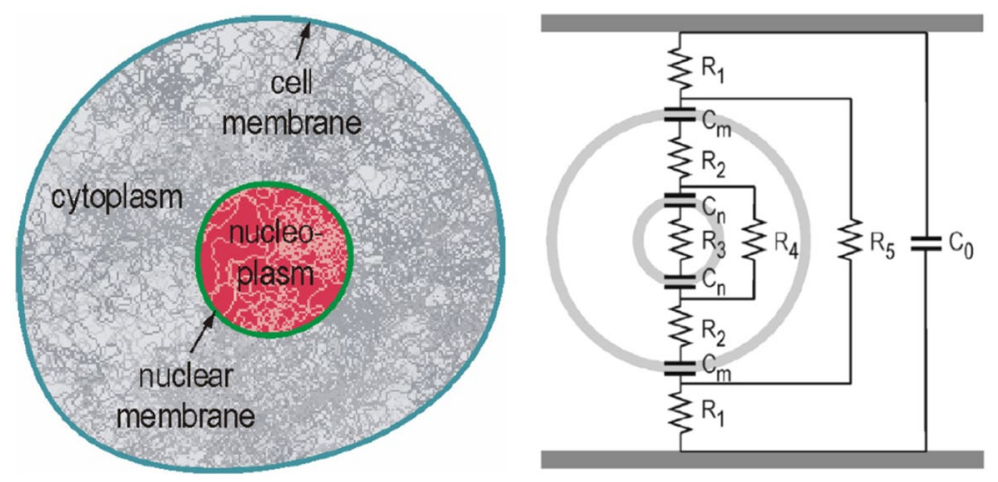

Fig. 29 Cross-section schematic of a cell with nucleus and its equivalent circuit (double shell model) in suspension (Schoenbach et al. 2007, 2008)

resistance, $R_{2}, R_{4}$, nuclearplasm by resistance, $R_{3}$, under the assumption that the conductance of the membranes are zero, and the capacitive components of cytoplasm and nuclearplasm are negligible. Usually, capacitance $C_{\mathrm{m}}$ is higher than $C_{\mathrm{n}}$. Therefore, the applicability of the model is in temporal range determined by a dielectric relaxation times of membrane and cytoplasm.

The dielectric relaxation time, $t_{\mathrm{r}}$, provides information on the impedance of resistive or capacitive component of the membrane and cytoplasm, respectively. $t_{\mathrm{r}}$ is expressed as:

$$
\tau_{\mathrm{r}}=\varepsilon / \sigma,
$$

where $e$ means the permittivity, and $s$ the conductivity. For a pulse durations, $t$, long compared to $t_{\mathrm{r}}$, the resistive component is dominant, for the short to $t_{\mathrm{r}}$, the capacitive component is dominant.

The amplitude of the critical voltage $V_{\text {crit }}$ across the membrane which affects the cell such as gating, poration, or lysing depends on the cell type and its size, and on pulse duration. The typical values of $V_{\text {crit }}$ for lysing or poration are on the order of $1 \mathrm{~V}$, for gating is approximately $100 \mathrm{mV}$ (Schoenback et al. 1997).

The corresponding electric field $E_{\text {crit }}$ in the medium (suspension) containing the cells is expressed as:

$$
E_{\text {crit }}=V_{\text {crit }} / f a,
$$

where $a$ means the cell radius and $f$ the form factor which depends on the cell shape. For example, the form factor $f$ is obtained as 1.5 for spherical cells using void theory. In case of cylindrical cells, the form factor $f$ is expressed as:

$$
f=l /\left(l-\frac{D}{3}\right)
$$

where $l$ means the cell length with hemispheres of diameter $D$ at each end. The critical field strength $E_{\text {crit }}$ of bacteria with dimensions of $1 \mathrm{~mm}$ is estimated as order of 
$10 \mathrm{kV} / \mathrm{cm}$ for lysing by critical voltage $V_{\text {crit }}$ of $1 \mathrm{~V}$ for pulse of tens of microsecond to millisecond duration. Microorganisms other than bacteria have dimensions in the range of 10-40 $\mathrm{mm}$. Therefore, these microorganisms are much vulnerable to electric field compared with bacteria (Schoenback et al. 1997).

When a squared wave pulse with voltage of $V_{\mathrm{a}}=E \times d$ is applied, where $d$ is the distance between electrodes and the $E$ electric field in medium, the voltage across the membrane $v_{\mathrm{m}}$ at the poles increases with time $t$ as follows:

$$
v_{m}(t)=f E(D / 2)\left[1-e^{-t / \tau_{c}}\right]+v_{0},
$$

where $v_{0}$ means the resting voltage. The resting voltage $v_{0}$ is approximetely $70 \mathrm{mV}$ for many cells. The time constant for charging the membrane, $t_{\mathrm{c}}$, is expressed as:

$$
\tau_{\mathrm{c}}=\left[\left(\frac{1+2 V}{1-V}\right) \frac{\rho_{1}}{2}+\rho_{2}\right] C_{\mathrm{m}} a,
$$

where $r_{1}$ and $r_{2}$ are resistivity of the suspending medium and cytoplasm, respectively, $C_{\mathrm{m}}$ the membrane capacitance per unit area, and $V$ the spherical cells volume. For a mammalian cell, the time constant for charging cell membrane can be estimated as $75 \mathrm{~nm}$ using $10 \mathrm{~mm}$ diameter, resistivities of $100 \mathrm{Wcm}$, and a volume concentration small compared to one (typical in vitro experimental condition) (Schoenback et al. 1997).

\subsubsection{Critical field for microorganism survivability}

Survivability, $s$, which is defined as the fraction of surviving microorganisms, decreases exponentially with increasing amplitude of electric field and linearly with increasing exposure time. The survivability $s$ is expressed using empirical law for pulse of $>50 \mathrm{~ms}$ duration and $>8 \mathrm{kV} / \mathrm{cm}$ amplitude as follows:

$$
s=\left(\frac{\tau}{\tau_{0}}\right)\left(\frac{E-E_{\text {crit }}}{E_{0}}\right),
$$

where $t$ is pulsed duration, $E$ the strength of the applied field, $E_{\text {crit }}$ the threshold field below which no effect is observed, $t_{0}$ and $E_{0}$ are constants which depend on the type and size of cell, and suspending medium, respectively. For example, Hülsheger obtained the electrical parameters with analysis of the measured survivability as shown in Fig. 30 by best fitting with $E_{\text {crit }}=4.9 \mathrm{kV} / \mathrm{cm}, E_{0}=6.3 \mathrm{kV} / \mathrm{cm}$, and $t_{0}=12 \mathrm{~ms}$ (Hülsheger et al. 1981). From this empirical law, the required electrical energy density for lysing $W$, is expressed as:

$$
W=\tau \sigma E^{2},
$$

where $E$ is the electric field strength $E, s$ the conductivity, and $t$ the pulse width decreases with reducing pulse width $t$ in the pulsed duration in range of $>50 \mathrm{~ms}$. The Eqs. (26) and (27) indicate that the high electric field and short pulse process serves to improve the efficiency of the process. The Eq. (27) also means that for long 
Fig. 30 Survival rate of $E$. coli as function of electric field exposer time for various field strengths (Hülsheger et al. 1981)

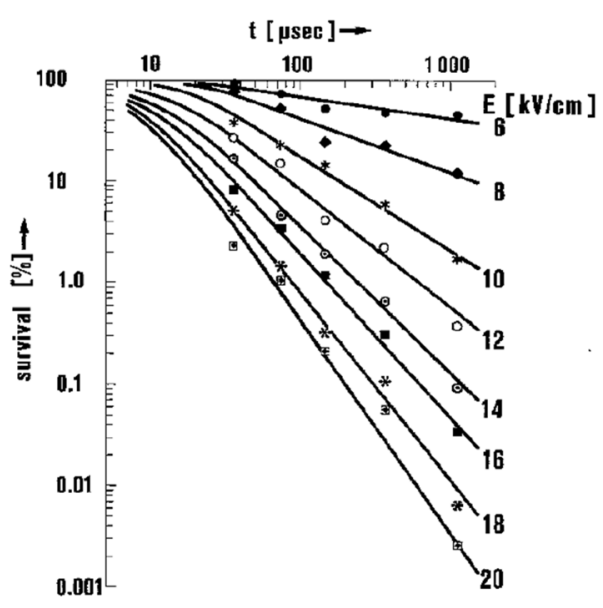

pulses thermal effects begin to play role. For example, a $1 \mathrm{~ms}$ pulse with a voltage of $0.5 \mathrm{~V}$ across one membrane would lead to a temperature increase more than 20 degrees in assumption of adiabatic process. Therefore, the thermal effects also affect the process for long width pulse.

The characteristics parameters $E_{\text {crit }}, E_{0}$, and $t_{0}$ for short-duration pulse of $<5 \mathrm{~ms}$, however, differ from those for long-duration pulse of $>50 \mathrm{~ms}$. For example, Schoenback obtained with pulses of less than $2 \mathrm{~ms}$ duration, $E_{\text {crit }}$ is changed to $40 \mathrm{kV} / \mathrm{cm}, E_{0}$ and $t_{0}$ are changed to $80 \mathrm{kV} / \mathrm{cm}$ and to $10 \mathrm{~ns}$, respectively. The value of the electric field required for one order reduction of the $E$. coli in tap water is $164 \mathrm{kV} / \mathrm{cm}$ for $60 \mathrm{~ns}$ pulses, $107 \mathrm{kV} / \mathrm{cm}$ for $300 \mathrm{~ns}$ pulses, and $66 \mathrm{kV} / \mathrm{cm}$ for $2 \mathrm{~ms}$ pulses, as shown in Fig. 31. The energies required for one order reduction of the $E$. coli are $0.85 \mathrm{~J} / \mathrm{cm}^{3}$ for $300 \mathrm{~ns}$ pulses, $1.8 \mathrm{~J} / \mathrm{cm}^{3}$ for $300 \mathrm{~ns}$ pulses, and $4.6 \mathrm{~J} / \mathrm{cm}^{3}$ for $2 \mathrm{~ms}$ pulses (Schoenback et al. 1997). Assuming

Fig. 31 Effect of electric field pulses on the survivability of $E$. coli in tap water for 60,300 and $2 \mu$ s pulse widths (Schoenbach et al. 1997)

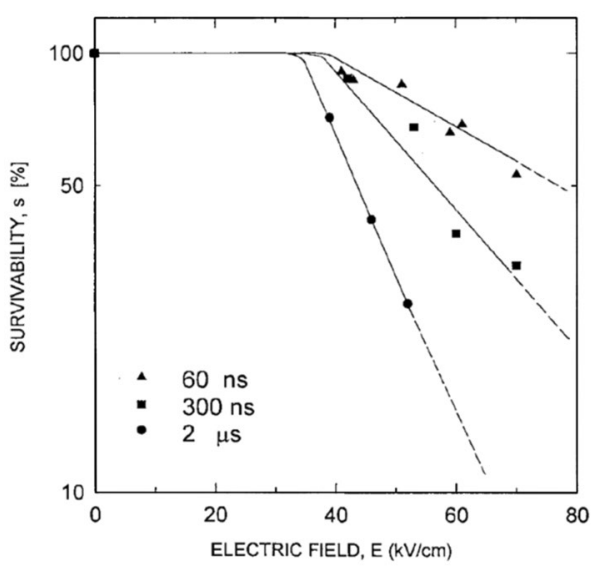


that the biological processes caused by intense of short pulse, the threshold of electric field intensity $E_{\text {crit }}$ is given as:

$$
E>E_{\text {crit }}=\frac{V_{\text {crit }}(2 / f D)}{1-e^{\left(-t / \tau_{\mathrm{c}}\right)}} .
$$

This equation can be written as:

$$
E \tau>(E \tau)_{\text {crit }}=V_{\text {crit }}\left(2 \tau_{c} / f D\right) .
$$

Using the equation, the critical applying electric field can be estimated as $13.3 \mathrm{kV} / \mathrm{cm}$ at a critical transmembrane voltage of $1 \mathrm{~V}$, a spherical cell with a diameter of $10 \mu \mathrm{m}$, and a time constant of $100 \mathrm{~ns}$ for charging membrane (Schoenback et al. 2007).

\subsubsection{Effect on subcellular organelles}

Pulse electric field with short duration lower (nsPEFs) than 100 ns (typical charging time for membrane) induces biological effects in intracellular with the intense electric field inside the cell adding a stress to the biological system (Akiyama and Heller 2017; Schoenback et al. 2007; Katsuki et al. 2007). The intracellular effects of the nsPEFs are a result of the high-frequency components exceeding the $\mathrm{MHz}$ range which passes through cell membrane (capacitive components) and directly stimulating intracellular organelles including DNA.

Nomura reported that the calculating results of electric field strengths of membranes, cytoplasm and nucleus using a model as shown in Fig. 32 (Nonomura et al. 2009). The calculating conditions are a $10 \mu \mathrm{m}$ diameter simplified spherical cell suspended in a conductive solution between parallel electrodes. The gap length between the electrode and applied voltage are $20 \mu \mathrm{m}$ and $2 \mathrm{~V}$, respectively, then an average electric field is obtained as $100 \mathrm{kV} / \mathrm{m}$. The spherical cell consists of plasma membrane, cytoplasm, nuclear membrane and nucleoplasm. The electric field strengths
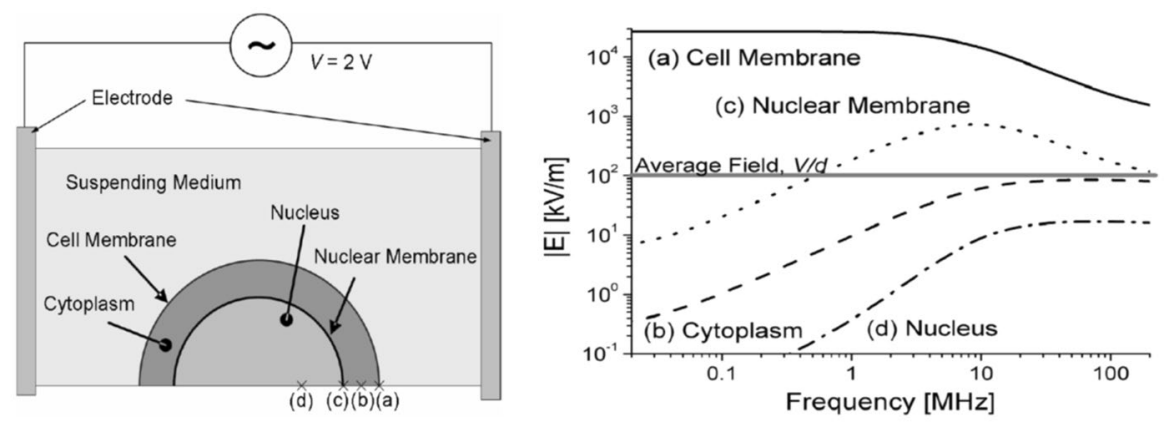

Fig. 32 Schematic of the simplified cell model and calculated electric field strengths at the positions a-d as functions of the frequency at average electric field of $100 \mathrm{kV} / \mathrm{cm}$ (Nonomura et al. 2009) 
at the plasma membrane, cytoplasm, nuclear membrane and nucleoplasm are calculated for various applied voltage frequencies. The calculation results indicates that the almost electric field appears at the cell membrane because the conduction current is interrupted with the capacitive components for frequencies below $3 \mathrm{MHz}$. The field strength at the membrane is almost constant at approximately $25 \mathrm{MV} / \mathrm{m}$, which corresponds to a field enhancement ratio of 250 . This field strength is comparable to the level $(100 \mathrm{MV} / \mathrm{m})$ to form pores on the cell membrane $(100 \mathrm{MV} / \mathrm{m})$. The electric field at the membrane decreases with increasing frequency above $3 \mathrm{MV}$, in contrary, the electric field starts to penetrate into the cell. The electric fields at the cytoplasm and nuclear membrane increase to the same range of external field in the frequency larger than $10 \mathrm{MHz}$.

Figure 33 shows intracellular DNA damages caused by intense burst sinusoidal electric fields as a function of frequency and strength of applied electric field using Chinese hamster ovary cells exposure by $200 \mathrm{~ms}$ long burst pulse (Nomura et al. 2007). The intracellular DNA damages are monitored by an alkaline comet assay method. The results show that $100 \mathrm{kV} / \mathrm{m}$ electric field with frequencies exceeding $1 \mathrm{MHz}$ or $100 \mathrm{MHz}$ electric field with field strengths exceeding $3 \mathrm{kV} / \mathrm{m}$ induces significant DNA damage. The minimum field strength which causes the DNA damage is $10 \mathrm{kV} / \mathrm{m}$. This intracellular strong field works as stress for triggering biological processes leading to the DNA damage (Akiyama and Heller 2017; Schoenback et al. 2007; Katsuki et al. 2007).

\subsection{Protein exposure by electric field}

Intense pulse electric fields (PEFs) are tried to be used in food processing such as inactivation of bacteria, enzyme activity control, and fermentation accelerations as non-thermal processing which can reduce an influence on food quality. The advantages of PEFs treatment compared to a thermal process are reducing detrimental changes of nutrition, retaining the physical and sensorial qualities of the food (Syed et al. 2017). PEF treatment is applied to wide range foods such as liquid (juice, milk, beer, etc.), semi-solid (gel state foods), and solid-state foods. Typical operation
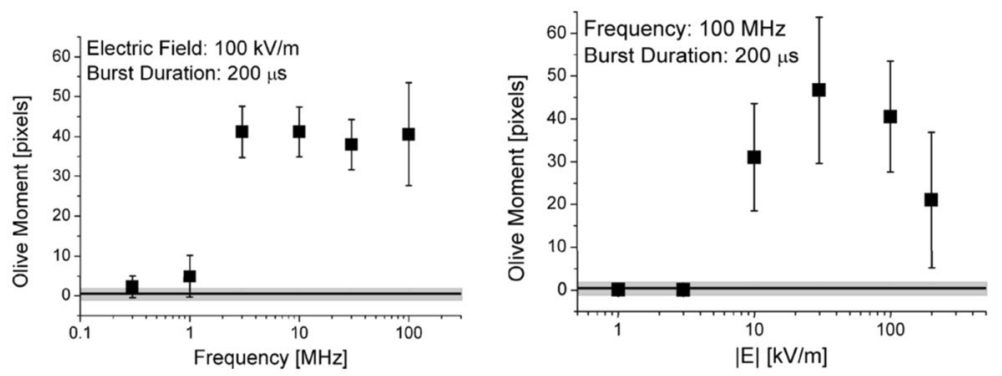

Fig. 33 Apoptosis induction monitored by olive moment as a function of frequency and strength of the applied electric field (Nomura et al. 2007) 
range of PEF treatments is an electric field amplitude of 5-50 kV/cm with pulse length of in range several to tens- $\mu$ s. PEFs are applied to the fool located between two electrodes and causes bacteria and enzymatic inactivation at a temperature lower than thermal treatment (Mohamed and Eissa 2012; Ohshima et al. 2007). The enzymatic reactions are basically function on protein, which are determined by its conformation of the polypeptide chain. Therefore, the enzyme is inactivated by the protein conformational change such as misfolding of proteins. The conformational stability of the protein is important for activity of enzyme. The conformation of protein consists of secondary structures (such as $\alpha$-helix and $\beta$-sheet) and tertiary structure. The protein exposure by intense PEFs causes a conformational change through processes of electrical charging up and displacement of elements by electrical force (Bekard and Dunstan 2014).

\subsubsection{Conformational change through direct stretch}

Protein exposure by intense electric field causes conformational structure change through direct or indirect affecting the secondary and tertiary structures. The direct effect of intense PEFs is stretching the molecular bindings in protein, i.e., an unfolding structure caused by an electrostatic tensile force. Jiang et al. reported that the secondary structure was predicted to change from helix to turn or random coil at electric field with strength $E>0.5 \mathrm{~V} / \mathrm{nm}\left(5.0 \times 10^{8} \mathrm{~V} / \mathrm{m}\right)$ using molecular dynamics (MD) calculations, as shown in Fig. 34 (Jiang et al. 2019). The MD calculations are carried out for the 1BBL (consisting of 37-amino acid residues) protein molecule including two $\alpha$-helix secondary fragments. The 1BBL protein original structure still remain in case without exposure by electric field for all orientations during the period set in calculation. However, the protein is stretched for all orientations with exposure by electric field with relatively high strength. This result indicates that the

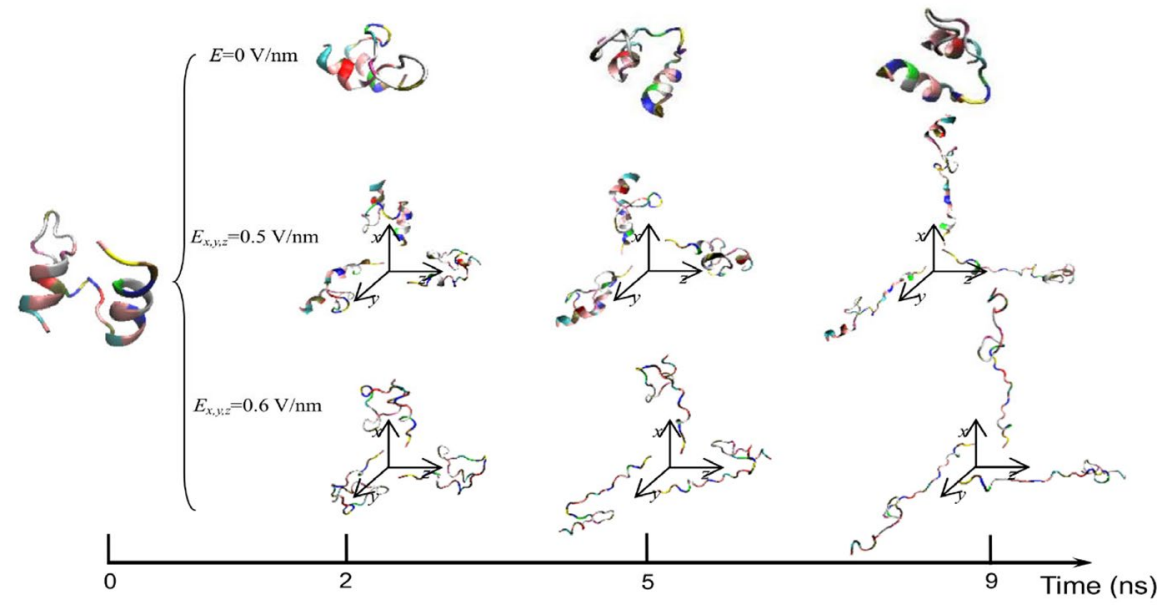

Fig. 34 Typical conformations of 1BBL protein exposed by intense electric fields $E x, y, z=0,0.5$, and $0.6 \mathrm{~V} / \mathrm{nm}$ (Jiang et al. 2019) 


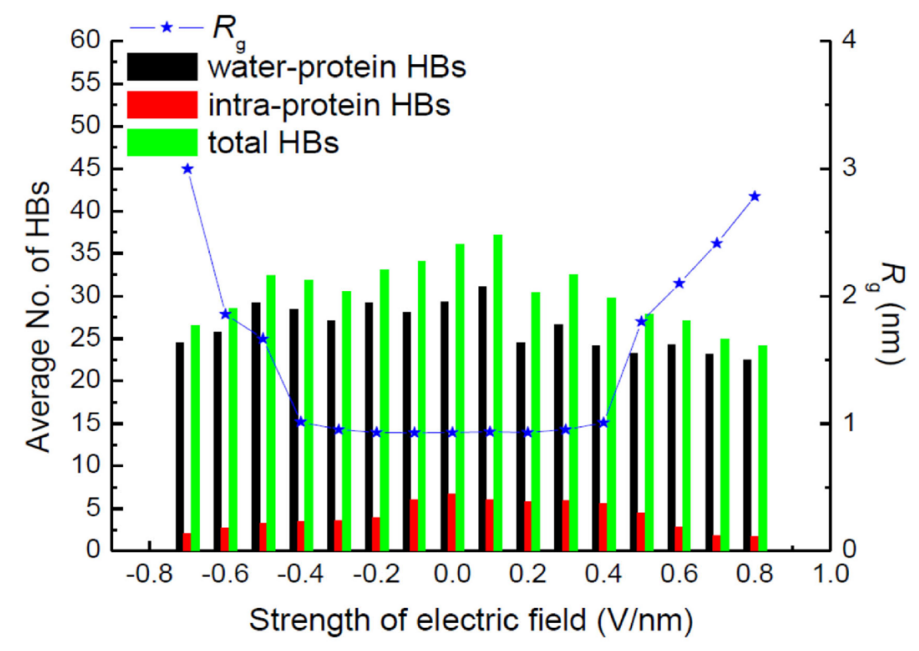

Fig. 35 Average number of hydrogen bonds and radius of gyration $R_{\mathrm{g}}$ of the protein $1 \mathrm{BBL}$ exposed in the electric fields along the $z$-direction with different strengths (Jiang et al. 2019)

realignment of some charged residues is induced by the electric field exposing. The structure change from the $\alpha$-helix to the turns and random coils causes more rapid by increasing electric field strength.

Hydrogen bonds (HBs) are important in role for stabilizing the conformation such as secondary structure. The average number of total HBs in protein structure is calculated using MD simulation at various strengths of an exposed electric field, as shown in Fig. 35 (Jiang et al. 2019). The average number of intra-protein HBs decreases with an increasing electric field strength larger than $0.5 \mathrm{~V} / \mathrm{nm}$. The number of intra-protein HBs has a strong relationship with the conformational structure stability of the protein. Moreover, the radius of gyration $R_{\mathrm{g}}$ has the opposite tendency, against that of the number of intra-protein HBs.

Qin and Buehler reported that the protein secondary structural transitions depended on amino acid chain length. The short amino chain proteins with fewer than 26 amino acids (i.e., $3.8 \mathrm{~nm}$ length) is easily induced as interprotein sliding. However, the long amino chain proteins with larger length causes a conformational change from $\alpha$-helix to $\beta$-sheet, which lead to increase the protein stiffness, strength, and energy dissipation capacity (Qin and Buehler 2010; Marracino et al. 2013).

Valle et al. reported MD analysis of the conformational change of a single superoxide dismutase (SOD-1) enzyme by exposing $100 \mathrm{~ns}$ width intense PEF in range from $10^{8}$ to $7 \times 10^{8} \mathrm{~V} / \mathrm{m}$ in strength. The analysis results indicate that the electric pulses above $5 \times 10^{8} \mathrm{~V} / \mathrm{m}$ (threshold intensity) induce a fast transition between the folded and a partially denatured state, as inferred by the secondary structure analysis as shown in Fig. 36 (Valle et al. 2019). In the MD calculations, a monopolar (MP) or a bipolar (BP) of $100 \mathrm{~ns}$ PEF is applied to SOD-1. The intensity of $7 \times 10^{8} \mathrm{~V} / \mathrm{m}$ induces a dramatic structure change with an irreversible transition from $\beta$-sheet or coil structures to an unfolded states (Sheu et al. 2003). 
(a) No-field

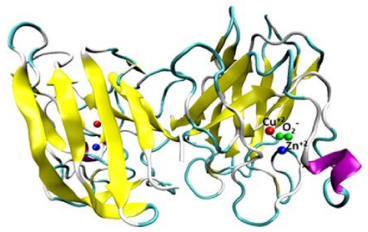

(b) Pulsed

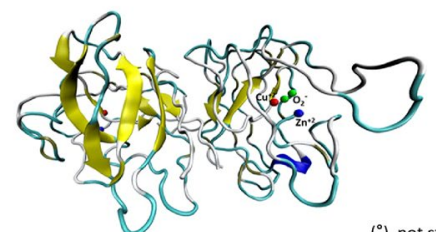

(c)

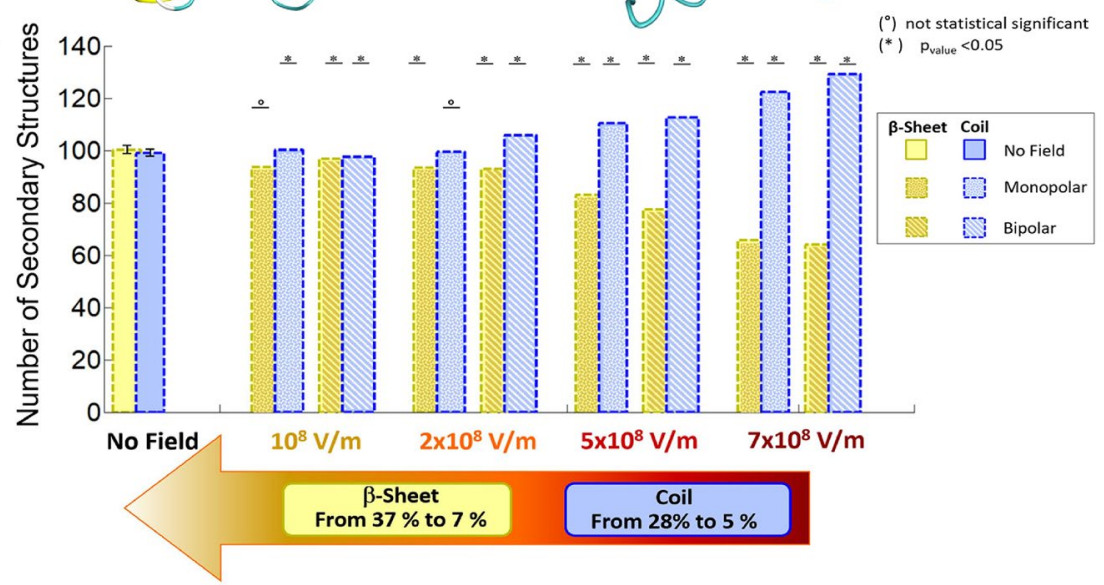

Fig. 36 Comparison of the conformation of SOD1 before (a) and after an exposure to electric field of $7 \times 10^{8} \mathrm{~V} / \mathrm{m}$ strength (b). Number of coil and $\beta$-sheet secondary structures (c) (Valle et al. 2019)

Ding et al. calculated the electric force on the proteins which induces the conformational change with applied forces relative to the inter-chain bonding forces. The inter-chain bonding of HBs in the $\alpha$-helix and $\beta$-sheet are $8.1 \mathrm{~kJ} / \mathrm{mol}(1.93 \mathrm{kcal} /$ $\mathrm{mol})$ and $6.6 \mathrm{~kJ} / \mathrm{mol}(1.58 \mathrm{kcal} / \mathrm{mol})$, respectively. Using the bonding energies of HBs and a distance between the elements of $0.35 \mathrm{~nm}$, the inter-chain bonding forces of $\mathrm{HB}$ is obtained as $40 \mathrm{pN}$, which corresponds to approximately $10^{8} \mathrm{~V} / \mathrm{m}$ in electric field strength (Ding et al. 2003). The transition in conformational structure from $\alpha$-helix to $\beta$-structure was also analyzed based on the four-bead model using discrete MD modeling. The potential energy $(\varepsilon \mathrm{HB})$ of $\beta$-hairpin structure is larger than that of $\alpha$-helix. However, the entropy of a $\beta$-hairpin is larger than that of $\alpha$-helix. From the free energy of the HB for $\alpha$-helix and $\beta$-hairpin conformations, the $\alpha$-helix to $\beta$-hairpin transition is predicted to be caused at $0.125 \varepsilon \mathrm{HB}$ of the temperature (Ding et al. 2003). In here, the connection of primary structure consists of covalent bonds such as peptide bond and disulfide (S-S) bond. These bonds have almost one order higher bonding energy $(210-630 \mathrm{~kJ} / \mathrm{mol})$. For this reason, the primary structure is generally less sensitive to electric fields compared with secondary and tertiary structures.

\subsubsection{Conformational change by slow process}

Conformational changes of protein were also confirmed in relatively low electric field strength $(<0.5 \mathrm{~V} / \mathrm{nm})$ and long-time exposure. Bekard and Dunstan reported 


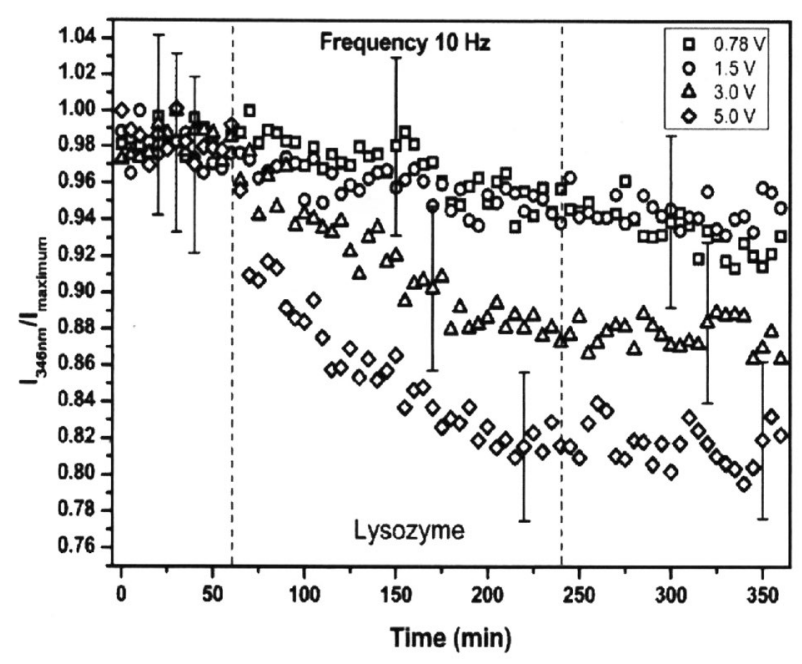

Fig. 37 Time-evolution of the relative emission intensity of Lysozyme solutions at $0.2 \mathrm{mg} / \mathrm{mL}(\mathrm{pH} 7.2)$ monitored at $346 \mathrm{~nm}$ for exposure to varying electric field strengths. The electric field strengths are ( $\square$ ) $0.78,(\bigcirc) 1.5,(\Delta) 3.0$ and $(\diamond) 5.0 \mathrm{~V} / \mathrm{cm}$. The dotted lines indicate partitioning into the first $1 \mathrm{~h}$ without electric field, followed by $3 \mathrm{~h}$ of electric field exposure, and a further $2 \mathrm{~h}$ of without electric field (Bekard and Dunstan 2014)

that conformational change lysozyme in $\mathrm{AC}$ low electric field of $10 \mathrm{~Hz}$ in frequency with range from 0.78 to $5.0 \mathrm{~V} / \mathrm{cm}$, as shown in Fig. 37 (Bekard and Dunstan 2014). The conformational changes are monitored with time evolution of the relative emission intensity of lysozyme solutions at $346 \mathrm{~nm}$ of the tryptophan fluorescence emission with excitation wavelength of $295 \mathrm{~nm}$ wavelength. The conformation during first $1 \mathrm{~h}$ monitors without exposing AC electric field, shown by dotted vertical lines, followed by $3 \mathrm{~h}$ with AC electric field exposure, and a further $2 \mathrm{~h}$ without the electric field again. In the experiment, it was confirmed that the tryptophan fluorescence emission not only decreased its intensity, but red shift of the emission wavelength peak was caused by exposing electric field (Bekard and Dunstan 2014). The spectral changes generally indicate alterations in the microenvironment of tryptophan residues, and typically reflect the exposure of these residues, initially concealed in hydrophobic segments of the folded protein, to the surrounding aqueous environment. The decrements of relative fluorescence emission intensity of lysozyme are observed for all exposure electric field strengths, and is more pronounced at field strength up to $5.0 \mathrm{~V} / \mathrm{cm}$. The decrease in tryptophan emission intensity appeared irreversible. Further analysis of the data indicates a linear relation between the relative tryptophan emission intensity and the applied electric field strength, as shown in Fig. 38 (Bekard and Dunstan 2014).

Bekard and Dunstan also reported that the fractions of secondary structure of lysozyme solutions were changed from $31 \% \alpha$-helix, 20\% b-strands, $20 \%$ b-turns and $29 \%$ random coil to $19 \% \alpha$-helix, $28 \%$ b-strands, $23 \%$ b-turns and $30 \%$ random coil by $3-\mathrm{h}$ exposure with electric field of $3.0 \mathrm{~V} / \mathrm{cm}$ strength. The electric field strength 


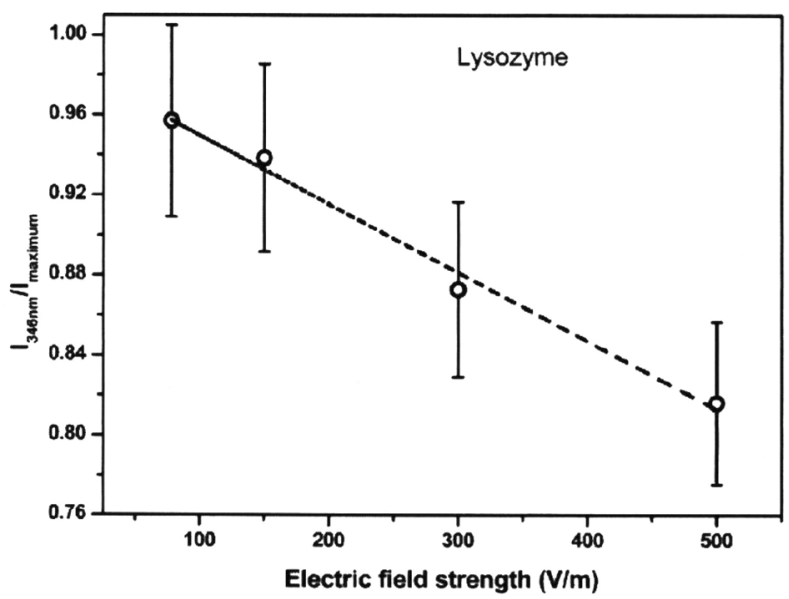

Fig. 38 Relative fluorescence emission intensity of Lysozyme solutions $(0.2 \mathrm{mg} / \mathrm{mL}, \mathrm{pH} 7.2)$ monitored at $346 \mathrm{~nm}$ as a function of electric field strength after $3 \mathrm{~h}$ of electric field exposure. The dotted line is linear fit with $R^{2}$ of 0.99 (Bekard and Dunstan 2014)

of $3.0 \mathrm{~V} / \mathrm{cm}$ corresponds to be $0.1 \mathrm{fN}$ of electrical force on $\mathrm{HBs}$ in the protein, which is almost six orders of magnitude lower less than the HB bonding forces. To solve the inconsistency, Bekard and Dunstan proposed the model of indirect effect (slow process), which is based on the electrophoretic motion (electrostatic interactions) of protein leading a frictional force for the protein unfolding (Bekard and Dunstan 2014). The electrostatic effect is basically caused with the oppositely charged terminal residues, charged side chains, and peptide dipoles in the secondary structure segments of protein. The dipole moment of lysozyme, which has net charge of +7 , is roughly calculated as 74 Debye length at natural $\mathrm{pH}$. The alignment of secondary structure dipoles strongly affects in stability of the tertiary structure of proteins. In addition, the macro-dipole can distort the field distribution and produce relatively strong local electric fields. The electric field strength along a helix axis is estimated to be in the region of $10^{9} \mathrm{~V} / \mathrm{m}$.

Furthermore, the electric dipole of lysozyme induces periodic realignment in the direction of the field resulting in a torque induced by exposure to an external oscillating (AC) electric field. However, the motion of the protein by the torque in the electric field is hampered by a drag force caused by hydrodynamic friction. If the protein configurations are assumed as spherical, the frictional energy $E_{\text {friction }}$ can be calculated using the Stokes drag on the protein as follows:

$$
E_{\text {friction }}=\int_{0}^{x} f_{\text {drag }} \mathrm{d} x=\int_{0}^{x} 6 \pi \eta a v \mathrm{~d} x=6 \pi \eta a v U_{m}{ }^{2} E^{2} t,
$$

where $f_{\text {drag }}$ means the Stokes drag force, $x$ the electrophoretic movement distance, $q$ the charge on the protein, $\eta$ the solvent viscosity, $a$ the protein radius, $v$ the electrophoretic velocity, $E$ the electric field strength, $U_{\mathrm{m}}$ the mobility. The friction energy $E_{\text {friction }}$ is calculated to be $1.7 \times 10^{5} \mathrm{~kJ} / \mathrm{mol}$ for lysozyme at $5.0 \mathrm{~V} / \mathrm{cm}$ field strength 
and 200 min exposure time. This frictional energy is corresponding three orders higher energy of hydrogen bonding energy. However, only a small percentage of the frictional energy is used to disrupt the HBs in protein. Therefore, the long exposure time is necessary for the conformational change from $\alpha$-helix to b-sheet through the electrophoretic motion (Bekard and Dunstan 2014).

\subsection{Atmospheric plasmas for agriculture and food processing}

Electrical discharges are used to create plasma as source of ion and chemically active species such as radical oxygen species and radical nitrogen species (Graves 2012; Reuter et al. 2018). The electrical discharges can be initiated in localized regions of high field strength, then continue to propagate into regions where the field is not strong enough to start a discharge, but it is strong enough to allow the discharge to continue to propagate. Whether the discharge is transient or continuous can also depend on how the discharge gets its power.

Electrical discharges are fundamentally ionizing materials (gases, liquids, or solids) to produce charge carriers (electrons and ions). There is always a low level of background ionization occurring due to background radiation, which comes mainly from natural sources such as radon gas and cosmic rays. The most important ionization mechanism in electrical discharges is electron impact ionization. Free electrons impact on the outer electron orbitals of atoms and molecules all the time. If the free electrons have enough energy, they can knock the outer electron out of its orbital, leaving a positive ion and another free electron. The free electrons get their energy from externally applied fields. The field applied to create a discharge is generally the electric field; however, a magnetic field is also used to create inductively coupled discharges (Braithwaite 2000).

\subsubsection{Electron multiplication: avalanche process}

When the electric field between the electrodes is high enough to accelerate the electrons, the accelerated electrons ionize the neutral atoms and molecules, producing more electrons. These produced electrons are also accelerated by the intense electric field to ionize even more atoms, producing even more free electrons. This contentious ionization process is called as an avalanche build-up process. This is the moment of inception of a high-voltage breakdown, and if the conditions are right, it can cause complete flashover of the electrodes (Kuffel et al. 2000).

The avalanche process was first mathematically described by Townsend in 1897 . Consider an avalanche discharge between two electrodes. The position between the electrodes is given by the variable $x$, which defined as distance from negative (or grounded) electrode, as shown in Fig. 39a. The differential number density of electrons $\mathrm{d} n_{x}$ multiplied in a distance $\mathrm{d} x$ depends on the number of electrons at that point $n_{x}$ and the Townsend's (primary) ionization coefficient $\alpha$ :

$$
\mathrm{d} n_{x}=n_{x} \alpha \mathrm{d} x .
$$


Fig. 39 Schematics of electron multiplication

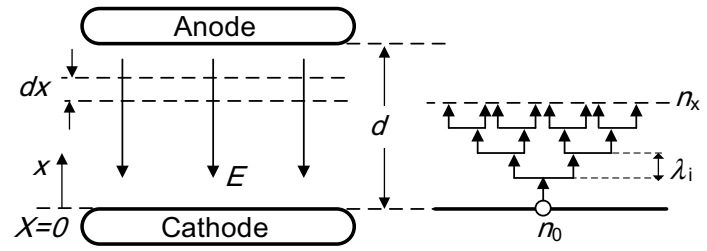

(a)

(b)

The primary ionization coefficient $\alpha$ is defined as number of electrons produced per unit length. By integration and the fact that $n_{\mathrm{x}}=n_{0}$ at $x=0$, this gives.

$$
n_{x}=n_{0} e^{\alpha x}
$$

The number of free electrons (and ions) increases exponentially in the avalanche.

The avalanche growth is expressed as increase of electron, as shown in the Fig. $38 \mathrm{~b}$, as $n_{x}=n_{0} e^{k}$, where $k$ is the number of ionizing steps $\left(k=x / l_{\mathrm{i}}\right.$; $l_{\mathrm{i}}$ the ionization mean free path). The relation between the ionization mean free path $l_{\mathrm{i}}$ and the total scattering mean free path $l$ was obtained by Townsend as a process of the drift energy gained from the electric field $\mathrm{El}$, with an ionization energy $e V_{\mathrm{i}}$, where $V_{\mathrm{i}}$ means ionization potential as follows (Braithwaite 2000).

$$
\alpha=\frac{1}{\lambda_{i}}=\frac{\text { constant }}{\lambda} \exp \left(\frac{-V_{i}}{E \lambda}\right) .
$$

The coefficient can be written as follows using gas pressure $p$, because the mean free path is inversely proportional to the gas pressure,

$$
\alpha=A p \cdot \exp \left(\frac{-B p}{E}\right)
$$

where the constants $A$ and $B$ are constants determined by the gas properties.

\subsubsection{Transition to self-sustained discharge: Townsend mechanism}

Townsend also considered secondary ionization processes. In addition to electron impact ionization process, secondary electron emission process is also needed to include. The secondary electrons are emitted from a cathode by impact of positive ions which are drifted with external electric field at a rate of $\gamma$ electrons per indicate ion ( $\gamma$ : secondary ionization coefficient). The processes of secondary emission and electron multiplication induces self-sustaining feedback process if the ions impact secondary electron emission is sufficient to replenish number of electrons into the gap. From the Eq. (32), $n_{0}$ initial electrons will be estimated to produce $\left(n_{0} \mathrm{e}^{a x}-n_{0}\right)$ ions at position $x$. Therefore, total number of produced ions is obtained as $n_{0}$ $\left(\mathrm{e}^{a d}-1\right)$ when the electrons moved across the gap. The criterion of self-sustaining the discharge is expressed as follows, 


$$
\gamma n_{0}\left(e^{\alpha d}-1\right)=n_{0}
$$

or.

$$
\alpha d=\ln \left(1+\frac{1}{\gamma}\right) .
$$

In general, Townsend breakdown (self-sustaining discharge onset) criterion of ad value is obtained as 8-10 (Kuffel et al. 2000).

\subsubsection{Streamer mechanism of spark}

Figure 40a shows the distribution of particles in a single avalanche. The discharge has a negative head, comprising the avalanche of free electrons, and a positive tail, comprising the positive ions left behind after the ionization avalanche has passed. The ions are at least 1800 times heavier than the electrons, so they take much longer to accelerate than the electrons. Compared to the fast free electrons, the ions only move a tiny distance from where they were born.

The difference in mobility of the ions and electrons creates the charge distribution, which in turn produces the electric field. If there are no space charges, the electric field between the electrodes is constant and is simply expressed as $V / d$ in case of parallel plane electrodes. Even though the total amount of positive ions and electrons is approximately equal, the distribution of charges in the avalanche causes an increase in the field in front of and behind the avalanche. The avalanche emits photons that can ionize nearby atoms creating free electrons. Any free electrons created in the higher-field region in front of or behind the initial avalanche will go on to produce additional avalanches.

Each additional avalanche creates more photons and further enhances the electric field. In this way a chain of avalanches propagates from the head and tail of the initial avalanche. The name for this chain of avalanches is a streamer. Streamers propagate in both directions, as shown in Fig. 39b. Eventually, the chain of

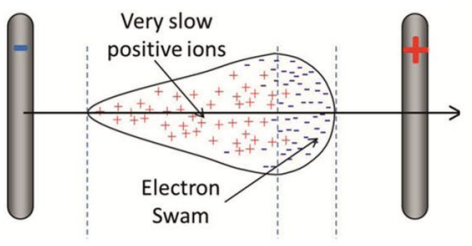

(a)

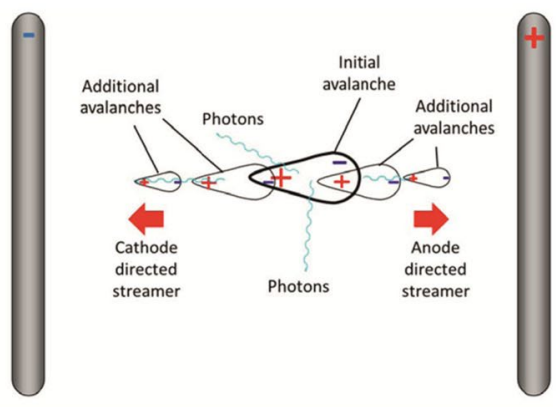

(b)

Fig. 40 Schematics of a distribution of particles in a single avalanche discharge, $\mathbf{b}$ streamer formation made up of a series of avalanches propagating in both directions from the initial avalanche 
Fig. 41 Electric field by space charge $E_{\mathrm{r}}$ around avalanche head

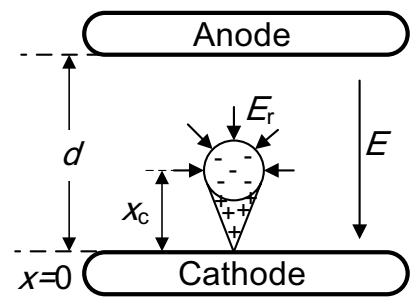

avalanches bridges the gap between the electrodes, which process creates an ionized conductive channel, following that, the gap flashes over.

Based on an experimental observation and some simple assumptions, the streamer spark criterion is expressed by Raether as an empirical expression as follows,

$$
\alpha x_{\mathrm{c}}=17.7+\ln x_{\mathrm{c}}+\ln \frac{E_{\mathrm{r}}}{E},
$$

where $E_{\mathrm{r}}$ means the strength of d produced by space charge at the head of avalanche (in Fig. 41), $E$ the external electric field (Kuffel et al. 2000). The total strength of electric field in front of the avalanche is $\left(E+E_{\mathrm{r}}\right)$, in contrary, the strength of electric field is reduced to $\left(E-E_{\mathrm{r}}\right)$ in the positive ion region (i.e., just behind the avalanche head). The Eq. (37) also shows that the number of electrons increases with the avalanche propagation length $x$ as $e^{a x}$.

The criterion of transition from avalanche to streamer is roughly expressed as that the strength of space charge field $E_{\mathrm{r}}$ is almost equal to the external field $\left(E_{\mathrm{r}} \fallingdotseq E\right)$. Therefore, the streamer breakdown criterion (37) can be simplified as:

$$
\alpha x_{\mathrm{c}}=17.7+\ln x_{\mathrm{c}} .
$$

The breakdown criterion for a uniform field gap (parallel plane electrodes) by streamer mechanism is obtained on the assumption that the transition from avalanche to streamer occurs when the avalanche has just propagated the gap $d$.

$$
\alpha d=17.7+\ln d .
$$

The streamer criterion for breakdown is roughly obtained as $\alpha d=18-20\left(\ln 10^{8} \cong 18.5\right)$, with $x_{\mathrm{c}} \leq d$. The Townsend criterion for breakdown is satisfied the $\alpha d=\ln (1+1 / \gamma)=8-10$ as mentioned in Sect. 4.4.2. The transition of Townsend to streamer breakdowns is caused by increased gas pressure $p$ and gap length $d$. In practice, it occurs in region of $p d \geq 1-2 \mathrm{bar} \mathrm{cm}$.

\subsubsection{Non-thermal plasmas for applications}

The agricultural and food processing applications of pulsed power through an nonthermal plasmas are mainly through biochemical reactions by chemically active species produced by the plasmas (Graves 2012; Reuter et al. 2018). In general, agricultural and food processing applications require the operation at atmospheric 
pressure gas or in liquid. Atmospheric pressure plasmas are basically generated through ionization process by electron acceleration with intense electric field, including production of excited species and ions. Although the input energy consumed in heating up the gas and charged particles in thermal plasmas, most of the input energy contributes to production of energetic electrons in non-thermal plasmas, i.e., cold plasmas (Tendero et al. 2006; Fridman et al. 2005). The composition and temperature of atmospheric pressure plasmas are produced in a wide range. There are many types of plasma source such as coronas, glows, plasma jets and plasma torches, as shown in Fig. 42 (Woedtke et al. 2013). These plasma sources include power sources are easy handling, relatively inexpensive, compact; therefore, they are suitable for bio-applications with capability of creating chemically active species near room temperature.

Corona discharge is a type of discharge that occurs when a high voltage is applied using electrodes shaped like needles, fine wires, or knife edges and ordinary flat plate-shaped electrodes. Due to the extremely biased electrode shape, the applied electric field is concentrated near the electrode with the smaller surface area. Due to its local high electric field, electrons can easily obtain the high energy required for ionization, so that corona discharge is easily obtained under atmospheric pressure. Generally, the corona discharges can be generated with strength of several to several tens of $\mathrm{kV}$ using DC or AC or pulse power supplies. Schematic diagrams of

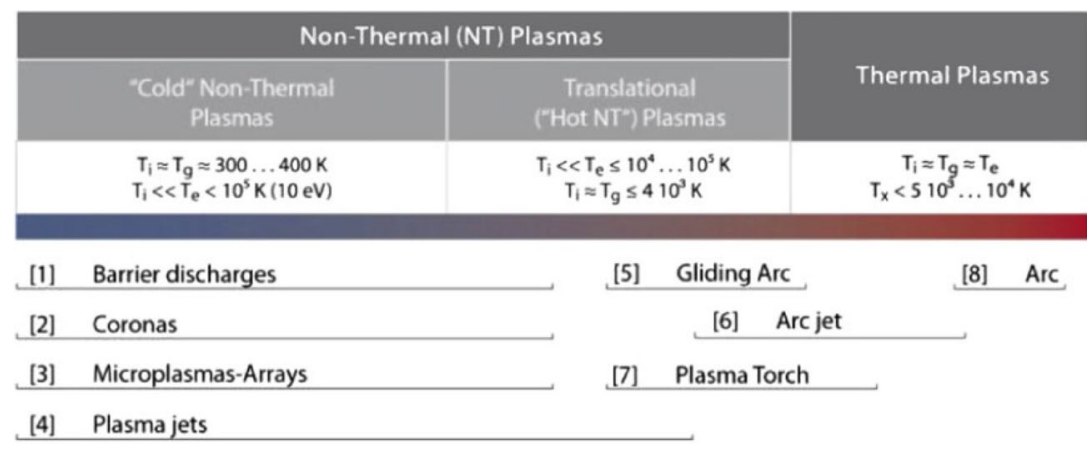
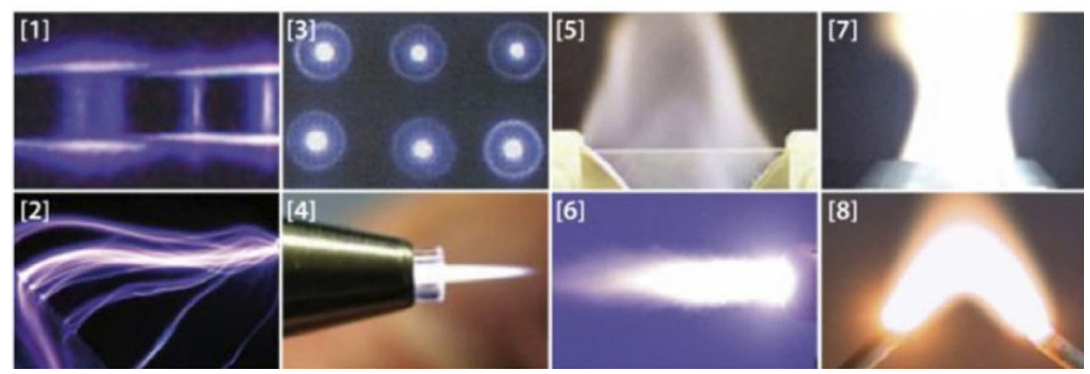

Fig. 42 Photographs of non-thermal and thermal plasmas in atmospheric gas pressure (Woedtke et al. 2013) 


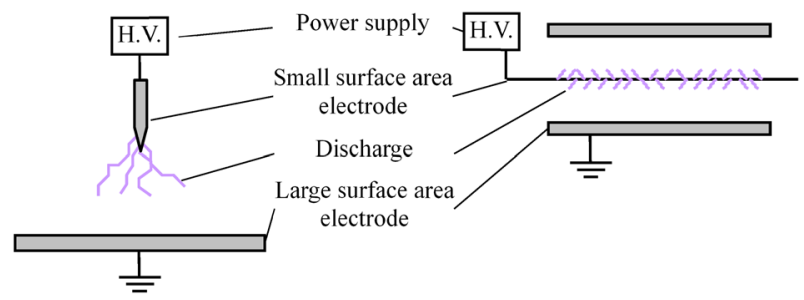

Fig. 43 Schematics of corona discharge apparatus (Tanaka 2021)

the corona discharge apparatus are shown in Fig. 43 (Tanaka 2021). Today, corona discharges are mostly applied in many industrial applications owing to its easily handling (Chang et al. 1991). A corona discharge can be used to generate charged particles such as electrons and ions. and chemically active species such as ROS and RNS. A corona discharge can also be used as an electrostatic precipitator (ESP) (Mizuno 2000). A corona discharge is also used for abating airborne particulates as agricultural application in post-harvest phase as same manner with ESP (Koide et al. 2013).

Dielectric barrier discharge (DBD) occurs when a dielectric such as glass is inserted between the electrodes so as to cover the electrode surface and a high voltage is applied. Schematic diagrams of the DBD apparatus are shown in Fig. 44 (Tanaka 2021; Eliasson and Kogelschatz 1991a). The discharge generated by the left side in Fig. 44 is so-called silent discharge, and thin linear discharges are sparsely generated over the entire electrode in the silent discharge. Since the discharge occurs only sparsely and the discharge gap is generally only a few $\mathrm{mm}$, silent discharge is not used for surface treatment. However, silent discharge is used as the most energy efficient ozone generation method (Kogelschatz 2003). As shown on right side in Fig. 44, the device of the electrode structure made it possible to almost uniformly generate DBD over a relatively wide area. Since the shape of the generated discharge is similar to corona or silent discharges, it is difficult to use this discharge for the completely uniform surface treatment, but it is considered to be more effective than corona discharge as a source of radicals and electrons (Eliasson and Kogelschatz 1991b). Although the DBD's electrodes are more difficult to make than corona discharge apparatus, it is worth considering the use of DBD for agriculture.

Surface discharges are also produced using configurations with thin electrodes on a surface of dielectric layer and a counter electrode on its reverse side. Usually, a knife edge thin plane shapes have been used as metal electrodes to generate corona type surface discharge homogeneously. Recently, mesh electrodes (e.g., multi-patch

Fig. 44 Schematics of dielectric barrier discharge apparatus (Tanaka 2021)

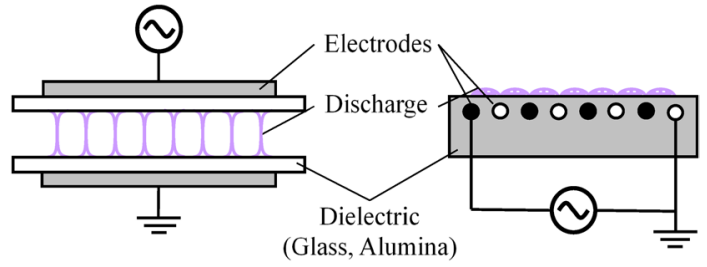


electrodes) are commonly used to produce many microplasmas array in a wide area (Woedtke et al. 2013). The multi-patch electrodes work for confinement of microplasmas in region of several millimeters thickness effectively on the dielectric layer. Coplanar configurations are also used in generation of surface discharge and plasma jet (Gibalov and Pietsch 2000). Many types of surface discharge have been used in many agricultural applications, e.g., promotion of seed germination, inactivation of bacteria on the surface of seeds and agricultural products (Toyokawa et al. 2017; Koga et al. 2016).

Atmospheric plasma (APP) jets are actively used in many bio-applications for medical, agriculture and food safety. Many arrangements of electrode and voltage waveforms are employed for APP-jet; however, the coplanar arranged DBDs are commonly used (Woedtke et al. 2013; Laroussi et al. 2017). In plasma jet, helium or argon gases are frequently used as working gas, because the APP-jets can be operated with low voltage, easily extended several centimeters from the tube tip, etc. AC or pulsed high voltages are generally used in driving the APP-jets, and are applied to one or two metal electrodes winding on the outside surface of dielectric tube. APP-jets can also be generated with corona or glow discharges in vicinity of the high-voltage metal needle tip as electrode located in dielectric tube, as shown in Fig. 45 (Kieft et al. 2004; Stoffels et al. 2006). This type of APP-jet is called a 'plasma needle', which is developed by Stoffels. The working gas is injected into a small diameter dielectric tube, and is ionized and excited at tip of a thin needle electrode applied voltage at $13.56 \mathrm{MHz}$. The plasma can be extended by increasing gas flow rate to several millimeters from the tip. In general, rare gases (He or Ar) are employed as working gas in APP-jet. However, other gases (i.e., oxygen, nitrogen, and water vaper) are sometimes mixed and injected into the rare gases to create various chemically active species such as RNOS (Reuter et al. 2018).

Some agricultural applications require a production of plasmas at gas-liquid boundary or in the liquid such as liquid fertilizer, because the chemical reactions should be induced in the liquid phase (Park et al. 2013; Takahata et al. 2015). The chemically active species and charged particles in plasmas are penetrated from gas into liquid thorough the gas-liquid boundary. Various plasma chemical reactions occur at gas-liquid boundary depending on the plasma parameters (e.g., input energy, electron and gas temperatures), discharge types
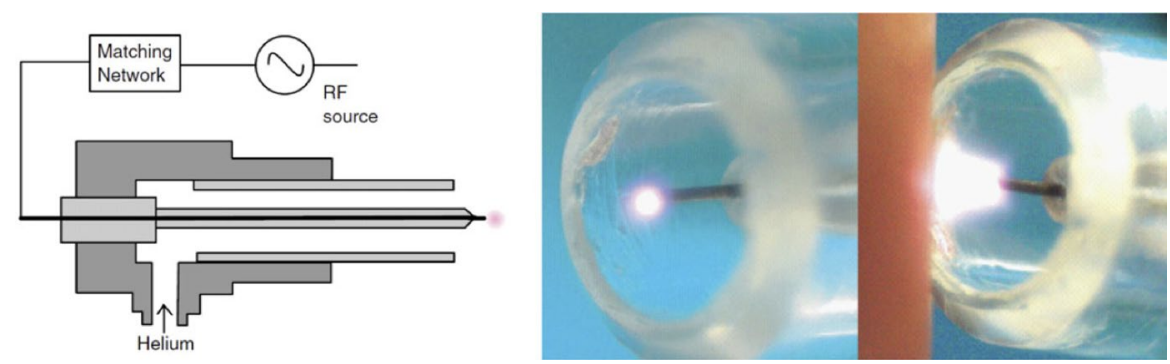

Fig. 45 (Left) Schematic of the plasma needle, its photo in unipolar (middle) and bipolar (right) voltage operations (Kieft et al. 2004; Stoffels et al. 2006) 
(e.g., corona, glow and arc) and gas-liquid compositions. As the results, various charged particles and chemically active species are produced on the boundary of liquid, and then they can penetrate and dissolve into the liquid. The penetrated or dissolved chemically active species and charged particles contribute to chemical and biocidal reactions in the liquid (Lules et al. 2014; Bruggeman and Leys 2009).

Recently, these gas-liquid plasmas have been paid much attention because of their potential for agricultural applications including the direct plants or seeds exposure by non-thermal plasmas. An interaction of non-thermal plasmas to a plant can be expressed as a gas-liquid situation, in which the discharge plasmas are produced in humid air (cultivation environments) or on the wet surface of the plant organs. For liquid surface exposure by plasmas, the surface discharges are actively used on the liquid surface using pulsed voltage. The surface discharges initiate on the vicinity of high-voltage point electrodes (needles) and propagate toward radial directions (Lules et al. 2014). APP-jets are also used to irradiate into the liquid surface for supplying chemically active species. Direct discharges in the liquid are also sometimes employed in the agricultural applications especially in nitrogen fixation in cultivation bed of plants. The direct discharges in the liquid are commonly operated by pulse voltage because of reducing ohmic loss, i.e., conductive loss in the liquid solution. The gas injections or bubbling in the liquid are actively employed to reducing the conductive loss of the liquid solution and enhancing penetration and dissolve of chemically active species into the liquid, as shown in Fig. 46 (Takahashi et al. 2010). While various chemically active species are generated in the plasmas on the boundary of gas-liquid, hydroxyl radical, atomic oxygen, ozone and hydrogen peroxide are commonly accepted as dominant ROS in the processes in many applications such as inactivating bacteria, as an illustration shown in Fig. 47 (Takahashi et al. 2012). Nitrogen-based RNS are also actively used in the applications. The accepted dominant species are nitric oxide (NO) and its derivatives formed nitrites $\left(\mathrm{NO}_{2}{ }^{-}\right)$, nitrates $\left(\mathrm{NO}_{3}{ }^{-}\right)$and peroxynitrites $\left(\mathrm{ONOO}^{-}\right)$for the applications such as plant growth enhancement.

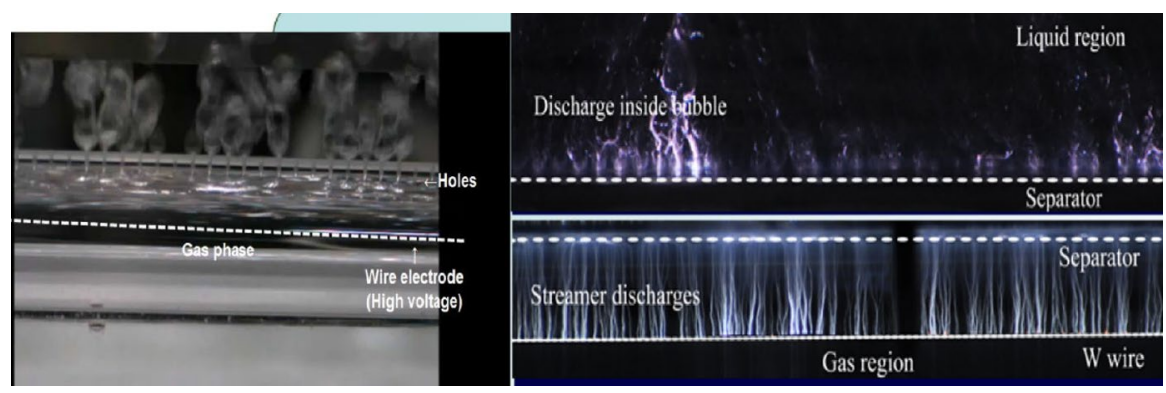

Fig. 46 (left) Photograph of air bubble in liquid injected through small hole array and (right) discharges propagation from wire electrode to air bubble through the holes of separator 


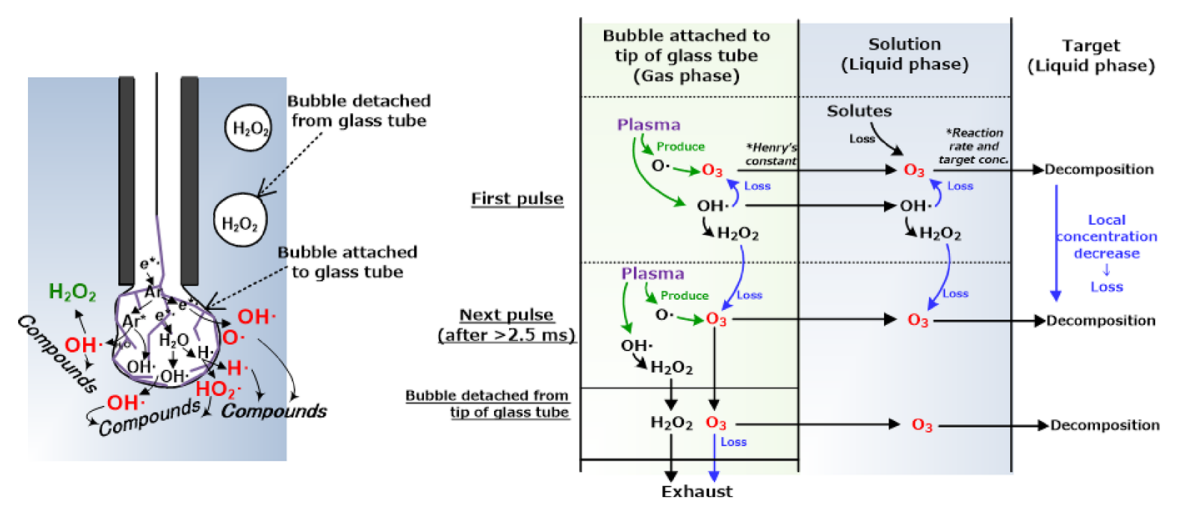

Fig. 47 Illustration of chemical reactions in gas and liquid (water) by plasmas in the bubble injected into the liquid

\section{Plant growth promotion using high voltage and plasma}

Pulsed power technologies including pulsed power and discharge plasmas are tried to use as pre-harvest applications of agriculture such as promotion of seed germination, seedling growth and fruiting body formation (Takaki et al. 2019). In agriculture, the promotion of plant seed germination, growth enhancement of seedling and fruiting body formation of mushroom can contribute to reduce culturing periods and to increase the production yield. Treatments using the pulsed power including high-voltage and time-modulated plasma technologies have some advantages such as without requiring chemical agents, non-thermal process, and that foods enclosed in bags can be inactivated (Cullen et al. 2018). The pathways of promotion of seed germination and plant growth by high voltage applying have been investigated using biochemical methods (Mildaziene et al. 2018). Physical phenomena in biological cells caused by intense PEFs exposing have used in various applications as biotechnology (Akiyama and Heller 2017). These electrical stimuli induce either destroy the biological cells or promote their metabolism and activity. These effects are selected by the intensity such as electric field strength, exposure time (i.e., input energy) of the stimuli (Eing et al. 2009; Song et al. 2020). This section overviews research on plant growth promotion using stimuli by either an electric field or a discharge plasma based on pulsed power technologies.

\subsection{Seed exposure by electric field and plasma}

It had been reported from Greek period that lightning induced a dormancy break, germination promotion of plant seed, and rapid formation of fruiting body of mushroom. A seed is a type of dormant state for plants, in which abscisic acid (ABA) is dominant plant hormone to keep dormancy. The seed germination is defined as a state of dormancy break, in which gibberellic acid (GA) is dominant plant hormone to wake up to grow. About the germination rate of the plant seeds, radish and 
Arabidopsis have almost $90 \%$ of seed germination rate in optimum condition for the germination. However, the germination rates of cider and lacquer tree are very low of lower than $20 \%$. Suppression factors of seed germination are infection of pathogenic bacteria and the plant hormones mainly ABA. The pulsed power technologies can be used for improving germination rate and seedling growth rate through inactivation of the pathogenic bacteria and stimulating to activate metabolism of plant seeds. The electrical stimuli to plant seeds can either destroy (or morphological change) the seeds or promote their germination and seedling growth rate, which depends on strength of the stimuli. This section describes about studies on seed germination using electrical stimuli either by electric field or plasma exposure.

\subsubsection{High-voltage direct stimuli on seed for germination promotion}

DC and AC electric field were exposed to plant seeds as electrical stimuli for promotion of seed germination and seedling growth. Okumura et al. applied to DC voltage of $250 \mathrm{~V}$ to Daikon radish seeds for enhancing germination rate (Okumura et al. 2012). The parallel plane electrodes were used in the experiment with gap distance of $10 \mathrm{~cm}$. It was confirmed that the germination rate increased with DC electric exposure. They also confirmed that the length and mass of the mature radish root also increased by the exposure. Moon et al. confirmed that the water absorbance of the seeds and the levels of antioxidative substances increased by DC electric field exposure (Moon 2000; Zhang and Hashinaga 1997). Kadowaki and Kurisaka evaluated the effect of DC electric field exposure to seeds on Arabidopsis thaliana growth. The germination rate and seedling growth rate increased with the DC electric field exposure. They also confirmed that the of stem length of the mature plant was affected by the exposing DC voltage polarity (Kadowaki and Kurisaka 2013). Huang et al. reported that germination of tomato seeds was induced by exposure to AC electric field of $4-12 \mathrm{kV} / \mathrm{cm}$ in strength and also by exposure to magnetic field of 3-100 $\mathrm{G}$ with an irradiation period from 15 to $60 \mathrm{~s}$. The germination of the seed increased 1.1-2.8 times compared with that of the control (without exposure). However, the seed germination was suppressed at strength of electric field larger than $12 \mathrm{kV}$, and at irradiation periods longer than $60 \mathrm{~s}$ (Huang et al. 2006).

Intense pulsed electric fields (PEFs) were also exposed to plant seeds as electrical stimuli for promotion of seed germination and seedling growth. $\mathrm{Su}$ et al. reported that an effect of nanosecond PEF (nsPEFs) exposure on the early metabolic activity and growth of Haloxylon ammodendron, as shown in Fig. 48 (Su et al. 2015). The strength of pulse electric field was changed to $30 \mathrm{kV} / \mathrm{cm}$ with $100 \mathrm{~ns}$ pulse width. Fifty seeds were immersed to the water in cuvette. The experimental result showed that seedling growth was significantly improved at $20 \mathrm{kV} / \mathrm{cm}$ nsPEF exposure. However, the germination rate decreased by nsPEF exposure at $30 \mathrm{kV} / \mathrm{cm}$. Eing et al. also reported the effects of nsPEFs exposure on germination of Arabidopsis thaliana seed. The electric field strength was changed in range from 5 to $50 \mathrm{kV} / \mathrm{cm}$ with pulse width in range from 10 to $100 \mathrm{~ns}$. The results showed that the optimum input energy density for promotion of seed germination was obtained as $100 \mathrm{~J} / \mathrm{kg}$ (consisted of 

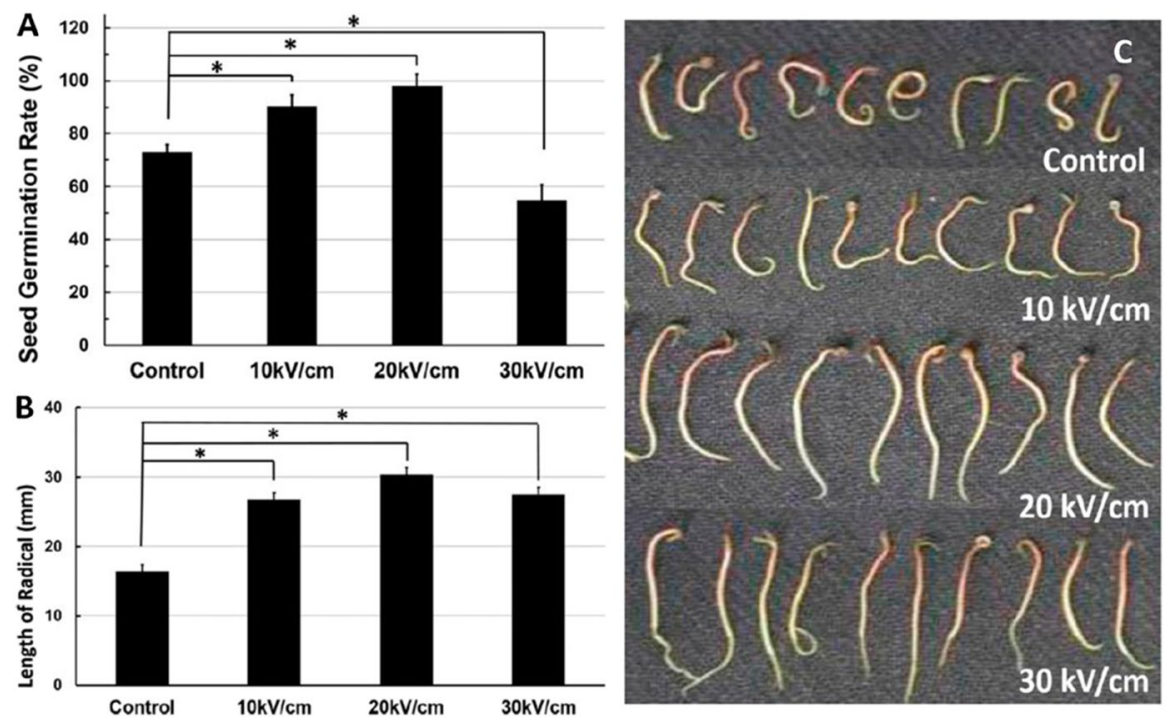

Fig. 48 A Seed germination rate of $H$. ammodendron after $24 \mathrm{~h}$ of incubation for various strength nsPEF exposure. B, C Radical length of $H$. ammodendron seeds after $48 \mathrm{~h}$ of incubation (Su et al. 2015)

$5 \mathrm{kV} / \mathrm{cm}$ in strength, $100 \mathrm{~ns}$ in pulse width and 10 times of exposure) (Eing et al. 2009).

The pathways of promotion of seed germination and seedling growth by electrical stimuli caused by electric exposure have been reported some researchers. Nitric oxide (NO) is accepted as most important signaling molecule inducing various physiological functions in plants, which contributes for seed germination, seedling growth, root organization, leaf development, and alternative oxidase. Especially in the stage of seed germination, lateral root development is induced by low concentration of NO. Su et al. confirmed that NO and the oxidization-reduction potential (ORP) inside the seed cells were positively related to the early growth effects of $H$. ammodendron seed by electric field exposure. The free calcium $\mathrm{Ca}^{2+}$ bursts in plant cells were induced by nsPEF exposure. The free calcium $\mathrm{Ca}^{2+}$ is a strongly related to the growth and development in plants. Therefore, the electrical stimuli by nsPEF exposure induces free calcium bursts into intercellular cytoplasm of plant seed cells, nitric oxide synthase, following those process, activation and production of much more endogenous NO, which finally trigger the cell signaling pathway and promotes the seed germination and pre-growth of plants, as shown in Fig. 49 (Su et al. 2015).

Dymek et al. investigated that the metabolic responses in germination of barley seeds upon by PEFs exposure. Malting barley seeds were immersed in an aerated water for $24 \mathrm{~h}$, following the pre-treating process, the seeds were treated by PEFs exposure with various strengths of voltage. The barley seed exposure by PEFs affects radical production in the plants without significant change of metabolic activity (Dymek et al. 2012). Wang et al. reported that the seeds vitality indices (membrane lipid peroxidation, protective enzymes of SOD and peroxidase) increased significantly by applying a PEFs to aged rice seeds (Wang et al. 2009). 


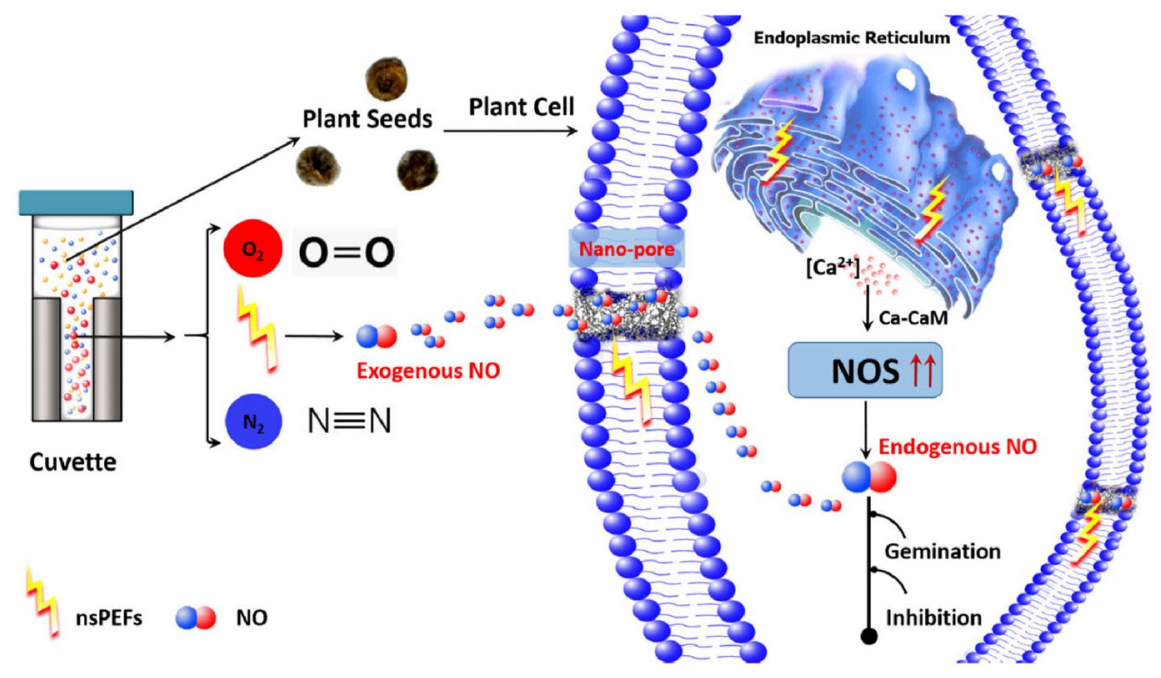

Fig. 49 Illustration of the signalling network involving Nitric Oxide (NO) pathway triggered by nsPEFs applying to H. ammodendron seed. (Su et al. 2015)

\subsubsection{Seed exposure by plasma for germination promotion}

Non-thermal plasmas (cold plasmas) are produced in many arrangements such as DBD, APP-jet, surface discharges, corona and glow discharges as described in Sect. 4.4 .4 (Weltmann et al. 2010). The non-thermal plasmas involve a complex mixture of electrons, positive and negative ions, neutral atoms, radicals, and chemically active species such as reactive oxygen and nitrogen species (ROS, RNS), which has both positive and negative effects on biological systems, depending on the input energy and power source of treatment (Attri et al. 2015). The plasma treatment directly or indirectly (called as plasma-activated medium; PAM, plasma-treated water; PTW, etc.) can activate or inactivate the plant metabolism, through biochemical reactions and biological signaling in the cell following cell membrane oxidization stress, protein oxidation, etc. (Graves 2012; Wilson et al. 2008; Torres 2010).

Dubinov et al. investigated that the seed germination and seedling growth of grain crops for various input powers of atmospheric plasma exposure. The effect of irradiation to grain crop seeds by air glow plasma as electrical stimulus was confirmed as germination and growth enhancement (Dubinov et al. 2000). Tong et al. confirmed the promotion of germination and early growth of andrographis paniculate seeds by air plasma irradiation (Tong et al. 2014). Dobrin et al. also reported that the enhancement of germination and early growth of wheat seeds by plasma exposure using air (Dobrin et al. 2015). The similar stimulation effects on seed germination for several species such as peanut (Li et al. 2016) and soybean ( $\mathrm{Li}$ et al. 2014) were confirmed using plasma exposure. $\mathrm{Li}$ et al. showed that the germination and vigor indices significantly increased by 14.7 and $63.3 \%$, respectively, by the plasma irradiation. About effect on growth rate of plants, the increasing rates of shoot length, shoot dry weight, root length and root dry weight were 13.8, 22.0, 21.4 and 27.5\%, 
respectively, compared with the controls, in which without plasma irradiation ( $\mathrm{Li}$ et al. 2014). There are review reports which shows the effect of non-thermal plasma exposure on plant seeds for various working gases, various plasma sources (Attri et al. 2021).

Pathways for promotion of germination and growth enhancement of plants by plasma exposure have been investigated by some researchers. Alves et al. investigated that a relationship between germination and water uptake of Erythrina velutina seeds using APP-jet exposure. They indicated that the pathway of water uptake was determined with hilumt and micropyle, and the water uptake was improved by the plasma treatment (Alves et al. 2016). Henselová et al. reported that the variation in enzyme activities of catalase (CAT), guaiacol-peroxidase (G-POX), superoxide dismutase (SOD) and dehydrogenase (DHO) of the root of maize and the root microscopic structure after plasma exposure to maize seeds using diffuse coplanar surface barrier discharge (Henselová et al. 2012). Li et al. reported the results of experiment in which soybean seeds were irradiated by atmospheric helium plasma. The germination rate and speed were enhanced due to increasing water absorption of the seeds, utilizing seed reserved substances and changing glucose and protein amounts in the seeds ( $\mathrm{Li}$ et al. 2014). Zhang et al. evaluated the promotion of lentil germination and stem growth using plasma-activated water (PAW). Hydrogen peroxide and nitrate in the plasma-activated water mainly contributed to germinate (breaking seeds from dormancy) through the NO radicals production process in the seeds (Zhang et al. 2017).

Plant hormones (phytohormone) played a key role in seed germination and seedling growth. Plasma irradiation to seeds also affects the concentration of plant hormones related to the germination and seedling growth. Stolárik et al. showed that the changes in endogenous hormones like auxins, cytokinins, and their catabolites by plasma irradiation related to the growth enhancement (Stolárik et al. 2015). Reactive species ROS works as oxidative stress for seed germination and dormancy in plants (Attri et al. 2021). Choudhary et al. confirmed that the increase of metabolism in seeds is mainly caused by ROS production in hydrated seeds, however, lipid peroxidation work as a source of ROS in dry seeds. Notes that ROS have an important role in association with endosperm deterioration, pathogen defense, seed reserve mobilization, and programmed cell death during seed germination (Choudhary et al. 2020). In general, ROS interacts with plant hormones such as gibberellic acid (GA), abscisic acid (ABA), and ethylene which are strongly associated to seed dormancy and germination, as shown in Fig. 50. ABA activates the ROS scavenging enzymes to inhibit the germination. In the contrary, GA inhibits in activity of ROS scavenging enzymes, then germination is promoted. Therefore, the GA and ABA ratio can be used as index of seed germination and dormancy. The high GA/ABA means promoting a germination, on the other hand, low GA/ABA means keeping dormancy state. Zukiene et al. confirmed that plasma irradiation to sunflower seeds induced a change in GA/ABA, as shown in Fig. 51 (Zukiene et al. 2019). Degutytè-Fomins et al. also reported that the increase of GA concentration and decrease of ABA concentration in radish sprouts by plasma irradiation using DBD plasma source (DegutytèFomins et al. 2020). 


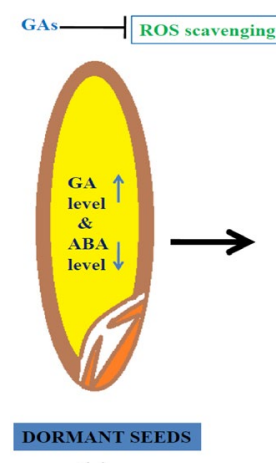

(a)

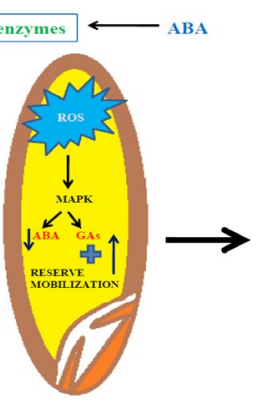

(b)

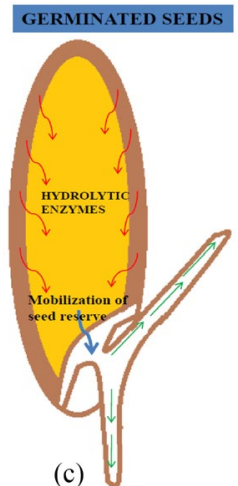

(c)

Fig. 50 Illustration of model for role of ROS in seed dormancy and germination. (Choudhary et al. 2020)
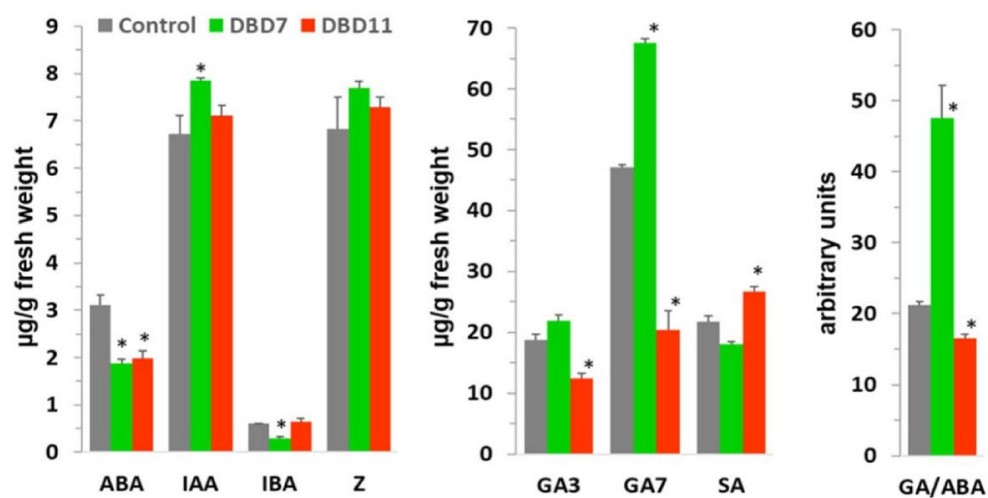

Fig. 51 Amount of plant hormone in dry seeds of sunflower. ABA, GA3, GA7, IAA, Z, and SA indicate abscisic acid, gibberellin 3, gibberellin 7, indole-3-acetic acid, zeatin, and salicylic acid, respectively. GA is GA3 and GA7. (Zukiene et al. 2019)

\subsubsection{Seed exposure by plasma for seedling growth}

In general, seeds and sprouts of crop plants have been treated using chemical agents such as fertilizers and phytohormones for promotion of the seedling growth. The promotion of growth contributes reducing cultivation cost by reducing period to harvesting and providing a stable supply of agricultural products to markets by increasing yielding rate of crop. For example, gibberellic acid (GA) is a one of phytohormone which works promotion of seed germination, growth and fruition, and is frequently used in crop culture as chemical agent for promotion of seedling growth. Non-thermal plasma irradiations are also tried to be used for promotion of plant growth in agriculture fields.

Some researchers reported the promotion of seedling growth either by plasma irradiation to plant seeds (Ji et al. 2016; Sivachandiran and Khacef 2017; Koga et al. 
2016), or seed exposure by pulse electric fields (Su et al. 2015; Eing et al. 2009). About the plasma irradiation to the plant seeds, non-thermal atmospheric plasmas (DBD, APP-jet, corona and surface discharges) are mainly used with air-feed and $\mathrm{AC}$ or pulse voltage drive. However, sometime low-pressure plasmas (ICP, CCP) are used as stimuli with various gases used in plasma generation to clarify the dominate radicals for the promotion. The growth promotion effect of plasma irradiation was confirmed by size of plant parts (stem, root, leaf and scape). These plant sizes increased in comparison with control (without plasma irradiation) by plasma irradiation to the seeds (Ji et al. 2016; Sivachandiran and Khacef 2017; Kitazaki et al. 2014). In addition, the growth promotion effect of plasma irradiation was also evaluated with crop yield. Some researchers confirmed that the crop yields (including size of harvested products) increased by plasma irradiation to the seeds (Koga et al. 2016; Zhou et al. 2011). The pathways (mechanisms) of the growth promotion were also investigated by some researchers with analysis of growth factor and gene expression analysis. Hayashi et al. reported that the dominant active species of plasmas for the growth promotion were reactive nitrogen species (RNS) using low-pressure ICP with various feed gases (Hayashi et al. 2015). They also reported that effects for the growth promotion depended on dose of the active species flux to exposure (Hayashi et al. 2015). Yin et al. described that the one of pathway for growth promotion included the removal of the abscisic acid (ABA) from seed surface by plasma irradiation (etching), because the increase of germination rate related to seedling growth (Yin et al. 2005).

Many kinds of plant have been used as specimens for evaluation of growth promotion by plasma exposure to the plant seeds. Arabidopsis thaliana have been frequently employed as a model plant because its genes was completely analyzed and those functions were also clarified. In addition, Raphanus sativus L. (radish sprout) were also frequently used as specimens because of easily handling owing to the larger size of its seeds compared to those of other plants. Sarinont et al. investigated the effect of plasma irradiation using various kind gases (air, $\mathrm{N}_{2} \mathrm{O}_{2}$, and $\mathrm{NO}+\mathrm{N}_{2}$ gas mixture, He, Ar gases) on growth of Raphanus sativus L. They indicated that active oxygen species (ROS) including plasmas of air, $\mathrm{O}_{2}$, and $\mathrm{NO}+\mathrm{N}_{2}$ gases worked for promotion of plant growth. In contrary, the plasmas of $\mathrm{N}_{2}, \mathrm{He}, \mathrm{Ar}$ did not contribute to enhance the plant growth (Sarinont et al. 2016). Sivachandiran et al. confirmed the increase of crop yield (including weight and size of agricultural products) as growth enhancement by plasma irradiation to the seed, as shown in Fig. 52 (Sivachandiran and Khacef 2017). Similar effects of plasma irradiation to the plant seeds on growth enhancement were confirmed by some research groups (Sera et al. 2017; Park et al. 2016). Zhou et al. evaluated the effects using tomato seeds exposure by atmospheric pressure plasma driven with $4.7-6.8 \mathrm{kV}$ voltage. The plasma irradiation to the tomato seeds resulted the promotion of germination, and increasing length and diameter of cropped tomato fruit (Zhou et al. 2011). Yin et al. used the magnetized plasma as electrical stimuli of tomato seeds for growth enhancement. The seed exposure by magnetized plasma effectively works for increasing harvest yield of tomato fruit with $20.7 \%$ (Yin et al. 2005). 


\subsubsection{Sterilization of seed surface}

Sterilization of seed surface is important issue for crop cultivation because of reducing infection risk of bed soil and plants itself. In common, chemical agents such as fungicides have been employed to killing pathogenic bacteria on seeds. However, the alternative treatments for the sterilization instead of chemical agents are required for environmental protection. Sometimes hot water has also been used to disinfect the bacteria on seeds. However, the hot water treatments have to be carried out just prior to seedling, because the hot water treatments induce seed germination. Therefore, some researchers have been investigated about high voltage and plasma sterilizations of seed surface to promote seed germination and seedling growth.

Morar et al. investigated the sterilization on Phaseolus vulgare beans which was contaminated with Colletotrichum lindemuthianum by exposure with electric fields in range from 2 to $16 \mathrm{kV} / \mathrm{cm}$. The electric field was produced by $\mathrm{AC}$ voltage with a frequency of $50 \mathrm{~Hz}$ and the electric field irradiation time was set in range from 1 to $30 \mathrm{~s}$. The experimental results showed that more than $99 \%$ of the seeds were germinated with treatment of electric field irradiation, although only $30 \%$ of nontreated seeds were germinated. The yielding rate as cropped weight from bean plants increased by the electric field treatment of beans. They also carried out the field

Fig. 52 Long period effects of plasma irradiation to seeds on plant growth. Photos of a tomato and $\mathbf{b}$ pepper plants after on 60 days cultivation. Reproduced from (Sivachandiran and Khacef 2017)
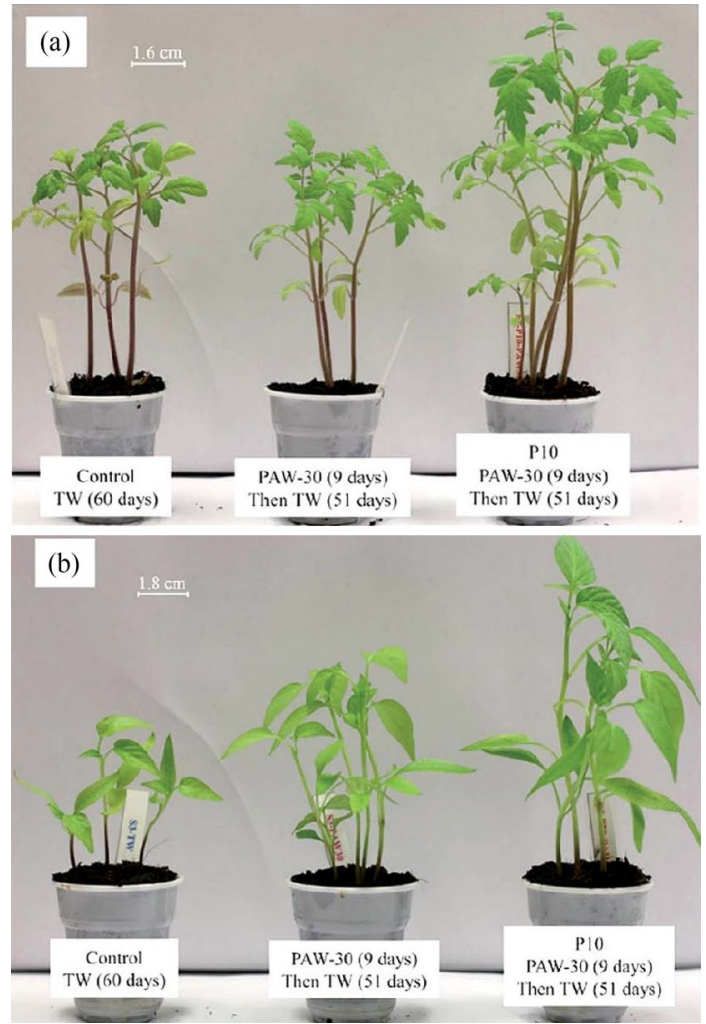
tests. The results showed that all plants were prevented from the plant disease by bacteria infection by electric field treatment of seeds. The dominant factor of sterilization was estimated as ozone which was produced by void discharges occurred in void between seeds (Morar et al. 1999).

Randeniya and Groot employed a reactive plasma for seed disinfection. They confirmed that the pathogenic bacteria on the surface of radish, rice and poppy seeds were inactivated by reactive plasma irradiation through oxidization of bacteria by charged and neutral particles containing the plasmas (Randeniya and Groot 2015). For sterilization on seeds for disinfection, atmospheric plasmas are commonly used such as DBD and corona discharge plasmas. Butscher et al. reported that the inactivation of bacteria depends on configuration of the electrodes and humidity of the feed gases (Butscher et al. 2016). Khamsen et al. employed micro-corona discharge for sterilization. They confirmed that bacteria on rice (Oryza sativa L.) was inactivated with irradiation by the micro-corona discharge with high material compatibility (Khamsen et al. 2016).

Low-gas pressure plasmas were also sometimes used for sterilization of the seeds. Radio-frequency (RF) inductive coupled plasmas (ICP) and microwave discharge plasmas (MWP) were used as low-pressure plasmas for inactivation of seed-borne bacteria and mold on seed surfaces (Sera et al. 2013; Ono et al. 2017). Ono et al. carried out that cabbage seeds contaminated by Xanthomonas campestris pv. Campestris $(X c c)$ were irradiated by low-pressure oxygen and air plasmas with the vibrating seed stirring device to penetrate the active species into all seeds. The results showed that the inactivation efficiency of the plasma irradiation with the stirring device was more than ten times higher than that without the stirring device (Ono et al. 2017).

In general, seed germination rate significantly decreases by infection of seeds with mold and bacteria. For example, radish sprout seeds germination rate decreases below $50 \%$ by infection of mold and bacteria. Mitra et al. confirmed that the germination and growth rates of plants increased by seed disinfection treatments using atmospheric and low-pressure plasmas, as shown in Fig. 53. Surface-borne microorganisms on seeds were inactivated by irradiation with cold atmospheric plasma (CAP) using ambient air. The inactivation efficiencies were $1 \log$ and $2 \operatorname{logs}$ by plasma irradiation of 2 and $5 \mathrm{~min}$, respectively. The germination and growth rates increased to $89.2 \%$ and 7.1 seeds/day, respectively, by the plasma irradiation. In addition, the average period required for germination decreased to 2.7 days (Mitra et al. 2014).

Similar results were obtained and reported by some researchers. Matra reported that the length and dry weight of seed plant roots increased by inactivation of pathogenic bacteria on radish sprout seeds by atmospheric pressure plasma. The increase rate of root length and weight were affected by the input power and period of plasma treatment (Matra 2016). Sera et al. employed that low-pressure microwave plasma for irradiation to poppy seeds for sterilization of pathogenic bacteria. The MWP irradiation period of was set to be less than 10 min with a input power of $500 \mathrm{~W}$ using an oxygen and argon gas mixture. The results showed that the germination rate increased to $115 \%$ after 4 days cultivation by MWP irradiation (Sera et al. 2013). Jiang et al. monitored many indexes such as seed germination, growth rate, nutrient 


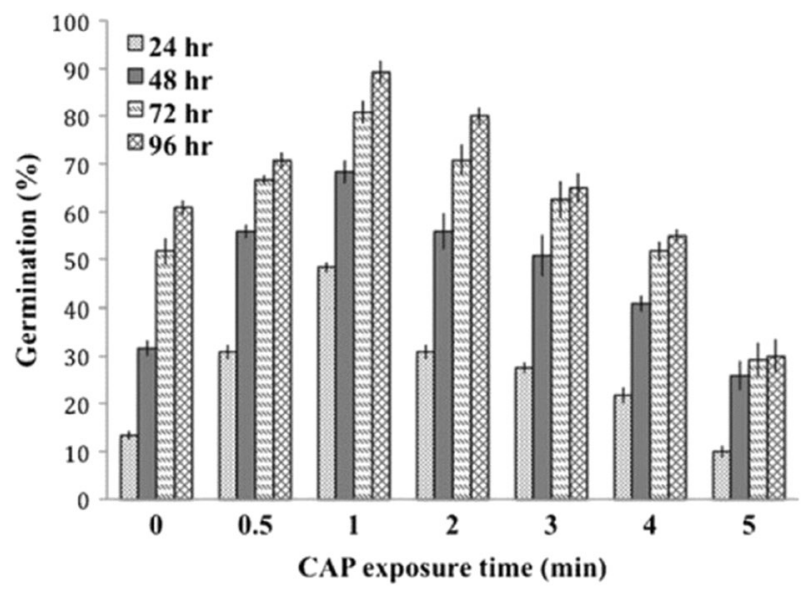

Fig. 53 Influence of cold atmospheric plasma (CAP) irradiation to the seed on germination of Cicer arietinum seeds for various exposure period $(n=6 ; p<0.05)$ (Mitra et al. 2017)

uptake capacity, disease severity, hydrogen peroxide concentration and activities of enzymes (i.e., peroxidase, polyphenol oxidase and phenylalanine ammonia lyase) as an effects of plasma irradiation to seeds of tomato. The results showed that tomato resistance to Ralstonia solanacearum increased with an efficacy of $25.0 \%$ by plasma irradiation to the seeds. Following that, both seed germination and plant growth increased significantly by the plasma treatment of seeds, and plasma-treated seed plants absorbed more calcium and boron than the plants without plasma treatment (Jiang et al. 2014a). Other similar results are reviewed by Sera and Sery (2018).

\subsection{Plant tissue exposure by electric field for vegetative growth promotion}

Phenomena in biological cells exposure by intense electric field have a variety of applications in biotechnology. Proper control of applied electrical energy can cause genomic modifications, stimulate enzyme activity, enhance photosynthesis, control $\mathrm{pH}$ and bacteria levels in the cultivation environment, and increase nutrient uptake, resulting in increased harvest yield. There are two approaches for plant growth enhancement. One approach is based on directly stimulating plant tissues using intense electric field. The other is indirect stimulation in which intense electric fields (including plasmas) are exposed into the bed medium used in plant cultivation. In this session, the direct stimulation of plants using PEF for growth promotion are outlined. After that, the indirect stimulation are described in session 5.3.

\subsubsection{Electric field and ion stimuli for plant metabolism}

Regarding research trends prior to 2000, electrical stimulation of the plant body was mostly done with weak DC current or air ions that were attempts at simulating possible conditions in the natural environment observed in geoelectric field studies as 
mentioned in chapter 2 (Shigemitsu 1999; Barman and Bhattacharya 2016; Wang 2021).

In the early 1960s, Krueger et al. reported that air ions from an electrostatic precipitator can induce growth in higher plants. Ions of either positive or negative charge generated in air induced statistically significant increases in stem length, integral elongation, and wet and dry weight. A possible mechanism is that air ions enhance the incorporation of Fe into cytochrome $c$ (Krueger et al. 1963). Cytochrome $c$ is a small hemeprotein that plays a major role in cell apoptosis. Kotaka et al. found that exposure to air ions results in increased swelling and shrinking of isolated chloroplasts in response to light, which may involve the expenditure of energy from ATP hydrolysis. When chloroplasts were incubated in light, dark, and reilluminated environments, the augmented ATP metabolism increased the swelling rate during the period of pre-incubation and similarly accelerated the shrinking rate during dark incubation. Eventually there is not enough ATP left to support increased swelling of ion-treated chloroplasts upon reillumination. Air ions affect the ATP energy-yielding reaction that proceeds within the chloroplast and is associated with swelling and shrinking (Kotaka et al. 1968).

In 1971, Black et al. found small currents could stimulate or inhibit active ion pumps. The small current also could affect the internal distribution of growth-regulating compounds in tomato plants. Enhancement of plant growth (5-30\%) and K, $\mathrm{Ca}$, and $\mathrm{P}$ uptake were obtained by applying direct currents of 3-15 $\mu \mathrm{A}$ to plant with negative potential of plant to the ground. Reductions in growth occurred at currents of $15 \mu \mathrm{A}$ with positive potential of plant to the ground, or with any currents greater than $30 \mu \mathrm{A}$. It seems likely that the applied electrical current may not only affect ion accumulation but also internal auxin or gibberellin activity. The conclusion is that stimulation of ion uptake and growth via electric current is mainly due neither to increased passive cation uptake nor electroosmosis, nor is it closely related to the reduction potential of each cation. The researchers posit that the changes in growth and ion uptake are likely due to active ion pump stimulation at the root surface or to a redistribution under the electric field of plant growth-regulating substances inside the living tissue (Black et al. 1971).

In the years since, more studies have been carried out using higher power electric fields or shorter application durations. Ye et al. reported that application of PEF with $10 \mathrm{~V} / \mathrm{m}$ in strength at $50 \mathrm{~Hz}$ in frequency induces defense responses and stimulate secondary metabolite accumulation in Taxus chinensis plant cells in suspension culture. They also confirmed that an intracellular accumulation of taxuyunnanine $C$ $\left(T_{\mathrm{c}}\right)$, which is a bioactive secondary metabolite, was significantly increase by $30 \mathrm{~min}$ PEF irradiation to the cells in the early exponential growth phase. PEF treatment can be optimized by strength and exposure period to enhance the desired secondary metabolite production without harmful effects in viability and biosynthetic capacity. Moreover, PEF can change the dielectric properties of cell membrane and can induce the release of intracellular metabolites (Ye et al. 2004). Zvitov et al. observed stomatal opening by applying of low DC electrical field to leaves of Cucumis sativus L. and Commelina communis L. The mechanism for stomatal opening by DC electric field exposure was proposed that the electric field exposure affected turgor pressure of guard cell, as the result, differential of between turgor pressure of guard 
cell and of the surrounding epidermal cells was changed. The applied voltage damages the epidermal cells, causing a decrease in turgor pressure and a loss of pressure against the swelling of the guard cells, leading to the opening of many stomata. In the study, small voltages of $\sim 5 \mathrm{~V}$ were enough to induce opening of stomata in the electric field exposure. However, the higher voltages of $\sim 20 \mathrm{~V}$ led to guard cell death, in addition to stomatal opening. It was confirmed by neutral red staining that the small voltages damaged the epidermal and subsidiary cells and kept the guard cells viable, although the high voltages caused the death of the guard cells as well (Zvitov et al. 2003).

\subsubsection{Pulsed electric field stimuli for plant vegetative growth}

Effects of PEF on photosynthesis in Auxenochlorella protothecoides microalgae was explored by Straessner et al. (2013). High voltage was applied with pulse durations of 100 and $1000 \mathrm{~ns}$ for PEF treatment of microalgae suspensions. The input energy for the treatment was varied between 2 and $78 \mathrm{~kJ} / \mathrm{kg}$ with constant PEF strength of $40 \mathrm{kV} / \mathrm{cm}$. Auxenochlorella protothecoides was much more sensitive to long PEFs, rather to short PEFs, in particular under low input energy condition. A significant influence of PEFs worked on photosystem II (PS II). Microalgae exposed to $1000 \mathrm{~ns}$ pulses started with significantly lower $F_{\mathrm{v}} / F_{\mathrm{m}}$ values, which indicates the maximum photochemical quantum yield of PS II chemistry after dark adaption, and exhibited a notably faster decline over time, in comparison with the experiments with $100 \mathrm{~ns}$. Sonoda et al. also reported an effect of PEFs on photosynthesis in Lactuca sativa L. leaf lettuce (Sonoda et al. 2017). They used low $(0.2 \mathrm{kV} / \mathrm{cm})$ and high $(1.0 \mathrm{kV} /$ $\mathrm{cm}$ ) strengths of PFE at pulse durations of 400 and $500 \mathrm{~ns}$. The effects of PEF treatment were evaluated by photosynthetic electron transport rate (ETR), photochemical quenching (qP; related to the redox state of electron acceptors in photosystem II), and non-photochemical quenching (NPQ; related to heat dissipation of light energy). The experimental results reveled that ETR and $\mathrm{qP}$ of dark-acclimated nursery leaf lettuce increased by applying $0.2 \mathrm{kV} / \mathrm{cm} \mathrm{PEF}$, although the treatment with $1.0 \mathrm{kV} / \mathrm{cm}$ PEF did not affect, as shown in Fig. 54. Conversely, PEF treatment with $1.0 \mathrm{kV} / \mathrm{cm}$ showed in a decrease in NPQ, while the treatment of $0.2 \mathrm{kV} / \mathrm{cm}$ showed

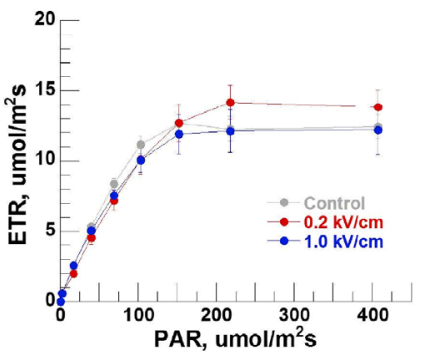

(a) ETR of light-acclimated samples

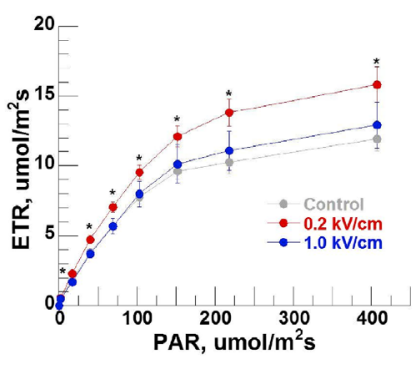

(b) ETR of dark-acclimated samples

Fig. 54 The results of ETR in both conditions (light-acclimated and dark-acclimated) after PEF treatment (Sonoda et al. 2017) 
no significant affect. Low PEFs are considered as improving electron transfer flux through PSII in photosynthesis, while high PEFs inhibit heat dissipation which works as a stress defense, as shown in Fig. 55 (Sonoda et al. 2017).

\subsubsection{Rhizome exposure by pulsed electric field for plant growth}

A rhizome is the main stem of the plant that runs underground horizontally. Plant root cells produce electric fields through ion transporter activity. This ion movement creates a flow of current through the tissue, generating electrical potentials across the membranes of the root cells. During the growth response of the root to gravity, the electrical patterns change. The application of exogenous electrical fields to the root can be used to investigate the relationship between electrical patterns and root growth.

Sonoda et al. investigated the effect of plant root exposure by various strengths (from 0.2 to $2.0 \mathrm{kV} / \mathrm{cm}$ ) of PEFs with $400 \mathrm{~ns}$ of pulse width on growth rate of leaf lettuce (Sonoda et al. 2014). The PEFs were generated between a parallel plane electrodes, which were immersed in the liquid fertilizer. The roots of leaf lettuce were located between the plane electrodes, in which a uniform PEF was applied to the roots. The experimental results showed that leaf lettuce size increased by applying PEFs to the roots with strength from 0.2 to $1.0 \mathrm{kV} / \mathrm{cm}$ after 20 days cultivation from day of the stimulation, as shown in Figs. 56 and 57 (Sonoda et al. 2014). In contrary, the growth

Fig. 55 Effects of PEF irradiation to leaf lettuce evaluated on chlorophyll fluorescence analysis (Sonoda et al. 2017)
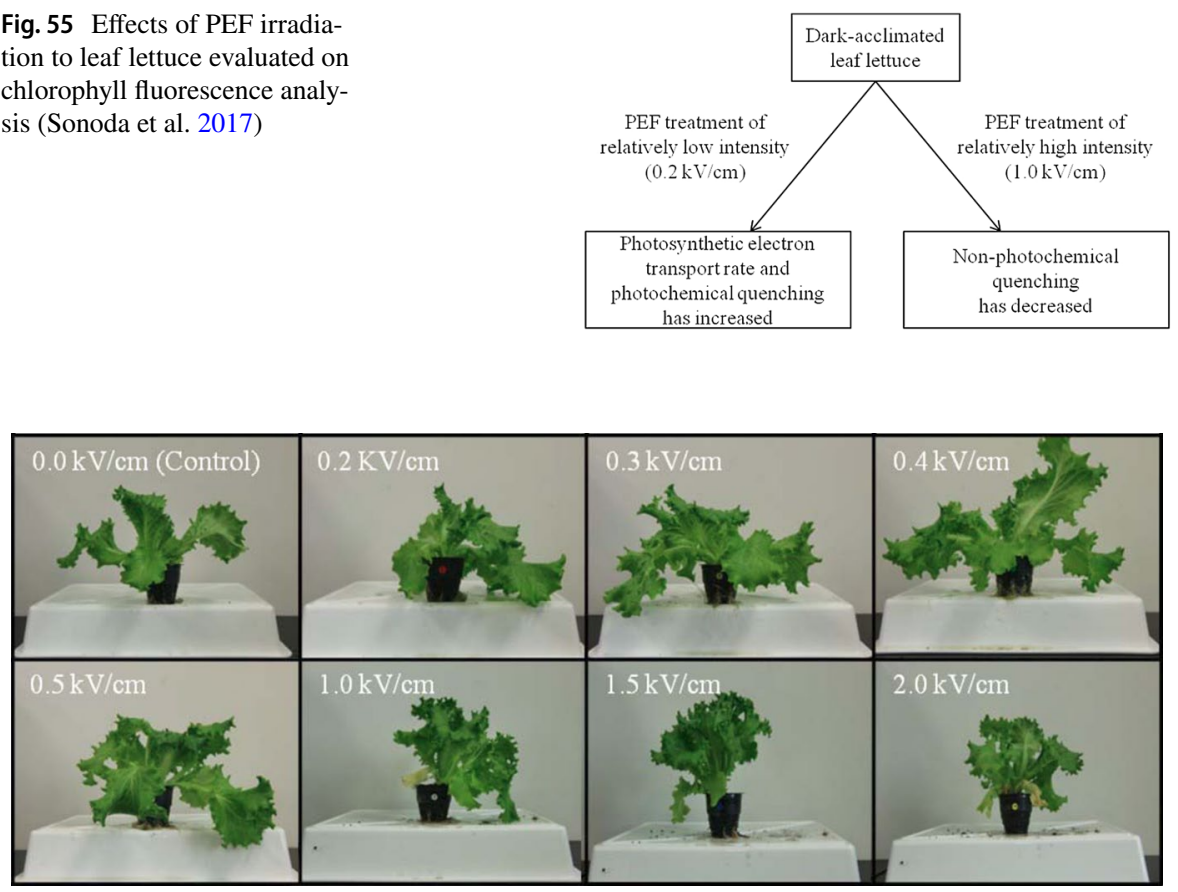

Fig. 56 Typical photographs of leaf lettuce at each electric field strength exposure after 20 days of cultivation. (c) 2014 IEEE. Reprinted, with permission, from (Sonoda et al. 2014). 
Fig. 57 Total fresh weight of leaves per pot for each electric strength after 20 days cultivation. (C) 2014 IEEE. Reprinted, with permission, from (Sonoda et al. 2014).

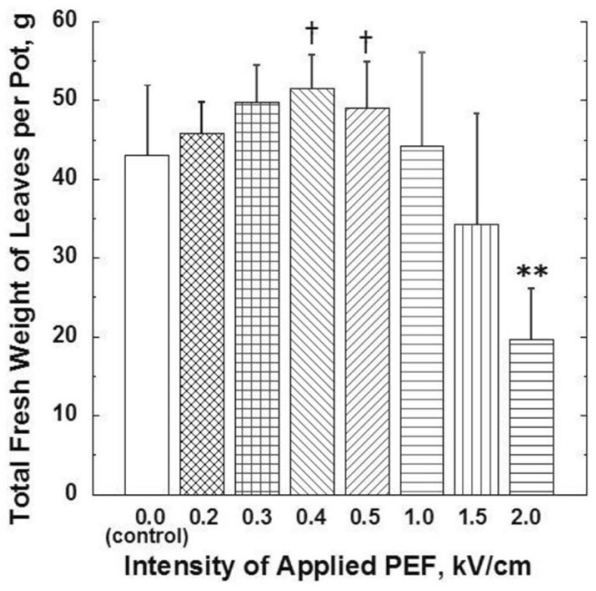

of the plants was suppressed by applying PEF with strength more than $1.0 \mathrm{kV} / \mathrm{cm}$. In addition, roots of the leaf lettuce which induced larger leaf weight grew more robustly than those of smaller leaf weight plants. The PEF applying was also confirmed by chemical analysis that there was no significant influence on chemical properties of liquid fertilizer. These results indicate that PEF does not cause the change in composition of liquid fertilizer, while directly works as stimuli for growth of leaf lettuce.

Another study on effects of PEFs irradiation to plant roots on growth lettuce and hot peppers cultivated in soil was carried out by $\mathrm{Yi}$ et al. The pulse voltage from 2 to $10 \mathrm{~V}$ was applied to electrode immersed in a culture bed soil. They confirmed that the growth of lettuce increased by applying voltage to the soil from 4 to $10 \mathrm{~V}$. The period for growth of hot pepper plant was prolonged compared with that of control plants (without electrical stimulation). In addition, rooting from the cut branch was promoted by the electric pulse irradiation when the fresh branches of hot pepper plants were cut and implanted into the electric field treated soil (Yi et al. 2012). Statsyuk et al. investigated the effect of a pre-planting treatment of seed tubers by PEF on the growth of potato plants. The seed potato was exposed by $20 \mathrm{kV} / \mathrm{m}$ strength PEF with $16 \mathrm{kHz}$ of AC frequency. The PEF treatments was carried out at 3-5 days prior to planting with repetition rate of the modulating pulse pattern of $200 \mathrm{~Hz}$. The pre-planting treatment of seed tubers by PEF did not significantly affect plant height, number of leaves, and fresh weight of above-ground parts of potato plants. However, number of stems per plant increased by the PEF treatment. Number and weight of tubers per plant also increased with averaged increase rate of $27.0,28.3$, and $31.1 \%$ for stem number, number and weight of tubers per plant, respectively, compared to the control plants (Statsyuk et al. 2016).

For clarifying the mechanism of promotion of plant growth by PEF direct stimulation, several researchers reported the results of analysis through experiments. Bovelli et al. carried out the experiment using pulse electromagnetic fields exposure to Nicotiana tabacum $\mathrm{L}$. The results reveled that a primary action was induced at plasma 
membrane level as a signal transduction process through the change of electrical potential. This process also induced an activation of enzymes (Bovelli and Bennici 2000). Other pathway was proposed as perturbations in the metabolism in the cells including the genomic molecules (i.e., DNA or RNA) and proteins. The external electromagnetic fields exposure could also affect ions as Lorenz force and change the flow of transcellular currents. In addition, the electromagnetic fields have potential to reduce calcium ions from cell membranes, resulting that, inducing enzyme activity through the calcium cascade process (Bovelli and Bennici 2000). Ye et al. (2004) suggested that PEF stimulated for activation of a defense response in plant cells, which induced the change of cell membrane dielectric properties. Cai et al. reported that the combination of PEF stimulation and ethephon treatment improved the activity of secondary metabolisms. The concentrations of anthocyanins and phenolic acids in cell cultures increased by $\mathrm{PEF}$ and ethephon stimulations in comparison with those of controls. Moreover, the production of extracellular phenolic acids and 3-O-glucosylresveratrol also increased by the PEF treatment (Cai et al. 2011).

\subsection{Indirect stimulation through improvement of growth environment}

Improving growth rate of crop plants in the planting, seedling and cultivation phases is a key factor for improving harvest yield. Soil have been commonly used in farming for a long time, however, recently hydroponic cultivations have also been expanded for farming many kinds of vegetables and fruits. In hydroponic cultivations, artificial nutrient solutions are used as fertilizer, which are circulated in the cultivation bed for growth enhancement of crop plants. In general, the artificial nutrient solutions for cultivation contain electrolytes such as $\mathrm{K}^{+}, \mathrm{NH}_{4}{ }^{+}, \mathrm{Ca}_{2}{ }^{+}, \mathrm{M}_{2}{ }^{+}, \mathrm{NO}_{3}{ }^{-}$, $\mathrm{H}_{2} \mathrm{PO}_{4}{ }^{-}$and $\mathrm{SO}_{4}{ }^{-}$with high concentrations (Mastalerz 1977). Therefore, the drainage of nutrient solutions has high concentration of nutrient ion, and need to reuse for reducing loss of fertilizers and prevent the contamination of underground water sources by undesired ions, such as nitrate and dihydrogen phosphate. However, the recycling drainage solutions in hydroponic or artificial soil-based cultures has risk of an exponential increase in pathogenic bacteria concentrations, such as agrobacterium, powdery mildew, and Fusarium wilt. Therefore, the pathogenic fungi and bacteria have to be inactivated in the drainage solution for reducing the infection risk of pathogenic fungi and bacteria in root environments (Zhang et al. 2017).

\subsubsection{Plasma exposure to cultivation mediums}

Non-thermal plasma (NTP) is a non-equilibrium ionized gas where electron temperature is much higher than temperatures of ions and ambient gas as described in chap. 4. Thus, the plasma stays barely above room temperature, in some cases a NTP can even be touched with hands while operating. Therefore, there is less thermodynamic damage to the reaction targets. NTP generally contains many chemically reactive species including reactive oxygen and nitrogen species (RONS; $\mathrm{O}_{3},{ }^{1} \mathrm{O}_{2}, \bullet \mathrm{OH}, \mathrm{NO}_{2}$, $\mathrm{N}_{2} \mathrm{O}, \mathrm{NO}, \mathrm{CO}_{2}, \mathrm{HNO}_{3}, \mathrm{HNO}_{2}$, etc.) (Graves 2012; Lules et al. 2014; Holubová et al. 2020). Those RONS directly stimulate plants or react with surrounding media to 
trigger further complex reactions. In the past, generation of NTP required complicated and expensive equipment because it had to be generated in low-pressure conditions where ionization of gas particles is easier. The development of NTP at atmospheric pressure in recent years has increased the options for where it can be utilized and allowed for innovative applications.

Recently, NTP has found utility in biological fields such as plasma medicine and plasma agriculture (Graves 2012; Puač et al. 2018). Air molecules can be dissociated into atomic oxygen and nitrogen using NTP. Therefore, the dissociated oxygen and nitrogen species can directly be fixed into water, following that, both oxidized and reduced forms of nitrogen are produced in the water. It can also help in atomic nitrogen $\mathrm{N}$ transformation. Generally, atomic nitrogen is unstable in the soil system. In general, atomic nitrogen $\mathrm{N}$ is lost to the atmosphere by ammonia volatilization, $\mathrm{N}_{2} \mathrm{O} / \mathrm{N}_{2}$ denitrification and lost to groundwater by $\mathrm{NO}_{3}{ }^{-}$leaching. Therefore, the efficiency of $\mathrm{N}$ in fertilizers is low due to the above-mentioned loss process, and roughly $50 \%$ of fertilizers are not used by plants. This fact indicates that further $\mathrm{N}$ input will contribute crop yield slightly, in contrary, according to the influence of additional $\mathrm{N}$ input on the environment, strategies to reduce $\mathrm{N}$ waste need to be added. Here, NTP has possibilities for $\mathrm{NO}_{3}{ }^{-}$and $\mathrm{NH}_{3}$ production through oxidation and reduction processes of $\mathrm{N}_{2}$ after dissociation (Ranieri et al. 2020).

Plasma treated water (PTW), which is water irradiated by plasmas, has been used for promotion of seed germination, seedling growth and irrigating seeds, plants, and crops (Ranieri et al. 2020). Degree of acidity is an important factor in the stability of active species as $\mathrm{NO}_{3}{ }^{-}, \mathrm{NO}_{2}{ }^{-}$, and $\mathrm{H}_{2} \mathrm{O}_{2}$ during PTW storage. When water $\mathrm{pH}$ is close to 7.0, the produced active species concentrations decrease at initial stage, while the products remain stable at later stage of over time. While the tap water works as a buffer, long period treatment induces a decrease of $\mathrm{pH}$, in similar manner to deionized water. In cases of greater degree of acidity $(\mathrm{pH}<3.5), \mathrm{NO}_{2}{ }^{-}$is not stable. When $\mathrm{NO}_{2}{ }^{-}$concentration is on the same order of magnitude of $\mathrm{NO}_{3}{ }^{-}, \mathrm{NO}_{3}{ }^{-}$ concentration increases rapidly as follows.

$\mathrm{NO}_{2}^{-}+3 \mathrm{H}^{+}->2 \mathrm{NO}_{(\mathrm{aq})}+\mathrm{NO}_{3}^{-}+\mathrm{H}_{3} \mathrm{O}\left(\right.$ or $\left.\mathrm{H}_{2} \mathrm{O}+\mathrm{H}^{+}\right)$.

When both $\mathrm{NO}_{2}{ }^{-}$and $\mathrm{H}_{2} \mathrm{O}_{2}$ are included in PTW, peroxynitrite reactions have to also be considered as follows (Verlackt et al. 2018).

$\mathrm{NO}_{2}^{-}+\mathrm{H}_{2} \mathrm{O}_{2}+\mathrm{H}^{+}->\mathrm{ONOOH}+\mathrm{H}_{2} \mathrm{O}, \mathrm{ONOOH}->\mathrm{OH}+\mathrm{NO}_{2}$.

Reactivity of peroxynitrite induces $\mathrm{pH}$ drops, which contributes $\mathrm{NO}_{2}{ }^{-}$decay.

Other important issue is that PTW has a potential to cause physical destruction of bacteria or to induce physiological cell death as apoptosis. The interaction between RONS in PTW and peptidoglycan in cell wall causes disruption of the peptidoglycan. This disruption results physical destruction of the cell membrane, which causes cell content release, or cell death due to oxidation stress by RONS, which are penetrated into the cell through pores in cell membrane (Graves 2012; Holubová et al. 2020).

NTP also has the potential to induce adaptive response to abiotic stress. In nature of plant growth environment, the plants are commonly exposed by many kinds of stress such as UV irradiation, temperature change, chemically reactive gases, physical force, etc. Inside the plant organism, ROS are also produced naturally in the cells, mainly in chloroplasts from photosynthetic tissues and in mitochondria from 
non-photosynthetic tissues according to the electron transport processes. In common, ROS in the plant organism works as oxidative stress, which causes the restriction of plant growth, because ROS gives damage to cell membranes, proteins, and nucleic acids, and distorting redox homeostasis. Therefore, plants generally induce antioxidant mechanisms to scavenge ROS for keeping ROS in adequate concentration. In contrary, ROS contributes plant growth due to work as signaling molecules in plants. For example, ROS production affects auxin homeostasis and flux, which can ultimately affect plant growth, because auxin contributes plant growth and development worked as phytohormone (Ranieri et al. 2020).

\subsubsection{Effects of plasma-treated solutions on plant growth-hydroponics}

Hydroponics are widely used as crop cultivations. In hydroponic, the hydroponic solutions that contain dissolved fertilizer are used as the main source of nutrients for plant growth. In the plant cultivations, $\mathrm{N}$ fertilizers containing $\mathrm{N}$ as $\mathrm{NO}_{3}{ }^{-}$and $\mathrm{NH}_{4}{ }^{+}$ are mainly employed. As stated above, RONS can be generated by plasma, which plays an important role in $\mathrm{N}$ uptake in the culture medium of plants. In general, plants take $\mathrm{N}$ from the root plasma membrane in the form of $\mathrm{NO}_{3}{ }^{-}$and $\mathrm{NH}_{4}{ }^{+}$, in which $\mathrm{NO}_{3}{ }^{-}$is a major source for $\mathrm{N}$ in case of most plants. In hydroponic cultivation, $\mathrm{pH}$ is also an important parameter. The $\mathrm{pH}$ value generally changes in range from 5.0 to 6.0 in the growing period due to variations in the uptake of anions and cations. Uptake of cations such as $\mathrm{NH}_{4}{ }^{+}$is related to the release of $\mathrm{H}_{3} \mathrm{O}^{+}$, and then, the $\mathrm{pH}$ value decreases. Uptake of anions such as $\mathrm{NO}_{3}{ }^{-}$causes the release of $\mathrm{OH}^{-}$ by the plant, and then the $\mathrm{pH}$ value increases. Electrical conductivity (EC) is another important parameter in hydroponics, and is used to estimate nutrient concentration. A reasonable EC range is 1.6-3.0 mS/cm (Ranieri et al. 2020).

Many researchers reported about plant growth promotion by plasma exposure to solution used as cultivation bed. Takaki et al. reported the growth enhancement by plasma irradiation into the circulated water using a magnetic compression pulsed power generator. Brassica rapa var. perviridis was cultivated using drainage water in pots filled with artificial soil. The drainage water was exposed by plasmas produced in air bubbles injected into the drainage water of plant cultivation bed pots. The growth rate increased significantly when drainage water was irradiated with plasma, as shown in Fig. 58 (Takaki et al. 2013). The growth rate increased with plasma irradiation time, as shown in Fig. 59 (Takaki et al. 2013). It was also confirmed from chlorophyll content analysis that nitrogen concentration of chloroplast in leaves increased by the plasma irradiation to the water. The bacteria in the drainage water were also confirmed to be inactivated by water treatment with plasma. After a 28-day plant cultivation period, the concentrations of nitrate nitrogen $\left(\mathrm{NO}_{2}{ }^{-}\right)$ and nitrous nitrogen $\left(\mathrm{NO}_{3}{ }^{-}\right)$were not detected in non-irradiated drainage water, while the concentrations were obtained from the irradiated water and increases with plasma exposure duration. The plasma-treated water was acidified with $\mathrm{HNO}_{2}$ and $\mathrm{HNO}_{3}$ as $\mathrm{HNO}_{2(\mathrm{aq})}$ and $\mathrm{HNO}_{3(\mathrm{aq})}$ because Henry constants of $\mathrm{HNO}_{2}$ and $\mathrm{HNO}_{3}$ were larger than those of other species containing in the plasma in air bubbles. The nitrogen concentration of chloroplast in leaves at 28-day cultivation and the leaves length were also confirmed to be improved. The results indicates that the plant growth 


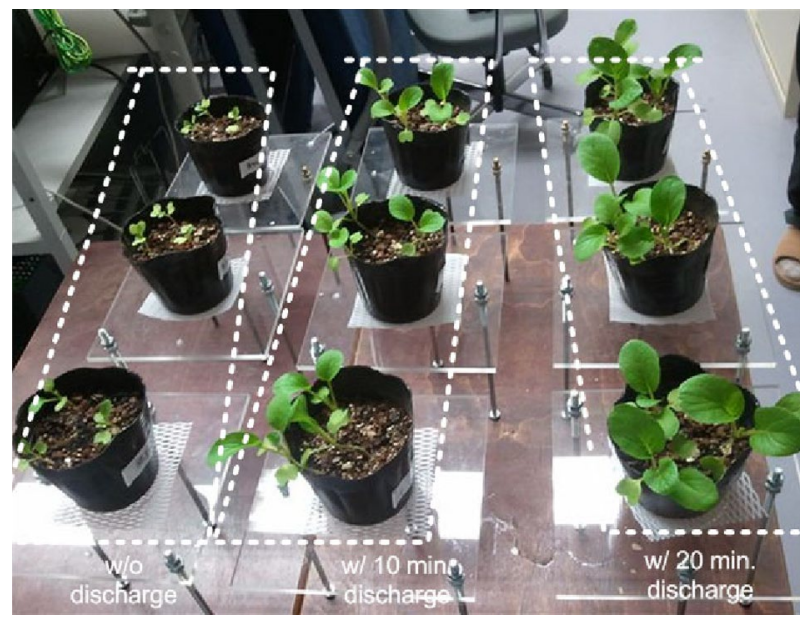

Fig. 58 Photograph of Brassica rapa var. perviridis cultivated for 28 days at a w/o plasma and with $\mathbf{b}$ $10 \mathrm{~min}$ or $\mathbf{c} 20 \mathrm{~min}$ irradiation per day (Takaki et al. 2013)
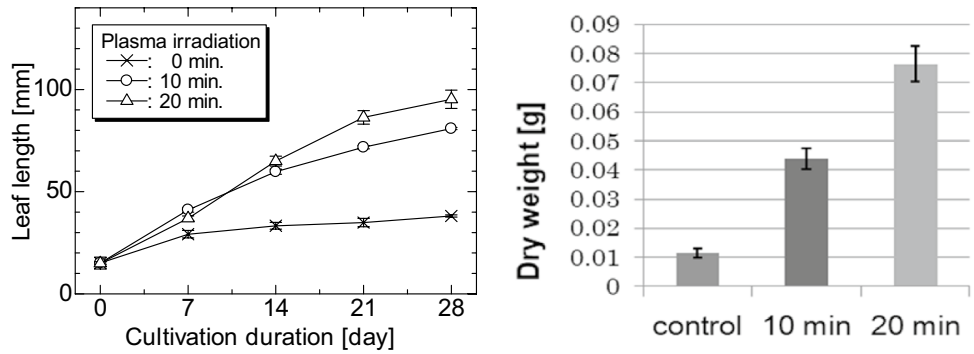

Fig. 59 Time history in leaf length (Left) and dried weight of cropped Brassica rapa var. perviridis after 28-day cultivation (right), for various plasma irradiation times (Takaki et al. 2013)

enhancement by plasma exposure to water supplied to bed soil is mainly caused by $\mathrm{NO}_{3}{ }^{-}$(aq) produced by plasma (Takaki et al. 2013).

Park et al. reported effects of plasma-treated water on seed germination, plant growth rates, and nutritional values for various plants using three types of plasmas (thermal spark discharge, gliding arc discharge, and transferred arc discharge). Water treated by the non-thermal gliding arc showed lower acidity (in $\mathrm{pH}$ ), and the generation of a large number of oxidizing species such as $\mathrm{H}_{2} \mathrm{O}_{2}$. Gliding arc dischargetreated water also showed large acidification (low $\mathrm{pH}$ ), however, and contained many kinds of RNSs such as $\mathrm{NO}, \mathrm{NO}_{2}{ }^{-}$and $\mathrm{NO}_{3}{ }^{-}$with high concentration. Spark discharge-treated water showed neutral or higher $\mathrm{pH}$, and also contained RNSs with high concentration (Park et al. 2013). The effect of the plasma treatments on plant growth were evaluated for various plants of watermelon (Citrullus lanatus), zinnia (Zinnia peruviana), alfalfa (Medicago sativa), polebeans (Phaseolus coccineus), and 
shade champ grass. The effects on growth enhancement depended on the discharge plasma type (Park et al. 2013).

Yemeli et al. investigated the effects of plasma-activated water (PAW; the meaning is identical to the above-mentioned PTW) generated by two sources of atmospheric air plasma on seedlings of maize (Zea mays L. var Saccharata) and barley (Hordeum vulgare L.). For plant growth, the length of barley plants with PAW increased by several percentage points compared to the control. The fresh weight of barley plants increased by several $10 \mathrm{~s}$ of percentage points when cultivated with PAW. Analytical results for RONS in PAW showed increases in concentrations of $\mathrm{H}_{2} \mathrm{O}_{2}, \mathrm{NO}_{2}{ }^{-}$, and $\mathrm{NO}_{3}{ }^{-}$compared to a tap water control. The total soluble proteins in the above-ground plants increased by about $20 \%$ both plants of barley and maize due to nitrates in the water solution. PAW induced active change of antioxidant enzymes of plants. Activity of superoxide dismutase (SOD) decreased by PAW for both plants. Guaiacol peroxidase (G-POX) activity increased in barley and slightly decreased in maize. Catalase (CAT) activity increased in both plants. Plant growth enhancement of barley using PAW was not accompanied by any DNA damage (Ndiffo Yemeli et al. 2020).

Hashizume et al. investigated the effects of non-thermal plasma during rice cultivation in paddy field on rice plant growth using two different approaches: direct plasma irradiation and Ringer's lactate solution (PAL) used as solution. During the early vegetative period, after irradiation for $30 \mathrm{~s}$, the weight of panicles and culms increased along with the number of panicles. In addition, plant height, main stem growth, and panicle weight increased by 5 min plasma irradiation. The harvest yield of brown rice increased in both irradiations of direct and PAL with increase rate of 12 and $15 \%$, respectively. In the metabolism of the rice plant, cytokinin is particularly important plant hormone for panicle differentiation and grain yield. The gene expression related cytokinin activity is also affected in stress conditions. Therefore, it is estimated as the reactive species induce cell division in the shoot apical meristem by cytokinin pathway (Hashizume et al. 2020).

The plasma irradiation can be used for improving growth of plants through inactivation of pathogenic bacteria in the cultivation bed. Okumura et al. reported inactivation of $R$. solanacearum using pulse plasmas in a tomato hydroponic. The pulsed plasmas were produced in air bubbles in the liquid fertilizer. The pulsed discharge was generated by MPC pulsed power generator. The number of $R$. solanacearum was analyzed using colony counting method. The results showed that $R$. solanacearum in the liquid fertilizer decreased from $10^{7}$ to $10^{2} \mathrm{CFU} / \mathrm{mL}$ by plasma irradiation to the water. Infection rate by $R$. solanacearum of tomato seedlings decreased with plasma treatment, while the infected positive controls all wilted and died, as shown in Fig. 60 (Okumura et al. 2016).

\subsubsection{Effect of plasma treatment on plant growth-soil}

In nature, acidity $\mathrm{pH}$ of soil is generally in range from 4.0 to 8.0. For crop plants cultivation, the $\mathrm{pH}$ of soil is modified because the optimal range for plants is from 5.5 to 6.5 . If $\mathrm{pH}$ of soils is $\mathrm{pH}<5.5$, the acidity is too large for plant growth, which has risk for exacerbating aluminum and manganese toxicity. The nitrogen $(\mathrm{N})$-based 

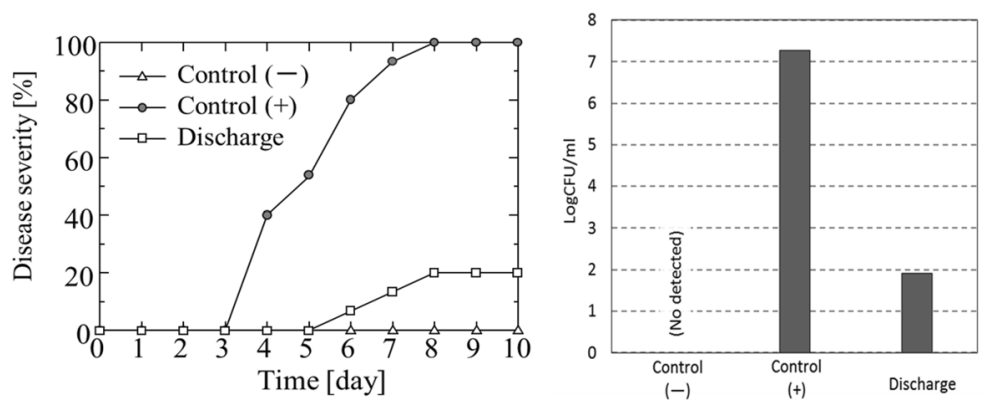

Fig. 60 Disease severity of tomato seedlings (Left) and number of colonies by $R$. solanacearum (right) in the liquid fertilizer. Control (-): negative, Control (+): positive control, and Discharge: discharge plasma treatment (Okumura et al. 2016)

fertilizers generally acidify soil by producing $\mathrm{H}^{+}$through oxidation process of $\mathrm{NH}_{4}^{+}$ to $\mathrm{NO}_{3}{ }^{-}$. The plasma-based fertilization accompanies to acidify the soil by released $\mathrm{H}^{+}$from $\mathrm{NO}_{3}{ }^{-}$production process, in same manner to $\mathrm{N}$ fertilizers. Therefore, plasma treatment of bed soil through $\mathrm{N}$ production requires the same soil acidity management as traditional fertilizers (Ranieri et al. 2020).

Soil microbes are a category of soil organisms that are important for health and resilience of soil. Microbials in vicinity of the plant roots are responsible for nitrogen cycling, which is significantly important in plant growth and soil fertility. Nitrogen $(\mathrm{N})$ cycling is microbial transformations including regulation of available $\mathrm{N}$ biologically in soil, and exchanging $\mathrm{N}$ with the atmosphere through $\mathrm{N}_{2}$ fixation and denitrification processes, as shown in Fig. 61a (Ranieri et al. 2020).

In addition to $\mathrm{N}$ production as $\mathrm{N}_{2}$ fixation, direct plasma irradiation or PTW to the soil affects microbial viability by ROS and RNS produced by plasma. ROS and

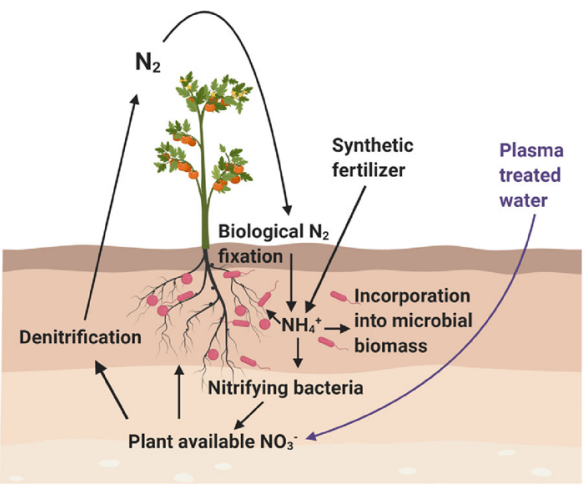

(a)

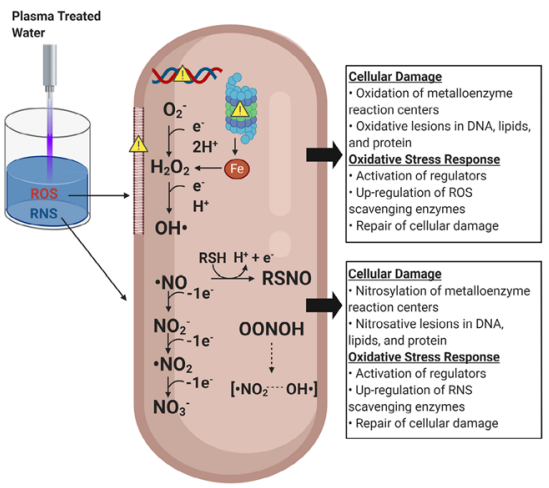

(b)

Fig. 61 Impact of plasma treated water (PTW) for plants on soil and microbiome. a Microbial nitrogen cycle in agricultural soils with PTW as additional nitrate source, $\mathbf{b}$ molecular responses of bacteria by exposure to ROS and RNS. ( Reproduced with permission from Ranieri et al. 2020) 
RNS can affect cell viability and microbial cell function through the damage of lipids in cell membranes, cytoplasmic proteins and DNA. Moreover, RNS induces inhibit of cellular respiration, as the result, reducing cellular energy generation. RNS also reduces DNA replication owing to the inactivation of zinc metalloproteins. Both ROS and RNS enhances ROS toxicity by formation of reactive hydroxyl radical $(\mathrm{OH} \cdot)$ through Fenton reaction by iron of iron-sulfur-containing dehydratases. ROS and RNS acting individually or synergistically induce a broad antimicrobial activity, including bacteria, fungi and parasites, as shown in Fig. 61b (Ranieri et al. 2020).

Ozone $\left(\mathrm{O}_{3}\right)$ is commercially available disinfectant and also is very effective at inactivating bacteria and viruses, and decomposing odor chemicals. $\mathrm{O}_{3}$ has a short residence time in water and soil, and either decomposes to diatomic oxygen or consumes via reactions with other compounds as oxidization processes. Ebihara et al. reported the gaseous $\mathrm{O}_{3}$ sterilization for soil and biomedical prevention for infectious diseases. Bacteria concentration in the soil was reduced with $80 \%$ of reduction rate by ozone treatment with 20 min treatment duration and $20 \mathrm{gO}_{3} / \mathrm{m}^{3}$ ozone concentration. The oxidization of DNA by $\mathrm{O}_{3}$ irradiation is considered as dominant mechanism for inactivation of bacteria in agricultural soil. Experiment results showed that DNA structures were destroyed through treatment with ozone $\left(100 \mathrm{gO}_{3}\right.$, $0.5 \mathrm{~L} / \mathrm{min}, 20 \mathrm{~min}$ ). Ozone is believed to break up nucleobase hydrogen bonds (thymine-adenine pairs, cytosine-guanine pairs) in DNA (Ebihara et al. 2012). Mitsugi et al. used surface barrier discharge ozonizer for soil treatment as both a pesticide and a fertilizer. It was confirmed that $\mathrm{O}_{3}$ was effective for inactivation of nematodes in the soil. $\mathrm{O}_{3}$ molecules were taken into the nematodes by diffusion through their epidermis. The taken $\mathrm{O}_{3}$ causes oxidative damage to cellular lipids, proteins, and nucleic acids. The effect of soil treatment by $\mathrm{O}_{3}$ irradiation on growth of radish planted in the ridges in a greenhouse was also evaluated. The all-radish growth indices (germination rate, fruition rate, worm-eaten rate, leaf length, stem length, fruit length, and total harvest weight) were improved by $\mathrm{O}_{3}$ soil treatment (Mitsugi et al. 2016).

Plasma activated water (PAW) was not only used for direct inactivation of pathogenic bacteria and seedling growth enhancement, but also utilized to enhance the defense from the infection of pathogenic disease. Perez et al. reported that the PAW worked as resistance inducer against bacterial leaf spot of tomato. PAW was produced using dielectric barrier discharge (DBD). PAW did not show direct antimicrobial activity, but it enhanced the tomato plants defenses. It was effective in reducing the disease severity by giving relative protections before the inoculation (Perez et al. 2019). In addition, Laurita et al. reported the effect of PAW for induction of resistance and agronomic performance in viticulture. PAW was produced using two different cold plasmas; DBD and corona discharge. The DBD was driven by nanosecond pulse generator. The corona was generated by commercial millisecond pulse generator. The results showed the capability of PAW to enhance plant defense mechanisms and confirm its ability to improve the health status of the treated plants (Laurita et al. 2021). 


\subsection{Pulsed electric field stimuli for fruiting body formation}

Electrical stimuli can be used for promotion of plant growth mode change from vegetative to reproductive. Mushrooms are mainly belonged to basidiomycetous fungi and some are ascomycetous fungi, thereby, mushrooms commonly form spore at reproductive growth phase (Lull et al. 2005). Proper control of applied electrical energy can cause physical stimulus to the mushroom mycelium in the cultivation environment, resulting in promotion of fruiting body formation for increasing harvest yield (Takaki et al. 2018). This section describes the effect of electrical stimuli, which is applying electrical energy to mushroom spores or mycelium through the cultivating medium, on fruit body formation of mushrooms.

\subsubsection{Mushroom cultivation and stimuli for fruiting body development}

The life cycle of mushroom is shown in Fig. 62 (Lull et al. 2005). Mushroomforming fungi precepts by sensing several environmental factors for its growth. In general, nutrient, temperature and light conditions are critical factors for induction of fruit body formation. Although higher nitrogen and carbon sources in the media is suitable for vegetative growth, the higher nitrogen and carbon sources generally suppress induction of fruit body formation in many mushroom-forming fungi. The induction of fruit body formation is triggered by low nitrogen and carbon concentrations. Low temperature or differential of temperature to decrease is another critical factor for the induction. Fungal response to starvation and low temperature is related to production of spores (seeds) for the next generation.

Critical factors for induction of fruit body formation is one of important issue in mushroom cultivation (Thakur 2020). In common, environmental factors for the induction can be classified into two groups: physiological and physical. For example, physiological factor includes conditions of gaseous, nutrient, and hormones. Vibration or striking of bed medium, wounding the mycelium are work as physical stimulation. Light is one of the crucial factors for induction of fruit body formation. Especially, blue light is most effective wavelength to trigger the formation of fruit body of mushrooms such as in Lentinula edodes (L. edodes; shiitake mushroom). Low temperature or temperature deviation is other critical factors for promotion of fruit body formation. Especially, down shift of temperature effectively works as stimuli for fruit body formation. For example, Flammulina velutipes ( $F$. velutipes) is triggered for fruit body formation by temperature down from 23 to $16{ }^{\circ} \mathrm{C}$ (Sakamoto 2018). Wood decay fungi such as L. edodes, F. velutipes, Pholiota nameko are major species for commercially cultivating mushrooms. In cultivation of wood decay fungi, degree of wood decay is closely related to induction of fruit body formation.

\subsubsection{History of electrical stimuli for mushroom fruiting body development}

Pulse electric fields and artificial lightning using impulse high-voltage generator have been used to improve yielding rate of edible mushroom by some research groups from 1987 (Takaki 2021). Effect of high-voltage direct stimulation to 


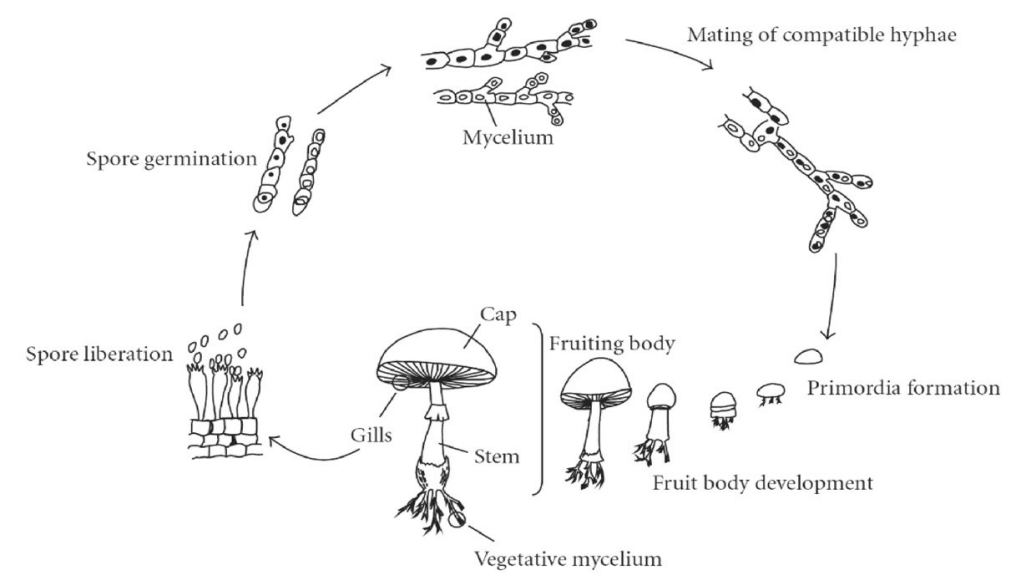

Fig. 62 Diagrammatic representation of mushroom life cycle. ( Reproduced with permission from Lull et al. 2005)

bed-log of L. edodes (Shiitake mushroom) on promotion of fruit body formation was confirmed by some researchers (Takaki et al. 2014). This effect was also confirmed in sawdust-based cultivation of $L$. edodes of promotion of fruit body formation (Kudo et al. 1999; Ohga et al. 2001). The increase of yielding rate of fruit body was reported as $170 \%$ with direct electrical stimulation in sawdust cultivation (Ohga and Iida 2001). The increase of yielding rate of fruit body by the high-voltage stimulation was also confirmed in many species of edible mushrooms of Grifola frondosa, P. microspora, F. velutipes, Hypsizygus marmoreus, P. ostreatus, P. eryngii, P. abalones and Agrocybe cylindracea (Ohga et al. 2004; Takaki et al. 2004). The increase rate of fruit body harvesting by the high-voltage stimulation was obtained as $130-180 \%$ in comparison with controls (Ohga et al. 2004) The high-voltage stimulation was also used for induction of fruit body of ectomycorrhizal fungi such as Laccaria laccata and Tricholoma matsutake (Takaki et al. 2004; Ohga and Iida 2001; Islam and Ohga 2012).

In early stage of the study, large-scale impulse generators was employed for generation of artificial lightning for stimuli on induction of fruit body formation, as shown in Fig. 63 (Idei et al. 1988). The conventional impulse generator (IG) for test tool in insulation devices are commonly used in power distribution system. The commercial IG consists of 10-20 capacitors, 10-20 gap switches and damping resistors as shown in Fig. 63. The capacitors are connected in parallel at charging phase as mentioned in Sect. 3.3. The output voltage is multiplied by changing the connection of the capacitors from parallel to series using gap switches after charging up the capacitors. Output voltage of IG is commonly in range from $250 \mathrm{kV}$ to $1 \mathrm{MV}$ (Kuffel et al. 2000). The output voltage waveforms including rise and fall times are controlled by parameters of discrete elements in microsecond-order simulated as an artificial lightning stroke voltage. Typical output voltage waveform applied to the mushroom bed-log is shown in Fig. 64 (Kaneko et al. 1987). The waveform of Fig. 64 shows the peak voltage of $288 \mathrm{kV}$ 
Fig. 63 Photograph of impulse generator for stimuli of Shiitake mushroom cultivation bed-log (Idei et al. 1988)
Fig. 64. $288 \mathrm{kV}$ output voltage of an impulse generator. $X: 1 \mu \mathrm{s} /$ div., $Y$ : voltage $(50 \mathrm{kV} / \mathrm{div}$. $)$ (Kaneko et al. 1987)
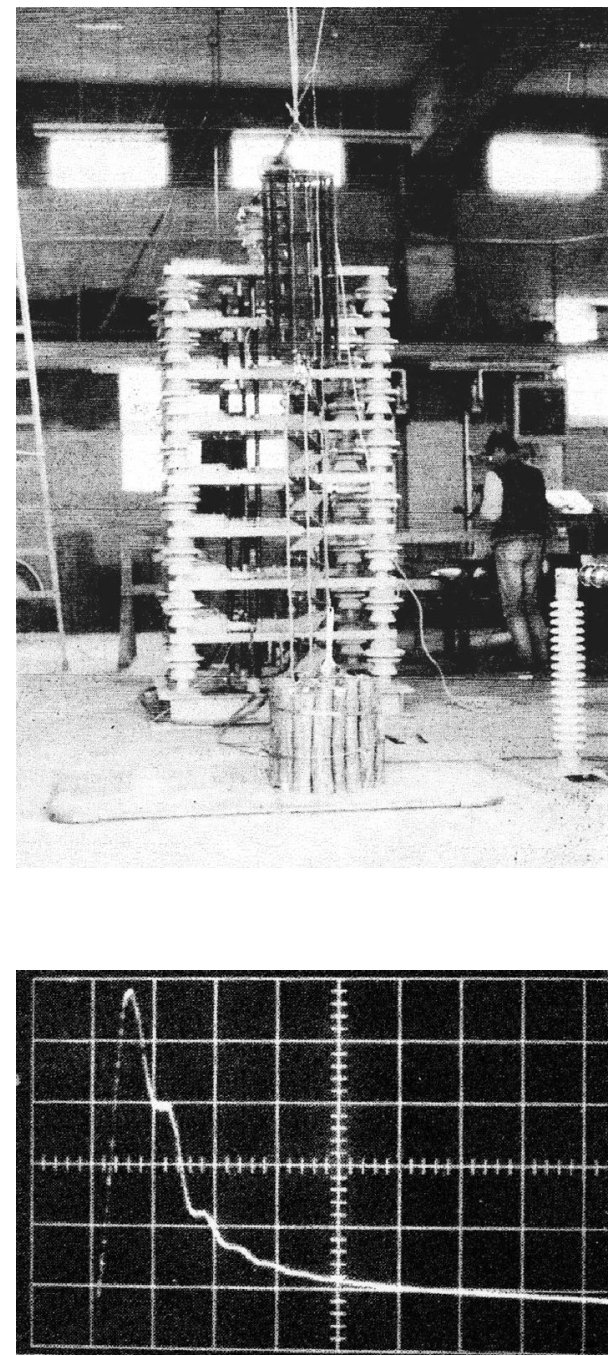

with rise time close to $0.5 \mu$ s. The pulsed high-voltage of IG output was applied to the bed-logs (Konara oak; Quercus serrata) as shown in Fig. 65 (Idei et al. 1988). The length of bed-logs was $1 \mathrm{~m}$, which was same dimension commonly used in log-based cultivation of $L$. edodes. The 5-9 bed-logs were bundled or connected in parallel and was connected to high-voltage electrode at top side and other end grounded. The high-voltage stimulation was carried out in phase of vegetative growth. After high-voltage stimulation, the bed-logs are cultivated in the house for fruit body formation. Yielding rates of the fruit body cropped from the bed-logs were obtained for each stimulation condition.

Tables 2 and 3 show the effects of high-voltage stimulation on harvest yield of $L$. edodes fruit body for various voltage strengths (Kaneko et al. 1987; Takaki 2021). 

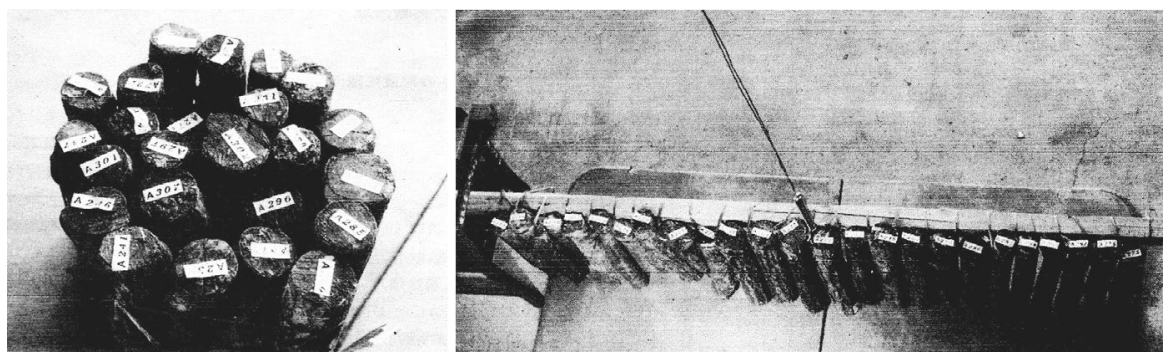

Fig. 65 Photographs of setup of bed-logs for high-voltage stimulation by commercial impulse generator (Idei et al. 1988)

Table 2. Yielding of fruit body of L. edodes from bed-logs for different applied voltage used in high-voltage stimulation without submergence treatment (Kaneko et al. 1987; Takaki 2021)

\begin{tabular}{llll}
\hline Exp. group & $\begin{array}{l}\text { Number of exp. } \\
\text { bed-logs }\end{array}$ & $\begin{array}{l}\text { Fruit-body yield (per } \\
1 \mathrm{~m}^{3} \text { of wood) } \\
\text { Number }\end{array}$ & Dry wt (g) \\
\hline $144 \mathrm{kV}$ & 24 & 505.3 & 1337.0 \\
$288 \mathrm{kV}$ & 24 & 770.1 & 2171.4 \\
$576 \mathrm{kV}$ & 24 & 121.6 & 558.4 \\
Contd & 24 & 16.9 & 55.2 \\
\hline
\end{tabular}

Bed-log age: 38 months after inoculation (Yakult haru 2). Water content of bed-logs: $38.9 \%$ (mean value of six samples). All exp. groups had $34 \mathrm{~mm}$ rainfall in a week after discharge

Number and total dry weight of harvested fruit bodies increase by applying high voltage as stimulation for promotion of fruit body formation. Both without and with submergence treatment cases showed that the yielding rate of fruit body harvest increase by applying high-voltage pulse as stimulation. However, the dependency of applied voltage strength on yield of fruit body without submergence treatment has slightly different tendency from those with submergence treatment. Yielding rate at $288 \mathrm{kV}$ of applied voltage strength is much larger than controls with improving rate of $393 \%$ in case without submergence treatment as shown in Table 2. The yielding rate at $288 \mathrm{kV}$ is larger than control with improving rate of $250 \%$ without submergence treatment, as shown in Table 3. When the applied voltage is too high compared with the optimum condition, the mycelium is physically damaged strongly, thereby the stimulation suppress the fruit body formation. In addition, the bed-logs are also damaged by shockwave through gas thermal kinetics caused by pulsed arc discharge with high current (Idei et al. 1988).

Idei et al. (1988) reported the distributions of harvested number of fruit bodies for each bed-log under same stimulation condition with Table 1, as shown in Fig. 66. In case without high-voltage stimulation (control), the fruit body did not appear on 20 bed-logs $(83 \%)$, and one fruit body appeared on 4 bed-log $(17 \%)$. The fruit bodies were harvested from 21 bed-logs (except 3 bed-logs; 12\%) at $288 \mathrm{kV}$ of voltage strength used as stimulation. This decrease of number of bed-log without L. edodes 
Table 3. Yielding of fruit body of L. edodes from bed-logs for different applied voltage used in high-voltage stimulation with submergence treatment (Kaneko et al. 1987; Takaki 2021)

\begin{tabular}{llll}
\hline Exp. group & $\begin{array}{l}\text { Number of exp. } \\
\text { bed-logs }\end{array}$ & $\begin{array}{l}\text { Fruit-body yield (per } \\
1 \mathrm{~m}^{3} \text { of wood) } \\
\text { Number }\end{array}$ & Dry wt (g) \\
\hline $288 \mathrm{kV}$ & 21 & 650.8 & 2100.0 \\
$576 \mathrm{kV}$ & 21 & 485.8 & 1648.9 \\
$720 \mathrm{kV}$ & 21 & 453.8 & 1427.4 \\
Cont & 21 & 276.2 & 840.6 \\
\hline
\end{tabular}

Bed-log age: 38 months after inoculation (Yakult haru 2). Water content of bed-logs: $42.3 \%$ (mean value of six samples)

fruit body mainly contributes to increase yield of mushroom harvesting by applying pulsed voltage produced by IG.

\subsubsection{Effect of high-voltage stimulation in bed-log cultivation}

Several kinds of edible mushrooms are cultivated artificially. Many of them are wood-rotting fungi and so mushrooms grow on dead trunks of wide leaf trees in the forest. Lentinus edodes (Shiitake) was localized wild in the southern part of Japan from long time ago and by the invention of wood-chip spawn by the late Dr. Mori, scientific artificial cultivation started at 1943. They are now cultivated in the forests by inoculated bed-logs of oak trees everywhere in Japan. In 2019, 88,031 tons of fresh Shiitake mushrooms are harvested in a year and 16,901 tons of them are used as dried Shiitake. Beside Shiitake, Flammulina velutipes (Enokitake), Pholiota glutinosa (Nameko), Pleurotus ostreatus (Hiratake), Glifola frondosa (Maitake), Agaricus bispous, Lyophyllum ulmarium (Shirotamogitake) and others are cultivated commercially. Most of them are cultivated by sawdust culture (bottle or bag culture) in air-conditioned rooms. Many good strains are selected from wild strains or cross breeding is carried out by several spawn makers. Now the cultivation of edible mushrooms has grown to a big business for the farmers. The total production of cultivated mushrooms in a year is 456,481 tons and the market price is about 226 billion Japanese yen.

The bed-log cultivation of mushroom is widely used in the forest and green house. In general, mushrooms such as shiitake are first inoculated into logs. After germination of spores inoculated in the log, the hyphae grow inside the logs over a period of more than 1 year. After the hypha grow in whole logs, the phase changes from hyphal-level vegetative growth to fruiting body (mushroom)-level reproductive growth triggered by some stimulations. At this time, mushroom farmers use some stimulations to promote a mushroom outbreak. At first, the bed-log is submerged for 1-2 days, followed by beating several times as physical stimulus. This stimulus causes physiological and biochemical changes in hyphae of mushroom owing to the temperature change by inundation and physical stimulus, inducing in a shift from vegetative to reproductive growths. However, the effects of both methods are highly dependent on the famer's experience, thereby the expected effects can often not be 

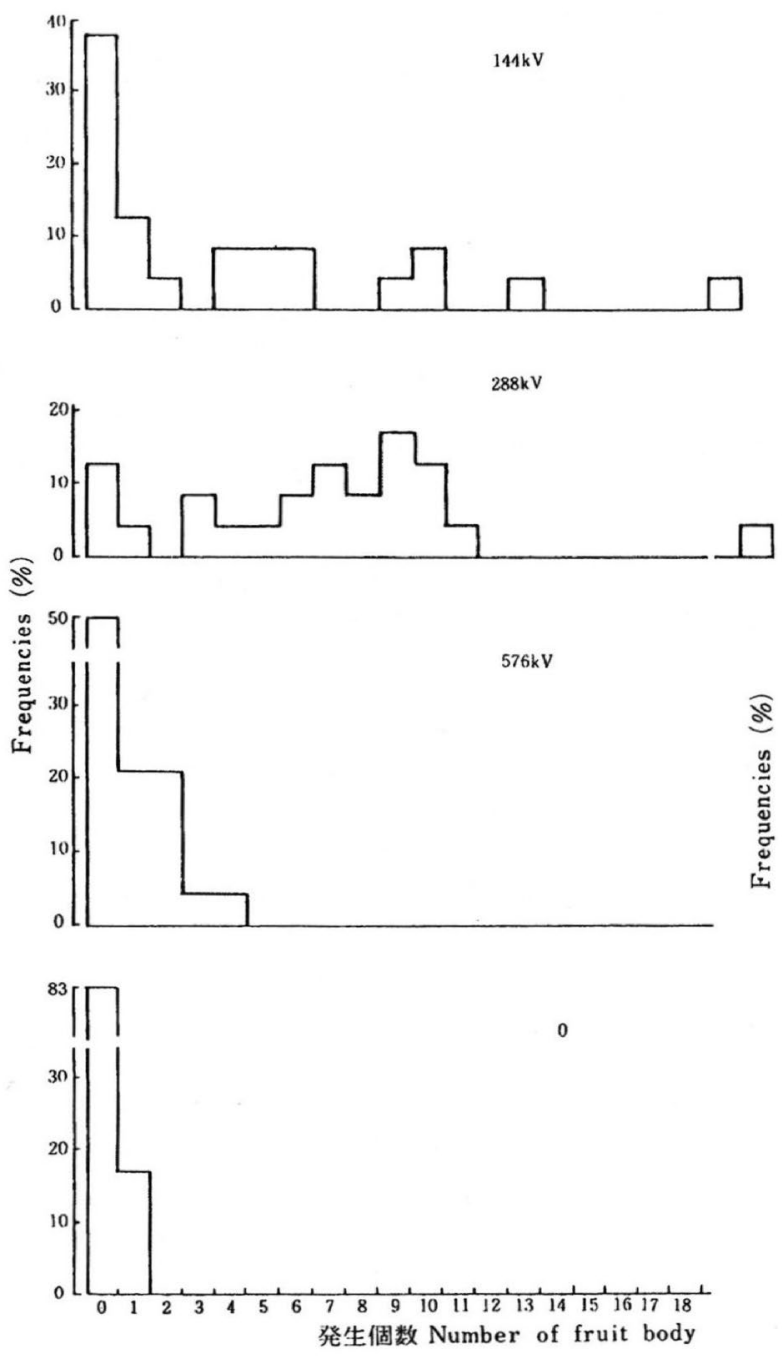

Fig. 66 Distribution of developed fruit body number for different strength of high-voltage stimulation to L. edodes cultivated bed-logs without water submerged treatment (Idei et al. 1988)

achieved. In addition, these processes are hard for elderly formers to maintain production levels.

Takaki et al. reported the effect of high-voltage stimulation to L. edodes bed$\log$ on harvest yield of fruit body in period of four flashes (two years), as shown in Fig. 67. Four stages Marx generator was used to generate high voltage with pulse width of several microseconds as shown in Fig. 13 (Takaki et al. 2014). The output voltage was changed from 50 to $125 \mathrm{kV}$ of strength and was applied to top of bed-log which has $0.9 \mathrm{~m}$ of length and approximately $1 \mathrm{~kW}$ of resistance. Number of bedlogs for one group was set to be fifteen. The yield of the fruit body was evaluated as 


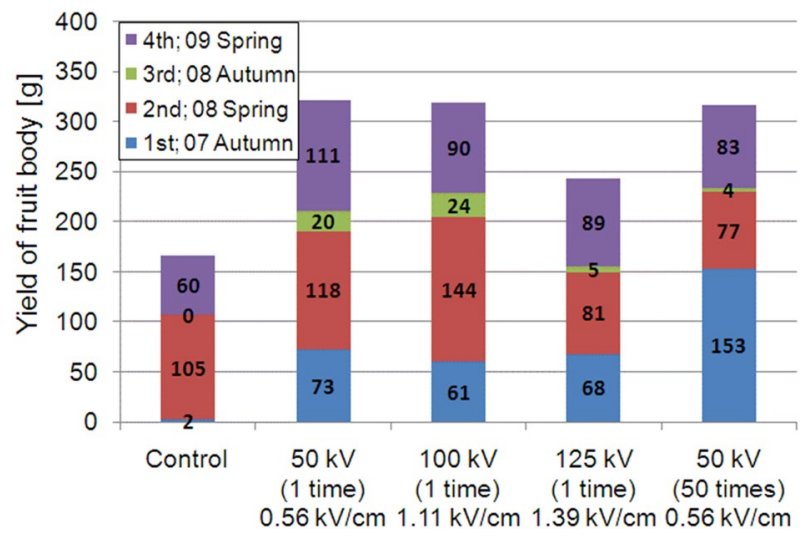

Fig. 67 Total weight of harvested fruit bodies of $L$. edodes for various electrical stimulation conditions during four flash seasons (Takaki et al. 2014)

total flesh weight harvested for each season for fruit body development. The results showed that the yield of the control group was only $2 \mathrm{~g}$ in the first harvest season (October 2007). In this case, the $30 \mathrm{~g}$ weight of fruit bodies was harvested from only one $\log$. The yield at first season increased to $73 \mathrm{~g}, 61$ and $68 \mathrm{~g}$ by applying voltage of 50,100 , and $125 \mathrm{kV}$, respectively. For $50 \mathrm{kV}$ voltage, the yield increased from 73 to $153 \mathrm{~g}$ with increasing number of high-voltage stimulation from 1 to 50 times. The total weight over four seasons also increased from 167 to 322, 319, and $243 \mathrm{~g}$ by applying high voltage at $50,100,125 \mathrm{kV}$, respectively. The optimum voltage strength for stimuli to induce fruit body formation can be estimated in range from 50 to $100 \mathrm{kV} / \mathrm{m}$ under the experimental condition (Takaki et al. 2014). The flesh weights of fruit bodies of $L$. edodes harvested from each bed-log at applying $50 \mathrm{kV}$ pulse voltage as stimuli for fruit body formation, as shown in Fig. 68. Figure 69 also shows the photographic image of the difference between harvesting yield with and without electrical stimulation using $50 \mathrm{kV}$ applying voltage. In this experiment, the autumn was chosen as season for evaluation of effect of high-voltage stimulation on promotion of fruit body formation, because the used strain of L. edodes was generally suitable for the spring harvest. The total fresh weight of harvested fruit bodies from all bed-logs were $1.09 \mathrm{~kg}(=73 \mathrm{~g} \times 15)$ and $2.29 \mathrm{~kg}(=153 \mathrm{~g} \times 15)$ for one time and 50 times stimulations by $50 \mathrm{kV}$ pulse, respectively. This result indicates the total yield of fruit bodies harvest can be improved by increasing electrical stimulation number. For the aspect of harvest from each bed-log, the maximum weights harvested from each bed-log were 300 and $320 \mathrm{~g}$ for one time and 50 times stimulations by $50 \mathrm{kV}$ pulse, respectively. Both values are similar. However, the number of bed-logs in which the fruit bodies are harvested are $8(53 \%)$ and $15(100 \%)$ for one time and 50 times stimulations by $50 \mathrm{kV}$ pulse, respectively. This result revels that the multi times stimulations by high voltage contributes to decrease the deviation in fruit body formation, resulting the improvement of harvest yield.

The pulse voltage applying works as stimulation for induction of fruit body formation. The effect of the electrical stimulation on the induction of fruit bodies were 


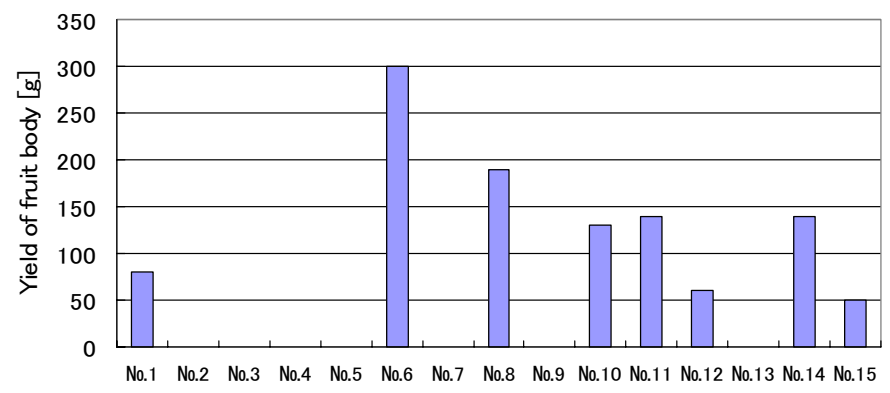

(a)

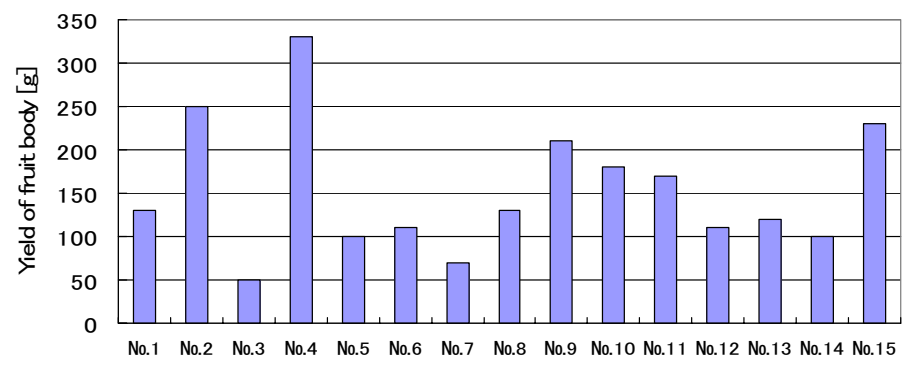

(b)

Fig. 68 Yield of harvested fruit bodies of L. edodes for each bed-log with $50 \mathrm{kV}$ applied voltage stimulation. No.1-No.15 indicate labels for each cultivation log. a One time stimulation; b 50 times stimulations (Takaki et al. 2014)

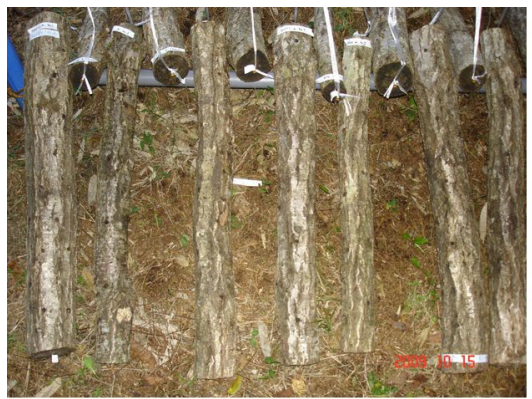

(a)

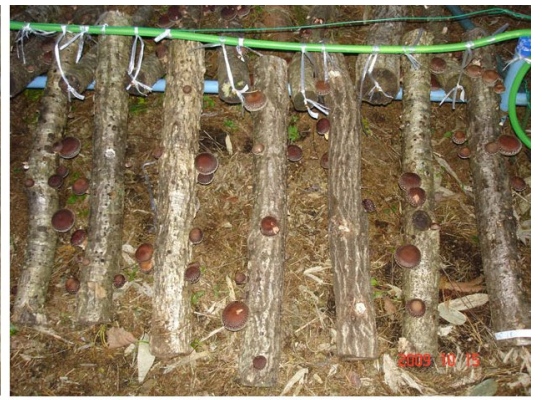

(b)

Fig. 69 Photographs for difference in the fruit body developing a without and $\mathbf{b}$ with $50 \mathrm{kV}$ high voltage stimulation

evaluated by timing of fruit body development from the stimulation, as shown in Fig. 70 (Takaki et al. 2014). The yield in Fig. 70 shows normalized value which is calculated as the accumulated yield from the day of stimulation to harvest divided by total crop weight for one harvesting season. The four-stage Marx generator was used to generate impulse high voltage of 50,100 and $125 \mathrm{kV}$ of strength. The normalized 


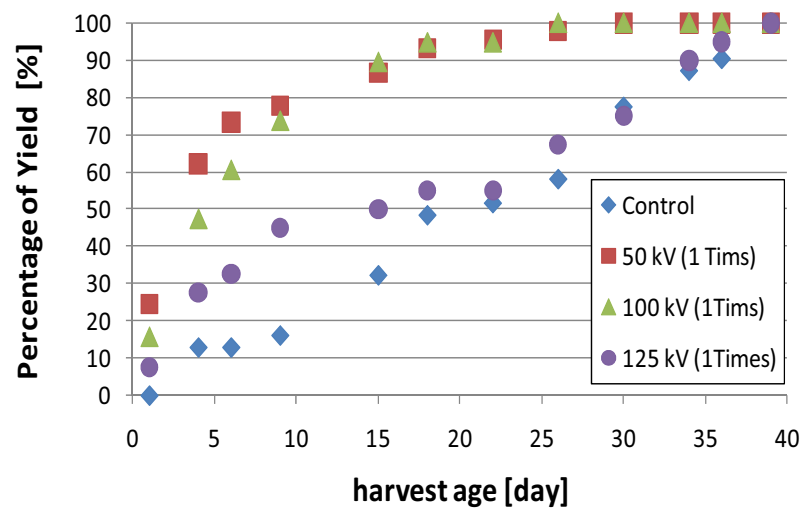

Fig. 70 Time-history of the accumulated fresh weight of harvested fruit bodies for various stimulation voltages. The accumulated weight is normalized by total weight harvested in one flash season (Takaki et al. 2014)

yield at 15 days from the day of first crop (almost 1 week after the stimulation) is approximately $50 \%$ of the total in control group. However, the crop weight during same period increases to $86 \%$ by applying high voltages of 50 and $100 \mathrm{kV}$ as stimulation for fruit body formation. This result revels that the high-voltage stimulation induces the fruit body development, resulting fruit bodies developing at similar duration.

Shimizu et al. reported the improvement of harvest yielding of mushroom ( $L$. edodes) by stimulation using shockwave generated by long-gap pulse arc discharge (Shimizu et al. 2020). The artificial lightning impulse voltage was generated by an impulse voltage generator (IG), was applied to rod type high-voltage electrode to generate long-gap discharge. The mushroom culturing bed-logs were located apart from the discharge rod. cultivation bed-log. The yield of shiitake (L. edodes) fruit bodies increased with almost double by shockwave irradiation to the bed-logs compared to that without the indirect lightning strikes. The number of fruit bodies harvested per unit volume $\left(1 \mathrm{~m}^{3}\right)$ increased from $69.2(2.47 \mathrm{~kg}$ in total harvesting weight) to $132.2(4.18 \mathrm{~kg}$ ) by applying indirect lightning strikes. The results indicate that an indirect lightning strikes also works as physical stimulation for fruit body induction, resulting the improvement of shiitake mushroom production.

\subsubsection{Effect of high-voltage stimuli in bed-sawdust cultivation}

Nowadays, many kinds of edible mushrooms are cultivated artificially. Many of them are wood-rotting fungi and are mainly cultivated using bed sawdust (bottle or bag culture) in air-conditioning rooms including green house. In general, mushrooms such as shiitake are firstly inoculated into sawdust bed. After germination of spore, the hyphae grow in the sawdust bed over a period of three months. After hyphae growing whole sawdust bed, the growth phase changes from hyphal-level vegetative growth to fruiting body (mushroom)-level reproductive growth. In this timing, mushroom famers generally treat the sawdust bed by submerged for one day, 
followed by beating several times as physical stimulus to induce a mushroom outbreak as same manner with log-based cultivation.

Effect of pulse electric field stimulation on promotion of fruit body formation in sawdust cultivation has been investigated by some researchers. For example, Takaki et al. reported the effect of pulse electric field stimulation of harvest yield of fruit bodies of L. decastes in sawdust-based cultivation, as shown in Fig. 71 (Takaki et al. 2014) and Fig. 72. The yield was evaluated by the average fresh weight of fruit body cropped per a block for two flush seasons. Cockcroft-Walton circuit (shown in Fig. 8) was used to apply high voltage of $30 \mathrm{kV}$ on the sawdust bed, which had $0.35 \mathrm{k} \Omega$ of impedance. The electric field inside of the sawdust bed was strengthened at top (the point of high voltage applying), and was ranged from 18 to $360 \mathrm{kV} / \mathrm{m}$ at $30 \mathrm{kV}$ of applied voltage strength (Takahashi et al. 2018). It was confirmed that the yields of fruit bodies from sawdust bed were improved by pulse electric field stimulation. The yield was also confirmed as positive relation to input energy of pulse electric field to the bed-block. L. decastes in the stimulation group grew faster than those in the control group as photographs shown in Fig. 72 (Takaki et al. 2014).

Norarat et al. reported that the harvesting yield was improved by two different kinds of stimuli: high-voltage and oxygen-rich fine bubble (FB) water. The effects of high-voltage stimulation and oxygen-rich FB water were confirmed on sawdust cultivated shiitake mushroom (L. edodes) in Thailand. The combination of high-voltage stimulation and $\mathrm{O}_{2}$-rich $\mathrm{FB}$ water significantly promoted the total weight of shiitake mushroom by 1.8 times compared with the control (Norarat et al. 2019).

\subsubsection{Morphological changes after electrical stimulation}

There are two groups (physiological and physical) of environmental factors for the induction of fruit body. The physiological factor includes conditions of gaseous,

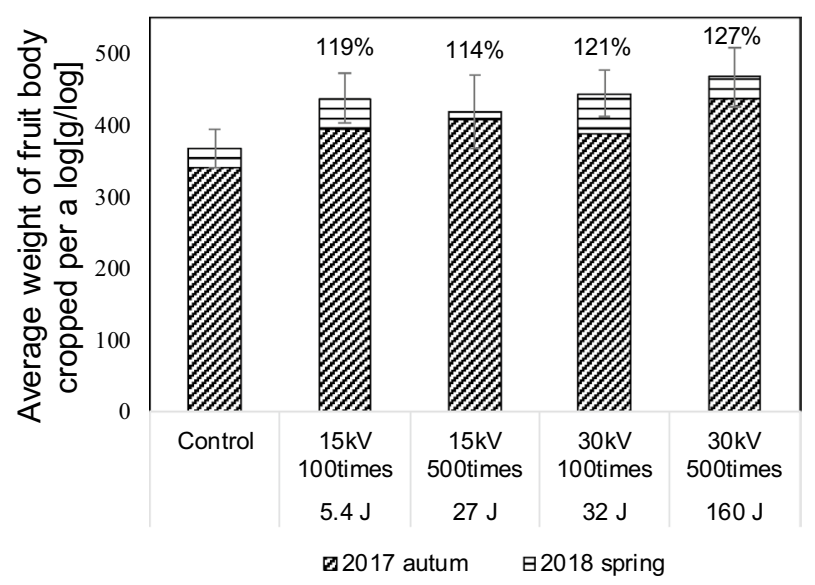

Fig. 71 Yield of fruit bodies of Lyophyllum decastes Sing. per a cultivation sawdust bed for 2 flush seasons for various conditions of pulse electric field stimulation (Takaki et al. 2014) 

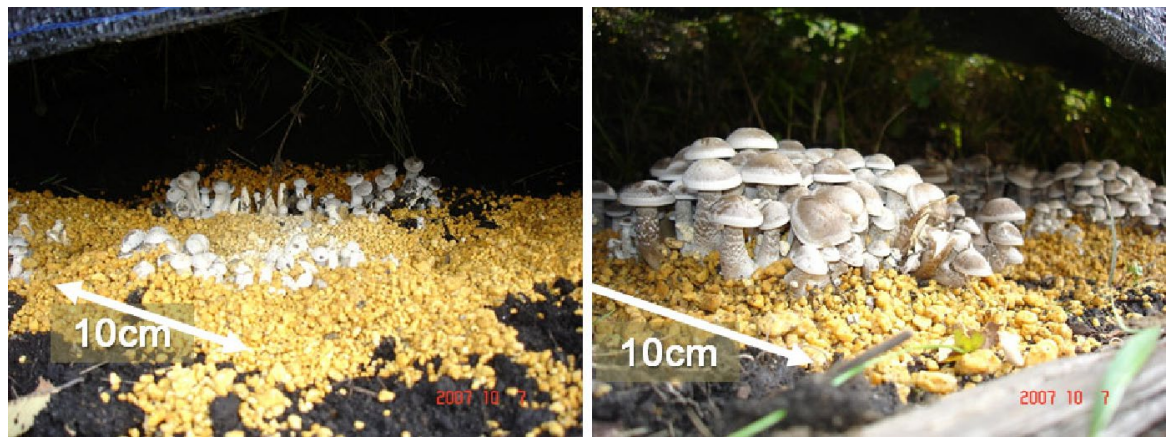

Fig. 72 Photographic images of developed fruit body of cultured Lyophyllum decastes Sing. without (left) and with (right) pulse electric field stimulation

nutrient and hormones. On the other hand, the physical factor includes striking bed medium and wounding the mycelium. The pulse voltage stimulation is considered as physical factor similar effects of striking bed medium and wounding the mycelium (Takaki 2021). Morphological changes of mushroom hypha after electrical stimulation were also reported using microscopic observation, as shown in Fig. 73 (Takaki 2021). In Fig. 73, figures (a) and (b) shows microscopic images of L. edodes hyphae distribution before (a, red) and after (b, blue) applying pulse electric fields. The hyphae were cultured on Potato Dextrose Agar (PDA). The pulse electric field was applied along the surface of PDA. Figure (c) shows a superimposed image of figures (a) and (b). Therefore, purple (red + blue) color indicates that hyphae retain the same position before and after applying pulse electric fields. In contrary, red- and bluecolored hyphae indicates the displaced hyphae. This displacements can be explained as the hyphae will be affected by Coulomb force $f=q E$; ( $q$ charge of hypha, $E$ electric field strength), resulting that the hyphae are accelerated towards the positive electrode according to the equation $f=m a$, where $m$ and $a$ are hypha mass and acceleration of the hypha, respectively. The application of electric pulses, resulting in hyphal displacement and sometimes damage, can be considered as a form of physical stress. Figure (d, e) shows scanning electron microscope (SEM) images of hyphae before and after applying pulse voltage of fields of $10 \mathrm{kV}$ between wire electrodes with a gap length of $9 \mathrm{~cm}$ (Takaki 2021). It was confirmed that some hyphae are broken by applying pulse electric fields shown by arrows on Fig. (e). This result indicates that the pulse electric field stimulation is classified as physical facto with similar manner as scratching mycelia on the surface of the sawdust media for mushroom production (Kinkaki). Furthermore, new hyphae are developed and synthesis of crump connections are accelerated after applying pulse electric field stimulation (Kudo et al. 1999; Kaneko et al. 1987). Hydrophobin, which is involved in hyphal structure and architecture in fungi, would be involved in new hyphae generation after pulse stimulation. Expression of Hydrophobin in vegetative hyphae was confirmed to increase with rate of $230 \%$ at one day after the stimulation (Takaki et al. 2014). Some enzymes, including laccase and protease, were also confirmed to be activated by pulse electric field stimulation (2018; Kaneko et al. 1987; Ohga and Iida 2001). 

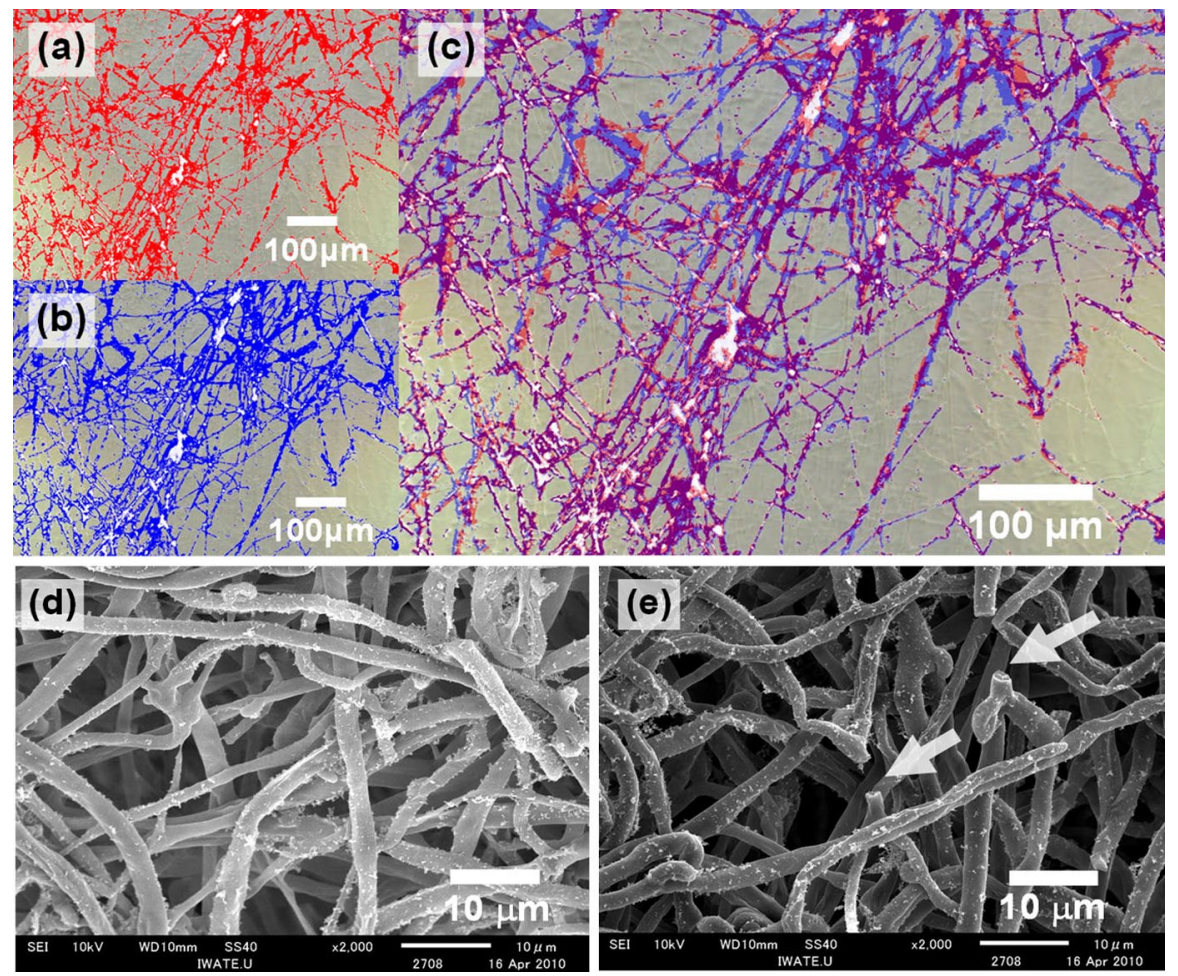

Fig. 73 Microscopic images of $L$. edodes hyphae a before and $\mathbf{b}$ after applying $5 \mathrm{kV} / \mathrm{cm}$ pulse electric field with pulse width of $100 \mathrm{~ns}$ and 500 times repetition. c Superimposed image of two images (a) and (b). d and e SEM images of $L$. edodes hyphae before (d) and after (e) applying $10 \mathrm{kV}$ pulse voltages. White bar indicates scale of $100 \mu \mathrm{m}$ in $\mathbf{a}, \mathbf{b}, \mathbf{c}$ and $10 \mu \mathrm{m}$ in $\mathbf{d}$ and $\mathbf{e}$

\section{Maintaining agricultural product freshness using high voltage and plasma}

Keeping freshness of agricultural products such as fruits and vegetables in food supply chain is a very important topic in the aspects of building a sustainable society in the world. The main factors of quality deterioration are respiration and spoilage driven by ethylene $\left(\mathrm{C}_{2} \mathrm{H}_{4}\right)$ and microbial contamination. Pulsed power technologies including time-modulated plasma have been used for various agriculture and food processing applications such as improvement of plant growth and electrostatic spray of agricultural chemical. The radicals produced by atmospheric non-thermal plasma, which has a high chemical oxidation potential, can contribute to rapid decomposition of $\mathrm{C}_{2} \mathrm{H}_{4}$. and inactivation of microorganisms. The electric field generated by high-voltage power supply can contributes to collecting airborne bacteria, which can also improve the environment of storage fruits and vegetables. The atmospheric non-thermal plasma can be generated with a compact high-voltage power supply and electrode system and generation of it does not require a special environment, 
which realizes low cost and simple process with compact device. In this section, degradation of $\mathrm{C}_{2} \mathrm{H}_{4}$ and microorganisms using time-modulated non-thermal plasma and its practical applications are described.

\subsection{Conventional technology to keep quality of agricultural product}

Main factors of quality deterioration are respiration and spoilage driven by ethylene $\left(\mathrm{C}_{2} \mathrm{H}_{4}\right)$ and microbial contamination. Since these effects can be suppressed in low temperature, a cold chain, a temperature-controlled supply chain, has been expanded, especially in developed countries (Onwude et al. 2020). The cold chain for agricultural products generally starts from pre-cooling, followed by cold storage, refrigerated transportation, cold auction room at a wholesale market, cold showcase at a retailing store, and a domestic refrigerator. The pre-cooling is used to reduce temperature of agricultural produce from temperature in the field to that of the storage just after the harvest for preserving quality. Several methods are used as precooling such as air cooling, hydrocooling, icing, and vacuum cooling. It is noted in a cold chain that the optimum temperature depends on the type of fruits and vegetables, and too low a temperature causes chilling injury.

Controlled atmosphere (CA) storage and modified atmosphere packing (MAP) are methods to reduce ripening and aging of products by controlling gas composition surrounding fruits and vegetables, which contributes to suppression of $\mathrm{C}_{2} \mathrm{H}_{4}$ and microbial spoilage. In general, the rate of respiration of agricultural products drastically decreases in the atmosphere of $2-10 \%$ of oxygen and $0.5-10 \%$ of carbon dioxide level. CA storage is a technology which stores the products in the optimum gas condition such as poor oxygen and rich nitrogen. The poor oxygen condition is usually obtained by combustion, adsorbing oxygen with molecular sieve or separating nitrogen with hollow fiber membrane. The carbon dioxide gas injection to CA storage is produced by the propane combustion. Similar concept is used in MPA, in which the optimum condition of gas mixture is used as atmosphere inside polymeric film package. Recently, 1-methylcyclopropene (1-MCP), an effective and efficient ethylene inhibitor, has been used for suppression of $\mathrm{C}_{2} \mathrm{H}_{4}$ spoilage.

The microbial spoilage is caused by bacteria and fungi such as mold and yeast. The microorganism contamination in pre-harvest period causes cultivation environments such as soil, irrigation water and manure. In post-harvest period, some microorganisms can move to other products from the contaminated stuffs via surface contact during storage and distribution. Furthermore, airborne microorganisms such as aerosolized bacteria can adhere on productions. There microorganisms cause not only quality losses but also health hazards by endotoxin and allergy. The damage of products by microorganisms does not appear immediately, but appears over time during storage and distribution in post-harvest period. Therefore, treatment of microorganisms after harvesting and during storage and distribution are very important. To control microorganism on the products, ozone and chlorine dioxide have been used as alternative methods to chemical treatments used in the past, such as ethylene oxide, formaldehyde, propylene oxide and methyl bromide. Ultraviolet ray has been reported as an effective method to inactivate microorganism by inhibiting replication 
of DNA which has specific absorption at wavelength of $254 \mathrm{~nm}$ (Hamanaka et al. 2011).

\subsection{Keeping freshness through inactivation and removal of airborne bacterial}

In the food industry, foods are contaminated by adhering microorganisms composed of bacteria and fungi via surface contact and via the air, which are composed of bacteria and fungi. The contamination of vegetables surface with bacteria cannot be excluded, because anything that comes into contact with vegetables has the potential to cause contamination. Possible sources of contamination are soil, water, handling of the products, harvesting and processing equipment and airborne bacteria. For example, in a rice storage facility, the concentration of microorganisms such as aerobic bacteria, molds and yeasts is $500-2000 \mathrm{CFU} / \mathrm{m}^{3}$ and increases $10-100$ times in a harvest season, which is a food safety issue. Thus, it is necessary to reduce the contamination by the inactivation of bacteria to avoid decay. The numbers of viable bacteria and coliform bacteria groups are closely related to potential danger of food poisoning and epidemic diseases. Thus, they are generally treated as the indicator of hygienic quality and environmental pollution.

Generally, technologies such as air filtration, UV irradiation, chemical aerosolization and ozone treatment have been practically used to control microorganisms, while they have limitations on the waste disposal treatment and the inactivation rate and speed. Plasma treatment system using high-voltage power supply can contribute to the quick and efficient control of air bone microorganism contaminations with two effects; microorganisms removal by the electrostatic precipitation effect (Koide et al. 2013) and inactivation by chemical active species reactions (Kolb et al. 2012; Ito et al. 2012; Ehlbeck et al. 2011).In this section, the mechanisms of prohibition of airborne bacterial infection, and the two effects of the plasma treatment are described.

\subsubsection{Collecting airborne bacteria using electrical precipitation}

Electrical precipitation is a micro-scale particle collection method by charging the particles and using an electrostatic force with a high collection efficiency. The electrical precipitation techniques have been widely used not only in the industry for environmental protection (Mizuno 2000; Huang et al. 2015), but also used for cleaning indoor air to collect fungi and pollen dusts as the healthcare applications. Electrical precipitator (ESP) is particle collection system using electrostatic force, and consists of high-voltage wire electrodes and grounded plane electrodes, in which the electric field appears between the electrodes. The particles are injected between the electrodes, where the particles are charged up by diffused ions induced from highvoltage wire electrode (Sudrajad and Fitri 2015). Following the charging the particles up, the charged particles are accelerated with electrical field between the electrodes, resulting particles are accumulated on the plane electrode surface, as shown in Fig. 74. 


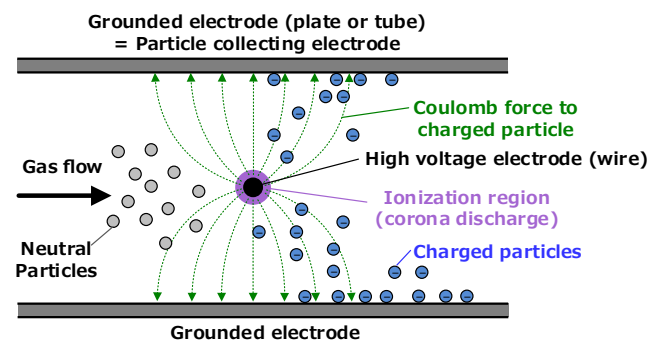

(a)

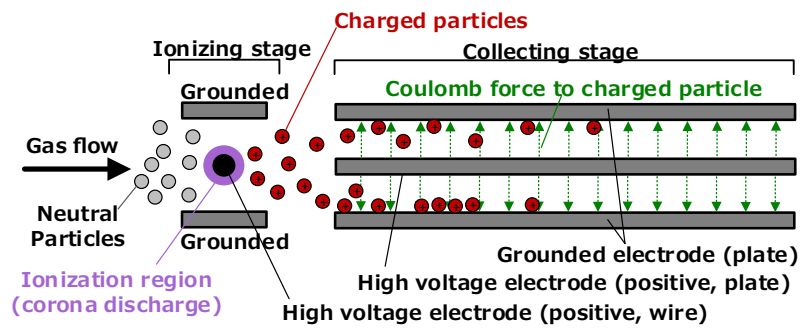

(b)

Fig. 74 Schematics of $\mathbf{a}$ one stage and $\mathbf{b}$ two stages type ESPs

The particle collection efficiency can be estimated by the following equation,

$$
\eta(\%)=1-\exp (-\omega A / Q)^{k}
$$

where $\omega$ means the effective migration velocity of particles, $A$ the total surface area of collecting electrodes, $Q$ the gas flow rate, and $k$ a constant value depends on types of particle and ranges from 0.4 to 0.6. This equation is well-known Deutsch-Anderson equation modified by Matt and Öhnfeldt. $\omega$ can be estimated by following equations

$$
\begin{gathered}
\omega=(q E / 6 \pi \mu a) C_{\mathrm{m}}, \\
C_{\mathrm{m}}=1+\alpha \lambda / a
\end{gathered}
$$

where $q$ means the particle charge, $E$ the electric field, $\mu$ the gas velocity, $a$ is particle radius, $C_{\mathrm{m}}$ the Cunningham correction to Stokes's law, $\lambda$ the mean free path of the gas molecules, and $\alpha$ the dimensionless parameter. In room temperature and pressure, $\lambda$ and $\alpha$ are roughly estimated as $0.069 \mu \mathrm{m}$ and 0.86 , respectively (Takahashi 2021). The diameter of airborne microorganism particles is in the range from 0.5 to $20 \mu \mathrm{m}$. ESP can collect the particles of micro-size with high efficiency. Therefore, ESP is suitable for collecting airborne bacteria. About applied voltage polarity, the negative voltage is commonly used than the positive, because most of the microorganisms are negatively charged. Moreover, the corona onset voltage of negative is lower than that of positive (Mainelis et al. 2002a). 
As an example of the use of the ESP, Koide et al. have developed an ESP device for collecting ground rice husks contaminated by microorganisms such as bacteria, molds and yeasts (Koide et al. 2013). In the system, ESP is consisted of wire-tocylinder electrode with a 36 inner diameter and $300 \mathrm{~mm}$ length. A DC high voltage of $-6 \mathrm{kV}$ is applied to the wire electrode. The corona current is approximately several $\mu \mathrm{A}$, and the consumed energy is less than $1 \mathrm{~mW}$. The average size of the particle is $130 \mu \mathrm{m}$, and the particle collection efficiency reaches $90 \%$. The microbial removal efficiency which includes microbial collection is approximately $99.95 \%$ is higher than the collection efficiency, as shown in Fig. 75. Han et al. reported that P. fluorescens and B. subtilis with a diameter of $0.8 \mu \mathrm{m}$ were removed with $72 \%$ efficiency using a cylindrical tube type ESP with an applied voltage of $-7 \mathrm{kV}$ (Han et al. 2010). ESP system was developed by Mitchell et al. for hatching cabinets. The developed ESP system reduced 85-93\% aerobic bacteria and enterobacteriaceae. ESP can contribute for reducing the infection risk through collecting airborne and dust particles, because salmonella is transmitted to eggs by airborne with dust. The developed ESP system also reduced the number of salmonella of cecal contents in a poultry house with reducing rate of $3.4 \mathrm{log} \mathrm{CFU} \mathrm{g}^{-1}$ (Mitchell et al. 2002). ESP was used to reduce the infection risk by viruses (Alonso et al. 2016). Aerosols in hog raising farm sometimes contains viruses such as influenza A, porcine reproductive and respiratory syndrome, porcine epidemic diarrhea, and S. aureus, which work as swine pathogens. The experimental results showed that the concentration of aerosols with particle size from 0.4 to $10 \mathrm{~mm}$ was reduced with $0.5-1.9 \operatorname{logs}$ by the ESP system. Airborne microorganisms of B. subtilis var. niger (BG) spores and vegetative cells were collected by newly developed ESP worked as bioaerosol sampler (Nonomura et al. 2009; Mainelis et al. 2002b). The collection efficiency for the bioaerosols are $90-100 \%$ at $4 \mathrm{kV}$ charging and precipitating voltages.

Fig. 75 Concentrations of microorganisms (bacteria, molds and yeasts) at different ESP voltages. ND indicates that mold and yeast concentration was undetectable levels $(n=6)$. Reprinted from Koide et al. 2013

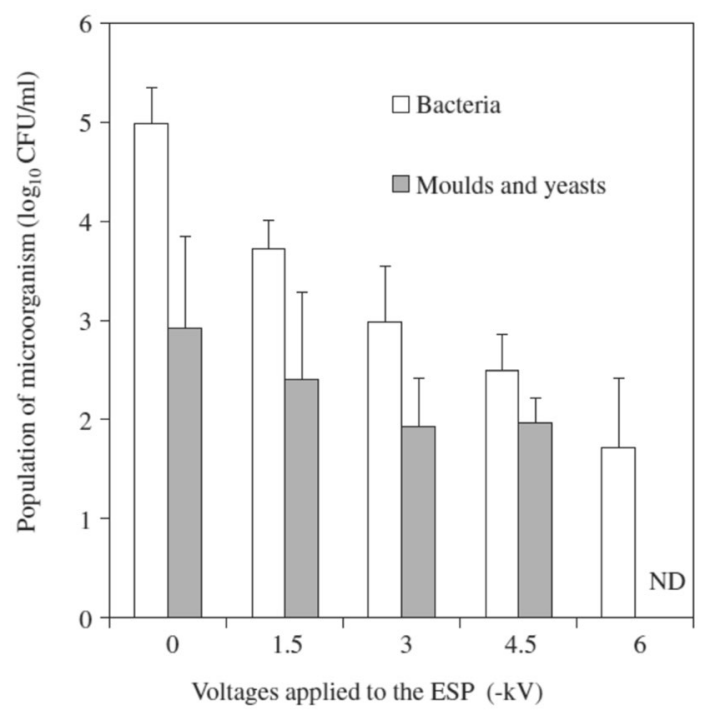

Springer 
Maintaining quality of agricultural products was improved using electric field irradiation through reducing activity of the products. Wang et al. reported effects of electric field pre-treatment during storage on maintaining tomato fruit quality and extending the shelf life. Green mature tomatoes were exposed to a positive or negative electric field of various strengths for $2 \mathrm{~h}$. The results indicated that the electric field of $-2 \mathrm{kV} / \mathrm{cm}$ in strength was effective for maintaining tomato quality, as shown in Fig. 76 (Wang et al. 2008). Peaks of respiration and ethylene production of tomato fruit during storage were shifted to longer days by pre-treatment with $-2 \mathrm{kV} / \mathrm{cm}$ strength electric field. The shifted days for of respiration and ethylene production peaks were 6 and 3 days, respectively. It was also confirmed that malondialdehyde content and electrical conductivity in tomato fruit were suppressed by exposing negative electric field during storage. The shelf life of strawberries (Fragaria $\times$ ananassa) was also extended using high-electric field treatment (Kharel and Hashinaga 1996). The treatment with $4.3 \mathrm{kV} / \mathrm{cm}$ in strength with duration of
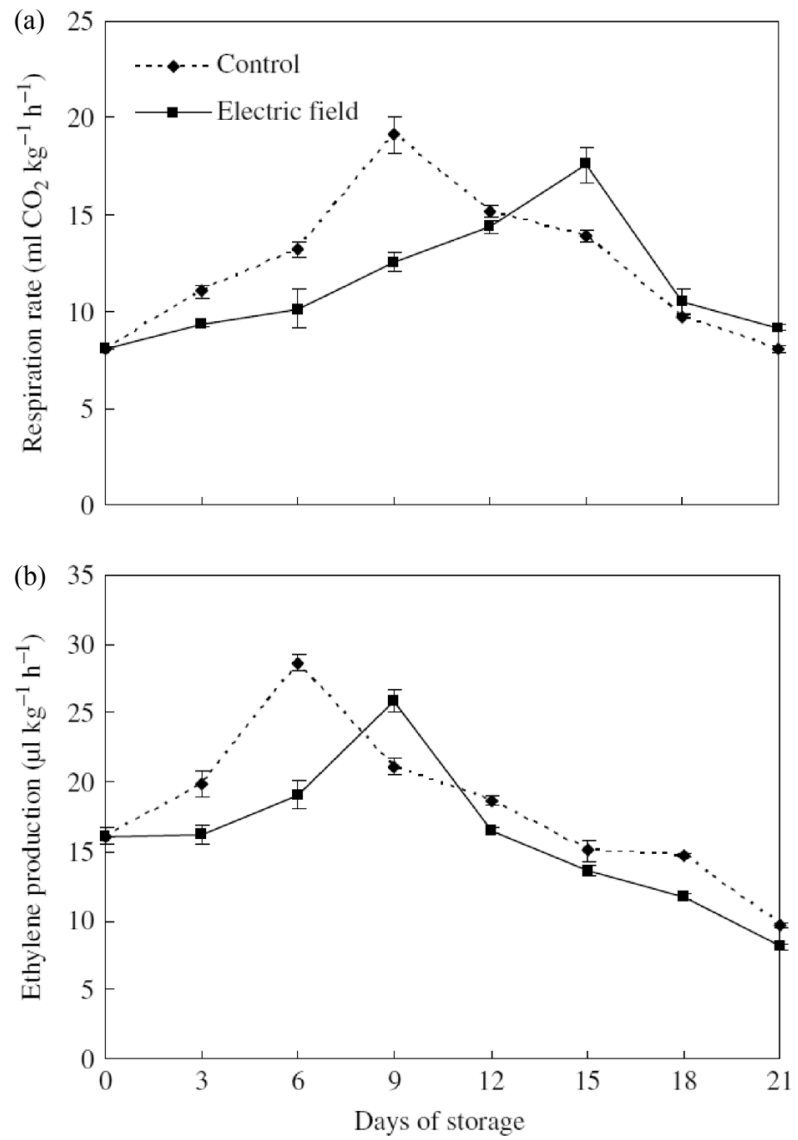

Fig. 76 Effects of $-2 \mathrm{kV} / \mathrm{cm}$ electric field treatment on respiration rate (A) and ethylene production (B) of tomato fruit during storage. (Wang et al. 2008) 
$0.5-1.5 \mathrm{~h}$ contributed to reduce the strawberry rotting. The results also showed that the continuous exposure by the electric field was effective for reducing the rotting.

\subsubsection{Inactivation of airborne bacteria using corona discharge}

There are many studies on sterilization of bacteria such as E. Coli, S. Aueus, B. Atrophoeus and Salmonella on agar plates (Sun et al. 2011; Fernández and Thompson 2012), and on the surface of agricultural products such as apples (Ito et al. 2021; Kilonzo-nthenge et al. 2018), by discharge plasma irradiation. The discharge plasmas produce chemically active species, ions and electron, UV radiation, intense high electric field and pressure waves (Sieck et al. 2000; Herron and Green 2001; Nakagawa et al. 2018; Lowke and Morrow 1995). The diffusion of chemically active species from plasma directly or plasma irradiated medium into biological cells. This diffused reactive species easily induce significant damage in the cell membrane and major cell constituent ( $\mathrm{Lu}$ et al. 2014).

Many kinds of non-thermal plasma sources have been used for inactivation of airborne bacteria. In particular, the dielectric barrier discharges (DBDs) have been frequently used for inactivation of bacteria with typical residence time of bacteria in plasma region of $10^{-3}$ to $10^{-4} \mathrm{~s}$. This short residence time is due to DBD reactor configuration of short gap (several $\mathrm{mm}$ ) with high air velocity (1-10 m/s) (Masotti et al. 2019). Gallagner et al. developed a small cylinder array type DBD reactor for inactivation of airborne bacteria as shown in Fig. 77 (Gallagher et al. 2007). The DBD is produced between the cylinder array electrodes. The airborne bacteria are flowed into the plasma region and is treated in short time duration.

Due to the strong oxidizing ability of ROS, various kinds of components of cell react with ROS, resulting some irreversible changes in the cell, leading to the destruction of dehydrogenase and breaking respiratory system down of the cell in microorganisms. The main reactions for inactivation of bacteria are the oxidation of SH-group, lipid, protein, DNA and RNA through reactions by ROS (Gaunt et al. 2006). Especially, the amino acid chains are easily oxidized through the reaction with ROS (Hyuk et al. 2006). If the unsaturated fatty acids in the cell membrane are oxidized with reaction by ROS, the cell membrane is ruptured through the chain

Fig. 77 A dielectric barrier discharge airborne bacteria inactivation system using cylinder type electrode array (Gallagher et al. 2007)

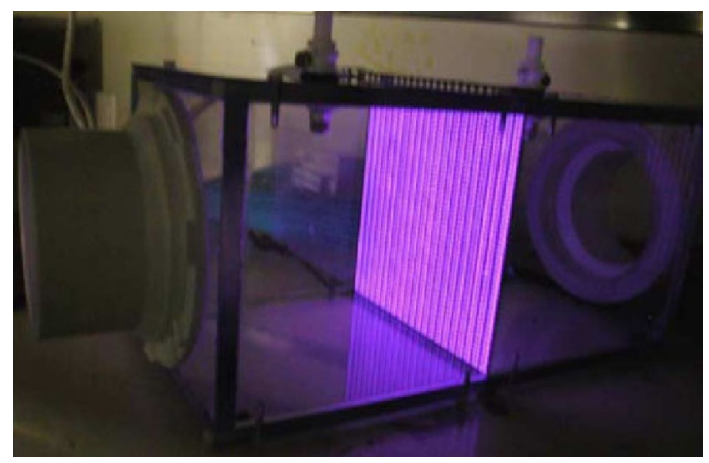


reaction of lipid peroxidation, as shown in Fig. 78 (Liang et al. 2012). In Fig. 78, it can be confirmed by a scanning electron microscope (SEM) images of B. subtilis that the membrane rupture is caused by the plasma treatment, resulting the intracellular component leakage (Gaunt et al. 2006). If the hydroxyl radical (OH) oxidizes the sugar moiety through the lipid peroxidation, DNA is easily damaged and is destructed (Mizuno 2000; Gaunt et al. 2006; Hirst et al. 2015). In general, the lifetime of hydroxyl radical is several microseconds, and its diffusion length is estimated as several tens $\mathrm{mm}$ in gas and $10^{-5}-10^{-6} \mathrm{~m}$ in aqueous phase (Takahashi et al. 2019a; Takeuchi and Yasuoka 2021). Therefore, hydroxyl radical is produced from hydrogen peroxide $\left(\mathrm{H}_{2} \mathrm{O}_{2}\right)$ and superoxide in liquid through Fenton oxidation reactions as mediation process (Han et al. 2014).

In the case of airborne bacteria, the inactivation by ozone takes $10^{2}-10^{3} \mathrm{~s}$ owing to the low reaction rate and the high concentration of ozone has to be retained, which has same disadvantage of the ozone treatment methods (Liang et al. 2012). The reaction rate of hydroxyl radical with bacteria is approximately $10^{3}-10^{4}$ higher than that of ozone and can be major player for inactivation in plasma with short residence time (Gallagher et al. 2007). Moreover, the synergistic effects of other ROS such as ozone and atomic oxygen are induced by hydroxyl radical, leading the enhancement of inactivation efficiency (Liang et al. 2012). The hydroxyl radical $(\mathrm{OH})$ and the superoxide dissolve into aerosol aqueous solution. They are converted

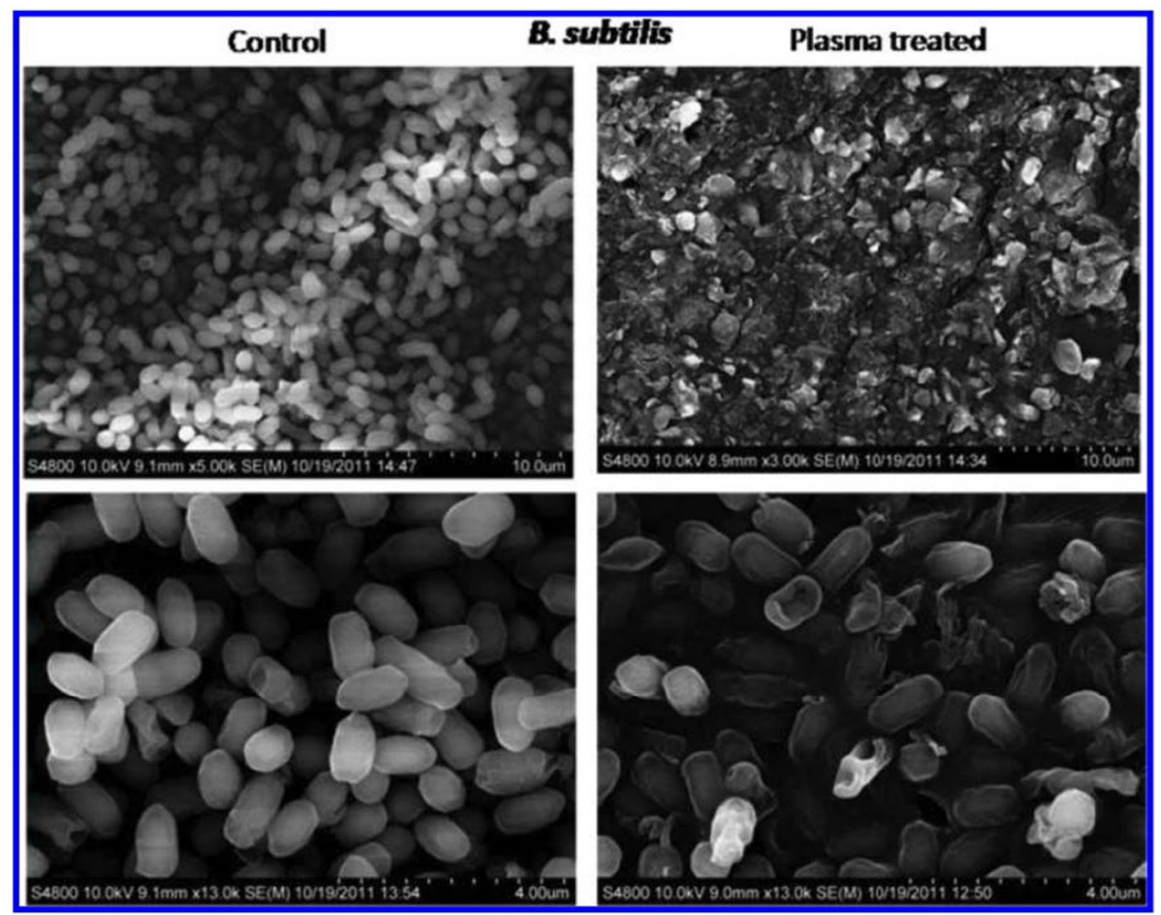

Fig. 78 SEM images of B. subtilis treated by the plasma (Liang et al. 2012) 
to stable hydrogen peroxide $\left(\mathrm{H}_{2} \mathrm{O}_{2}\right)$ in the aerosol aqueous solution, causing oxidative damage of airborne bacteria.

Two oxidation stresses by superoxide dismutase and catalase mainly contribute inactivation of bacteria (Vaze et al. 2017). The bacteria are electrically charged by ions and electrons in the plasma. The charged species such as ions and electrons play an important role in the inactivation through synergy effects of other chemically active species. The charged particles causes the poration of cell in several microseconds by locally enhanced electric field owing to the charge-up (Noyce and Hughes 2002). The etching of membrane is also caused by ion bombardments (Hyuk et al. 2006). These phenomena by charged particles enhance oxidation reaction of membrane with ROS. The synergy effect was also confirmed to enhance the membrane reaction with ozone. The act of ozone to the bacteria was enhanced by the synergy effect of charged particles (Vaze et al. 2010). Zhou et al. analyzed numerically the distributions of ions and bacteria in a duct using fluid model. In the calculation, the ion concentration and an air velocity in the duct were set to be order of $10^{12} \mathrm{~m}^{-3}$ and $2-5 \mathrm{~m} / \mathrm{s}$, respectively. From the numerical analysis and the experiment, it was confirmed that inactivation of $E$. coli had strong relation to the negative ions distribution (Zhou et al. 2016).

\subsection{Keeping products quality through decomposition of ethylene}

Ethylene $\left(\mathrm{C}_{2} \mathrm{H}_{4}\right)$ is a hormone in plants. It has an aging effect to fruits and vegetables, and excessive maturation due to prolonged exposure leads to deterioration in their quality (Abeles et al. 1992). Some fruits and vegetables, such as apples emit large amount of $\mathrm{C}_{2} \mathrm{H}_{4}$ into the air. The $\mathrm{C}_{2} \mathrm{H}_{4}$ emitted from the fruits and vegetables has also autotoxic effects (Silva et al. 1999). Thus, the $\mathrm{C} 2 \mathrm{H} 4$ concentration should be kept low, e.g., less than 1 ppm during the transport of ethylene-sensitive fruits loaded together in one container and the long storage. In this section, removal of ethylene using high-energy electron accelerated by pulse voltage and corona discharges for contribution of keeping quality of agricultural products are outlined.

Table 4 shows the comparison of the $\mathrm{C}_{2} \mathrm{H}_{4}$ removal methods. $\mathrm{C}_{2} \mathrm{H}_{4}$ in the container and storage is usually removed by conventional methods such as venting and absorption (Abeles et al. 1992). The venting is the easiest way to reduce $\mathrm{C}_{2} \mathrm{H}_{4}$ concentration. However, to keep freshness, the temperature inside the container and storage should be kept low and its fluctuation should be minimized. The exchange of large amount of gas leads to the rapid temperature change, which leads to the loss of freshness of fruits and vegetables. The absorption using absorbent materials can reduce $\mathrm{C}_{2} \mathrm{H}_{4}$ easily; however, its amount of absorption is limited by the size of the absorbent and their regeneration and disposal after use remain an issue. To overcome these limitations of conventional methods, various methods have been proposed. The ozonation is a method using the oxidation reaction of ozone $\left(\mathrm{O}_{3}\right)$ (Boonkorn et al. 2012). $\mathrm{O}_{3}$ is a powerful oxidant, and the $\mathrm{C}_{2} \mathrm{H}_{4}$ containing in the gas is decomposed into by-products, and finally $\mathrm{CO}_{2}$ and $\mathrm{H}_{2} \mathrm{O}$ through the oxidation reactions. Although it is effective in removing ethylene, ozone has a harmful effect on not only fruits and vegetables, as shown in Fig. 79, but also human body and 
Table 4 Comparison of the $\mathrm{C}_{2} \mathrm{H}_{4}$ removal methods

\begin{tabular}{llllll}
\hline & $\mathrm{C}_{2} \mathrm{H}_{4}$ Removalspeed & Safety & Size and weight & Performancestability & Operability \\
\hline Venting & Excellent & Excellent & Good & Excellent & Inferior $^{\mathrm{a}}$ \\
Absorption & Excellent & Excellent & Average & Inferior & Inferior \\
Photocatalyst & Inferior & Good & Inferior & Average & Excellent $^{\text {Ozone }}$ \\
Inferior & Inferior & Good & Excellent & Inferior $^{\text {b }}$ \\
Plasma & Excellent & Good & Good & Excellent & Excellent \\
\hline
\end{tabular}

${ }^{a}$ Venting leads temperature change, which causes negative effect for keeping freshness of fruits and vegetables

${ }^{\mathrm{b}}$ Ozone causeas injury on fruits and vegetables and damage to facility

Fig. 79 Apple injured by ozone

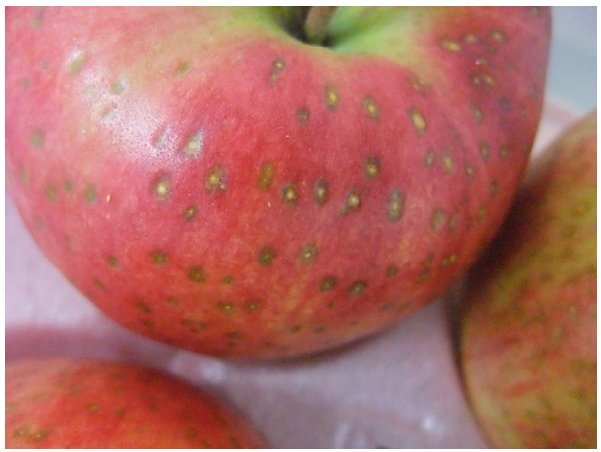

damages the structure of container and storage (Moretti et al. 2010; Skog and Chu 2001). Photocatalytic reaction is also effective in decomposition of $\mathrm{C}_{2} \mathrm{H}_{4}$. The photocatalytic reaction is induced on the surface of photocatalyst such as $\mathrm{TiO}_{2}$, which is irradiated by ultraviolet lights. For example, Maneerat et al. confirmed that $20 \mathrm{ppm}$ concentration of $\mathrm{C}_{2} \mathrm{H}_{4}$ was filled in storage box of $5 \mathrm{~L}$ capacity was completely decomposed using $\mathrm{TiO}_{2}$ solution which was activated by ultraviolet-A (UVA) irradiation with dose of $0.1 \mathrm{~mW} / \mathrm{cm}^{2}$ (Maneerat et al. 2003). This decomposition of $\mathrm{C}_{2} \mathrm{H}_{4}$ contributed for elongation of agri-food quality with delaying the ripening of green into red-colored tomatoes. In the photocatalytic reactions, $\mathrm{C}_{2} \mathrm{H}_{4}$ approached on the reaction zone on the surface can be rapidly decomposed. Because the reaction zone is limited on only the surface, this technology is much safer and less affected on fruits and vegetables (Yamazaki et al. 1999; Chavadej et al. 2007). Since its reaction rate is not high, the large surface area is required for sufficient treatment, which makes the system large and heavy.

Discharges produce instantly non-thermal plasma. In the plasma, various powerful oxidants are produced with high density, and ethylene can be decomposed with very high reaction rate. For instance, the decomposition speed of 200 ppm ethylene by a plasma discharge system is on order of $10^{-7} \mathrm{~mol} / \mathrm{W}$ s (Takaki et al. 2015), which is approximately three order higher than $3 \times 10^{-10} \mathrm{~mol} / \mathrm{W} \mathrm{s}$ of decomposition efficiency of photocatalytic reactions (Yamazaki et al. 1999). Additionally, the 
plasma is only produced by electricity, and its energy density in the plasma is very high, which has a high potential to make the system compact and light.

Intense pulsed electric fields were used for prolonging the quality of agri-foods through the $\mathrm{C}_{2} \mathrm{H}_{4}$ decomposition (Liu et al. 2011). The intense pulsed electric field was produced by applying pulsed voltage with $2 \mathrm{kV}$ strength and $1.0 \mu$ s width to wire electrodes located $1 \mathrm{~mm}$ apart from ground mesh electrodes. The intense electric fields produced 20-30 $\mu \mathrm{A}$ discharge currents which containing energetic electrons. The results showed that the $\mathrm{C}_{2} \mathrm{H}_{4}$ concentrations in the storehouse was reduced from 120 to $40 \mathrm{ppm}$ by applying intense pulsed electric fields in the storehouse. The ripening of bananas with preservation period of 21 days was also delayed. They reported that the direct dissociation of $\mathrm{C}_{2} \mathrm{H}_{4}$ by energetic electron $\left(>3.5 \mathrm{eV}\right.$ ) impact was main pathway of the $\mathrm{C}_{2} \mathrm{H}_{4}$ decomposition. It was noted that the required energy of $3.5 \mathrm{eV}$ for dissociation of $\mathrm{C}-\mathrm{H}$ bond was lower than that of $\mathrm{O}-\mathrm{H}$ bond $(4.5 \mathrm{eV})$. Therefore, the energetic electrons selectively contributed for dissociation of $\mathrm{C}-\mathrm{H}$ bond even a humid air condition (Liu et al. 2011).

Different types of discharges have been used to generate non-thermal plasma. DC or AC corona type discharge generates small localized non-thermal plasma at needle tips and vicinity of wire electrodes. Because corona electrodes can be easily and simply consisted with needles and conventional DC or AC high voltage power supply can be adapted, the corona discharge system can be easy to install and robust against contaminations. On the other hands, the volume of plasma is localized and reaction area for ethylene $\left(\mathrm{C}_{2} \mathrm{H}_{4}\right)$ decomposition is limited, the miniaturization of the system is difficult. Dielectric barrier discharge (DBD) is one of methods to produce the non-thermal plasma at atmospheric pressure. The DBD is usually generated between two electrodes, of which at least one is covered by a dielectric barrier such as ceramics, with a voltage of AC or pulse applied to these electrodes. The DBD is characterized by a high electron temperature of $10 \mathrm{eV}$ and a high electron density of $10^{20} \mathrm{~m}^{-3}$ in comparison to DC corona discharge (Wang et al. 2012; Eliasson and Kogelschatz 1991a). Therefore, the $\mathrm{C}_{2} \mathrm{H}_{4}$ decomposition efficiency of the DBD is approximately two-order higher than that of the DC corona discharge (Takaki et al. 2020). The nanosecond pulse voltages with high voltage rise speed can make the discharges uniform. Additionally, the reduced electric field increases with increasing the discharge time lag by increasing voltage rise speed, which enhances the radical production by electron impacts such as:

$$
\mathrm{e}+\mathrm{O}_{2} \rightarrow 2 \mathrm{O}
$$

Since the oxidation potential of $\mathrm{O}$ is very high, $\mathrm{C}_{2} \mathrm{H}_{4}$ can be quickly oxidized as following reactions (Takaki et al. 2020).

$$
\begin{gathered}
\mathrm{O}+\mathrm{C}_{2} \mathrm{H}_{4} \rightarrow \mathrm{CHO}+\mathrm{CH}_{3}, \\
\mathrm{O}+\mathrm{C}_{2} \mathrm{H}_{4} \rightarrow \mathrm{CH}_{2} \mathrm{CHO}+\mathrm{H} .
\end{gathered}
$$

The rate constants of reactions (43) and (44) are $3.45 \times 10^{-18} \times T^{2.08}\left[\mathrm{~cm}^{3} / \mathrm{s}\right]$ and $2.0 \times 10^{-18} \times \mathrm{T}^{2.08}\left[\mathrm{~cm}^{3} / \mathrm{s}\right]$, respectively, where $T$ is a temperature in $[\mathrm{K}]$. 


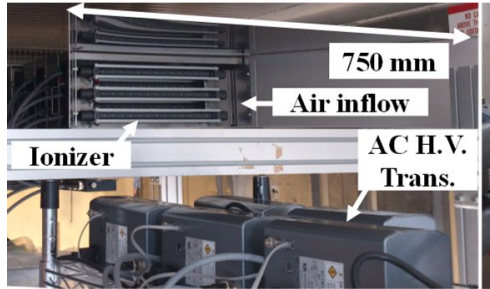

(a)

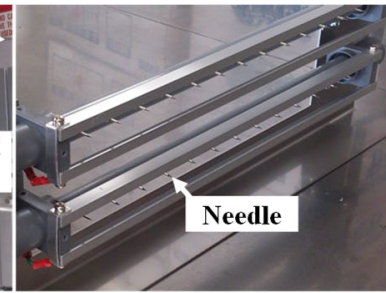

(b)

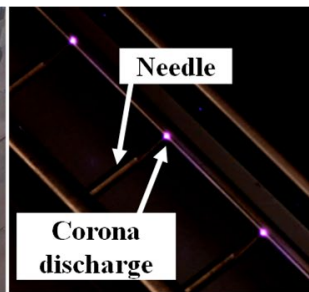

(c)

Fig. 80 Photographs of corona discharge type $\mathrm{C}_{2} \mathrm{H}_{4}$ removal devices a installed in a container, b ionizer unit in the device and corona discharges

Figures 80 and 81 show the ethylene removal devices utilizing corona discharge and DBD, respectively, for a $20 \mathrm{ft}$ refer container. The devices consisted of fans to vacuum air inside container and storage, discharge electrodes and catalysts. The gas treated by discharges passes through the catalysts to eliminate by-products of plasma treatment such as $\mathrm{O}_{3}$ and $\mathrm{CO}_{2}$ in the gas. The corona discharge type electrode is consisted of a bar type ionizer (Shishido electrostatic, BOS-400) driven by AC highvoltage transformer at commercial frequency (Shishido electrostatic, SAT-11) with an amplitude of $\pm 7.3 \mathrm{kV}$. The ionizer has 13 corona needles as shown in Fig. 80b and corona discharge occurs at the tip of each electrode as shown in Fig. 80c. The number of the ionizer installed in the device is 10 . The DBD type electrode is consisted of multi parallel rod electrodes covered by ceramic tube with a gap length of $1 \mathrm{~mm}$ as shown in Fig. 81b, and DBD occurs in the gap as shown in Fig. 81c (Takahashi et al. 2021; Takaki et al. 2020). AC voltage of $\pm 7.5 \mathrm{kV}$ with frequency of $500 \mathrm{~Hz}$ was generated by a compact winding transformer (Shishido electrostatic, Fig. 81d) driven by an inverter circuit consisted of MOFETs (Takahashi et al. 2019b) is applied to the electrodes. The $\mathrm{C}_{2} \mathrm{H}_{4}$ removal rate of both devices is approximately $60 \mathrm{mg} / \mathrm{h}$ with a $\mathrm{C}_{2} \mathrm{H}_{4}$ concentration of $100 \mathrm{ppm}$. The corona discharge type device has approximately a height of $200 \mathrm{~mm}$, a width of $750 \mathrm{~mm}$ and length of $1500 \mathrm{~mm}$ and 5 AC high-voltage transformers, each s a height of 220, a width of $90 \mathrm{~mm}$ and length of 110 in size, need to be installed externally. DBD type device has a height of $110 \mathrm{~mm}$, a width of $370 \mathrm{~mm}$ and length of $300 \mathrm{~mm}$, including internal compact high-voltage power supply system. These indicates that DBD type can be designed

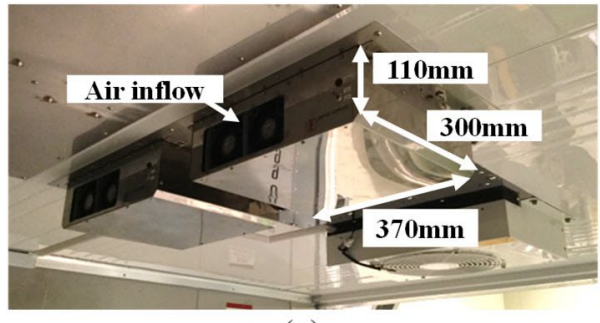

(a)

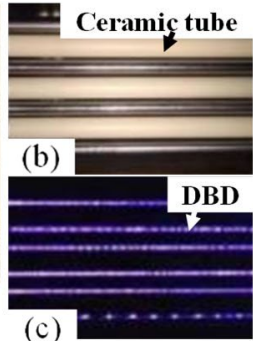

(c)

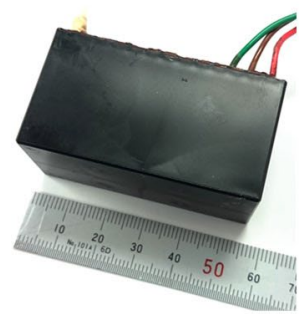

(d)

Fig. 81 Photographs of DBD type $\mathrm{C}_{2} \mathrm{H}_{4}$ removal devices a installed in a container, b electrode system, $\mathbf{c}$ $\mathrm{DBD}$ and $\mathbf{d}$ compact winding transformer 
to be compact. Figure 82 shows the $\mathrm{C}_{2} \mathrm{H}_{4}$ concentration in the $20 \mathrm{ft}$ container in which fruits and vegetables are mix loaded and DBD type $\mathrm{C}_{2} \mathrm{H}_{4}$ removal system is installed. The $\mathrm{C}_{2} \mathrm{H}_{4}$ concentration can be kept at low less than $1 \mathrm{ppm}$ with the system operation.

\section{Pulsed power applications for food processing}

Pulsed power can generate short-duration intense pulsed electric fields, nanosecond pulsed discharges, and intense shock waves via pulsed discharge. These phenomena have been used in post-harvest phase including food processing (Takaki et al. 2019; Ohshima et al. 2021). Especially, intense pulsed electric fields (PEF), in range from several nanoseconds to several microseconds of pulse width and from 0.1 to $80 \mathrm{kV} /$ $\mathrm{cm}$ of strength, have actively used as non-thermal treatment in food processing (Fincan and Dejmek 2002; Koubaa et al. 2015; Vorobiev and Lebovka 2008). For example, PEF pasteurization is technology of inactivation of pathogenic bacteria in liquid food and has potential as an alternative to thermal pasteurization processing. The advantage of PEF pasteurization compared with conventional thermal processing is that the attributes of liquid foods such as sensory, nutrition and health-promoting agents, are modified minimally or remained (Sánchez-Vega et al. 2014). The PEF pasteurization is induced through electrically poration in the biological membrane, i.e., electroporation (Barba et al. 2014; Deng et al. 2014). In addition, PEF processing is also used to extract health-promoting agents from vegetable or fruits, to improve drying process of foods, and to reduce enzyme activity in food processes. This section reviews the potential applications of PEF in food processing.

\subsection{Electroporation for food processing}

Almost applications of an intense pulsed electric fields (PEFs) in food processing are generally based on electroporation, in which biological membranes are pored through electrical breakdown of the membrane (Syed et al. 2017; Mohamed and Eissa 2012). The phenomenon of electroporation was investigated systematically
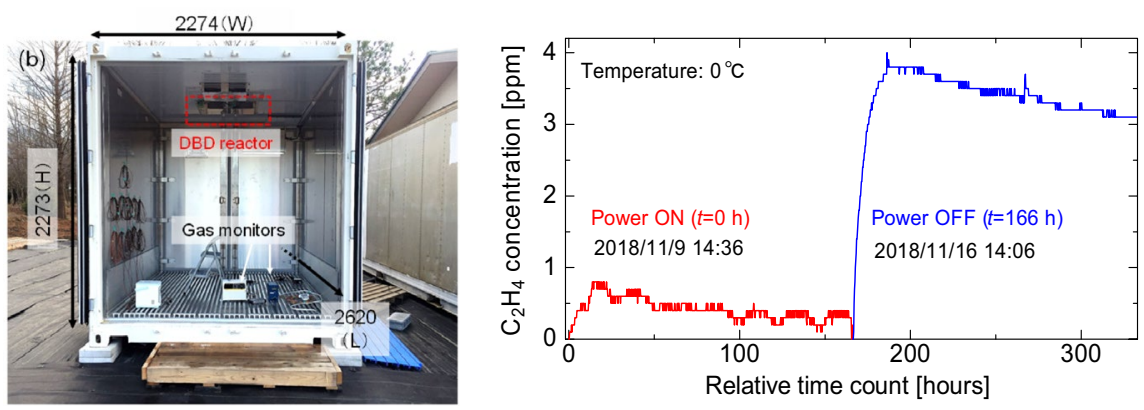

Fig. 82 Photograph of DBD $\mathrm{C}_{2} \mathrm{H}_{4}$ removal device installed container (a), and $\mathrm{C}_{2} \mathrm{H}_{4}$ concentration in container with operation of DBD $\mathrm{C}_{2} \mathrm{H}_{4}$ removal device during transportation 
by Zimmermann et al., and was explained as disruption of membrane by electromechanical compression (Zimmermann et al. 1974, 1976). Namely, the membrane works as capacitive component. The electrical charges on the membrane makes electromechanical force between the membrane by Coulomb force by applying electrical field. This mechanical stress induces the thinning of membrane in the case of compressible membrane. This phenomenon was confirmed by experiment (Zimmermann et al. 1974; Zimmermann 1982). The thickness of the membrane is determined by equilibrium between elastic restoring forces and Maxwell tension (i.e., compressive force) caused by charging up the membrane. Therefore, the equivalent thickness of membrane decreases with increasing electrical charge of membrane by increasing strength of the applied PEF. When the membrane thickness decreases to the critical value, the membrane becomes unstable resulting disruption of hydrophobic bonding the lipid bilayer, i.e., formation of pores in membrane. The pores are filled with conductive solutions of inside or outside of the membrane. As the result, electrical permeability of the membrane increases drastically, e.g., increase of eight orders in pure lipid bilayer. This rapid increase of the electrical conductivity causes a rapid discharge of accumulated electrical charges between the membrane. The typical values of PEF strength are in range from 1 to $20 \mathrm{kV}$. This critical PEF strength depends on size of cell, and is determined by membrane breakdown voltage of approximately $1 \mathrm{~V}$ (Akiyama and Heller 2017; Zimmermann 1982).

Phenomena of the electroporation are commonly classified as two groups: reversible and irreversible disruptions. In general, the pore size varies with PEF parameters such as strength, irradiation period and input energy. If the pore size is sufficiently small to allow recovery of membrane, the diffused lipid bilayers are rearranged, resulting to close the pores. This process is classified as reversible disruption. On the other hand, if the pore size is larger than the critical value at higher field strengths, the diffused lipid bilayers are no longer able to repair these perturbations (irreversible disruption). The reversible disruption of membrane can be used as electroporation (Kotnik et al. 2015; Jiang et al. 2014b) or electrofusion (Aroush et al. 2015; Asami 2016). The irreversible disruption can be used in inactivation of microorganism (Cregenzan-Alberti et al. 2015; Siemer et al. 2014; Pataro et al. 2013) or permeabilization of foods (Nakagawa et al. 2013).

\subsection{PEF pasteurization}

An intense PEFs have been used for inactivation of pathogenic bacteria in pre-harvest phase, airborne bacteria in post-harvest phase of agriculture as shown in chapters 5 and 6 . The PEF treatments have been also used for inactivation of microorganisms in liquid food, i.e., pasteurization in food processing in same manner of inactivation of bacteria in the agricultural field.

The PEF treatments for destruction microorganism as pasteurization have been investigated in scientific and practical from early 1990s (Barbosa-Canovas and Altunakar 2006). PEF pasteurization has advantages compared with conventional thermal pasteurization such as fresh-like products and high nutritional quality. Influences of PEF treatment on bioavailability of bioactive compounds contained in 
liquid foods were evaluated by some researchers and were summarized as review papers (Barba et al. 2015; Min et al. 2007; Castro et al. 2007). Especially, cow's milk is one of the most attractive liquid food for PEF treatments, because the milk contains protein with high concentration, in which the nutritional qualities are easily degraded by thermal treatment through the disnature of protein. Therefore, the effect of PEF pasteurization on quality of whole milk has been reported by some researchers (Zhao et al. 2013; Sharma et al. 2014a; Cregenzán-Alberti et al. 2014).

Yang et al. confirmed the effect of PEF treatments as non-thermal process on phenolic compound extraction from grape in wine processing, and on inactivation of spoilage microorganism in wine, beer and rice wine processing (Yang et al. 2016). Sharma et al. reported effect of PEF treatment with combination of pre-heating process on microbial inactivation in whole milk. The pre-heating was controlled as $55{ }^{\circ} \mathrm{C}$ in temperature with treatment period of $24 \mathrm{~s}$, following stepwise cooling. The PEF treatment was set to be $22-28 \mathrm{kV} / \mathrm{cm}$ in strength with a $20 \mu$ s pulse width at $10-60 \mathrm{~Hz}$ of pulse repetition rate. Pseudomonas aeruginosa, Escherichia coli (E. coli), Staphylococcus aureus and Listeria innocua were used as specimen of microorganisms. The experimental result showed that 5-6 log reduction of all microorganism specimens to the levels below detection limits (Sharma et al. 2014a). Sharma et al. also reported the reductions of 2-3 log in whole milk by PEF treatments with condition of $20.7-26.2 \mathrm{kV} / \mathrm{cm}, 20 \mu \mathrm{s}$ at $10-60 \mathrm{~Hz}$. This microbial reduction levels of PEF treatment are almost equal to those of thermal pasteurization with $63{ }^{\circ} \mathrm{C}$ for $30 \mathrm{~min}$ (low-temperature pasteurization) or $73{ }^{\circ} \mathrm{C}$ for $15 \mathrm{~s}$ (Sharma et al. 2014b). Typical PEF pasteurization system with pre-heating unit for whole milk is shown in Fig. 83 (Ohshima et al. 2016). Ohshima et al. investigated using the PEF pasteurization system with $40 \mathrm{kV}$ of voltage strength at $50 \mathrm{~Hz}$ of pulse prepetition rate. The whole milk including $E$. coli was used as specimen. The results showed that $E$. coli cells were not detected from treated milk. They confirmed that the processes of preheating and post-holding were effective for improving an efficiency of the pasteurization (Ohshima et al. 2016).

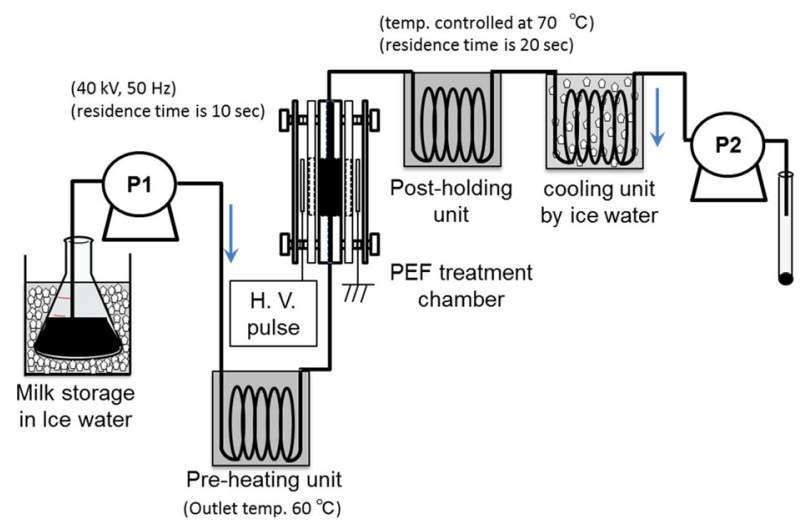

Fig. 83 Schematic of PEF pasteurization apparatus with pre-heating and post-holding units. Reprinted from (Ohshima et al. 2016) 
There are many research papers about effectiveness of PEF treatment on pasteurization of liquid food. However, thermal process is still mainly used in food industry. For industrial applications of PEF pasteurization, the development and optimization of PEF pasteurization system including power source, electrode configuration, preheat treatment, cooling unite, etc. For example, parallel plane electrode has been commonly employed in PEF pasteurization because of homogeneous electric field strength between the plane electrodes, i.e., homogeneous effect on pasteurization. On the other hand, the parallel plane electrode configuration has some disadvantages such as accompanying large joule heating loss and pressure drop of liquid food flow in processing. Ohshima and Sato evaluated the energy efficiency in in PEF pasteurization for various configurations of electrodes. They employed configurations of parallel plane, needle-to-plane, ring-to-cylinder, and spiral winding. The evaluation result showed that the PEF pasteurization efficiency depended strongly on the electrode configuration. The concentrated region in non-uniform electric field was effective for inactivation of microorganism (Ohshima et al. 1997; Sato et al. 2001). A novel textile electrode was also tried to use as PEF processing by Kitajima et al. The textile electrode was combined of polyester fiber with tungsten wires at $0.2 \mathrm{~mm}$ in diameter. The effectiveness of the textile electrode was confirmed in PEF processing for inactivation of E. coli. The inactivation efficiency had maximum at $7 \mathrm{kV}$ strength of applied voltage, and showed high value in low solution conductivity (Kitajima et al. 2007).

\subsection{PEF extraction of intracellular contents}

PEF extraction of intracellular contents is based on phenomenon of poration or disruption of biological membrane through electromechanical compression process by applying intense PEF (Zimmermann et al. 1975). PEF extraction including both reversible and irreversible disruptions of membrane, in which it is necessary to control the input energy into the cell membrane. The input energy from PEF to the membrane is generally controlled by electrical parameters such as PEF intensity, repetition rate and pulse width. This technology can be used in extraction of healthpromoting agents from vegetables and fruits, but also used as pre-treatment in food drying processes.

Some researchers have been confirmed that some intracellular contents, such as proteins including enzymes and waters, are extracted to the supernatant of cell suspension by applying PEF to the specimens. Ohshima et al. confirmed the extraction of the intracellular protein from yeast cells (Ohshima et al. 1995). They also investigated the effect of cell membrane exposure by PEF on extraction of intracellular protein and the recovery of target proteins using recombinant E. coli, as shown in Fig. 84 (Ohshima et al. 2000). The experimental result showed that the extraction efficiency of the target proteins (evaluated by enzymatic specified activity) depended on electrical parameters of PEF. The extraction selectivity of PEF extraction was higher than that of supersonic treatment. Shiina et al. reported that the recovery of extracted enzyme activities from a recombinant $E$. coli was improved by applying intermittent PEF treatment. The intermittent PEF 

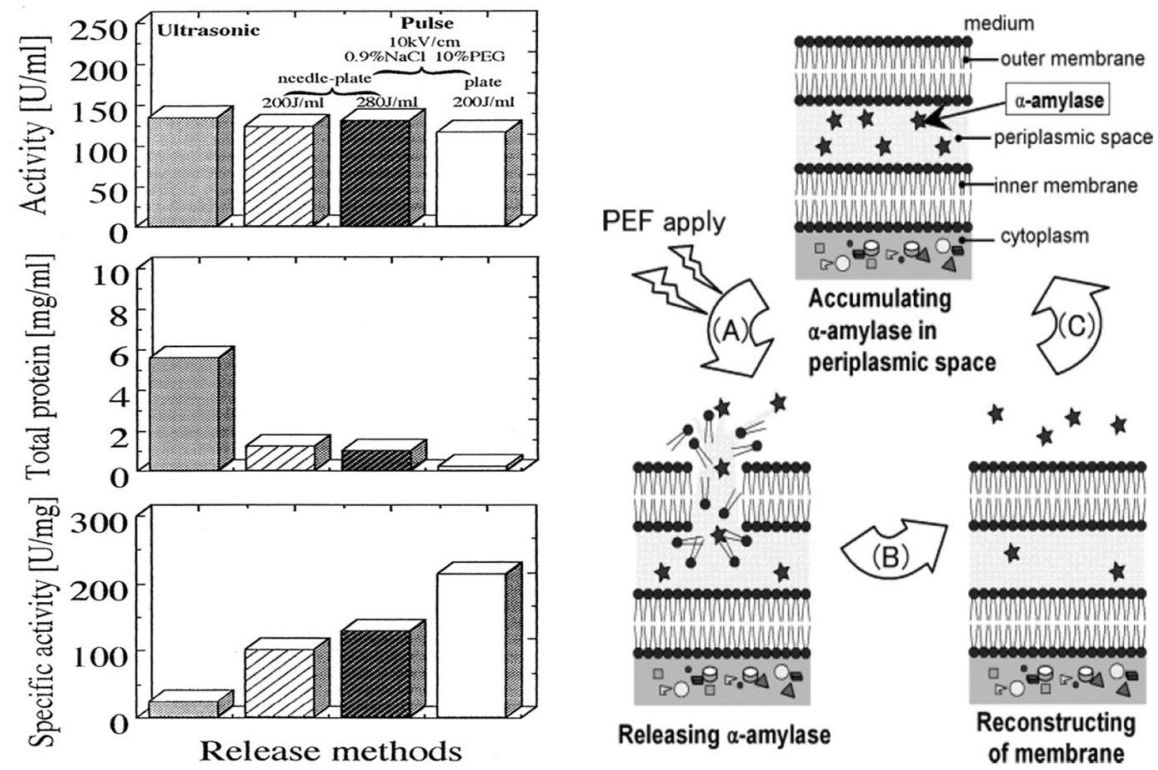

Fig. 84 Recombinant E. coli/pHI301A and $\alpha$-amylase activity recovery after PEF extraction of intracellular proteins. Reprinted from (Ohshima et al. 2000)

was effective for reversible disruption of cell membrane with high surviving rate of the cell (Shiina et al. 2004). They also reported effect of PEF treatment on production of $\alpha$-amylase produced by recombinant $E$. coli during cultivation. The extracted $\alpha$-amylase was approximately $30 \%$ of total $\alpha$-amylase production, which was defined as sum of extracellular and intracellular $\alpha$-amylase in the recombinant $E$. coli, by applying PEF of $12 \mathrm{kV} / \mathrm{cm}$ with intermittent as $50 \%$ duty $(30 \mathrm{~min}$ on and $30 \mathrm{~min}$ off). Nature $E$. coli has no function to release secrete protein from intracellular to extracellular. However, a PEF-assisted cultivation of E. coli enables the extracellular production of recombinant proteins (Shiina et al. 2007).

Mahnič-Kalamiza et al. discussed about possibility of PEF extraction of health-promoted compounds from residues of food processing such as seeds, peels, grape husks, and oil cakes. They also evaluated the effect of PEF treatment on extraction of variable compounds for biorefining agricultural and forestry residues such as stems, sawdust and leaves and bark, energy crops and municipal wastes, etc. (Mahnič-Kalamiza et al. 2014). This concept is almost same as modern green technology, which is important for sustainable food supply chain through renewable plant resources without agro-solvents (agro-chemical). Barba et al. also discussed the effectiveness of PEF treatment on extraction of valuable compound from by-products wastes in food processing. They used biomass from terrestrial plants, energy crops, crop residues, forestry residues, grape pomace, food wastes and beer waste brewing yeasts as specimens. These materials contains many bioactive compounds, especially polyphenols (such as phenolic acids, flavonol glycosides, anthocyanins, catechins), which have functions of antiviral, 
antibacterial, antifungal, anticancer and antioxidant effects. They also discussed about the usage of PEF treatment for biorefinery applications (Barba et al. 2015).

Recently, PEF treatments was applied for extraction of juices and nutritive molecules from agricultural products. Nakagawa et al. reported the effect of PEF treatment on polyphenol extraction from grape skins. The grape skins were immersed in distilled water between parallel plane electrode. Pulse-forming network (PFN) was used to control the pulse width and intensity of PEF. The efficiency of total polyphenol extraction was evaluated as gallic acid concentration in the solvent (water) by Folin-Ciocalteu analysis. The microscopic observation showed that membrane of anthocyanoplasts were raptured by PEF exposure, and the red-colored pigments in the anthocyanoplasts were extracted into the cell and solvent, as shown in Fig. 86 (Nakagawa et al. 2013). The polyphenol extraction was enhanced with increasing pulse width in the PEF treatment, i.e., the energy required for polyphenol extraction decreased with increasing pulse width, as shown in Fig. 85.

\subsection{PEF treatment for protein modifications}

In some food processes such as brewing and fermenting, inactivation of enzyme is final step to distribute for consumers as food products. In the processing of frozen food of agricultural products, the hot water treatments are commonly used as blanching, which is used for inactivation of microorganism and enzyme, at final stage of the process. PEF treatment is one of the candidates as alternating non-thermal methods for enzyme inactivation instead of thermal process. The PEF treatments for enzyme inactivation have been investigated by some researchers (Loey et al. 2002). Yeom and Zhang confirmed that the functions of enzymatic proteins was inactivated by PEF treatment at some optimized circuit parameters (Yeom and Zhang 2001). Vega-Mercado et al. also reported that the PEF parameters such as strength, pulse width, number of pulses and rise time of the pulse were mainly affected efficiency of enzyme inactivation. They also confirmed that inactivation of enzyme required generally more energy (i.e., PEF strength, pulse width and number of pulses) than that of microorganisms (Vega-Mercado et al. 1997). Castro et al. indicated that the pulse width of PEF was more dominant for inactivation of enzyme than PEF strength. They confirmed that the enzymic protein of alkaline phosphatase in milk

Fig. 85 Optical microscopic images of reaction inside the grape skin cell with and without PEF treatment

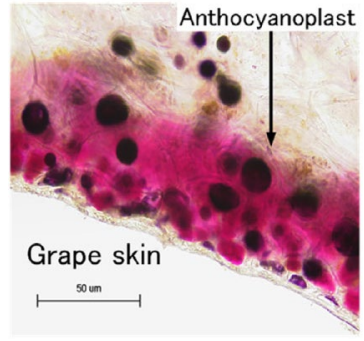

(a) Control

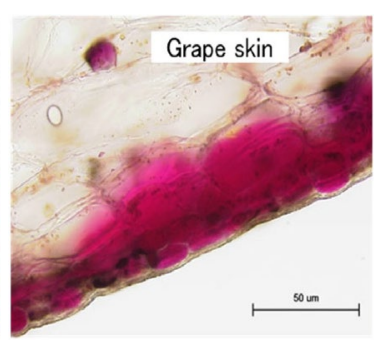

(b) $20 \mathrm{kV}(\Delta t=1020 \mathrm{~ns})$ 
Fig. 86 Increase rate of polyphenol extraction by PEF treatment for different pulse widths compared with water extraction in same temperatures (Nakagawa et al. 2013)

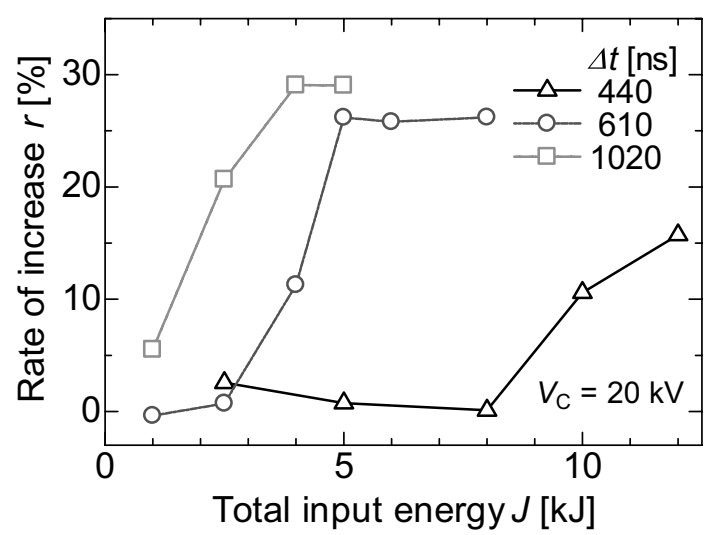

was inactivated with $65 \%$ at PEF of $22 \mathrm{kV} / \mathrm{cm}$ strength, $0.7 \mathrm{~ms}$ width and 70 pulses, whereas not inactivated at $26 \mathrm{kV} / \mathrm{cm}, 0.39 \mu \mathrm{sec}$ and 20 pulses (Castro et al. 2001). About the inactivation mechanism of enzyme by PEF treatment, Dong et al. pointed out that the conformational changes of enzymic proteins such as denaturation and aggregation caused the inactivation of enzymic proteins (Dong et al. 2020).

Guionet et al. reported the effect of PEF treatment on enzymic inactivation of $\alpha$-amylase. They developed pulse network (PFN) circuit for controlling pulse width and strength of PEF, which was applied between the electrodes with $4 \mathrm{~mm}$ gap in a cuvette. The curette was filled with $\alpha$-amylase solution, which was prepared by dissolving $25 \mathrm{mg} \alpha$-amylase in a solution consisted of $48 \mathrm{~mL}$ distilled water and $2 \mathrm{~mL}$ phosphate buffer. The results showed that the residual activity of $\alpha$-amylase decreased with PEF strength at the same input energy with $10 \mu$ s of pulse width, as shown in Fig. 87 (Guionet et al. 2021). This result indicates that the PEF strength affects the efficiency of protein conformational change strongly. They also confirmed the conformational change of proteins by PEF treatment, as shown in Fig. 88 (Guionet et al. 2021). The tertiary structure change of $\alpha$-amylase was monitored by fluorescence spectra at $280 \mathrm{~nm}$ wavelength of excitation light. The tertiary structure (mainly tryptophan; Trp) of $\alpha$-amylase also decreased with PEF strength at the same input energy. The enzymic active center of $\alpha$-amylase was the carboxyl terminus of tryptophan. $\alpha$-amylase commonly consists of three domains, which include several $\alpha$-helix and $\beta$-sheet secondary structures. PEF treatments affect mainly hydrogen bonds in secondary structure (i.e., $\alpha$-helix and $\beta$-sheet structure) and tertiary structure of the $\alpha$-amylase. It was also confirmed that the PEF and the heat treatments were different pathways for enzyme inactivation, as shown in Fig. 89 (Ohshima et al. 2021). They checked the aggregation of proteins after treatments by $\mathrm{PEF}$ with $12.5 \mathrm{kV} / \mathrm{cm}$ and heating up to $70{ }^{\circ} \mathrm{C}$. Bose treatments caused inactivation of $\alpha$-amylase in same level of lower than $0.01 \mathrm{U} / \mathrm{ml}$ in residual activity. The relative protein concentration after filtering with $0.22 \mu \mathrm{m}$ syringe filter of PEF treated $\alpha$-amylase solution is almost same level at control (without treatment), whereas the protein concentration of heat-treated $\alpha$-amylase solution decreases to approximately $37 \%$. The aggregation is confirmed to be only caused by heat treatment, because 
Fig. 87 Residual activity of $\alpha$-amylase for different strength of PEF at same input energy of $720 \mathrm{~J}$ (Guionet et al. 2021)

Fig. 88 Fluorescence spectra of $\alpha$-amylase at $280 \mathrm{~nm}$ wavelength of excitation light emission after PEF treatment for various strengths of PEF at same input energy of $720 \mathrm{~J}$ (Guionet et al. 2021)

Fig. 89 Residual protein concentration after PEF and heat treatments. PEF treatment at $12.5 \mathrm{kV} / \mathrm{cm}$ and heat treatment at $70{ }^{\circ} \mathrm{C}$ were the same treatment time of $40 \mathrm{~min}$ (Ohshima et al. 2021)
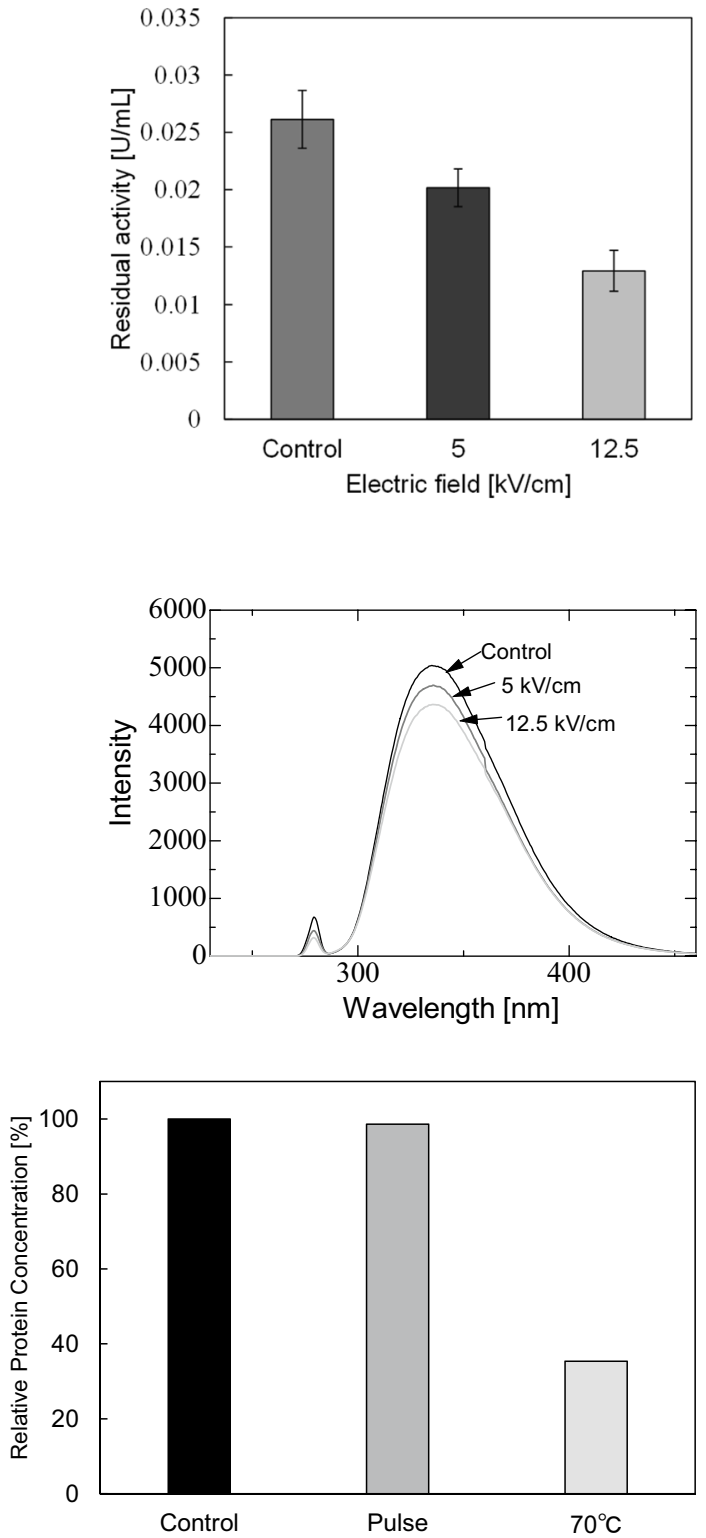

decrease of the relative protein is caused by aggregation. Therefore, PEF treatment mainly contributes to conformational change of the protein, resulting in enzymatic activity change (Guionet et al. 2021).

Refolding of denatured proteins has potential to recover the enzymic activity of denatured enzymes, and is important issue for effective enzyme usage in the food industry (Kubo et al. 1993). The PEF treatment induces the conformational change of protein. Therefore, the PEF treatment is a candidate of novel method 
to refold the denatured enzymes. Ohshima et al. confirmed that the activity of six kinds of enzyme increased with rate of $105 \%-120 \%$ by PEF treatment. They concluded that this enzyme activations were caused by protein conformational change or enzyme hydration (Ohshima et al. 2007). They also reported that the activity of thermal denatured peroxidase was recovered to $60 \%$ of initial activity by PEF treatment with $12 \mathrm{kV} / \mathrm{cm}$ strength, $50 \mathrm{~Hz}$ repetition rate and $30 \mathrm{~s}$ exposure, whereas the enzymic activity was recovered to only $40 \%$ of the initial activity at spontaneous refolding of the enzyme. However, the activity of thermal denatured lactate dehydrogenase (LDH) decreased by PEF treatment, which suggested that further inactivation was caused by PEF applying to the thermal denatured LDH (Ohshima et al. 2007). Therefore, the effect of PEF treatment on refolding of the denatured proteins depends on protein structure, i.e., type of enzyme.

$\mathrm{PEF}$ treatment is a relatively low-temperature process compared with heat treatment, and is effective for inactivation of not only foodborne and food spoilage bacteria but also enzymic proteins without degradation of nutritional and sensory properties (Yeom et al. 2000; Elez-Martínez et al. 2006). However, sometimes, the PEF treatment alone for enzyme inactivation requites long period of process time or large electrical input power. Shamsi et al. proposed the combination with moderate heat treatment and PEF treatment to enhance the efficiency of inactivation of enzymes and bacteria in whole milk (Shamsi et al. 2008). Ho et al. confirmed that an inactivation of enzymes generally required more input energy in PEF treatment compared with the case of inactivation of microorganisms (Ho et al. 1997). Agcam et al. conducted that the inactivation of pectin methyl esterase (PME) in orange juice by PEF treatment. The inactivation of PME was significantly induced at large input energy of PEF irradiation to the PME solution. A kinetic model was also proposed for estimating efficiency of PME inactivation. In the model, the inactivation efficiency was expressed as a function of PEF treatment conditions such as input power and treatment time. The kinetic model was confirmed to be effective for estimation of the reaction rate and the time required for a $90 \%$ inactivation (Agcam et al. 2014). Sharma et al. reported the effect of PEF treatment on inactivation of four indigenous enzymes in whole milk. The lipase, plasmin, xanthine oxidase and alkaline phosphatase were used as specimens of indigenous enzymes in raw milk. The experimental results showed that the enzymic activities of plasmin, xanthine oxidase and lipolytic decreased with 12,32 , and $82 \%$ reducing rate, respectively, compared with the raw whole milk by PEF treatment with $26.1 \mathrm{kV} / \mathrm{cm}$ strength at $34 \mu \mathrm{s}$ pulse width. When the strength of PEF increased to greater than $20.7 \mathrm{kV} / \mathrm{cm}$ for $34-101 \mu \mathrm{s}$, the enzymic activity of alkaline phosphatase was reduced to a comparable to thermal treatments. These results indicated that thermal effects also contribute to inactivation of bacteria and enzymes along with the PEF treatments (Sharma et al. 2014b).

\section{Summary}

The pulsed power technologies including high-electric field and time-modulated non-thermal plasmas have a potential to contribute the improvement in agriculture and food processing. This paper treated the pulsed power including high-electric 
field and time-modulated non-thermal plasmas and its applications in agriculture and food processing with introducing many pioneering and excellent works investigated by many researchers. The pulsed power technologies are based on energy compressing temporally. In general, the pulsed power technologies treat the wide time range from picoseconds to milliseconds owing to the variety of applications such as light sources, charged particle accelerations, nuclear fusions, shockwaves, etc. In this paper, the pulsed power in time ranges between nanoseconds and several tens microsecond was mainly treated because typical time constant for charging time of cell membrane is around $1 \mathrm{~ms}$, which is corresponding to $1 \mathrm{MHz}$ of $\mathrm{AC}$ frequency.

The direct capacitive discharge is commonly used as simple pulsed power system with a single switch. An association of circuits to circumvent the still voltage and current limitation of semiconductors are also frequently used to generate an intense high voltage, such as and inductively multiplied circuit and a Marx generator. The pulse transmission lines and pulse-forming network to generate are used to generate a square shape waveform transferred to the loads. The intense electric field can induce the biological effect such as electroporation. Moreover, the electric field accelerates charged particles by the Coulomb force, following that, an electron avalanches are formed and developed to orientation of the electric field. The electron avalanches work as trigger of a discharge onset. After the discharge onset, the discharge such as streamer corona generates and propagates toward the electric field. The electric field distribution between the electrodes changes from that expressed by Laplace equation to that containing electric field produced with charged particles of discharge plasma. The strong electric field of the discharge plasma such as vicinity of streamer head can cause biological effect. Moreover, the discharge plasma includes reactive species which also can cause biological reactions on the plants. These two effects of the discharge plasma on the living organisms such as plants causes in same time, as the result, the synergistic phenomena are sometimes induced. Therefore, it is important to clarify the mechanism of the effect of pulsed discharge on plants through the analysis of gene expression and enzyme activation, etc.

In the pre-harvest phase of agriculture, the promotion of plant growth is important issue for improving crop production. The pulsed power technologies can be used for improvement of crop production via promotion of plant seed germination, seedling and plant growth enhancement, inactivation of pathogenic bacteria in cultivation media, and promotion of fruit body formation. The intense PEF and the timemodulated discharge plasmas can break a dormancy and activate plant hormone related to seed germination promotion. The pulse plasmas contribute plant growth enhancement through the nitrogen fixation in the cultivation media or inactivation of pathogenic bacteria through the oxidation reaction. The intense PEF works as direct stimuli which activates metabolism of plants such as photosynthesis. The intense PEF also works as direct stimuli of induction of the fruit body formation. This technique can be used to improving yielding rage of mushroom.

In the post-harvest phase of agriculture, the keeping quality of agricultural products is important for effective food supply chain. The pulsed power technologies can also contribute a modern food supply chain through maintaining quality 
of agricultural products through decontaminating from ambient air in the storage containers. The pulsed plasmas also contribute the keeping quality of vegetables and fruits by decomposing ripening acceleration plant hormone emitted by some products. In the food processing phase, the intense PEF can be used as non-thermal pasteurization through the electroporation phenomena. This phenomenon can be used to extract intracellular contents such as juice, nutritional agent and health-promoting agent in food processes. PEF can also contribute enlarge the preservation period through the inactivation of enzyme and microorganism based of protein conformational change.

The pulsed power applications in agriculture and food processing are new research field and still mainly in experimental stages for developing to the industrial use. For expanding the applications from laboratories to industries, there are some issues that must be addressed. One important issue is how to effectively apply the new technologies to the modern food supply chain to achieve a comprehensive sustainable system, which includes to be economical and low in working labor. To address the issues, the collaborations among the academic in many different specialties, government staffs and industries are one of the key factors. The government staffs of agriculture and food division can make a blueprint of novel and global food supply chain with considering newly developed the pulsed power and plasma applications. The industries can work to commercialize the concept and apparatus of the pulsed power applications. In addition, it is necessary to design the regulations for the new technologies. The collaboration among the government, academia and industries is also important to design the effective regulation. Moreover, the agriculture is now shifting from soil-based cultivation to plant factory which is a closed growing system with artificially controlling light, temperature, moisture, and carbon dioxide concentrations. Therefore, new technologies of the pulsed power application need to be considered for installation in plant factories. The clarifying the mechanism biologically and electrically for each application is also important to develop the effective system. In this issue, the collaborations among specialists and researchers in varieties of academic fields are also very important for realization and development of these applications.

Acknowledgements The authors would like to thank the Japan Society for financial support of a Grantin-Aid for Scientific Research Foundations S (Grant no. 19H05611). The authors would like to thank Prof. M. Shiratani of Kyusyu University, Prof. T. Uchino of Kyusyu University, Prof. K. Koga of Kyusyu University and Dr. C. Yuan of Iwate University for their valuable comments and discussions.

Open Access This article is licensed under a Creative Commons Attribution 4.0 International License, which permits use, sharing, adaptation, distribution and reproduction in any medium or format, as long as you give appropriate credit to the original author(s) and the source, provide a link to the Creative Commons licence, and indicate if changes were made. The images or other third party material in this article are included in the article's Creative Commons licence, unless indicated otherwise in a credit line to the material. If material is not included in the article's Creative Commons licence and your intended use is not permitted by statutory regulation or exceeds the permitted use, you will need to obtain permission directly from the copyright holder. To view a copy of this licence, visit http://creativecommons.org/licen ses/by/4.0/. 


\section{References}

F.B. Abeles, P.W. Morgan, M.E. Saltveit Jr., Ethylene in plant biology (Academic, London, 1992), p. 182

E. Agcam, A. Akyıldı, G.A. Evrendilek, Effects of PEF and heat pasteurization on PME activity in orange juice with regard to a new inactivation kinetic model. Food Chem. 165, 70-76 (2014)

H. Akiyama, R. Heller, Bioelectrics (Springer, Tokyo, 2017)

C. Alonso, P.C. Raynor, P.R. Davies, R.B. Morrison, Evaluation of an electrostatic particle ionization technology for decreasing airborne pathogens in pigs. Aerobiologia 32, 405-419 (2016)

C. Alves Jr., J.O. Vitoriano, D.L.S. Silva, M.L. Farias, N.B.L. Dantas, Water uptake mechanism and germination of Erythrina velutina seeds treated with atmospheric plasma. Sci. Rep. 6, 3722 (2016)

D.R.B. Aroush, S. Yehudai-Resheff, K. Keren, Electrofusion of giant unilamellar vesicles to cells. Methods Cell Biol. 125, 409-422 (2015)

K. Asami, Cell electrofusion in centrifuged erythrocyte pellets assessed by dielectric spectroscopy. J. Membr. Biol. 249, 31-39 (2016)

P. Attri, Y.H. Kim, D.H. Park, J.H. Park, Y.J. Hong, H.S. Uhm, K.-N. Kim, A. Fridman, E.H. Choi, Generation mechanism of hydroxyl radical species and its lifetime prediction during the plasmainitiated ultraviolet (UV) photolysis. Sci. Rep. 5, 9332 (2015)

P. Attri, K. Koga, T. Okumura, M. Shiratani, Impact of atmospheric pressure plasma treated seeds on germination, morphology, gene expression and biochemical responses. Jpn. J. Appl. Phys. 60, 040502 (2021)

F.J. Barba, N. Grimi, E. Vorobiev, New approaches for the use of non-conventional cell disruption technologies to extract potential food additives and nutraceuticals from microalgae. Food Eng. Rev. 7, 45-62 (2014)

F.J. Barba, O. Parniakov, S.A. Pereira, A. Wiktor, N. Grimi, N. Boussetta, J.A. Saraiva, J. Raso, O. Martin-Belloso, D. Witrowa-Rajchert, N. Lebovka, E. Vorobiev, Current applications and new opportunities for the use of pulsed electric fields in food science and industry. Food Res. Int. 77, 773-798 (2015)

G.V. Barbosa-Canovas, B. Altunakar, Pulsed electric fields processing of foods: an overview, in Pulsed Electric Field Technology for Food Industry: Fundamentals and Applications. ed. by J. Raso, V. Heinz (Springer, New York, 2006), pp. 153-194

P. Barman, R. Bhattacharya, Impact of electric and magnetic field exposure on young plants-a review. Int. J. Curr. Res. Aca. Rev. 4, 182-192 (2016)

F. Basty, Essaisd'electroculturetentesa Angers en. Bui. Soc. Etudes Sci. Angers Aim. 37, 87-92 (1908)

G. Beccaria, The manuscripts of correspondent of Benjamin Franklin. Proc. Am. Philos. Soc. 96, 406$411(1775)$

I. Bekard, D.E. Dunstan, Electric field induced changes in protein conformation. Soft Matter 10, 431-437 (2014)

M. Berthelot, Recherchesnouvelles sur la fixation de 1 Azote par la terrevegetale. Influence de l'electricite. Compt. Rend. Acad. Sci. 109, 281-287 (1889)

M. Bertholon, De l'electricit des vrgrtaux, Didot Jeune, (Paris, 1783) pp. 1742-1800

J.D. Black, F.R. Forsyth, D.S. Fensom, R.B. Ross, Electrical stimulation and its effects on growth and ion accumulation in tomato plants. Can. J. Bot. 49, 1809-1815 (1971)

H. Bluhm, Pulsed Power System (Springer, Berlin, 2006)

Board of Agriculture and Fisheries, Reports 1-18 of the Electro-Culture Committee, Food and Fisheries, Great Westminster House. Horseferry Road. London (1918-1937)

P. Boonkorn, H. Gemma, S. Sugaya, S. Setha, J. Uthaibutra, K. Whangchai, Impact of high-dose, short periods of ozone exposure on green mold and antioxidant enzyme activity of tangerine fruit Postharvest. Biol. Technol. 67, 25-28 (2012)

J.C. Bose, Comparative electro-physiology. A physico-physiological study (Longmans Green \& Co. London, New York, 1907)

R. Bovelli, A. Bennici, Stimulation of germination, callus growth and shoot regeneration of Nicotiana tabacum L. by pulsing electromagnetic fields (PEMF). Adv. Hortic. Sci. 14, 3-6 (2000)

N.S.J. Braithwaite, Introduction to gas discharges. Plasma Sources Sci. Technol. 9, 517-527 (2000)

L. Brauner, Untersuchungenüber das geoelektrischePhänomen. Jahrb. Wiss. Bot. 66, 381-428 (1927)

O.A. Brown, R.B. Stone Jr., H. Andrews, Methods and equipment for low energy irradiation of seeds. Agric. Eng. 38, 666-669 (1957) 
P. Bruggeman, C. Leys, Non-thermal plasmas in and in contact with liquids. J. Phys. D Appl. Phys. 42, 053001 (2009)

D. Butscher, H. Van Loon, A. Waskow, P.R. von Rohr, M. Schuppler, Plasma inactivation of microorganisms on sprout seeds in a dielectric barrier discharge. Int. J. Food Microbiol. 238, 222-232 (2016)

Z. Cai, H. Riedel, N.M.M.T. Saw, O. Kütük, I. Mewis, H. Jäger, D. Knorr, I. Smetanska, Effects of pulsed electric field on secondary metabolism of Vitis vinifera L. cv. Gamay Fréaux suspension culture and Exudates. Appl. Biochem. Biotechnol. 164, 443-453 (2011)

A.J. Castro, B.G. Swanson, G.V. Barbosa-Cánovas, Q.H. Zhang, Pulsed electric fields modification of milk alkaline phosphatase activity, in Pulsed Electric Fields in Food Processing. ed. by G.V. Barbosa-Cánovas, Q.H. Zhang (Technomic Publishing, Lancaster, 2001), pp. 65-82

A.J. Castro, G.V. Barbosa-Cánovas, B.G. Swanson, Microbial inactivation of foods by pulsed electric field. J. Food Process. Preserv. 17, 47-73 (2007)

J.S. Chang, P.A. Lawless, T. Yamamoto, Corona discharge processes. IEEE Trans. Plasma Sci. 19, 1152$1166(1991)$

S. Chavadej, K. Saktrakool, P. Rangsunvigit, L.L. Lobban, T. Sreethawong, Oxidation of ethylene by a multistage corona discharge system in the absence and presence of $\mathrm{Pt} / \mathrm{TiO}_{2}$. Chem. Eng. J. 132, 345-353 (2007)

A. Choudhary, A. Kumar, N. Kaur, ROS and oxidative burst: roots in plant development. Plant Divers. 42, 33-43 (2020)

W.G. Clark, Polar transport of auxin and electrical polarity in coleoptile of Avena. Plant Physiol. 12, 409-440 (1937)

O. Cregenzán-Alberti, R.M. Halpin, P. Whyte, J. Lyng, F. Noci, Suitability of ccRSM as a tool to predict inactivation and its kinetics for Escherichia coli, Staphylococcus aureus and Pseudomonas fluorescens in homogenized milk treated by manothermosonication (MTS). Food Control 39, 41-48 (2014)

O. Cregenzan-Alberti, R.M. Halpin, P. Whyte, J.G. Lyng, F. Noci, Study of the suitability of the central composite design to predict the inactivation kinetics by pulsed electric fields (PEF) in Escherichia coli, Staphylococcus aureus and Pseudomonas fluorescens in milk. Food Bioprod. Process. 95, 313-322 (2015)

P.J. Cullen, J. Lalor, L. Scally, D. Boehm, V. Milosavljević, P. Bourke, K. Keener, Translation of plasma technology from the lab to the food industry. Plasma Process Polym. 15, e1700085 (2018)

L. Degutytė-Fomins, G. Paužaitė, R. Žūkienė, V. Mildažienė, K. Koga, M. Shiratani, Relationship between cold plasma treatment-induced changes in radish seed germination and phytohormone balance. Jpn. J. Appl. Phys. 59, SH1001 (2020)

Q. Deng, K.G. Zinoviadou, C.M. Galanakis, V. Orlien, N. Grimi, E. Vorobiev, N. Lebovka, F.J. Barba, The effects of conventional and non-conventional processing on glucosinolates and its derived forms, isothiocyanates: extraction, degradation, and applications. Food Eng. Rev. 7, 357-381 (2014)

F. Ding, J.M. Borreguero, S.V. Buldyrey, H.E. Stanley, N.V. Dokholyan, Mechanism for the a-helix to b-hairpin transition. Proteins 53, 220 (2003)

D. Dobrin, M. Magureanu, N.B. Mandache, M.D. Ionita, The effect of non-thermal plasma treatment on wheat germination and early growth. Innov. Food Sci. Emerg. Technol. 29, 255-260 (2015)

M. Dong, Y. Xu, Y. Zhang, M. Han, P. Wang, X. Xu, G. Zhou, Physicochemical and structural properties of myofibrillar proteins isolated from pale, soft, exudative (PSE)-like chicken breast meat: effects of pulsed electric field (PEF). Innov. Food Sci. Emerg. Technol. 59, 102277 (2020)

C.S. Dorchester, The effects of electric current on certain crop plants. Iowa State College of Agri. and Mechanical Arts. Res. Bui.1-210 (1937)

A.E. Dubinov, E.M. Lazarenko, V.D. Selemir, Effect of glow discharge air plasma on grain crops seed. IEEE Trans. Plasma Sci. 28, 180-183 (2000)

K. Dymek, P. Dejmek, V. Panarese, A.A. Vicente, L. Wadsö, C. Finnie, G.F. Gómez, Effect of pulsed electric field on the germination of barley seeds. Food Sci. Technol. 47, 161-166 (2012)

K. Ebihara, H.D. Stryczewska, F. Mitsugi, T. Ikegami, T. Sakai, J. Pawlat, S. Teii, Recent development of ozone treatment for agricultural soil sterilization and biomedical prevention. Przeglad Elektrotechniczny 88, 92-94 (2012)

J. Ehlbeck, U. Schnabel, M. Polak, J. Winter, Th. von Woedtke, R. Brandenburg, T. von dem Hagen, K.-D. Weltman, Low temperature atmospheric pressure plasma sources for microbial decontamination. J. Phys. D Appl. Phys. 44, 053002 (2011) 
C.J. Eing, S. Bonnet, M. Pacher, H. Puchta, W. Frey, Effects of nanosecond pulsed electric field exposure on Arabidopsis thaliana. IEEE Trans. Dielectr. Electr. Insul. 16, 1322-1328 (2009)

P. Elez-Martínez, I. Aguiló-Aguayo, O. Martin-Belloso, Inactivation of orange juice peroxidase by highintensity pulsed electric fields as influenced by process parameters. J. Sci. Food Agric. 86, 71-81 (2006)

F. Elfving, UebereineWirkung des galvanischenStromes auf wachsendeWurzeln. In Bot. Ztg. Jahrg. 40, 257-264 (1882)

B. Eliasson, U. Kogelschatz, Nonequilibrium volume plasma chemical processing. IEEE Trans. Plasma Sci. 19, 1063-1077 (1991a)

B. Eliasson, U. Kogelschatz, Modeling and applications of silent discharge plasmas. IEEE Trans. Plasma Sci. 19, 309-323 (1991b)

A. Fernández, A. Thompson, The inactivation of Salmonellaby cold atmospheric plasma treatment. Food Res. Int. 45, 678-684 (2012)

M. Fincan, P. Dejmek, In situ visualization of the effect of a pulsed electric field on plant tissue. J. Food Eng. 55, 223-230 (2002)

A. Fridman, A. Chirokov, A. Gutsol, Non-thermal atmospheric pressure discharges. J. Phys. D Appl. Phys. 38, R1-24 (2005)

M.J. Gallagher, N. Vaze, S. Gangoli, V.N. Vasilets, A.F. Gutsol, T.N. Milovanova, S. Anandan, D.M. Murasko, A.A. Fridman, Rapid inactivation of airborne bacteria using atmospheric pressure dielectric barrier grating discharge. IEEE Trans. Plasma Sci. 35, 1501-1510 (2007)

G. Gassner, ZurFrage der Elektrokultur. Ber. Deut. Bot. Gesell. 25, 26-38 (1907)

G. Gassner, PflanzenphysiologischeFragen der Elektrokultur. In Mitt. Deut. Landw. Gesell Jahrg. 24, 5-7 (1909)

L.F. Gaunt, C.B. Beggs, G.E. Georghiou, Bactericidal action of the reactive species produced by gasdischarge nonthermal plasma at atmospheric pressure: a review. IEEE Trans. Plasma Sci. 34, 1257-1269 (2006)

V.I. Gibalov, G.J. Pietsch, The development of dielectric barrier discharges in gas gaps and on surfaces. J. Phys. D Appl. Phys. 33, 2618-2636 (2000)

L. Grandeau, De l'influence de l'electriciteatmospherique sur la nutrition des plantes. In Compt. Rend. Acad. Sci. 87, 60-62 (1878)

D.B. Graves, The emerging role of reactive oxygen and nitrogen species in redox biology and some implications for plasma applications to medicine and biology. J. Phys. D Appl. Phys. 45, 263001 (2012)

A. Guionet, T. Fujiwara, H. Sato, K. Takahashi, K. Takaki, M. Matsui, T. Tanino, T. Ohshima, Pulsed electric fields act on tryptophan to inactivate $\alpha$-amylase. J. Electrostat. 112, 103597 (2021)

S. Hamada, S. Ezaki, K. Hayashi, K. Toko, K. Yamafuji, Electric current precedes emergence of a lateral root in higher plants. Plant Physiol. 100, 614-619 (1992)

D. Hamanaka, N. Norimura, N. Baba, K. Mano, M. Kakiuchi, F. Tanaka, T. Uchino, Surface decontamination of fig fruit by combination of infrared radiation heating with ultraviolet irradiation. Food Control 22, 375 (2011)

T. Han, H.R. An, G. Mainelis, Performance of an electrostatic precipitator with superhydrophobic surface when collecting airborne bacteria. Aerosol Sci. Technol. 6826, 339-348 (2010)

X. Han, W. Cantrell, E.E. Escobar, S. Ptasinska, Plasmid DNA damage induced by helium atmospheric pressure plasma jet. Eur. Phys. J. D 68, 46 (2014)

F.X. Hart, R.S. Schottenfeld, Evaporation and plant damage in electric fields. Int. J. Biometeorol. 23, 63-68 (1979)

H. Hashizume, H. Kitano, H. Mizuno, A. Abe, G. Yuasa, S. Tohno, H. Tanaka, K. Ishikawa, S. Matsumoto, H. Sakakibara, S. Nikawa, M. Maeshima, M. Mizuno, M. Hori, Improvement of yield and grain quality by periodic cold plasma treatment with rice plants in a paddy field. Plasma Process Polym. 18, e2000181 (2020)

N. Hayashi, R. Ono, M. Shiratani, A. Yonesu, Antioxidative activity and growth regulation of Brassicaceae induced by oxygen radical irradiation. Jpn. J. Appl. Phys. 54, 06 GD01 (2015)

M. Henselová, L. Slováková, M. Martinka, A. Zahoranová, Growth, anatomy and enzyme activity changes in maize roots induced by treatment of seeds with low temperature plasma. Biologia $\mathbf{6 7}$, 490-497 (2012)

P.K. Hepler, R. Wayne, Calcium and plant cell development. Ann. Rev. Plant Physiol. 38, 397-439 (1985)

J.T. Herron, D.S. Green, Chemical kinetics database and predictive schemes for nonthermal humid air plasma chemistry. Part II. Neutral species reactions. Plasma Chem. Plasma Process. 21, 459-481 (2001) 
N. Higinbotham, Electro potentials of plant cells. Ann. Rev. Plant Physiol. 24, 25-46 (1973)

A.M. Hirst, M.S. Simms, V.M. Mann, N.J. Maitland, D.O. Connell, F.M. Frame, Low-temperature plasma treatment induces DNA damage leading to necrotic cell death in primary prostate epithelial cells. J. Cancer. 112, 1536-1545 (2015)

S.Y. Ho, G.S. Mittal, J.D. Cross, Effects of high field electric pulses on the activity of selected enzymes. J. Food Eng. 31, 69-84 (1997)

L' Holubová, S. Kyzek, I. Ďurovcová, J. Fabová, E. Horváthová, A. Ševčovičová, E. Gálová, Non-thermal plasma-a new green priming agent for plants? Int. J. Mol. Sci. 21, 9466 (2020)

R. Huang, S. Sukprakarn, L. Phavaphutanon, S. Juntakool, C. Chaikul, A comparison of electric field treatments to hydropriming on cucumber seed germination enhancement. Kasetsart J. Nat. Sci. 40, 559-565 (2006)

Y. Huang, S. Li, Q. Zheng, X. Shen, S. Wang, P. Han, Z. Liu, K. Yan, Recent progress of dry electrostatic precipitation for PM2.5 emission control from coal-fired boilers. Int. J. Plasma Environ. Sci. Technol. 9, 69-95 (2015)

T. Huiskamp, Nanosecond pulsed streamer discharges. Part I: generation, source-plasma interaction and energy-efficiency optimization. Plasma Sources Sci. Technol. 29, 023002 (2020)

H. Hülsheger, J. Potel, E.G. Niemann, Killing of bacteria with electric pulses of high electric field strength”. Radiat. Environ. Biophys. 20, 53-65 (1981)

J. Hyuk, I. Han, H.K. Baik, M.H. Lee, D.W. Han, J.C. Park, I.S. Lee, K.M. Song, Y.S. Lim, Analysis of sterilization effect by pulsed dielectric barrier discharge. J. Electrostat. 64, 17-22 (2006)

T. Idei, N. Yoshizawa, M. Tako, Effects of electric shocks to the bed-logs of Lentinus edodes on fruitingbody production. Bull. Utsunomiya Univ. for. 24, 23-38 (1988)

F. Islam, S. Ohga, The response of fruit body formation on Tricholoma matsutake in situ condition by applying electric pulse stimulator. ISRN Agron. 462724, 1-6 (2012)

M. Ito, T. Ohta, M. Hori, Plasma agriculture. J. Korean Phys. Soc. 60, 937-943 (2012)

M. Ito, H. Hashizume, J.-S. Oh, K. Ishikawa, T. Ohta, M. Hori, Inactivation mechanism of fungal spores through oxygen radicals in atmospheric pressure plasma. Jpn. J. Appl. Phys. 60, 010503 (2021)

S.H. Ji, K.-H. Choi, A. Pengkit, J.S. Im, J.S. Kim, Y.H. Kim, Y. Park, E.J. Hong, S.K. Jung, E.-H. Choi, G. Park, Effects of high voltage nanosecond pulsed plasma and micro DBD plasma on seed germination, growth development and physiological activities in spinach. Arch. Biochem. Biophys. 605, 117-128 (2016)

J. Jiang, Y. Lu, J. Li, L. Li, X. He, H. Shao, Y. Dong, Effect of seed treatment by cold plasma on the resistance of tomato to Ralstonia solanacearum. PLoS ONE 9, e97753 (2014a)

C. Jiang, R.V. Davalos, J.C. Bischof, A review of basic to clinical studies of irreversible electroporation therapy. IEEE Trans. Biomed. Eng. 62, 4-20 (2014b)

Z. Jiang, L. You, W. Dou, T. Sun, P. Xu, Effects of an electric field on the conformational transition of the protein: a molecular dynamics simulation study. Polymers 11, 282 (2019)

K. Kadowaki, N. Kurisaka, Stimulation and inhibition of Arabidopsis seed germination with repetitive barrier discharges produced by polarity-reversed voltage pulses. Trans. Inst. Electr. Eng. Jpn. A 133, 38-43 (2013)

I.M. Kalinina, K. Vladimir, M. Iva, T. Nørrelykke, Cell polarity: which way to grow in an electric field? Curr. Biol. 20, 355-356 (2010)

S. Kaneko, M. Yamamoto, Y. Nakashima, Y. Jitsufuji, Studies on electrical stimulation on Lentinula edodes bed log. Bull. Fukuoka-Ken for. Exp. Stn. 33, 1-33 (1987)

S. Katsuki, N. Nomura, H. Koga, H. Akiyama, I. Uchida, S. Abe, Biological effects of narrow band pulsed electric fields. IEEE Trans. Dielectr. Electr. Ins. 14, 663-668 (2007)

C.R. Keller, Plant treating system, United States Patent M 3,120, 72241 Palm Court, Santa Paula, Calif. (1966)

N. Khamsen, D. Onwimol, N. Teerakawanich, S. Dechanupaprittha, W. Kanokbannakorn, K. Hongesombut, S. Srisonphan, Rice (Oryza sativa L.) seed Sterilization and germination enhancement via atmospheric hybrid nonthermal discharge plasma. ACS Appl. Mater. Interfaces 8, 19268-19275 (2016)

G.P. Kharel, F. Hashinaga, Effect of high electric field on shelf life of strawberries. Food Sci. Technol. Int. 2, 198-202 (1996)

I. Kieft, E. van der Laan, E. Stoffels, Electrical and optical characterization of the plasma needle. New J. Phys. 6, 149 (2004)

A. Kilonzo-nthenge, S. Liu, S. Yannam, A. Patras, Atmospheric cold plasma inactivation of Salmonella and Escherichia coli on the surface of golden delicious apples. Front. Nutr. 5, 120 (2018) 
N. Kitajima, K. Ueda, T. Ohshima, M. Sato, Development of textile electrode for microbial inactivation with pulsed electric field. Text. Res. J. 77, 528-534 (2007)

S. Kitazaki, T. Sarinont, K. Koga, N. Hayashi, M. Shiratani, Plasma induced long-term growth enhancement of Raphanus sativus L. using combinatorial atmospheric air dielectric barrier discharge plasmas. Curr. Appl. Phys. 14, S149-153 (2014)

K. Koga, T. Sarinont, T. Amano, H. Seo, N. Itagaki, N. Hayashi, M. Shiratani, Simple method of improving harvest by nonthermal air plasma irradiation of seeds of Arabidopsis thaliana (L.). Appl. Phys. Express 9, 016201 (2016)

U. Kogelschatz, Dielectric-barrier discharges: their history, discharge physics, and industrial applications. Plasma Chem. Plasma Process. 23, 1-46 (2003)

S. Koide, A. Nakagawa, K. Omoe, K. Takaki, T. Uchino, Physical and microbial collection efficiencies of an electrostatic precipitator for abating airborne particulates in postharvest agricultural processing. J. Electrost. 71, 734-738 (2013)

J.F. Kolb, A.M. Mattson, C.M. Edelblute, X. Hao, M.A. Malik, L.C. Heller, Cold DC-operated air plasma jet for the inactivation of infectious microorganisms. IEEE Trans. Plasma Sci. 40, 3007-3026 (2012)

S. Kotaka, A.P. Krueger, Air ion effects on RNAse activity in green barley leaves. Int. J. Biometeor. 16, 1-11 (1972)

S. Kotaka, A.P. Krueger, P.C. Andriese, The effect of air ions on light-induced swelling and dark-induced shrinking of isolated chloroplasts. Int. J. Biometeor. 12, 85-92 (1968)

T. Kotnik, W. Frey, M. Sack, S.H. Meglic, M. Peterka, D. Miklavcic, Electroporation-based applications in biotechnology. Trends Biotechnol. 33, 480-488 (2015)

M. Koubaa, E. Rosello-Soto, J. Sic-Zlabur, A. Rezek-Jambrak, M. Brncic, N. Grimi, F.J. Barba, Current and new insights in the sustainable and green recovery of nutritionally valuable compounds from Stevia rebaudiana Bertoni. J. Agric. Food Chem. 63, 6835-6846 (2015)

A.P. Krueger, S. Kotaka, P.C. Andriese, Some observations on the physiological effects of gaseous ions. Int. J. Biometeor. 6, 33-48 (1962)

A.P. Krueger, S. Kotaka, P.C. Andriese, A study of the mechanism of air-ion-induced growth stimulation in Hordeum vulgaris. Int J Biometeorol 7, 17-25 (1963)

A.P. Krueger, S. Kotaka, P.C. Andriese, Studies on air-ion enhanced iron chlorosis. I. Active and residual iron. Int. J. Biometeorol. 8, 5-16 (1964)

A.P. Krueger, A.E. Strubbe, M.G. Yost, E.J. Reed, Electric fields, small air ions and biological effects. Int. J. Biometeorol. 22, 202-212 (1978)

T. Kubo, T. Mizobata, Y. Kawata, Refolding of yeast enolase in the presence of the chaperonin GroE. The nucleotide specificity of GroE and the role of GroES. J. Biol. Chem. 268, 19346-19351 (1993)

S. Kudo, S. Mitobe, Y. Yoshimura, Electric stimulation multiplication of Lentinulus edodes. J. Inst. Electrostat. Jpn. 23, 186-190 (1999)

E. Kuffel, W.S. Zaengl, D. Kuffel, High voltage engineering: fundamentals, 2nd edn. (Elsevier, Oxford, 2000)

M. Laroussi, X. Lu, M. Keidar, Perspective: the physics, diagnostics, and applications of atmospheric pressure low temperature plasma sources used in plasma medicine. J. Appl. Phys. 122, 020901 (2017)

R. Laurita, N. Contaldo, Y. Zambon, A. Bisag, A. Canel, M. Gherardi, G. Laghi, A. Bertaccini, V. Colombo, The use of plasma-activated water in viticulture: induction of resistance and agronomic performance in greenhouse and open field. Plasma Process Polym. 18, e2000206 (2021)

J. Lehr, P. Rpn, Foundations of pulsed power technology (Wiley, Hobson, 2017)

M.S. Lemström, Electricity in agriculture and horticulture (Franklin Classics Trade Press, London and New York, 1904), pp. 1-72

L. Li, J. Jiang, J. Li, M. Shen, X. He, H. Shao, Y. Dong, Effects of cold plasma treatment on seed germination and seedling growth of soybean. Sci. Rep. 4, 5859 (2014)

L. Li, J. Li, M. Shen, J. Hou, H. Shao, Y. Dong, J. Jiang, Improving seed germination and peanut yields by cold plasma treatment. Plasma Sci. Technol. 18, 1027-1033 (2016)

Y. Liang, Y. Wu, K. Sun, Q. Chen, F. Shen, J. Zhang, M. Yao, T. Zhu, J. Fang, Rapid inactivation of biological species in the air using atmospheric pressure nonthermal plasma. Environ. Sci. Technol. 46, 3360-3368 (2012)

C.-M. Liu, Y. Nishida, K. Iwasaki, K. Ting, Prolonged preservation and sterilization of fresh plants in controlled environments using high-field plasma IEEE Trans. Plasma Sci. 39, 717-724 (2011) 
A. Van Loey, B. Verachtert, M. Hendrickx, Effects of high electric field pulses on enzymes. Trends Food Sci. Technol. 12, 94-102 (2002)

J.J. Lowke, R. Morrow, Theoretical analysis of removal of oxides of sulphur and nitrogen in pulsed operation of electrostatic precipitators. IEEE Trans. Plasma Sci. 23, 661-671 (1995)

H. Lu, S. Patil, K.M. Keener, P.J. Cullen, P. Bourke, Bacterial inactivation by high-voltage atmospheric cold plasma: influence of process parameters and effects on cell leakage and DNA. J. Appl. Microbiol. 116, 784-794 (2014)

P. Lules, E. Dolezalova, I. Sisrova, M. Clupek, Aqueous phase chemistry and bactericidal effects from an air discharge plasma in contact with water: evidence for the formation of peroxynitrite through a pseudo-second-order post-discharge reaction of $\mathrm{H}_{2} \mathrm{O}_{2}$ and $\mathrm{HNO}_{2}$. Plasma Sources Sci. Technol. 23, 015019 (2014)

C. Lull, H.J. Wichers, H.F.J. Savelkoul, Antiinflammatory and immunomodulating properties of fungal metabolites. Mediat. Inflamm. 2005(2), 63-80 (2005)

S. Mahnič-Kalamiza, E. Vorobiev, D. Miklavčič, Electroporation in food processing and biorefinery. J. Membr. Biol. 247, 1279-1304 (2014)

M. Mainelis, K. Willeke, A. Adhikari, T. Reponen, S.A. Grinshpun, Design and collection efficiency of a new electrostatic precipitator for bioaerosol collection. Aerosol Sci. Technol. 36, 10731085 (2002a)

G. Mainelis, A. Adhikari, K. Willeke, S. Lee, Collection of airborne microorganisms by a new electrostatic precipitator. J. Aerosol Sci. 33, 1417-1432 (2002b)

C. Maneerat, Y. Hayata, N. Egashira, K. Sakamoto, Z. Hamai, M. Kuroyanagi, Photocatalytic reaction of $\mathrm{TiO}_{2}$ to decompose ethylene in fruit and vegetable storage. Trans. ASAE 46, 725-730 (2003)

J. Mankowski, M. Kristiansen, A review of short pulse generator technology. IEEE Trans. Plasma Sci. 28, 102-108 (2000)

P. Marracino, A. Paffi, R. Reale, M. Liberti, G. d'Inzeo, F. Apollonio, Technology of high-intensity electric-field pulses: a way to control protein unfolding. J. Phys. Chem. Biophys. 3, 1000117 (2013)

J.C. Martin, Nanosecond pulse techniques. Proc. IEEE 80, 934-945 (1992)

J.H. Martin, W.E. Leonanrd, Principles of field crop production (Macmillan, New York, 1967)

F. Masotti, S. Cattaneo, M. Stuknyt, I. De Noni, Airborne contamination in the food industry: an update on monitoring and disinfection techniques of air. Trends Food Sci. Technol. 90, 147-156 (2019)

J.W. Mastalerz, The Greenhouse Environment (Wiley, New York, 1977)

K. Matra, Non-thermal plasma for germination enhancement of radish seeds. Proc. Comput. Sci. 86, 132135 (2016)

G.A. Mesyats, Pulsed Power (Springer Science and Business Media, Berlin, 2007). https://doi.org/10. $1007 / \mathrm{b} 116932$

V. Mildaziene, G. Pauzaite, Z. Nauciené, A. Malakauskiene, R. Zukiene, I. Januskaitiene, V. Jakstas, L. Ivanauskas, I. Filatova, V. Lyushkevich, Pre-sowing seed treatment with cold plasma and electromagnetic field increases secondary metabolite content in purple coneflower (Echinacea purpurea) leaves. Plasma Process Polym. 15, e1700059 (2018)

S. Min, G.A. Evrendilek, H.Q. Zhang, Pulsed electric fields: processing system, microbial and enzyme inhibition, and shelf life extension of foods. IEEE Trans. Plasma Sci. 35, 59-73 (2007)

B.W. Mitchell, R.J. Buhr, M.E. Berrang, J.S. Bailey, N.A. Cox, M.E.T. Al, Reducing airborne pathogens, dust and salmonella transmission in experimental hatching cabinets using an electrostatic space charge system. Poult. Sci. 81, 49-55 (2002)

A. Mitra, Y.F. Li, T.G. Klämpfl, T. Shimizu, J.J. Gregor, E. Morfill, J.L. Zimmermann, Inactivation of surface-borne microorganisms and increased germination of seed specimen by cold atmospheric plasma. Food Bioprocess Technol. 7, 645-653 (2014)

F. Mitsugi, T. Abiru, T. Ikegami, K. Ebihara, S. Aoqui, K. Nagahama, Influence of ozone generated by surface barrier discharge on nematode and plant growth. IEEE Trans. Plasma Sci. 44, 3071-3076 (2016)

T. Miura, T. Sato, K. Arima, S. Mukaigawa, K. Takaki, T. Fujiwara, Duty factor effect on ozone production using dielectric barrier discharge reactor driven by IGBT pulse modulator. J. Adv. Oxid. Technol. 10, 311-315 (2007)

A. Mizuno, Electrostatic precipitation. IEEE Trans. Dieletr. Electr. Insul. 7, 615-624 (2000)

M.E.A. Mohamed, A.H.A. Eissa, "Pulsed Electric Fields for Food Processing Technology", Structure and Function of Food Engineering, Chap 11 (IntechOpen, London, 2012), p. 275 
J.-D. Moon, Acceleration of germination of tomato seed by applying AC electric and magnetic fields. J. Electrost. 481, 103-114 (2000)

R. Morar, R. Munteanu, E. Simion, I. Munteanu, L. Dascalescu, Electrostatic treatment of bean seeds. IEEE Trans. Ind. Appl. 35, 208-212 (1999)

C.L. Moretti, L.M. Mattos, A.G. Calbo, S.A. Sargent, Climate changes and potential impacts on postharvest quality of fruit and vegetable crops: a review. Food Res. Int. 43, 1824-1832 (2010)

L.E. Murr, Mechanism of plant-cell damage in an electrostatic field. Nature (lond.) 201, 1305-1306 (1964)

L.E. Murr, Biophysics of plant growth in an electrostatic field. Nature 206, 467-470 (1965)

L.E. Murr, The biophysics of plant growth in a reversed electrostatic field. A comparison with conventional electrostatic and electrokinetic field growth responses. Int. J. Biometeorol. 10, 135-146 (1966)

A. Nakagawa, H. Hatayama, K. Takaki, K. Koide, Y. Kawamura, Influence of pulse width on polyphenol extraction from agricultural products by pulsed electric field. IEEJ Trans. Fundam. Mater. 133, 32-37 (2013). (in Japanese)

Y. Nakagawa, R. Ono, T. Oda, Effect of discharge polarity on $\mathrm{OH}$ density and temperature in coaxialcylinder barrier discharge under atmospheric pressure humid air. Jpn. J. Appl. Phys. 57, 096103 (2018)

N. Nakamura, A. Fukushima, H. Iwayama, H. Suzuki, Electrotropism of pollen tubes of camellia and other plants. Sex. Plant Reprod. 4, 138-143 (1991)

T. Namihira, S. Tsukamoto, D. Wang, S. Katsuki, R. Hackam, H. Akiyama, Y. Uchida, M. Koike, Improvement of $\mathrm{NO}_{X}$ removal efficiency using short width pulsed power. IEEE Trans. Plasma Sci. 28, 434-442 (2000)

G.B. Ndiffo Yemeli, R. Švubová, D. Kostolani, S. Kyzek, Z. Machala, The effect of water activated by nonthermal air plasma on the growth of farm plants: case of maize and barley. Plasma Process Polym. 18, e2000205 (2020)

N. Nomura, M. Yano, S. Katsuki, H. Akiyama, K. Abe, S. Abe, Intracellular DNA damage induced by non-thermal, intense narrowband electric fields. IEEE Trans. Dielectr. Electr. Ins. 16, 1288-1293 (2007)

T. Nonomura, Y. Matsuda, L. Xu, K. Kakutani, Y. Takikawa, H. Toyoda, Collection of highly germinative pseudochain conidia of Oidium neolycopersici from conidiophores by electrostatic attraction. Mycol. Res. 113, 364-372 (2009)

R. Norarat, W. Thipprasert, C. Mikhamlueang, T. Wanmanee, N. Nanta, N. Srijumpa, Effects of high voltage stimulation and oxygen rich fine bubble (FB) water on cultivated shiitake mushroom in Thailand. Int. J. Plasma Environ. Sci. Technol. 12, 69-73 (2019)

J.O. Noyce, J.F. Hughes, Bactericidal effects of negative and positive ions generated in nitrogen on Escherichia coli. J. Electrostat. 54, 179-187 (2002)

S. Ohga, S. Iida, Effect of electric impulse on sporocarp formation of ectomycorrhizal fungus Laccaria laccata in Japanese red pine plantation. J. for. Res. 6, 37-41 (2001)

S. Ohga, S. Iida, C.-D. Koo, N.-S. Cho, Effect of electric impulse on fruit body production on Lentinula edodes in the sawdust-based substrate. Mushroom Sci. Biotechnol. 9, 7-12 (2001)

S. Ohga, N.-S. Cho, Y. Li, D.J. Royse, Utilization of pulsed power to stimulate fructification of edible mushroom. Mushroom Sci. 16, 343-352 (2004)

T. Ohshima, M. Sato, M. Saito, Selective release of intracellular protein using pulsed electric field. J. Electrostat. 35, 103-112 (1995)

T. Ohshima, K. Sato, H. Terauchi, M. Sato, Physical and chemical modifications of high-voltage pulse sterilization. J. Electrostat. 42, 159-166 (1997)

T. Ohshima, Y. Hama, M. Sato, Releasing profiles of gene products from recombinant Escherichia coli in a high-voltage pulsed electric field. Biochem. Eng. J. 5, 149-155 (2000)

T. Ohshima, T. Tamura, M. Sato, Influence of pulsed electric field on various enzyme activities. J. Electrost. 65, 156-161 (2007)

T. Ohshima, T. Tanino, T. Kameda, H. Harashima, Engineering of operation condition in milk pasteurization with PEF treatment. Food Control 68, 297-302 (2016)

T. Ohshima, T. Tanino, A. Guionet, K. Takahashi, K. Takaki, Mechanism of pulsed electric field enzyme activity change and pulsed discharge permeabilization of agricultural products. Jpn. J. Appl. Phys. 60, 060501 (2021)

T. Okumura, Y. Muramoto, N. Shimizu, Influence of DC electric field on growth of daikon radish. IEEE Trans. Dielectr. Electr. Insul. 19, 2237-2241 (2012) 
T. Okumura, Y. Saito, K. Takano, K. Takahashi, K. Takaki, N. Satta, T. Fujio, Inactivation of bacteria using discharge plasma under liquid fertilizer in a hydroponic culture system. Plasma Med. 6, 247-254 (2016)

R. Ono, S. Uchida, N. Hayashi, R. Kosaka, Y. Soeda, Inactivation of bacteria on plant seed surface by low-pressure RF plasma using a vibrating stirring device. Vacuum 136, 214-220 (2017)

D.I. Onwude, G. Chen, N. Eke-emezie, A. Kabutey, A.Y. Khaled, B. Sturm, Recent advances in reducing food losses in the supply chain of fresh agricultural produce. Processes 8, 1431 (2020)

L.G. Paleg, D. Aspinall, J. Gen. Physiol. 15, 391-420 (1932)

D.P. Park, K. Davis, S. Gilani, C.A. Alonzo, D. Dobrynin, G. Friedman, A. Fridman, A. Rabinovich, G. Fridman, Reactive nitrogen species produced in water by non-equilibrium plasma increase plant growth rate and nutritional yield. Curr. Appl. Phys. 13, S19-29 (2013)

Y. Park, K.S. Oh, J. Oh, D.C. Seok, S.B. Kim, S.J. Yoo, M.J. Lee, The biological effects of surface dielectric barrier discharge on seed germination and plant growth with barley. Plasma Process. Polym. 15, 1600056 (2016)

G. Pataro, M. De Lisi, G. Donsi, G. Ferrari, Microbial inactivation of E-coli cells by a combined PEFHPCD treatment in a continuous flow system. Innov. Food Sci. Emerg. Technol. 22, 102-109 (2013)

S.M. Perez, E. Biondi, R. Laurita, M. Proto, F. Sarti, M. Gherardi, A. Bertaccini, V. Colombo, Plasma activated water as resistance inducer against bacterial leaf spot of tomato. PLoS ONE 14, e0217788 (2019)

R. Pratt, Effect of ionized air on early growth of black mustard seedlings. J. Pharm. Sci. 51, 184-185 (1962)

N. Puač, M. Gherardi, M. Shiratani, Plasma agriculture: a rapidly emerging field. Plasma Process Polym. 15, e1700174 (2018)

Z. Qin, M.J. Buehler, Molecular dynamics simulation of the a-helix to b-sheet transition in coiled protein filaments: evidence for a critical filament length scale. Phys. Rev. Lett. 104, 198304 (2010)

L.K. Randeniya, G.J.J.B. Groot, Non-thermal plasma treatment of agricultural seeds for stimulation of germination, removal of surface contamination and other benefits: a review. Plasma Process. Polym. 12, 608-623 (2015)

P. Ranieri, N. Sponsel, J. Kizer, M.R. Pierce, R. Hernández, L. Gatiboni, A. Grunden, K. Stapelmann, Plasma agriculture: review from the perspective of the plant and its ecosystem. Plasma Process Polym. 18, e2000162 (2020)

K.S. Rathore, A. Goldsworthy, Electrical control of shoot regeneration in plant tissue cultures. Bio. Technol. 3, 1107-1109 (1985)

S. Reuter, T. von Woedtke, K.-D. Weltmann, The kINPen - a review on physics and chemistry of the atmospheric pressure plasma jet and its applications. J. Phys. D Appl. Phys. 51, 233001 (2018)

W. Ross, Galvanic experiments on vegetation. US Patent Office Report, pp. 370-373 (1844)

S.N. Rukin, High-power nanosecond pulse generators based on semiconductor opening switches (review). Instrum. Exp. Tech. 42, 439-467 (1999)

H.M. Rustebakke, Electric utility systems and practices (Wiley, New York, 1983)

Y. Sakamoto, Influences of environmental factors on fruiting body induction, development and maturation in mushroom-forming fungi. Fungal Biol. Rev. 32, 236-248 (2018)

J.W. Sanborn, Electroculture of plants. Utah Agricultural Experiment Station Report, pp. 71-74 (1893)

R. Sánchez-Vega, P. Elez-Martínez, O. Martín-Belloso, Influence of high-intensity pulsed electric field processing parameters on antioxidant compounds of broccoli juice. Innov. Food Sci. Emerg. Technol. 29, 70-77 (2014)

T. Sarinont, T. Amano, P. Attri, K. Koga, N. Hayashi, M. Shiratani, Effects of plasma irradiation using various feeding gases on growth of Raphanus sativus L. Arch. Biochem. Biophys. 605, 129-140 (2016)

M. Sato, N.M. Ishida, A.T. Sugiarto, T. Ohshima, H. Taniguchi, High-efficiency sterilizer by high-voltage pulse using concentrated-field electrode system. IEEE Trans. Ind. Appl. 37, 1646-1650 (2001)

K.H. Schoenbach, F.E. Peterkin, R.W. Alden III., S.J. Beebe, The effect of pulsed electric fields on biological cells: experiments and applications. IEEE Trans. Plasma Sci. 25, 284-292 (1997)

K.H. Schoenbach, B. Hargrave, R.P. Joshi, J.F. Kolb, R. Nuccitelli, C. Osgood, A. Pakhomov, M. Stacey, R.J. Swanson, J.A. White, S. Xiao, J. Zhang, S.J. Beebe, P.F. Blackmore, E.S. Buescher, Bioelectric effects of intense nanosecond pulses. IEEE Trans. Dielectr. Electr. Ins. 14, 1088-1109 (2007)

K.H. Schoenbach, S. Xiao, R.P. Joshi, J.T. Camp, T. Heeren, J.F. Kolb, S.J. Beebe, The effect of intense subnanosecond electrical pulses on biological cells. IEEE Trans. Plasma Sci. 36, 414-422 (2008) 
A.R. Schrank, Bio electric fields and growth (University of Texas Press, 1947)

B. Sera, M. Sery, Non-thermal plasma treatment as a new biotechnology in relation to seeds, dry fruits, and grains. Plasma Sci. Technol. 20, 044012 (2018)

B. Sera, I. Gajdova, M. Sery, P. Spatenka, New physicochemical treatment method of poppy seeds for agriculture and food industries. Plasma Sci. Technol. 15, 935-938 (2013)

B. Sera, M. Sery, B. Gavril, I. Gajdova, Seed germination and early growth responses to seed pretreatment by non-thermal plasma in hemp cultivars (Cannabis sativa L.). Plasma Chem. Plasma Process. 37, 207-221 (2017)

K. Shamsi, C. Versteeg, F. Sherkat, J. Wan, Alkaline phosphatase and microbial inactivation by pulsed electric field in bovine milk. Innov. Food Sci. Emerg. Technol. 9, 217-223 (2008)

P. Sharma, P. Bremer, I. Oey, D.W. Everett, Bacterial inactivation in whole milk using pulsed electric field processing. Int. Dairy J. 35, 49-56 (2014a)

P. Sharma, I. Oey, P. Bremer, D.W. Everett, Reduction of bacterial counts and inactivation of enzymes in bovine whole milk using pulsed electric fields. Int. Dairy J. 39, 146-156 (2014b)

S.-Y. Sheu, D.-Y. Yang, H.L. Selzle, E.W. Schlag, Energetics of hydrogen bonds in peptides. Proc. Natl Acad. Sci. USA 100, 12683-12678 (2003)

M. Shibusawa, K. Shibata, The effect of electrical discharges on the rate of growth of plants. J. Inst. Electr. Eng. Jpn. 47, 1259-1300 (1927)

T. Shigemitsu, Effects of electric fields, air ion and corona discharge on plants. J. Plasma Fusion Res. 75, 659-665 (1999)

S. Shiina, T. Ohshima, M. Sato, Extracellular release of recombinant $\alpha$-amylase from Escherichia coli using pulsed electric field. Biotechnol. Prog. 20, 1528-1533 (2004)

S. Shiina, T. Ohshima, M. Sato, Extracellular production of $\alpha$-amylase during fed-batch cultivation of recombinant Escherichia coli using pulsed electric field. J. Electrost. 65, 30-36 (2007)

H. Shimizu, T. Hiraguri, M. Kimoto, K. Ota, T. Shindo, Y. Hoshino, K. Takaki, Stimulatory growth effect of lightning strikes applied in the vicinity of shiitake mushroom bed logs. J. Phys. D Appl. Phys. 53, 204002 (2020)

L.W. Sieck, J.T. Herron, D.S. Green, Chemical kinetics database and predictive schemes for humid air plasma chemistry. Part I: positive ion-molecule reactions. Plasma Chem. Plasma Process. 20, 235257 (2000)

C. Siemer, S. Toepfl, V. Heinz, Inactivation of Bacillus subtilis spores by pulsed electric fields (PEF) in combination with thermal energy-I. Influence of processand product parameters. Food Control 39, 163-171 (2014)

F.M. Silva, K.V. Chau, J.K. Brecht, S.A. Sargent, Modified atmosphere packaging for mixed loads of horticultural commodities exposed to two postharvest temperatures. Postharvest Biol. Technol. 17, $1-9$ (1999)

L. Sivachandiran, A. Khacef, Enhanced seed germination and plant growth by atmospheric pressure cold air plasma: combined effect of seed and water treatment. RSC Adv. 7, 1822-1832 (2017)

L.J. Skog, C.L. Chu, Effect of ozone on qualities of fruits and vegetables in cold storage. Can. J. Plant Sci. 81, 773-778 (2001)

P.W. Smith, Transient Electronics: Pulsed Circuit Technology (Wiley, New York, 2002)

E. Solly, The influence of electricity on vegetation. J. Horticult. Soc. 1, 81-109 (1845)

J.-S. Song, S.B. Kim, S. Ryu, J. Oh, D.-S. Kim, Emerging plasma technology that alleviates crop stress during the early growth stages of plants: a review. Front. Plant Sci. 11, 988 (2020)

T. Sonoda, N. Takamura, D. Wang, T. Namihira, H. Akiyama, Growth control of leaf lettuce using pulsed electric field. IEEE Trans. Plasma Sci. 42, 3202-3208 (2014)

T. Sonoda, Y. Higashi, Y. Yamada, D. Wang, T. Namihira, H. Akiyama, Influence of pulsed electric field to leaf lettuce evaluated on chlorophyll fluorescence measurement using pulsed-amplitude-modulated fluorometer. Int. J. Plasma Environ. Sci. Technol. 11, 81-86 (2017)

N.V. Statsyuk, K. Thakur, T.I. Smetanina, M.A. Kuznetsova, Effect of a pre-planting treatment of seed tubers with low-frequency pulse electric field on the growth of potato plants of different varieties. Agric. Biol. 51, 360-366 (2016)

E. Stoffels, I.E. Kieft, R.E.J. Sladek, L.J.M. van den Bedem, E.P. van der Laan, M. Steinbuch, Plasma needle for in vivo medical treatment: recent developments and perspectives. Plasma Sources Sci. Technol. 15, S169-S180 (2006)

T. Stolárik, M. Henselová, M. Martinka, O. Novák, A. Zahoranová, M. Černák, Effect of low-temperature plasma on the structure of seeds, growth and metabolism of endogenous phytohormones in pea (Pisum sativum L.). Plasma Chem. Plasma Process. 35, 659-676 (2015) 
G.E. Stone, The influence of current electricity on plant growth. Hatch Experiment Station (Mass.). Annu. Rep. 16, 13-30 (1904)

G.E. Stone, Influence of electricity on microorganisms. Bot. Gaz. 48, 359-379 (1909)

R. Straessner, C. Eing, M. Goettel, C. Gusbeth, W. Frey, Monitoring of pulsed electric field-induced abiotic stress on microalgae by chlorophyll fluorescence diagnostic. IEEE Trans. Plasma Sci. 41, 2951-2958 (2013)

B. Su, J. Guo, W. Nian, H. Feng, K. Wang, J. Zhang, J. Fang, Early growth effects of nanosecond pulsed electric field (nsPEFs) exposure on Haloxylon ammodendron. Plasma Process. Polym. 12, 372-379 (2015)

A. Sudrajad, A. Fitri, Review of electrostatic precipitator device for reduce of diesel engine particulate matter. Energy Procedia 68, 370-380 (2015)

P. Sun, Y. Sun, H. Wu, W. Zhu, J.L. Lopez, W. Liu, J. Zhan, R. Li, J. Fang, Atmospheric pressure cold plasma as an antifungal therapy. Appl. Phys. Lett. 98, 021501 (2011)

Q.A. Syed, A. Ishaq, U.U. Rahman, S. Aslam, R. Shukat, Pulsed electric field technology in food preservation: a review. J. Nutr. Health Food Eng. 6, 168-172 (2017)

K. Takahashi, Chapter 15: Keeping freshness of agricultural products, in Agritech: Innovative Agriculture Using Microwaves and Plasmas. ed. by S. Horikoshi, G. Brodie, K. Takaki, N. Serpone (Springer, 2021). (in press)

K. Takahashi, Y. Sasaki, S. Mukaigawa, K. Takaki, T. Fujiwara, N. Satta, Purification of high-conductivity water using gas-liquid phase discharge reactor. IEEE Trans. Plasma Sci. 38, 2694-2700 (2010)

K. Takahashi, I. Yagi, K. Takaki, N. Satta, Development of pulsed discharge inside bubble in water. IEEE Trans. Plasma Sci. 39, 2654-2655 (2011)

K. Takahashi, K. Takaki, N. Satta, Water remediation using pulsed power discharge under water with an advanced oxidation process. J. Adv. Oxid. Technol. 15, 365-373 (2012)

K. Takahashi, K. Miyamoto, K. Takaki, K. Takahashi, Development of compact high-voltage power supply for stimulation to promote fruiting body formation in mushroom cultivation. Materials 11, 2471 (2018)

K. Takahashi, S. Kawamura, I. Yagi, M. Akiyama, K. Takaki, N. Satta, Influence of reactor geometry and electric parameters on wastewater treatment using discharge inside a bubble. Int. J. Plasma Environ. Sci. Technol. 13, 74-82 (2019a)

K. Takahashi, K. Takaki, I. Hiyoshi, Y. Enomoto, S. Yamaguchi and H. Nagata, Modern applications of electrostatics and dielectrics; development of a corona discharge ionizer utilizing high-voltage AC power supply driven by PWM inverter for highly efficient electrostatic elimination. IntechOpen Ltd., London, ISBN 978-1-78984-557-0 (2019b)

K. Takahashi, R. Saito, T. Onodera, K. Takaki, H. Kitai, K. Sakamoto, Development of compact inductive energy storage pulsed-power generator driven by $13 \mathrm{kV}$ SiC-MOSFET. Rev. Sci. Instrum. 92, 064706 (2021)

J. Takahata, K. Takaki, N. Satta, K. Takahashi, T. Fujio, Y. Sasaki, Improvement of growth rate of plants by bubble discharge in water. Jpn. J. Appl. Phys. 54, 01AG07 (2015)

K. Takaki, Chapter 14: Promotion of reproductive growth of mushroom using electrical stimuli, in Agritech: Innovative Agriculture Using Microwaves and Plasmas. ed. by S. Horikoshi, G. Brodie, K. Takaki, N. Serpone (Springer, 2021). (in press)

K. Takaki, K. Kanesawa, N. Yamazaki, S. Mukaigawa, T. Fujiwara, K. Takahasi, K. Yamasita, K. Nagane, Effect of pulsed high-voltage stimulation on Pholiota nameko mushroom yield. Acta Phys. Pol. A 115, 953-956 (2004)

K. Takaki, K. Kanesawa, S. Mukaigawa, T. Fujiwara, Energy efficiency of corona discharge reactor driven by inductive energy storage system pulsed power generator. I. IEEE Trans. Dielectr. Electr. Insul. 14, 834-845 (2007)

K. Takaki, K. Kanesawa, N. Yamazaki, S. Mukaigawa, T. Fujiwara, K. Takahasi, K. Yamasita, K. Nagane, Improvement of edible mushroom yield by electric stimulations. J. Plasma Fusion Res. Ser. 8, 556559 (2009)

K. Takaki, K. Takahashi, T. Ueno, M. Akiyama, T. Sakugawa, Design and practice of pulsed power circuit. J. Plasma Fusion Res. 87, 202-215 (2011)

K. Takaki, J. Takahata, S. Watanabe, N. Satta, O. Yamada, T. Fujio, Y. Sasaki, Improvements in plant growth rate using underwater discharge. J. Phys. Conf. Ser. 418, 012140 (2013)

K. Takaki, K. Yoshida, T. Saito, T. Kusaka, R. Yamaguchi, K. Takahashi, Y. Sakamoto, Effect of electrical stimulation on fruit body formation in cultivating mushrooms. Microorganisms 2, 58-72 (2014) 
K. Takaki, J. Nishimura, S. Koide, K. Takahashi, T. Uchino, Decomposition of ethylene using dual-polarity pulsed dielectric barrier discharge. IEEE Trans. Plasma Sci. 43, 3476-3482 (2015)

K. Takaki, K. Takahashi, S. Sakamoto, Chapter 7: High-voltage methods for mushroom fruit-body developments, in Plant and Mushroom Development. ed. by M.A. El-Esaw (Intech Open Ltd., London, 2018), pp. 95-113

K. Takaki, N. Hayashi, D. Wang, T. Ohshima, High-voltage technologies for agriculture and food processing. J. Phys. D Appl. Phys. 52, 473001 (2019)

K. Takaki, K. Takahashi, D. Hamanaka, R. Yoshida, T. Uchino, Function of plasma and electrostatics for keeping quality of agricultural produce in post-harvest stage. Jpn. J. Appl. Phys. 60, 010501 (2020)

N. Takeuchi, K. Yasuoka, Review of plasma-based water treatment technologies for the decomposition of persistent organic compounds. Jpn. J. Appl. Phys. 60, SA0801 (2021)

K. Tanaka, Plasma thermal and non-thermal technologies, in Agritech: Innovative Agriculture Using Microwaves and Plasmas Thermal and Non-Thermal Processing. ed. by S. Horikoshi, G. Brodie, K. Takaki, N. Serpone (Springer, Tokyo, 2021)

C. Tendero, C. Tixier, P. Tristant, J. Desmaison, P. Leprince, Atmospheric pressure plasmas: a review. Spectrochim. Acta B 61, 2-30 (2006)

Y. Teramoto, D. Deguchi, I.V. Lisitsyn, T. Namihira, T. Katsuki, H. Akiyama, All-solid-state triggerless repetitive pulsed power generator utilizing a semiconductor opening switch. Rev. Sci. Instrum. 72, 4464-4468 (2001)

M.P. Thakur, Advances in mushroom production: key to food, nutritional and employment security: a review. Indian Phytopathol. 73, 377-395 (2020)

J. Tong, R. He, X. Zhang, R. Zhan, W. Chen, S. Yang, Plasma pretreatment on the seed germination and early growth of Andrographis paniculate. Plasma Sci. Technol. 16, 260-266 (2014)

M.A. Torres, ROS in biotic interactions. Physiol. Plant. 138, 414-429 (2010)

Y. Toyokawa, Y. Yagyu, T. Misawa, A. Sakudo, A new roller conveyer system of non-thermal gas plasma as a potential control measure of plant pathogenic bacteria in primary food production. Food Control 72, 62-72 (2017)

E.D. Valle, P. Marracino, O. Pakhomova, M. Liberti, F. Apollonio, Nanosecond pulsed electric signals can affect electrostatic environment of proteins below the threshold of conformational effects: the case study of SOD1 with a molecular simulation study. PLoS ONE 14, e0221685 (2019)

N.D. Vaze, M.J. Gallagher, S. Park, G. Fridman, V.N. Vasilets, A.F. Gutsol, S. Anandan, G. Fridman, A.A. Fridman, Inactivation of bacteria in flight by direct exposure to nonthermal plasma. IEEE Trans. Plasma Sci. 37, 3234-3240 (2010)

N.D. Vaze, S. Park, A.D. Brooks, A. Fridman, G. Joshi, Involvement of multiple stressors induced by nonthermal plasma-charged aerosols during inactivation of airborne bacteria. PLoS ONE 12, e0171434 (2017)

H.M. Vega-Mercado, O. Martin-Belloso, B.L. Qin, F.J. Chang, M.M. Góngora-Nieto, G.V. Barbara-Cánovas, B.G. Swanson, Nonthermal food preservation: pulsed electric fields. Trends Food Sci. Technol. 8, $151(1997)$

C.C.W. Verlackt, W. Van Boxem, A. Bogaerts, Transport and accumulation of plasma generated species in aqueous solution. Phys. Chem. Chem. Phys. 20, 6845-6859 (2018)

E. Vorobiev, N.I. Lebovka, Electrotechnologies for extraction from food plants and biomaterials (Springer, New York, 2008)

D. Wang, Chapter 13: Improvement of plant growth and control of cultivation environment using electrical stimuli, in Agritech: Innovative Agriculture Using Microwaves and Plasmas. ed. by S. Horikoshi, G. Brodie, K. Takaki, N. Serpone (Springer, 2021), p. in press

Y. Wang, B. Wang, L. Li, Keeping quality of tomato fruit by high electrostatic field pretreatment during storage. J. Sci. Food Agric. 88, 464-470 (2008)

G. Wang, J. Huang, W. Gao, J. Lu, J. Li, R. Liao, C.A. Jaleel, The effect of high-voltage electrostatic field (HVEF) on aged rice (Oryza sativa L.) seeds vigor and lipid peroxidation of seedlings. J. Electrost. 67, 759-764 (2009)

P. Wang, F. Fan, F. Zirilli, J. Chen, A hybrid model to predict electron and ion distributions in entire interelectrode space of a negative corona discharge. IEEE Trans. Plasma Sci. 40, 421-428 (2012)

K. Weltmann, E. Kindel, T. von Woedtke, Atmospheric pressure plasma sources: prospective tools for plasma medicine. Pure Appl. Chem. 82, 1223-1237 (2010) 
I.D. Wilson, S.L. Neill, J.T. Hancock, Nitric oxide synthesis and signalling in plants. Plant Cell Environ. 31, 622-631 (2008)

T. Woedtke, S. Reuter, K. Masur, K.D. Weltmann, Plasmas for medicine. Phys. Rep. 530, 291-320 (2013)

E. Wollny, Elektrische Kulturversuche. Forsch. Agr. Phys. 11, 88-112 (1893)

C. Wolverton, J.L. Mullen, H. Ishikawa, M.L. Evans, Two distinct regions of response drive differential growth in Vigna root electrotropism. Plant Cell Environ. 23, 1275-1280 (2000)

S. Yamazaki, S. Tanaka, H. Tsukamoto, Kinetic studies of oxidation of ethylene over a $\mathrm{TiO}_{2}$ photocatalyst. J. Photochem. Photobiol. A 121, 55-61 (1999)

N. Yang, K. Huang, C. Lyu, J. Wang, Pulsed electric field technology in the manufacturing processes of wine, beer, and rice wine: a review. Food Control 61, 28-38 (2016)

H. Ye, L.L. Huang, S.D. Chen, J.J. Zhong, Pulsed electric field stimulates plant secondary metabolism in suspension cultures of Taxus chinensis. Biotechnol. Bioeng. 88, 788-795 (2004)

H.W. Yeom, Q.H. Zhang, Enzymatic inactivation by pulsed electric fields: a review, in Pulsed Electric Fields in Food Processing. ed. by G.V. Barbosa-Cánovas, Q.H. Zhang (Technomic Publishing, Lancaster, 2001), pp. 57-63

H.W. Yeom, C.B. Streaker, Q.H. Zhang, D.B. Min, Effects of pulsed electric fields on the activities of microorganisms and pectin methyl esterase in orange juice. J. Food Sci. 65, 1359-1363 (2000)

J.Y. Yi, J.W. Choi, B.Y. Jeon, I.L. Jung, D.H. Park, Effects of a low-voltage electric pulse charged to culture soil on plant growth and variations of the bacterial community. Agric. Sci. 3, 339-346 (2012)

M. Yin, M. Huang, B. Ma, T. Ma, Stimulating effects of seed treatment by magnetized plasma on tomato growth and yield. Plasma Sci. Technol. 7, 3143-3147 (2005)

A. Zaderej, C.E. Corson, Electrogenic seed treater US 4302670 (1970)

H. Zhang, F. Hashinaga, Effect of high electric fields on the germination and early growth of some vegetable seeds. J. Jpn. Soc. Hortic. Sci. 66, 347-352 (1997)

S. Zhang, A. Rousseau, T. Dufour, Promoting lentil germination and stem growth by plasma activated tap water, demineralized water and liquid fertilizer. RSC Adv. 7, 31244-31251 (2017)

W. Zhao, R. Yang, X. Shen, S. Zhang, X. Chen, Lethal and sublethal injury and kinetics of Escherichia coli, Listeria monocytogenes and Staphylococcus aureus in milk by pulsed electric fields. Food Control 32, 6-12 (2013)

Z. Zhou, Y. Huang, S. Yang, W. Chen, Introduction of a new atmospheric pressure plasma device and application on tomato seeds. Agric. Sci. 2, 23-27 (2011)

P. Zhou, Y. Yang, A.C.K. Lai, G. Huang, Inactivation of airborne bacteria by cold plasma in air duct flow. Build. Environ. 106, 120-130 (2016)

U. Zimmermann, Electric field-mediated fusion and related electrical phenomena. Biochem. Biophys. Acta 64, 227-277 (1982)

U. Zimmermann, G. Pilwat, F. Riemann, Dielectric breakdown of cell membranes. Biophys. J. 14, 881-899 (1974)

U. Zimmermann, G. Pilwat, F. Riemann, Preparation of erythrocyte ghosts by dielectric breakdown of the cell membrane. Biochim. Biophys. Acta 375, 209-219 (1975)

U. Zimmermann, G. Pilwat, F. Beckers, F. Riemann, Effects of external electrical fields on cell membranes. Bioelectrochem. Bioenerg. 3, 58-83 (1976)

R. Zukiene, Z. Nauciene, I. Januskaitiene, G. Pauzaite, V. Mildaziene, K. Koga, M. Shiratani, Dielectric barrier discharge plasma treatment-induced changes in sunflower seed germination, phytohormone balance, and seedling growth. Appl. Phys. Express 12, 126003 (2019)

R. Zvitov, A. Schwartz, E. Zamski, A. Nussinovitch, Direct current electrical field effects on intact plant organs. Biotechnol Prog. 19, 965-971 (2003)

Publisher's Note Springer Nature remains neutral with regard to jurisdictional claims in published maps and institutional affiliations. 


\section{Authors and Affiliations}

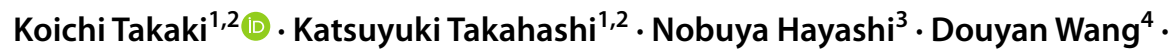
Takayuki Ohshima ${ }^{5}$

$\triangle$ Koichi Takaki

takaki@iwate-u.ac.jp

1 Faculty of Science and Engineering, Iwate University, Morioka, Iwate 020-8551, Japan

2 Agri-Innovation Center, Iwate University, Morioka, Iwate 020-8550, Japan

3 Department of Engineering Science, Kyusyu University, Kasuga, Fukuoka 816-8580, Japan

4 Institute of Industrial Nanomaterials, Kumamoto University, Chuo-ku, Kumamoto 860-8555, Japan

5 Faculty of Science and Technology, Gunma University, Kiryu, Gunma 376-8515, Japan 